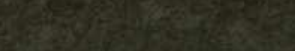




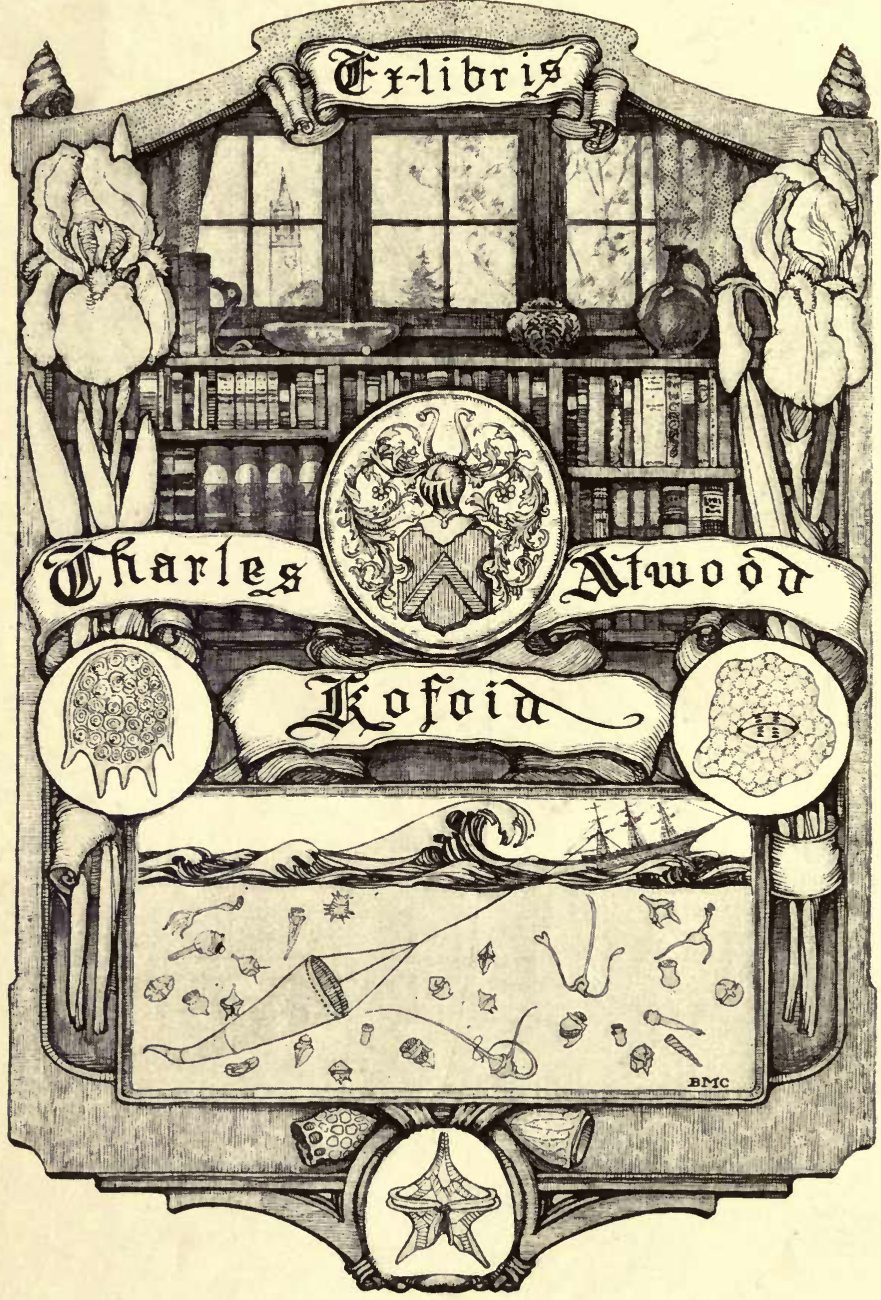




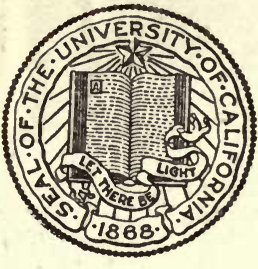

THE LIBRARY

OF

THE UNIVERSITY OF CALIFORNIA

\author{
PRESENTED BY
}

PROF. CHARLES A. KOFOID AND MRS. PRUDENCE W. KOFOID 



\title{
EXPERIMENTAL INQUIRY
}

\author{
INTO THE LAWS OF
}

ORGANIC AND ANIMAL LIFE. 


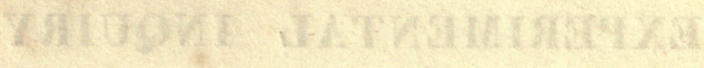

\section{.7T\%. WAMHA GKA . OHKADSTO}

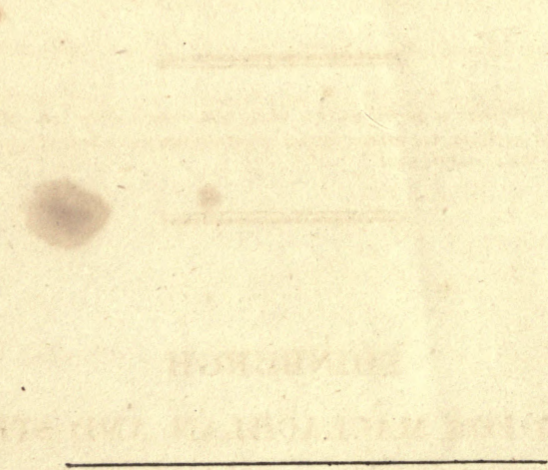

J. Moir, Printer, West Register street, Edinburgh. 


\title{
EXPERIMENTAL INQUIRY
}

\author{
INTO THE
}

LAWS WHICH REGULATE THE PHENOMENA

OF

\section{ORGANIC AND ANIMAL LIFE.}

BY

\section{GEORGE CALVERT HOLLAND, M. D.}

BACIELOR OF LETTERS OF THE UNIVERSITY OF PARIS ; FORMERLY SENIOR PRESIDENT OF TILE HUNTERIAV MEDICAL SOCIETY, AND PRESIDENT OF THE ROYAC PHYSIC, . SOCIETY

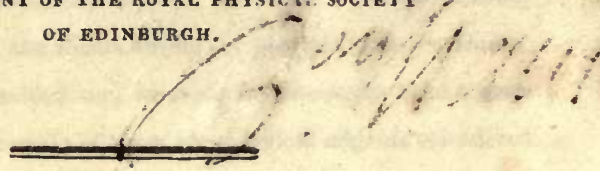

The application of particulars to a whole will onen discover their fallacy, and the applying any general system to particulars will often discover its true limits. CULLEN.

\section{EDINBURGH :}

PRINTED FOR MACLACHLAN AND STEWART; AND SIMPKIN AND MARSHALI, LONDON.

1829. 


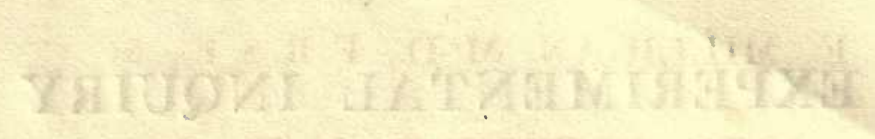

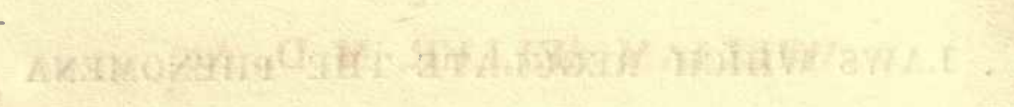

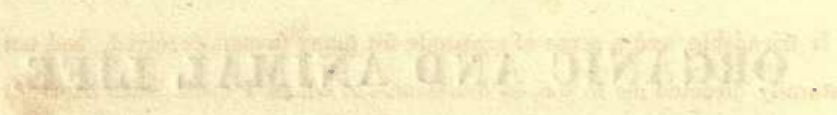

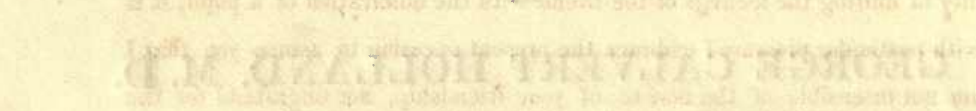

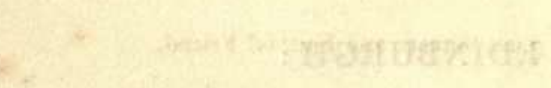

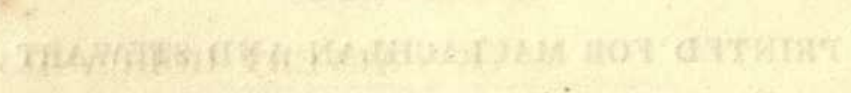




\title{
E. MILLIGAN, M. D., F.R.S.E., \&c. L. b,
}

\section{JOHN FLETCHER, M. D., \&c.}

\author{
AND \\ WILLIAM SELLER, M. D, \&c.
}

\section{MY Dear SIrs,}

Ir friendship, and a sense of gratitude for many favours received, had not naturally directed me to you, as individuals to whom I could, with heartfelt satisfaction, dedicate the present production, your well known intellectual acuteness and professional acquirements would alone have been sufficient to inspire a wish to possess the honour of your names. In having the opportunity of uniting the feelings of the friend with the admiration of a pupil, it is with particular pleasure I cmbrace the present occasion to assure you that I um not insensible of the honour of your friendship, nor ungrateful for the benefits you have conferred, and the interest you have shown in promoting the completion of this Physiological Inquiry. To you I am particularly indebted for having examined the sheets during the progress of their printing. Your liberal remarks have enabled me, in many instances, to take a more extended or correct view of certain of those physiological subjects considered in their ontline or detail in the present volume; and your approbation and encouragement have made me cultivate with pleasure what otherwise might have been a labour. Believe me,

\section{Mx Dear Sirs,}

Yoar Sincere and Faithful Friend,

\section{G. CALVER'T HOLLAND.}

MaNehesteh, Junc 2, 1829. 


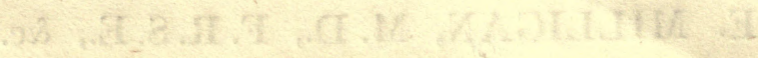

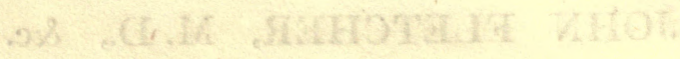

$$
\text { Whis: }
$$

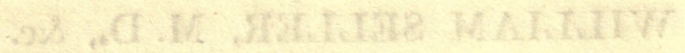

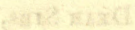

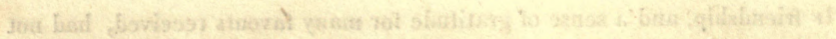

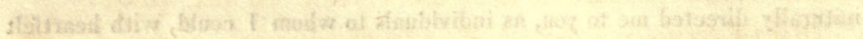

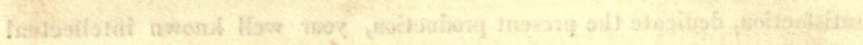

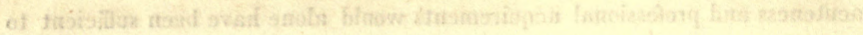

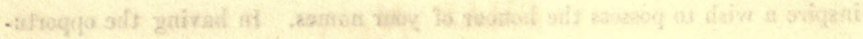

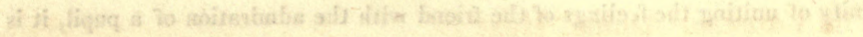

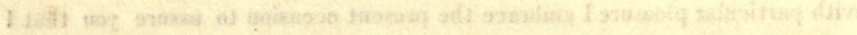

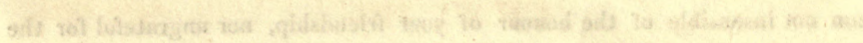

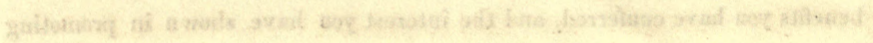

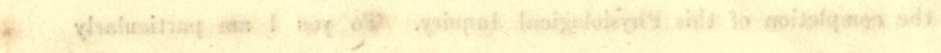

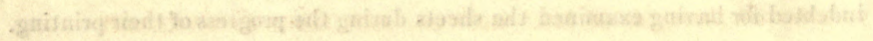

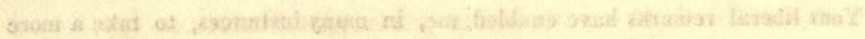

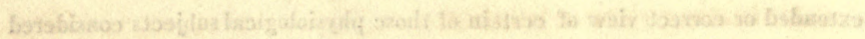

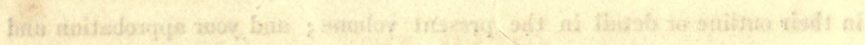

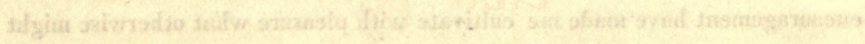

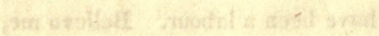

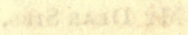

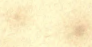

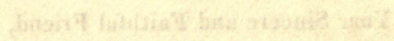

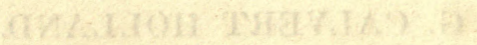




\section{CONTENTS.}

CHAP. I.

Page,

Animal Heat is not in the direct ratio of the quantity of oxygen inhaled, but in the inverse ratio of the quantity of blood exposed to this principle.

CHAP. II.

'I'he Cause of Animal Heat

\section{CHAP. III.}

Secreting surfaces are not dependent on Nervous communication between their own individual parts and the origin of the nerves, nor does Galvanism re-establish or excite their particular functions by maintaining such a relation ..... 68

\section{CHAP. IY.}

The Distribution of the Blood at different Ages and Seasons . . . . . . . . : 98

CHAP. V.

Temperature at different Ages

\section{CHAP. VI.}

The manner in which the System is adapted to the influence of Cold . . . . . . . 143 
CHAP. VII.

Page.

On the Torpidity of Hibernating Animals $\quad . \quad$. 161

\section{CHAP. VIII.}

The means by which the System is enabled to bear

a temperature much superior to that of the body 174

$$
\text { CHAP. IX. }
$$

The Influence of Disease on the production of Heat 189

$$
\text { CHAP. X. }
$$

The Function of the Eighth Pair of Nerves . . . 208

\section{CHAP. XI.}

The Influence of Narcotics on the generation of Animal Heat and the Digestive Powers . . . 241

\section{CHAP. XII.}

The Causes which influence the Action of the Heart 260

$$
\text { CHAP. XIII. }
$$

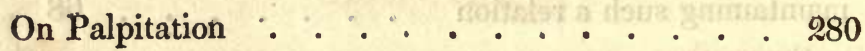

SEct. I.-The general causes which produce this affection . . . . . . . . ib. SEcT. II.-Organic causes which produce pal-

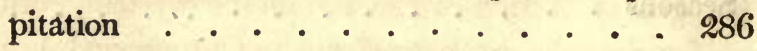

CHAP. XIV.

Syncope : . . . . . . . . . 300

SEcr. I.-The manner in which the Sanguiferous and Nervous Systems are acted upon by those causes which produce Syncope . . . ib. 
SECT. II.-An examination of the causes which occasion Syncope by changing the ordinary course or freedom of the circulation . . 316

CHAP. XV.

Physiology of Passion . . . . . . . . 324

Sect. I.-The Seat of Passion . . . . ib.

Secr. II.-The Phenomena and Physiology of Passion . . . . . . . . . 333

SEct. III.-The Influence and Effects of Passion . . . . . . . . . 350

\section{CHAP. XVI.}

An Inquiry into the Nature of the Vital Principle - 363

SEct. I.-An Investigation of the different phenomena attributed to an unknown or immaterial principle . . . . . . . . ib.

SEcr. II.-An Inquiry into the nature of that principle by means of which the System is maintained in health and vigour . . . 381

CHAP. XVII.

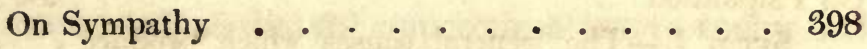

\section{CHAP. XVIII.}

The general Action of Emetics on the System, with a few remarks on their efficacy in Chronic and Acute Diseases . . . . . . . . . . 423 APPENDIX..........., 465 
oginat

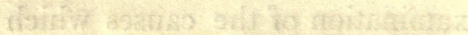

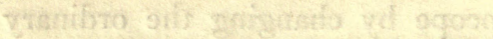

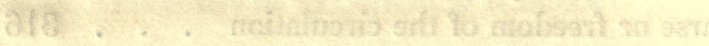

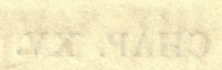

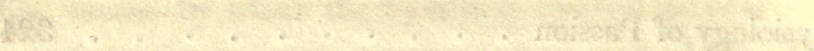

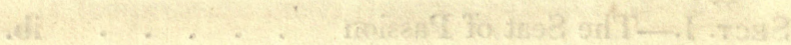

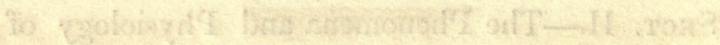

$7 x^{2}$

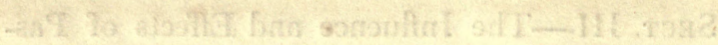
naso

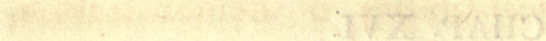

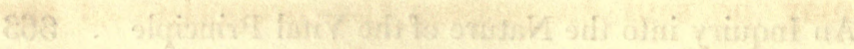

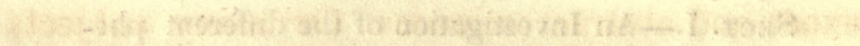

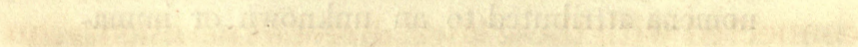
dit

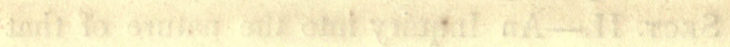

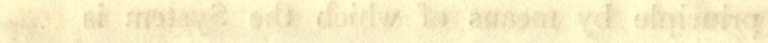

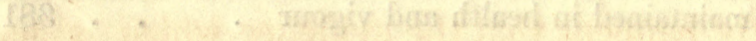

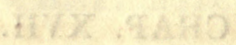

809

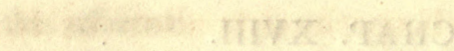

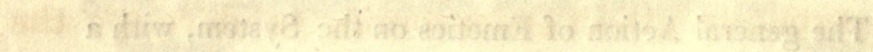

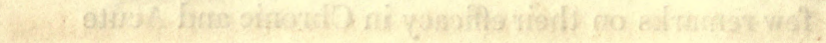
200

nes? nampaicit 2xpogemorit?

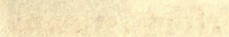




\section{INTRODUCTION.}

The Laws of Organic and Animal Life, in regard to their susceptibility of demonstration, may be said to possess the properties of the exact and abstract sciences. On many subjects connected with these laws, direct experiments can be brought forward to prove the correctness or fallacy of what is advanced ; but it likewise frequently happens that the evidence which is acquired, even in this way, must be considered to make only a distant approximation to many important truths. Experiments allow us to go a certain length in endeavouring to develope the different functions of the system; but unless the reflecting powers enable us to surpass the limits prescribed to our imperfect means and senses, many principles of importance, which are legitimately deduced from general 
facts and phenomena, would be too unconnected in their relations to serve any practical or useful purpose. When the animal system is patiently investigated, by means of well-directed experiments and deliberate observations, many mysteries are cleared up, and many intricacies are solved. When the laws of the constitution are thus examined, the results are similar to those that Dugald Stewart has described in respect to the operations of the mind, when studied with care: "Upon a slight attention to the operations of our own minds, they appear to be so complicated, and so infinitely diversified, that it seems to be impossible to reduce them to any general laws. In consequence, however, of a more accurate examination, the prospect clears up; and the phenomena, which appeared at first to be too various for our comprehension, are found to be the result of a comparatively small number of simple and uncompounded faculties, or of simple uncompounded principles."

We must guard against being too indulgent either to the experimentalist or theorist. The results obtained by the former require a more critical investigation than they generally receive,- 
not because we should be disposed to doubt the observations of an individual engaged in experimental inquiries, but because the reasoning which he founds, or the principles which he deduces from important results, are occasionally far from being either legitimate or correct.

The experimentalist seldom commences his practical inquiries without having some preconceived view or principle to establish or refute, tending to direct these studies; and whatever be the nature of the conclusions, he is apt to seize and apply with avidity such only as are consonant with his own opinion. Although the reader is not liable to be blinded by the feelings and train of reflection which regulate the proceedings of the experimentalist, yet he is liable to be biassed by the plausible manner which generally accompanies the statement of simple facts, on account of the variety of views which are closely interwoven with them.

The experiments of Wilson PHilip, advanced to prove his peculiar opinions in regard to animal heat and digestion, are the best illustrations that can be brought forward to shew that experiments themselves, although correct, are occa- 
sionally more liable to mislead than even the deductions of theory, if they are regarded by a mind neglecting to generalize the facts presented, to note the relations between objects that are manifest, or to refer those phenomena, that have no obvious connexion to explain them, to the most probable or general law of the animal economy. This method of investigating the laws of the system has been entirely neglected by Wilson Philip, and to a certain extent by Brodie, and others engaged in similar physiological pursuits. The former gentleman divided the par vagum, and found, as a consequence of this operation, that the function of digestion was very much retarded or destroyed: from this effect he concluded, that digestion depends on nervous influence; but, what is somewhat extraordinary, while he observed the animal breathe with great difficulty-while he remarked its temperature to diminish 15 or 20 degrees,-nay, even after the death of the animal, when the lungs exhibited the colour and congested appearance of the liver-he never for a moment seemed to imagine that the proper performance of respiration, and the normal circulation of the blood, were con- 
ditions at all essential to the function of digestion. The same observations apply to the rest of his mutilations of the nervous system, in respect to animal heat and digestion. As the consideration of the former occupies the greater part of the following volume, I have attended eritically to the views of those who suppose the nervous system to be the efficient agent in maintaining the temperature of the body. I have not, on all occasions, performed experiments in order to prove the correctness of my own opinions; but have, when it was possible, taken the results of the experiments of others as data for my own reasoning. This plan is closely followed in several chapters, in which I attempt to shew the fallacy of principles advanced by Wilson Philip, Dr Edwards of Paris, John Hunter, and others.

I have not brought forward the varieties of opinion on every physiological subject treated in this work. My object has been to notice the views of those whose originality and importance merit repetition and consideration; nor have I been particularly desirous of developing minute details which naturally flow from general principles. 
Physiology, within a few years, has undergone material changes. There is scarcely a department of this science that has not been the subject of experiment or theory. The anatomy and physiology of the Nervous system have been particularly studied. Among the list of those who have distinguished themselves in this walk of science,' we cannot forego mentioning the names of Gall, Magendie, Legallois, Demoulins, Flourens, and Bell. Our own countryman may probably be considered as the most valuable contributor to our knowledge of the nervous system. His discovery of the difference between the nerves of motion and of sensibility belonging to the spinal cord and medulla oblongata has one property to recommend it, which places it pre-eminently above the contributions of his contemporaries: It has important relations to praclice. But although others may have been less fortunate in their scientific investigations, it is an incumbent duty to express admiration of the zeal and talent, with which they have devoted themselves to such pursuits; andit is equally a duty to acknowledge the benefits they have conferred, 
and the impulse they have given to experimental physiology.

It is not improbable that we shall continue to labour in the study of the nervous system long and patiently before we shall be capable of understanding satisfactorily its functions. Much has already been said and accomplished to dissipate the obscurities which hang over the phenomena it developes, but, at present, we possess only an outline of its important offices.

When physiologists knew less than they do at present, the production of animal heat was ascribed to chemical changes in the lungs, as it was observed that similar changes, occurring without the system, are accompanied by a disengagement of caloric; but since particular attention has been paid to the nervous system, others have advanced a variety of opinions and experiments, intended to prove that animal heat is a secretion, or that this system is intimately connected with its production. The former opinion has been promulgated and supported by Wilson PHILIP with ingenuity; and the latter, in our own country, has been fully developed by Brodie, with considerable diffidence and judgment. The 
mind, in taking a view of the different theories that have been advanced in order to account for the generation of animal heat, is continually confused by their variety and discrepancy.

It would be a waste of time to enumerate the various hypotheses that have been formed from the age of Hippocrates to our own. The first rational view was that stated by BLAck, in which he regards the respiratory function as producing changes upon the inspired air analogous to those attendant on combustion. This idea was eagerly adopted by LAvorsien, and was subsequently modified by him. The objection to the original view of BLACK is, that if the disengagement of caloric take place in the lungs, these would be consumed, or would become much warmer, from the nature of their office, than any other part of the body. This conse. quence has appeared so obvious, that no one, since the time of BLACK, has dared to support the same opinion. The experiments of $\mathrm{CRAW}_{\mathrm{R} \text { - }}$ FORD appeared to remove every difficulty which the subject presented. They seemed to prove that the capacity of arterial blood for caloric is superior to that of the venous. This circumstance 
would prevent the sensible heat of the blood in the lungs being greater than that of the rest of the system, as the quantity generated by the chemical changes is immediately lost in the greater capacity of the arterial fluid. A few years ago, Dr John Davy investigated the capacity of the arterial and venous blood, and his experiments are by no means favourable to the conclusions of Crawford. The difference of capacity between the arterial and venous fluids was observed to be so much less than what is stated by the latter gentleman, that the conservation of uniform temperature is scarcely to be explained by it. Indeed, the arterial blood is allowed by Menzies, Plenk, Davy, Magendie, Thenard, and many others, to be at least one degree warmer than the venous. If this be correct, it militates considerably against the doctrine of Crawford. Since the completion of this work, an idea has struck me, which appears sufficient, in conjunction with principles already stated, to explain satisfactorily the source of animal heat. It does not militate against the principles which have been developed in the present Experimental Inquiry. 
'Throughout this I have laboured to attain a knowledge of those conditions of the system which promote or retard the generation of animal heat, and these have little connexion with any particular theory. The present view may therefore be considered as an important addition to what has been already said on the cause of animal heat. The explanation I shall propose goes far to support the doctrine of BLACK, in which the increase of heat is attributed to chemical changes in the lungs. It is now, I believe, almost universally allowed, that the arterial is warmer than the venous blood, and it is more than probable that this result depends on chemical action. By taking into consideration, that a small quantity only of the air within the lungs is at any one moment deteriorated, and, still further, that the left ventricle contracts 70 or 80 times per minute, in order to propel the arterial blood which is transmitted by the lungs, we shall have reasons sufficiently ample to account for the possibility of these organs bearing such changes, and for the ease with which the system is supported in an equable temperature. If the body be supposed to possess 30 pounds of blood, and the heart to 
transmit at each contraction two ounces, and to contract 75 times per minute, we shall find that the whole mass of blood will pass through the lungs once every three minutes, or twenty times per hour. As it has been proved by direct experiment that the blood acquires at least one degree of heat in passing through the lungs, it necessarily follows, at this moderate calculation, that the system will receive 20 degrees of heat in an hour, or $\mathbf{2 4 0}$ degrees every twelve hours. If the respiration be accelerated, and the contractions of the heart be increased to 100 , the mass of the blood will circulate through the thoracic organs in one-fourth less time than is stated above, and consequently the temperature will be augmented one-fourth : the increase of one degree, instead of being repeated every three minutes, will be repeated every $2 \frac{1}{4}$ minutes.

According to the doctrine of Crawford, the evolution of heat is confined to the capillaries distributed throughout the body; but the present explanation of the manner in which the system acquires 20 degrees per hour, or 240 every twelve hours, is unfavourable to such an opinion, as it proves that the lungs transmit an 
immense quantity of sensible heat to the body,a quantity, it is highly probable, sufficient for every organic necessity.

From this view of the chemical changes in the lungs, it is apparent that the various internal parts of the system will possess, as nearly as possible, the same degree of animal heat. The blood which the left ventricle sends out at one contraction, is calculated to supply the deficiencies incurred by an equal portion which is returned to the right auricle; and as the whole circle of circulation is completed in two or three minutes, there can scarcely be a perceptible difference in the temperature of the different parts of the system. It is not my intention to give any opinion on certain minute points connected with the chemical theory. Concerning these, the most distinguished chemists are at variance; and, therefore, it would be presumption in me to offer even a conjecture on subjects to which I have not paid an exclusive attention. The present volume cannot be considered to contain a complete sketch of the Phenomena of Organic and Animal Life, employing this term agreeably to the division and reasoning of BICHAT, - but 
this incompleteness is to be attributed to the considerations which have induced the Author to postpone the publication of the second and last volume on the Phenomena of Organic and Animal Life.

I cannot conclude without making my warm acknowledgements to those Gentlemen who have assisted me in my Experimental Inquiries. I can but feebly express the zeal and ability with which Mr Hamilton, Surgeon, has on all occasions assisted me; nor must I forget to mention my obligations to A. T. Holroy D, Esq. Christ Coll. Cambridge, for the attention and talent which he devoted to some of the most important of these experiments. I am also materially indebted to George W. Chester, Esq. for many advantages which I have derived from his knowledge of minute anatomy, and of professional subjects. To Mr Kemp, Lecturer on Practical Chemistry, Edinburgh, and to Mr HARDX and Mr Murray, Medical Students, I have also to return my thanks for their kind assistance. 
ERRATA.

Page 30, line 11, for Foyer-read Floyer.

48, line 29 , for $100 \frac{3}{4}$-read $105 \frac{3}{4}$.

357 , line 21, for, and its derangement-read, and the derangement of the liver. 


\section{EXPERIMENTAL INQUIRY, \&C.}

\section{CHAP. I.}

Animal heat is not in the direct ratio of the quantity of oxygen inhaled, but in the inverse ratio of the quantity of blood exposed to this principle.

I. THE investigation of those laws that regulate the animal economy is at all times important, as a proper knowledge of these may enable us to support the healthy operations of Nature, or correct their occasional deviations. The subject of heat, from its extensive action and vital influence on animal and vegetative life, demands the closest attention. Many experiments have been performed to elucidate the difficulties which surround this department of science, and certainly much has been done towards removing them; but it is not presumption to assert that great obscurity still hangs over some of the most essential points.

II. It can scarcely be denied, that the functions of the lungs are instituted for the purpose 
of disengaging from the blood a portion of the excrementitious matter of the system, and also for the purpose of regulating the generation of animal heat. Although these principles are acknowledged to be correct in their general acceptation, it is still believed that animal heat does not bear an exact ratio to the number of respirations; and, moreover, by some it is advanced, that the nervous system is the source to which this must be either altogether or in part attributed. That animal heat is not proportionate to the number of respirations, is evident to every one that has paid any attention to the subject; but this consequence does not imply that it is not exclusively derived from the changes which the blood undergoes in the lungs, nor does it in the least favour the opinion that it is a secretion,* or that it is to be ascribed to the nervous system, agreeably to the opinion of Mr Brodie.

III. Respiration is composed of two acts, inspiration and expiration : the former is accompanied by an enlargement of the chest and renewal of atmospheric air; the latter, by the approach of

* "The experiments related in the last chapter, compared with those on secreting surfaces, seem to me to prove, that the caloric, which supports animal temperature, is evolved by the same means, namely, the action of the nervous influence on the blood, by which the formation of the secreted fluids is effected, and, consequently, that it is to be regarded as a secretion." - An Experimental Inquiry into the Laws of the Vital Functions, by A, P. Wilson Philip, M. D. F. R. S. E. 
the parietes of the thorax, and the expulsion of the deteriorated air.

IV. The object I have in view in the present chapter, is to shew, that these two acts of respiration have each an independent function, which is continually regulating the distribution of the blood throughout the system, and consequently producing considerable modifications in its circulation in the lungs. The principles, if found satisfactory, will explain, in a beautiful and consistent manner, a variety of phenomena intimately related to organic and animal life.

V. Physiologists have patiently investigated the changes which the air experiences during respiration, the conditions of arterial and venous blood, and the influence of internal and external means in diminishing or increasing the generation of animal heat, but have neglected to ascertain in what way this principle is modified by the different proportions of blood existing at different times roithin the chest.

VI. This omission is somewhat extraordinary, but it is principally to be referred to two causes; first, the belief that the circulation of the sanguineous fluid is continuous and equable; and, in the second place, to the nature or direction of the experiments, the greater part of which was confined to the lower animals. That the blood is at all times in motion in every part of the body, cannot be called in question; but it is far 
from being regular either in velocity or quantity in any given point, even for a series of hours, Many experiments were made on the lower animals to elucidate a few of the above observations, but, by attending to these, it was almost impossible to appreciate the precise influence of inspiration and expiration upon the system. Except by direct experiment this could be ascertained only by repeated observations on man himself, when excited or depressed by the emotions of the mind, as it is then that these powerful states exhibit each of these acts in its extreme developement and its subsequent results.

VII. The experiments of Dr BARRY* fully prove that the blood during inspiration circulates with freedom through the lungs, but that during expiration the contrary is the case.

Dr Bostock, in alluding to these experiments, observes, "The obvious objection to such experiments in the abstract is, that they apply to what occurs in extraordinary states of the respiration rather than to its ordinary action, and that, in the healthy condition of the system, the different states of the thorax cannot be perceived to affect the pulse." With these remarks of this distinguished physiologist I cannot agree.

VIII. In the first place, if, in a violent inspira-

* Vide Experimental Researches on the Influence of Atmospheric Pressure upon the Circulation and Absorption; 8vo, London, 1826. 
tion, the blood ascends in a tube attached to the jugular vein of an animal, it proves, even when this act is forced, that this is its natural tendency, or otherwise it would not occur; and if we conceive the inspiration of a milder character, the fluid will rise less, but it will not fail to shew its ordinary disposition. He also observes, that " the different states of the thorax cannot be perceived to affect the pulse." This is very true; but it is no objection. The alternate motions of the thorax are obvious, and the pulse is found regular when those are in their natural condition, but whenever these are in any way disturbed the former is immediately deranged, shewing that the regularity of the pulse depends on the regularity of the alternate motions of the chest, and not that it is uninfluenced by them. By taking a few deep inspirations for a minute, I can raise the pulse ten beats in that period, diminishing proportionably its strength; but as soon as the two acts of respiration are restored, the pulse returns to its usual condition.

IX. The early Physiologists endeavoured to demonstrate the opinion which Dr BarRy supports; and this is the view that Dr CArson has given in his work on the Motion of the Blood, published in 1815. "The motion of the blood, while it flows in the veins, is produced by the force of the heart and arteries urging it behind; by the $a b$. straction of a share of the atmospheric pressure from 
it in front, in consequence of the resiliency of the lungs interposing its influence in the intervals between the contractions of the heart; and by gravity, which is rendered available in this case by the projection of the arteries and the diastole of the auricles." The reader will do well to peruse with great care this ingenious work: its merits have never been sufficiently estimated.

Mr Charles Bell* endeavours to shew that Nature has been particularly solicitous topreserve the act of inspiration, as this is "secured by a double provision of nerves, viz. those which come out from the sides of the spine, and those which descend from the neck, when the act of expiration is provided for solely through the former." He still farther says, "These nerves, which govern the muscles of inspiration, are linked more intimately by sympathy with the state of circulation and respiration; for we see in disease, as in experiments on animals, that, when the powers of life have run low, the sympathy is still exerted with such sudden catchings of the muscles of inspiration, and with an effort so powerful and unexpected, as to startle, while the expiration is soft and without effort," sc. + "These considerations exhibit the importance of the act of inspiration over that of ex-

* An Exposition of the Natural System of the Nerves of the Human Body, p. 239.

+ Ibid.p. 241. 
piration, and prove the necessity for those exterior nerves of respiration."*

$\mathrm{X}$. I have not brought forward the above extracts as additional authority to strengthen my opinion, but for the purpose of shewing that his ideas in regard to the phenomena of inspiration and expiration are not consistent with more enlarged views on this subject. The sympathy of which he speaks, existing between the nerves which govern the muscles of inspiration and the state of circulation and respiration, arises from the painful anxiety occasionally felt from accumulation of blood in the lungs, whether from mental emotions, fatigue, or disease : and as this state of the chest is accompanied by the sensation of dissolution, the individual makes repeat. ed involuntary inspirations, and these inspirations are proportionate in degree to the severity of the cause and the strength of the constitution. The sudden catches are referrible to acute and instantaneous sensations, produced by conditions in the mode of circulation which are incompatible with the continuance or well-being of life. The nerves appear more intimately linked by sympathy to the state of the circulation and respiration, because, in those extreme cases of inspiration to which he alludes, the action of the system is so oppressed that the means which Nature employs

* Ibid.p. 242. 
to overcome it, or which are occasioned by it, must necessarily disturb " the state of circulation and respiration" to a more obvious extent than expiration. The latter act is "soft and without effort," not because it is deficient in certain nervous fasciculi, but because it is not excited by those momentous and painful occasions which characterise the former. The act of coughing, and the violent state of respiration after running, shew, whenever there is the necessity for expiratory motions, that these are performed in a manner as manifest as the inspiratory, which proves that their character is not "soft and without effort," from the cause assigned by Mr BELL.

XI. Inspiration having the tendency to increase the quantity of blood brought to the lungs, it follows, that if these preponderate, either in number or perfection, over the expirations, a greater proportion than usual of this fluid will be circulating in the pulmonary tissue. The truth of this is apparent in all instances in which these acts exert a superior and almost exclusive energy. In the depressing passions of the mind, particularly in fear, and in certain diseases, as spasmodic asthma, a state of congestion, or unusual determination of blood to the lungs, is present. This is indicated by the very quick and almost imperceptible pulse, palpitation of the heart, and diminution of temperature. 
XII. The chief phenomena of fear, and of every state of mind approaching to it, are composed of deep inspirations; and these being more numerous or complete than the expirations, the blood gradually ceases to flow to the surface of the body and the extremities, making the former pale and the latter cold ; and this fluid, of necessity, is retained in the abdominal and thoracic viscera; these, containing more than is proper for the operation of their function, the pulse is observed to loose its fulness and strength, and to become weak and frequent. This change is in accordance with the condition of thelungs, which, having more more blood of a less stimulating quality than what is natural, must make a greater demand upon the action of the heart, and, when this is so great as almost to overwhelm it, palpitation is the consequence; but when the demand is less, the heart is enabled to transmit the blood it receives, which, however, as will be hereafter explained, is deficient in its ordinary stimulating qualities, and therefore does not excite the strong and full contractions of this organ; but the necessity of continuing its action being imperative, the heart performs, in frequency and feebleness, what is previously accomplished in slowness and energy.*

XIII. This peculiarity of the pulse, agreeably to certain alterations in the functions of

* Vide Appendix for a full explanation of this and the preceding section. 
the lungs and heart, is not confined to depressing emotions of the mind, or to any single disease, but will occur whenever the circulation of the sanguineous fluid becomes becomes rather internal than external, ${ }^{*}$ whether this be occasioned by exposure to cold or by affections of an asthenic description.

XIV. An expiration is an act by which deteriorated air is expelled : but this is only one part of its important function. It is the effect of inspiration to facilitate the motion of blood through the lungs; but it is that of expiration to retard it. If, then, we have a preponderance of expiratory actions, we diminish the quantity of blood generally circulating through the respiratory organs; and this being the case, we shall observe the heart beating with unusual vigour, at times amounting to palpitation, the pulse quick and strong, the countenance flushed, the whole surface of the body exhibiting improved circulation, and increase of temperature.

XV. I cannot agree with Mr Charles Bel.L in thinking that Nature has been extremely solicitous to secure the act of inspiration, and that expiration is of little importance comparatively. He draws his conclusions from observing the distribution of nerves ; and mine also are the result of observation, but not directed by any desire to appreciate the value of nervous fasciculi,

* These terms are explained in Chap. IV. 
but rather to note the multifarious changes belonging to each act, and, from the results which they present, to estimate their respective importance, and the intentions of Nature. From pursuing this mode of investigation, it is not difficult to prove, that, whenever inspirations exceed, either in number or completeness, the contrary states, whether in disease or mental trouble, the powers of life are invariably depressed, and occasionally endangered; but whenever expirations possess a similar superiority, with few exceptions, the system receives an additional stimulus, the assimilating functions are invigorated, the mental faculties are excited, and, in fine, the powers of life are augmented. If this statement be correct, we are necessitated to acknowledge, that the solicitude of Nature, according to the opinion of Mr Charles Bell, has been rather to the injury than the benefit of the constitution.

XVI. The lungs, from their beautiful structure, are every way adapted to admit of great and minute distribution of blood, and also for the reception of a considerable quantity of air.

The following description of the lungs, by Monro, gives an accurate idea of the provision which is here made for exposing a large surface of blood to the action of the air. "As our lungs in a full inspiration contain about 220 
cubic inches of air, the whole internal surfaces of the lungs will be nearly equal to 440 square feet, or nearly thirty times greater than that of the whole external surface of the body."*

From the little already stated concerning the action of inspiration and expiration, it is manifest that the proportion of blood and air is subject to great variations; and it is my intention to explain more particularly the nature and origin of these.

XVII. It is not easy to ascertain the quantity of air which is evolved at each ordinary expiration; this is so materially modified by the state of the mind, system, and capacity of the chest, that great discrepancies unavoidably exist in the results of the most distinguished chemists. But the data which I shall presume to establish, and the reasoning which will naturally flow from them, will be little affected by the varieties of opinion.

Dr Bostock has paid considerable attention to the subject of respiration, and his contributions are neither few nor unimportant; and, on this account, I shall draw my deductions from the experiments of this talented individual.

XVIII. By an ordinary expiration, $40 \mathrm{cu}-$ bic inches of air are emitted; but after this we are enabled to expel a considerable portion, and this quantity is estimated at 160 or 170 cubic

* Monro’s Elements of Anatomy, Vol. ii. p. 95. 
inches, "so as to give 200 or 210 cubic inches as the difference between the states of ordinary inspiration and of forced or extraordinary expiration." As it is impossible to empty the lungs completely, many calculations have been made concerning the quantity of the residual air which must necessarily remain after violent or long continued expirations ; and this most probably amount to 120 cubic inches.t From the above statement, it is clear that, after an ordinary expiration, 280 or 290 cubic inches remain in the lungs, in other words, 7 or $7 \frac{1}{4}$ of ordinary inspirations. What is the intention of Nature in giving to the lungs a capacity sufficient to contain almost 300 cubic inches of air after the evolution of that portion which is no longer fit for the animal economy? The explanation of this intention will throw new light on many mysterious changes of the system.

XIX. The air which is received by any single inspiration, does not immediately act upon the blood; its office is to supply the deficiency occasioned by the previous expiration; and after several series of such expirations it is then brought into intimate contact with this fluid, and tends to continue the successive alterations which it undergoes.

XX. The organization of the lungs allows a

* Bostock's Elementary System of Physiology, Vol. ii.p. 25. + .Ibid. p 29, 
vast quantity of blood to circulate at the same moment in them; and the same adaptation favours, in like manner, the more general diffusion of the air; so that, in the usual operation of the pulmonic functions we may conclude that about 300 cubic inches of air are acting simultaneously upon this fluid; and, still further, that the expiration of 40 cubic inches is the whole of that portion which is rendered unfit for the purposes of life by the action of the blood. It is impossible to suppose, that air is deteriorated immediately on its being inspired; if this were allowed, the whole quantity which belongs to the lungs would be unfit for its vital function, as this must be contained in the air-cells and subject to the influence of the capillary vessels, and therefore placed under circumstances that would deprive it of its properties.

$\mathrm{XXI}$. This contrivance is a wise provision of Nature, as it enables an individual to speak, laugh, run, in fine, to accommodate himself to any kind of exercise where expiratory actions are required, without immediately affecting the office of the lungs, which would necessarily be implicated if the inspired air were instantaneously appropriated to those changes which are indispensable for the continuance of life.

The short breathing which an individual exhibits after violent exercise, as after running, is 
not occasioned by any condition of the heart or circulation, as is generally supposed, but arises from the diminution of the ordinary and stationary quantity of air belonging to the chest, and it ceases as soon as the repeated inspirations have re-established the order of nature.

XXII. I now propose to show in what way " animal heat is not in the direct ratio to the quantity of oxygen inhaled, but in the inverse ratio to the quantity of blood exposed to this principle."

As the blood is not immediately acted upon by the fresh air which is received, it follows that the alterations are to be referred to that already existing in the pulmonary cells; and, moreover, it is natural to suppose, if we diminish the quantity of blood, that the chemical changes will be more complete, because the air will have a less portion to oxygenate.

In the words of Haller, "the effects of expiration are, a compressure of the blood-vessels in the lungs, a reduction of the bronchia into more acute angles, a pressure of the reticular small vessels by the weight and contact of the adjacent larger vessels, and an expulsion of the corrupted blood from the lungs, by which means part of the blood hesitating in the capillary arteries is urged forward through the veins to the left side of the heart, while, at the same time, that part of the blood is resisted which flows in by the artery from the right ventricle. Expira- 
tion, therefore, will stop the easy passage of the blood through the lungs."*

XXIII. The above description is sufficiently correct and explicit to shew, that the circulation of the blood is impeded in expiration; and, therefore, if that which is returned by the veins is partly obstructed in its passage, the obvious consequence is, that the quantity in the lungs will be diminishing more rapidly than the quantity of the venous blood necessary to maintain the ordinary balance between the two systems can be supplied. If we suppose that the lessened capacity of the chest simply retards the flow of blood from the veins, without in the least considering that this diminution facilitates arterial circulation, the lungs will contain less blood than is found to exist in the well-poised states of respiration. The lungs, as stated in XVIII., always contain a considerable quantity of air; and as this is that portion which immediately acts upon the blood, if we diminish this blood by a series of expiratory actions, it is philosophical to suppose that it will be more perfectly oxygenated, because a smaller quantity is submitted to the influence of that important agent.

XXIV. I have observed, in speaking of the quantity of air contained within the chest, that expiration can lessen this only to a determinate

* Halleg's First Lines of Physiology, Vol. I. Sect. cclxxir. 
extent. The same remarks will also apply to the blood circulating in the lungs. If we consider the favourable disposition of this organ for the reception of the vital fluid, we shall be persuaded that the portion which is transmitted by the right ventricle is not that portion which is immediately expelled by the left: several times this quantity must be exposed to the action of the air: and it is probable that this has its degrees of oxygenation, according to the rank which it occupies in the series of changes or pulmonic capillary circulation. This view explains why the causes, which prevent the free return of blood to the lungs, do not deprive the heart, in the same ratio, of that which flows along the arteries. If the heart depended on this delicate adjustment or harmonious action, its function would be continually liable to derangement, not only on extraordinary occasions, as at present, but almost on every trifling occurrence affecting the system.

XXV. The blood possessing more stimulating qualities when expiration preponderates, the heart participates in this improvement; its contractions are more vigorous, from its cavities being excited ; and, therefore, when the acts of expiration have become more moderate, or when their exclusive character has subsided, the arterial system retains, for some time, a superior energy of function, indicated by fulness or 
strength of pulse, forcible contraction of the heart, more equable distribution of blood, and an augmentation of temperature.

XXVI. The heart having its action increased by the improved qualities of the blood, communicates this condition to every part of its system; the capillary vessels and the veins almost instantaneously participate in it ; and if muscular exertion be conjoined with this, the return of the blood is as much facilitated by these means, as its transmission is by the heart. The extremities and the surface of the body receiving more of this fluid, as is evident from sensation and external appearances, the abdominal and thoracic viscera must possess less, and therefore the quantity of blood circulating in the lungs is not so great as in the sluggish state of the body, consequently it will be better oxygenated. The explanation of these phenomena enables us to account for others that have hitherto been considered the most mysterious of the system, such as the presence or absence of the buffy or inflammatory coat in diseases, and in moderate or excessive exercise. If a local disease becomes highly painful, or irritates the neighbouring parts, the circulation is disturbed, and the heart and lungs are quickly implicated in the disorder, and they soon establish, throughout the system, the various symptoms of fever, in intensity or 
kind proportionate to the cause and the state of constitution. In the congestive or real typhoid fever, the inflammatory coat is seldom present; nor should we expect this, if we consider the collapsed or cadaverous condition of the body in this disease: the organic and animal functions are universally deranged,the liver and the spleen, for example, are engorged; - vomiting, headache, delirium, the small, frequent, and weak pulse, the diminution of temperature, the great coldness and almost insensibility of the extremities, and, lastly, the petechiae, maculae, or vibices, express more eloquently than words can describe, the impoverished properties of the blood, and the cause to which these changes must be attributed, viz. the congested state of the lungs and internal organs, on which account the blood is imperfectly oxygenated, because the inspired air has to act on a quantity much greater than circulates in the pulmonary cells, during inflammatory affections, moderate exercise, or health.

XXVII. As I purpose, in a short time, to publish a work on Fever and Inflammation, I shall not at present pursue farther an investigation into the nature and origin of the above phenomena. From the view which is developed, it is clear that the state of the blood, in disease, may mislead very much in the treatment, unless we be acquainted with those causes on which its 
character depends - which knowledge, I regret to say, has not hitherto been possessed by the medical practitioner; the approaches which he has made have been made by means of empiricism, which occasionally teaches what is right, but too often misguides by its fallacies.

XXVIII. Having explained, and, I hope, established these principles, we proceed now to illustrate them more fully, by a variety of other applications. Since the lungs are subject to great variations in the quantity of blood which they receive, and since these variations influence the generation of animal heat, we may state the following laws as universal:

1. That, in every case, in which inspirations preponderate, either in number or completeness, the pulse will be small, weak, and for the most part frequent : the surface and extremities of the body pale or collapsed, and the temperature of the system less than in the regular action of the two states of respiration.

2. In every instance in which expirations exceed inspirations, either in number or completeness, the pulse will be strong and frequent, the surface and extremities of the body florid and full, and the temperature of the system higher than natural.

3. The influence of cold upon the surface of the body will diminish the generation of animal heat, according to the degree of congestion produced in the internal viscera.

4. The influence of heat applied to the same parts, will increase the temperature of the body, according to the 
relief which it gives to the internal organs, by diffusing more generally and equably the circulation of blood.

5. The buffy or inflammatory coat of blood, in diseases and exercise, will be proportionate cateris parib:is to the quantity and force of blood circulating through the lungs, as its changes bear a ratio to those conditions.

6. The absence, or partial existence of this symptom, in typhoid or nervous affections, will be regulated by the quantity and force of blood circulating in the lungs ; but these states will be most obvious when the quantity is the greatest, and the force the least.

\%. Every exertion characterised by muscular and expiratory action will increase the temperature of the body, and tend to equalise the circulation.

8. Every exertion characterised by muscular and inspira. tory action will diminish the temperature of the body, and tend to destroy the regular distribution of the blood.

9. The effects of respiration, on the animal economy, can be estimated only by ascertaining the character, frequency, and force of the acts of which it is composed.

XXIX. Legallois found, in his ingenious experiments on rabbits, that, when they were tied upon their back, or in any way constrained, the temperature of the animal was diminished. He afterwards endeavoured to discover whether a smaller portion of oxygen was consumed during this state, to account for the diminution. Experiments instituted for this purpose require 
great care and exactness on the part of the operator, to enable him to draw legitimate conclusions; indeed, from their delicate nature, they are liable to several sources of error. The re. sults of his experiments are not uniform, but the greater number seem to shew that less oxygen is consumed when the animal is constrained. "It was found, however, that, in certain cases, the cooling was more rapid than ordinary, even when more oxygen is consumed, owing (as the author conjectures,) to the struggles which are made carrying off a portion of the heat."*

It is possible, when an animal is placed in these circumstances, that less oxygen may disappear, but this is not the cause of the phenomena; these will be explained by referring them to a more general and important principle, viz. the character of the respiration and the consequent condition of the circulation. When an animal is put upon its back, the palpitation of the heart is perceived, and the state of the breathing is extremely hurried, from mental influence. The troubled action of the heart shews it is oppressed by the quantity of blood which is determined to the lungs, occasioned by the deep inspiratory acts of respiration; and these possessing more than they are accustomed to present to the agency of the air, it therefore follows that the p. 273 . 
animal temperature is affected, not from the want of oxygen, agreeably to his opinion, but from the inefficiency of this to act on an augmented quantity of blood. The truth of this view is proved, according to my reasoning, in the circumstances connected with the latter part of the above sentence which I have quoted. When the animal made struggles, LEGA LLoIs observed that the quantity of oxygen consumed was greater than usual at the time that the temperature was falling rapidly, and he conjectures that a portion of the heat was carried off by these efforts. If LEGaLLors had reflected. on this subject, he would have perceived the evident fallacy of this explanation. In every case of muscular exertion, accompanied by an increased consumption of oxygen, heat is generated; and although this may be carried off more quickly, and to a greater extent than when the body is at rest, still these conditions are not sufficient to counterbalance the quantity which is generated.

XXX. From the nature of the greater part of the experiments I have performed on animal heat, I have had every opportunity of verifying the preceding remarks, and I have always found the character of the respiration and the action of the heart as stated in XXIX. Among the num. ber of rabbits which $I$ kept in the rooms appro. priated to experiments, there was one which had 
always been extremely timid : it was white and full grown. From exhibiting great trepidation on even a distant approach, I proposed to select this for the purpose of ascertaining the influence of the mind on the production of heat. I made an attempt to catch it, but was unsuccessful, as it almost immediately escaped into a situation inconvenient to enter ; but, while here, I observed well the great agitation of the body, such as I had not seen before, nor have I since. This animal, almost directly, lost its fine healthy appearance and appetite; it partook of nothing after this accident, and died in two days. On dissection, the lungs were found to exhibit well marked symptoms of inflammation, the liver was much enlarged and congested, and the intestines were, in certain parts, highly vascular. The stomach was full of meat, which it had taken before this occurrence, but which was scarcely, if at all, acted upon by the gastric juice. From the singularity of this case, I presented it to the Edinburgh Hunterian Medical Society. Among the crowded attendance of the evening was $\mathrm{Mr}$ Lrzars, the distinguished anatomistand surgeon. $\mathrm{He}$ and the other members examined the diseased parts with care, and agreed with the above observations. This case, although an extreme one, illustrates in a very striking manner the opinion I have endeavoured to support regard. ing the changes in the distribution of the blood, 
from struggles or other causes, attended by inspiratory actions.

XXXI. The opinion is also much strengthened by the experiments and observations of $\mathrm{Dr}$ Currie. The first individual which he subjected to cold immersion was Richard Edwards, whose temperature was very soon and considerably diminished. "To my surprise," he says, "although the attendants were rubbing him dry with towels, during this time the mercury fell rapidly. He was put into a warm bed, and his heat, when examined under the tongue, was $87^{\circ}$, at the axilla $89^{\circ}$. Frictions were used, and brandy mixed with water administered; but I found on this, as on all future occasions, the best mode of counteracting the cold was to apply a bladder with hot water to the pit of the stomach, a fact which I think important: This being done, his shiverings, which before were severe, soon ceased, and he became more comfortable.*"

In another experiment on one Richard Sutton, Dr Currie remarks : "In the last experiment, when the heat sunk rapidly, Sutton said that he felt a coldness and faintness at his stomach, which he had not perceived before, and when I felt the motion of his heart it was feeble and languid. In some future trials of the effects

* Medical Reports on the Effects of Water, cold and warm, as a Remedy in Fever and Febrile Diseases.-Appen dix, p. 26. 
of immersion in fresh water, the same coldness at the stomach preceded a rapid fall of the mercury; and these facts, together with the effects I found from applying a considerable heat to this part when the body was chilled with cold, convince me that there is some peculiar connection of the stomach, or of the diaphragm, or both, with the process of animal heat."*

This peculiar connection, to which Dr Currie alludes, has nothing whatever to do with the direct generation of animal heat. The state of the heart and pulse, and the relief which the individual experiences on the application of warmth to the pit of the stomach, show that the heart and the lungs are oppressed by the congestion of blood, and that the stomach is also in the same state. From the situation of the lungs and heart, it is impossible to apply any means so as immediately to influence their functions; but, in regard to the stomach, liver, and spleen, the contrary is the case ; and as these are engorged in the same manner as the thoracic viscera, the direct application of heat produces instantaneous effects in them, and the improvement is transmitted to the others less favourably circumstanced. The nature of this improvement is accelerated circulation; the blood is evacuated from the parts to which the bladder is placed, as is always the case

* Ibid. page 35.-Appendix. 
in congestion when the capillary and larger vessels have their action increased, whether by heat, galvanism, or stimulants ; and these parts being, in some measure, disburdened of their own individual load, are prepared to take off a portion from the lungs and heart; or the excited action of their vessels is communicated to those organs; and from this cause, or rather from the combination of the two, the respiratory function speedily regains its ordinary degree of activity, and re-establishes throughout the system the effects of its invigorated energy.*

XXXII. It is imagined by some authors of distinction, that an explanation founded on physical principles is insufficient to account for the phenomena: they are disposed to attribute the beneficial consequences to nervous influence or connection; but as they have not yet favoured the world with reasons, beyond mere surmises or hypotheses, we must adhere to that explanation which is strengthened by so many obvious effects in close relation to the cause assigned, that it is almost impossible to observe one without becoming acquainted with the other.

* Dr Kelxie, some years ago, proposed the attempt to stop the cold stage of intermittent fever, by applying the tourniquet to the inferior extremities, and it has certainly been successful.-The ancients also employed ligatures for the same object, and in a similar manner. The former was the suggestion of physiological principles, the latter of empiricism. 
XXXIII. Dr Edwards, in his valuable work, "De l'Influence des Agens Physiques sur la Vie," brings forward many instances to shew, that animal heat is not evolved in the direct or any proportionate ratio to the number of respirations. In speaking of birds, in which he made a variety of experiments, he observes, "Quand ils sont trés-près de l'âge où ils peuvent soutenir leur température à l'air, voici ce que j'ai observé chez plusieurs individus dont j'ai donné les observations detaillées dans les tables; un d'eux avait $40^{\circ}$ et $90^{\circ}$ inspirations par minute. Retiré du nid, et exposé à l'air de l'appartement, qui etait à $18^{\circ}$, il perdit dans un quart d'heure 3 cependant sa respiration c'etait accélérée. Il arriva à 120 inspirations qui se soutinrent pendant vingt-minutes. Il s'etait alors réchauffé d'un demi-degré; quelque temps après il se refroidit de nouveau; mais sa respiration, qui etait devenue un peu moins frequente, acquit de l'ampleur; sa chaleur se retablit de la même quantité, et persista long-temps à ce terme." *

In attempting to explain these variations, he does not seem sensible of the change which the blood has undergone in its distribution affecting the generation of animal heat. He regards the augmented respiration as favourable to the increase of this principle, as "multipliant le

* Dr Edwards, p. 510. 
contact de l'air avec les organes les plus propres à ressentir son influence vivifiante."*

XXXIV. It is evident, that the increased respiration is not an effort of nature, as generally supposed, to counteract the injurious agency of the cold, but arises from the accumulation of blood in the lungs and other internal organs, which occasions a difficulty of breathing, giving it a laborious and inspiratory character; and therefore the tendency of this action is contrary to the one taught by himself and others.

In the subsequent chapters I shall develope more fully these views, and shall also point out several fallacies in the general principles of this able physiologist,-fallacies which do not in the least impugn the correctness of his experiments, but expose the inaccuracy of those premises whence he has drawn his most important conclusions.

XXXV. I have given several instances in which increase of temperature is invariably the result of muscular and expiratory action, but have not yet adduced one in which there is a diminution from muscular and inspiratory action. The best example of the latter is asthma. The individual who is subject to this spasmodic affection seeks, as soon as the symptoms approach, a free current of air, from the painful sensations of dissolution which accompany this disease.

Ibid. p. 300. 
When the fit is very severe, the exertions of the patient to inspire are extremely urgent; the chest and shoulders are frequently and violently elevated, exhibiting, in an obvious manner, deep and almost continued inspiration. The blue lips, pallid countenance, collapsed features, small, quick, or almost imperceptible pulse, and great diminution of temperature, are of themselves characters sufficient to corroborate the justness of the principles propounded.

Sir JoHn Foyer says, "The scapular and lumbar muscles which serve for strong inspiration join all their force, and strain themselves to lift up the breast and shoulders for enlarging the cavity of the chest, that the lungs may have a place sufficient for their expansion, and the air be more plentifully inspired."

Dr Bree, who was subject to this species of asthma, and who has also written an excellent work on this disease, observes, that the animal temperature is materially affected by it. $\mathrm{He}$ has sometimes found it as low as $82^{\circ}$ during the paroxysm.

XXXVI. If the frequency of respiration in all cases produced animal heat, should we not expect that the function, when accelerated to its greatest extent, would evolve a proportionate quantity of this principle? But in this example we perceive that a quantity of air, much greater 
than what is natural, is brought into contact with the blood in the lungs, and yet the temperature is less than in any other disease or state of the system.

XXXVII. The intentions of Nature are not at all times easy to divine. In the rude researches of science, the means by which she acts, and the object she wishes to attain, are seldom correctly recognized; but whenever our knowledge respecting the instruments she employs becomes accurate, it is easy to appreciate the applications she has in view. How wisely, then, is it ordained, that, in the earliest years of existence, the life of the infant, from the variety of its wants, the delicacy or sensibility of its constitution, and from the many affections and changes to which that age is subject, may be truly said to be composed of cries, tears, and restlessness! To ease the child, and secure our own peace and comfort, our efforts are directed to appease these disagreeable complaints ; but it is fortunate that our interference is not at all times successful. At the time when these symptoms are present the future man may be considered still in embryo, and the developement of this being is regulated by the activity and extent of organic functions, which are adapted to unfold and maintain the various powers of theindividual. It is therefore instituted that these functions shall be more than usually vigorous, when present and future necessities 
demand attention. The frequent cries of the infant are made at the expence of expiratory actions, and the continuance of these renders the blood stimulating, the circulation more equable and quick than at any other period; and these principles being established, every organ of the system soon participates in the primary improvement, and the reciprocal influence is generally diffused and felt throughout the system.

XXXVIII. In infants whose constitutions are strong, we sometimes observe the facility with which they are nursed; and these being well formed, and of sound health, do not require the operation of extraordinary means : but those in an opposite state, from their great necessities, are continually excited by causes calculated to lessen or remove the sources of irritation. It is no objection to this view to advance, that many diseases are to be traced to these actions which I regard as salutary. The heart is sometimes subject to aneurism, from having been too much excited, either by external or internal causes, by means of which too much blood, or blood of too highly oxygenated properties, is determincd to this organ ; but these consequences are no objections to its utility.

XXXIX. If the nature of this chapter would allow me to pursue the train of the present argument through the variety of its relations, it would not be difficult to point out or explain 
many other efforts of Nature, efforts which are treated as diseases, because they disturb the ease and diminish the enjoyment of life. Few things are more disagreeable than a cough; but I am persuaded, from the character of its action, that it is rather a blessing than a curse in many chronic affections, except when violent; and many acute states of the system are carried off by this timely agent, particularly those which precede inflammation, or which succeed the severity of its symptoms, when the capillary vessels have lost their usual tone and energy of circulation. The precise mode of its influence will be better understood when we come to the subject of the action of emetics. In concluding, I may remark, that it is probable that more die for want of a cough than of a cough : the idea is strange, but it is a legitimate conclusion from facts or premises that have been carefully considered. 


\section{CHAP. II.}

\section{The Cause of Animal Heat.}

XL. IN entering upon the investigation of the present subject, I am not insensible to the many difficulties by which it is surrounded. I have not only to contend against the natural intricacy or delicacy of the pursuit, but have to oppose my humble opinion against others, which have acquired authority and reverence from the talents and respectability of those, who have trod in the same path; but, as I am stimulated only by the desire of arriving at truth, opinions, and not individuals, are to be considered.

The following points are those to which I intend to pay particular attention, and whose correctness I shall attempt to prove :

1. Animal heat is proportionate to the capacity and activity of the lungs, not understanding by activity the number of respirations, but the number and character of its compounded acts, inspiration and expiration.

2. This principle is proportionate to the chemical changes in the lungs, and the perfection of all other functions is also in the direct ratio to these.

3. The nervous system has no influence whatever upon the generation of animal heat, except in diminishing or retarding those chemical changes on which it depends, by destroying the natural proportions of blood submitted to the action of the air. 
4. The results of artificial inflation cannot be considered as making even a distant approach towards those chemical changes which occur during the natural state of the system.

5. The operation of poisons on the nervous system, accompanied by a diminution of temperature, is no proof that this principle is derived from the action or influence of this system, except in so far as its derangement disorders the harmony of chemical laws operating in the lungs.

6. The consumption of different quantities of oxygen, or the evolution of different quantities of carbonic acid, cannot, as is the received opinion, be regarded as a criterion of the temperature of an animal, because " animal heat is not in the direct ratio to the quantity of oxygen inhaled, but in the inverse ratio to the quantity of blood exposed to this principle."

XLI. In support of the first proposition, it is obvious that, in every kind of muscular exertion, attended by expiratory actions, the temperature of the body is augmented; and I have shown, in the preceding chapter, that this arises from the quantity of blood in the lungs being less than in the unexcited state of the system, not only at the commencement of the invigorated action, but throughout the continuance of its energy.

XLII. If we diminish the respiratory action, we lessen the generation of animal heat.

XLIII. If the circulation of blood in the lungs and internal organs be accelerated by c 2 
warmth applied to the surface of the body, or by the application of friction, heat is generated in increased proportion, because the lungs, by these means, are indirectly relieved of a portion of blood, either natural or foreign to their normal function.

XLIV. If the circulation of blood in the lungs be impeded by an increased quantity being determined to them, as by the depressing passions of the mind, by the paroxysm of spasmodic asthma, nausea, cold, and soporific medicines, the evolution of heat is proportionately lessened.

XLV. In the different inflammations and fevers of the system, the blood, with few exceptions, has the buffy coat proportionate to the degree of disturbance in the respiration and circulation. It is found, from experience, that inflammation of the thoracic viscera exhibits this appearance in a more marked manner than the same occurring in the abdomen. This is to be attributed to the greater disorder of the respiratory functions; but these are sometimes as much deranged by acute diseases of the latter cavity as by their own affections; and when this is the case, the appearance of the blood is of the same character.

XLVI. The buffy coat is seldom or never present without having been preceded or accompanied by an augmentation of animal temperature; in fine, its extent is generally observed to bear a ratio to the increased temperature, and 
this as invariably is proportionate to an excited state of the respiration and circulation.

XLVII. In the various diseases of the thorax and abdomen, characterised by congestion or sluggishness of action, we have an opposite train of symptoms : the blood is not of a highly florid aspect, nor does it exhibit the buffy coat; the surface of the body is cold, collapsed, pale, or discoloured, and every internal function is affected by a diminution of its regular action, and at this time the animal temperature is much less than natural ; proving, as evidently as the relation between cause and effect can possibly do, that the lungs are the organs which influence the properties of the blood and the evolution of animal heat.

XLVIII. If further proofs were required, we might allude to the temperature of different animals, which is high or low according to the amplitude of the respiratory functions : but this is a subject so often discussed, and so well esta. blished, that it requires little attention here.

XLIX. The nervous system has no influence whatever upon the generation of animal heat, except in diminishing or retarding those chemical changes on which it depends, by destroying the natural proportions of blood submitted to the action of the air.

The experiments and views of $\mathrm{Mr}$ Brodie are those generally alluded to, to prove that animal temperature is rather the result of nervous influence than of chemical changes. The re- 
spectability and talents of this gentleman attach consequence to his physiological investigations; but his contributions connected with the present subject are far from being satisfactory. He has the credit of having presented a number of facts, new, interesting, and important to the profession; but, in the endeavour to explain these, he has unfortunately overlooked obvious causes, and has attempted to ascend to others of a mysterious or indefinite character.

After relating several experiments on rabbits, all of which were inflated, and having observed that those which were killed by poison, or which had the spinal cord destroyed, for the purpose of depriving the animal of all sensorial influence, cooled more rapidly than others in which the nervous system was uninjured, he remarks :"The facts now, as well as those formerly adduced, go far towards proving that the temperature of warm-blooded animals is considerably under the influence of the nervous system."

L. For a considerable period, I have devoted my time almost exclusively to one subject of experimental physiology, viz. that of animal heat; and having performed a great number of experiments, modified in almost every possible way, I shall select a few for the examination of those interested in the subject. Before I bring them forward, it is necessary to state that, having an objection to the employment of 
cold air for inflation, I contrived a small machine which enabled me to use warm air, and, at the same time, allowed me to ascertain its temperature whenever I noted that of the animal. It is manifest that the air we inspire is warmed in its passage to the lungs, and, therefore, it is imperative to imitate, as nearly as possible, the operations of nature: As this instrument may be useful to others engaged in the same pursuits, I have given a description of it in the Appendix, to which I refer the reader. The following experiment was performed with cold air. The temperature of the room at the commencement was $43^{\circ}$, and of each rabbit $104^{\circ}$. The colour of the first rabbit was black and white. It was decapitated and inflated. In all the experiments, the rabbits were killed simultaneously, and the different processes attended to at the same moment."

EXPERIMENT I.

$\begin{array}{ccc}\text { Time-Minutes, } & & \text { Thermo, in the Rectum. } \\ 5 & - & 102 \\ 10 & - & 101 \\ 15 & - & 100 \\ 20 & - & 9 \%_{\frac{1}{2}} \\ 25 & - & 96_{\frac{1}{2}} \\ 30 & - & 95 \frac{1}{2} \\ 35 & - & 93 \frac{1}{2} \\ 40 & - & 92 \frac{1}{2} \\ 45 & - & 91_{\frac{1}{2}} \\ 50 & - & 90 \\ 60 & - & 88_{\frac{1}{2}}\end{array}$


The colour of the other rabbit, black and white,-decapitated, but not inflated.

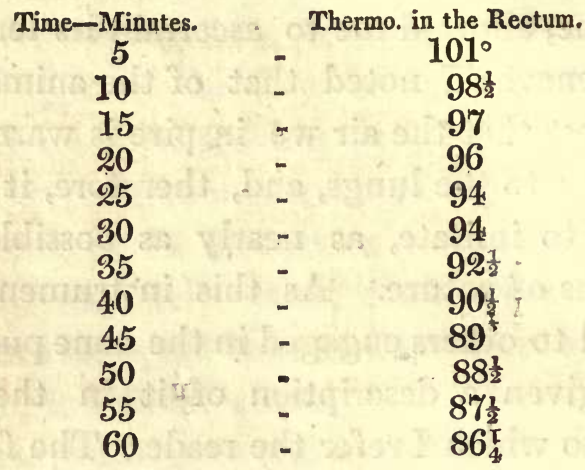

The following was also black and white, but was neither decapitated nor inflated.

Time-Minutes.

5

15

20

25

30

35

40

45

50

55

60
Thermo. in the Rectum. $100 \frac{1}{2}^{\circ}$

99

97

95

93

92

90

89

88

87

86

85

These rabbits were four months old, and apparently equally vigorous. In this experiment we observe, that the one which was decapitated and inflated was at the conclusion $2 \frac{1}{4}^{\circ}$ above the one decapitated but not inflated, and $3 \frac{1}{2}^{\circ}$ above the other, which was neither decapitated nor inflated.

By the kindness of my friends, I was enabled to operate on three or four rabbits at the same 
time, thereby placing these under precisely the same circumstances.

\section{EXPERIMEN'T II.}

Temperature of the room $4 \%$, and of this rabbit, decapitated, and inflated with warm air, 103‥ Five months old.

\begin{tabular}{|c|c|c|}
\hline Time-Minutes & Thermo. in the Rectum. & Temp. of the warm air. \\
\hline 5 & $-102^{\circ}$ & \\
\hline 10 & $103 *$ & 86 \\
\hline 15 & 101 & 86 \\
\hline 20 & $100^{\frac{1}{2}}$ & 85 \\
\hline 25 & 100 & 83 \\
\hline 30 & 99 & 80 \\
\hline 35 & 98 & 84 \\
\hline 40 & 97 & 84 \\
\hline 45 & $96 \frac{\mathrm{I}}{4}$ & 80 \\
\hline 50 & 951 & 86 \\
\hline 55 & $94_{4}^{I^{\prime}}$ & 80 \\
\hline 60 & $94^{4}$ & $\begin{array}{l}- \\
-\quad 82\end{array}$ \\
\hline
\end{tabular}

This rabbit was decapitated, but neither inflated with cold nor warm air. Its temperature $105^{\circ}$; five months old.

$\begin{array}{ccc}\text { Time-Minutes. } & & \text { Thermo. in the } \\ -5 & - & 103^{\frac{1}{2}} \\ 10 & - & 102 \frac{1}{2} \\ 15 & - & 101^{\frac{1}{2}} \\ 15 & - & 100 \\ 20 & - & 98 \frac{1}{2} \\ 25 & - & 97 \\ 30 & - & 96 \frac{1}{4} \\ 35 & - & 95^{\frac{1}{2}} \\ 40 & - & 94 \\ 45 & 93 \frac{1}{2} \\ 50 & - & 93 \\ 55 & - & 92\end{array}$

The following was decapitated and inflated with cold air. Its temperature was $105^{\frac{1}{2}}$; it was 10 weeks old.

* I cannot account for this rise, it is the only case, in the whole series of experiments in which this phenomenon occurred. 
Time-Minutes.

5
10
15
20
25
30
35
40
45
50
55
60

Thermo. in the Rectum.

$$
\begin{aligned}
& 103^{10} \\
& 102 \\
& 99 \frac{1}{2} \\
& 98 \\
& 96 \frac{1}{2} \\
& 95 \\
& 93 \frac{1}{2} \\
& 92 \\
& 91 \\
& 90 \\
& 89 \\
& 88 \frac{1}{2}
\end{aligned}
$$

This rabbit was neither decapitated nor inflated. Its temperature $105^{\circ}$. It was ten weeks old.

$\begin{array}{ccc}\text { Time-Minutes. } & & \text { Thermo. in the R } \\ 5 & - & 991^{\circ} \\ 10 & - & 98 \\ 15 & - & 96 \\ 20 & - & 95 \\ 25 & - & 94 \\ 30 & - & 92 \frac{1}{4} \\ 35 & - & 91 \frac{1}{2} \\ 40 & - & 90 \frac{1}{2} \\ 45 & - & 89 \frac{1}{2} \\ 50 & - & 88 \frac{1}{2} \\ 55 & - & 87 \frac{3}{4} \\ 60 & - & 86 \frac{3}{4}\end{array}$

The four rabbits in the preceding experiment cannot justly be compared with each other, on account of the great difference in age. I have invariably found that animal heat diminishes much more quickly, and toa greater extent, in the young than adult rabbit, and, moreover, that the vigour of the animal seems to retard very much this diminution. The first two may be compared with 
each other. The one decapitated and inflated with warm air, at the conclusion of the experiment, was $3 \frac{1}{2}^{\circ}$ above the other decapitated but not inflated; and we observe the first of the latter two, which was decapitated and inflated with cold air, to be $1_{4}^{10}$ above the one neither decapitated nor inflated. It is unnecessary to bring forward any more experiments in which the rabbit was simply decapitated, as the following will be considered more conclusive and satisfactory, in which the brain was removed, and the spinal cord entirely destroyed.

\section{EXPERIMENT III.}

Temperature of the room $45^{\circ}$, rabbit $105^{\circ}$; inflated with cold air. The colour black and white, and the age four months. Decapitated, and spinal cord suddenly destroyed.

$\begin{array}{ccr}\text { Time-Minutes. } & & \text { Thermo. in the } \\ 10 & - & 103^{\circ} \\ 15 & - & 102 \\ 20 & - & 101 \\ 25 & - & 100 \\ 30 & - & 99 \\ 35 & - & 98 \\ 40 & - & 97 \\ 45 & - & 95 \\ 50 & - & 95 \\ 55 & - & 94_{4}^{3} \\ 60 & - & 93 \frac{1}{4}\end{array}$

The following was not decapitated, but merely inflated with cold air. Its colour black and white, temperature $105^{\circ}$, age four months. 
Time-Minutes.

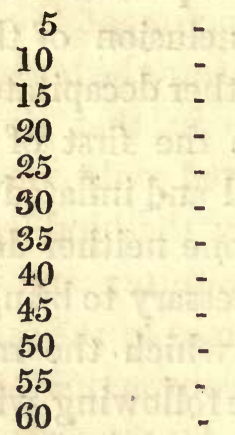

Therno. in the Rectum.

$102^{\circ}$

$100 \frac{1}{2}$

100

$98 \frac{3}{4}$

$97 \frac{1}{2}$

96

$95^{\frac{1}{2}}$

$94^{\frac{1}{2}}$

$93 \frac{1}{4}$

92

92

$90 \frac{1}{4}$

The following was neither decapitated, inflated, nor had it the spinal cord destroyed, as in the first example. Its colour was yellow and white, temperature $105^{\circ}$, age four months.

Time-Minutes.

5
10
15
20
25
30
35
40
45
50
55
60
Thermo. in the Rectum.

$\begin{array}{cc}- & 104^{\circ} \\ - & 103_{4}^{2} \\ - & 102^{2} \\ - & 100^{\frac{1}{4}} \\ - & 99 \\ - & 98 \\ - & 97 \\ - & 96 \\ - & 95 \\ - & 94 \\ - & 92 \frac{1}{2} \\ - & 91 \frac{1}{2}\end{array}$

In this experiment, in which the first rabbit was decapitated, and whose spinal cord was destroyed, its temperature, at the conclusion, was $3 \frac{1}{4}^{\circ}$ above that of the second, which was not decapitated, but inflated with cold air, and $2^{\circ}$ above that of the last, which was not decapitated nor inflated, and whose spinal cord was left uninjured. 
When the chest of the first was examined, at the conclusion of the experiment, the heart contracted 12 times per minute in the horizontal position; allowed to remain in that situation for five minutes, it contracted only once in the minute; when held up by the hind legs, it contracted 20 times in the minute; and when held up by the fore legs, 11 times in the same period. This difference in the contracting of the heart was noticed accidentally by my friend $\mathrm{Mr} \mathrm{H}_{\mathrm{A}}$ milton. The cause cannot be referred to the flow of sensorial or nervous influence, as it possessed neither brain nor spinal cord, but probably to changes in the circulation or determination of blood.

- The chests of the other two were also opened, and the heart of the second was found beating vigorously 75 times per minute; that of the last did not exhibit the least symptom of motion; the heart was dark-coloured with venous blood; its right cavities black, and engorged with the same.

The spinal cord in this, and in all other instances, was destroyed by means of a heated wire, filling as nearly as possible the cavity of the spine. Legallois employed a wire of this size, but Wilson Philip used one much finer, and worked it in various directions, until he conceived the nervous mass to be broken down. We were seldom satisfied with thrusting the wire down from the decapitated surface; the 
spine was often separated at the lumbar region, and the instrument was afterwards sent in a direction opposite to the former.

\section{EXPERIMENT IV.}

The subjects of this experiment were old rabbits. The first was decapitated and inflated with cold air, and had also the spine destroyed, as in the preceding instance. Temperature $106^{\circ}$; colour, black and white.

Time-Minutes.

5

10

15

20

25

30

35

40

45

50

55

60
Thermo, in the Rectum. $103 \frac{1}{2}^{\circ}$

103

102

101

99

98

97

95

94

$93 !$

92

$91 \frac{x}{4}$

This rabbit was merely inflated. Its temperature was $105 \frac{3}{4}$; colour, grey and white.

Time-Minutes,

5

10

15

20

25

30

35

40

45

50

55

60
Thermo. in the Rectum.

$\begin{array}{cc}- & 102 \\ - & 101 \frac{1}{2} \\ - & 100 \\ - & 99 \frac{3}{4} \\ - & 98 \\ - & 96 \frac{1}{2} \\ - & 95 \frac{1}{2} \\ - & 95 \\ - & 94 \\ - & 93 \frac{1}{4} \\ - & 92 \\ - & 91\end{array}$


The following was neither inflated, decapitated, nor had the spinal cord destroyed. Its temperature was $105 \frac{1}{2}^{\circ}$; colour, white.

\begin{tabular}{ccc} 
Time-Minutes. & \multicolumn{2}{c}{ Thermo. in the Rectum. } \\
5 & - & $103^{\circ}$ \\
10 & - & $102 \frac{1}{3}$ \\
15 & - & $101^{\frac{1}{2}}$ \\
20 & - & 101 \\
25 & - & $99_{4}^{3}$ \\
30 & - & $97_{4}^{1}$ \\
35 & - & $97^{4}$ \\
40 & - & $96 \frac{1}{2}$ \\
45 & - & $95 \frac{1}{2}$ \\
50 & - & $94 \frac{3}{4}$ \\
55 & - & 93 \\
60 & - & 92
\end{tabular}

In this experiment we perceive that the first decapitated, and which had also the spinal cord destroyed, lost precisely the same number of degrees as the second, which was inflated without having undergone any mutilation; but we perceive that it suffered a diminution of 3-4ths of a degree more than the one which was laid upon the table to cool.

\section{EXPERIMENT V.}

Temperature of the room $40^{\circ}$; rabbit $105_{4}^{5}$; colour, black and white. In this the brain and spinal cord were removed, as in the preceding instance. 
Time-Minutes.

$\begin{array}{rcc}5 & - & 104_{\frac{1}{2}}^{\circ} \\ 10 & - & 104 \\ 15 & - & 102 \frac{1}{2} \\ 20 & - & 100 \\ 25 & - & 98 \frac{3}{4} \\ 30 & - & 97 \frac{3}{4} \\ 35 & - & 96 \frac{1}{4} \\ 40 & - & 95 \\ 45 & - & 94 \\ 50 & - & 93 \\ 55 & - & 92 \\ 60 & - & 91\end{array}$

Temperature of this $105^{\circ}$; leaden colour. Brain and spinal cord entire. Inflated with cold air.

Time-Minutes.

5

10

15

20

25

30

35

40

45

50

55

60
Thermo. in the Rectum.

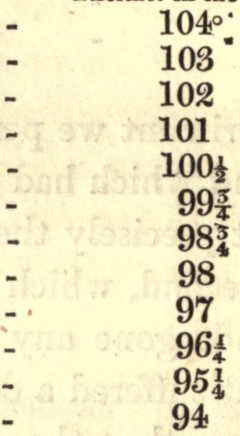

The temperature of the following was $100 \frac{3}{4}^{\circ}$ : colour, grey and white. Laid upon the table to cool.

Time-Minutes.

5

10

15

20

25

30

35

40

45

50

55

60
Thermo. in the Rectum.

$\begin{array}{cc} & 104^{\circ} \\ - & 102 \\ - & 101^{\frac{1}{2}} \\ - & 100_{4}^{\mathrm{x}} \\ - & 99^{\circ} \\ - & 98 \\ - & 96_{4}^{3} \\ - & 96^{3} \\ - & 95 \\ - & 94 \\ - & 93 \\ - & 91_{4}^{3}\end{array}$


This experiment, although it differs in its results from the preceding, yet does not materially, if at all, support the opinion of $\mathrm{Mr}$ Brodie. The rabbit whose head was removed, and whose spinal cord was destroyed, lost $33^{\circ}$ more than the one in which these were entire. In Experiment III. we have an example opposed to this; but, in the present one, the first of the three is only $\frac{3}{4}$ of a degree beneath the last, which suffered no mutilation, nor even loss of blood,-a circumstance that always takes place to a slight extent in the removal of the head and destruction of the spinal marrow. In opening the chests of these rabbits, the heart of the first was found contracting 70 times per minute, while that of the second, in which the nervous system was whole, gave scarcely a perceptible pulsation.

Finding, from a variety of experiments, that it was of little consequence to the evolution of animal heat whether the nervous system were destroyed or left entire, I thought it possible that the results might be different if an animal were killed by some powerful poison. To satisfy myself on thisippoint, the two following experiments were instituted. In the first, inflation was not employed; one rabbit was killed by prussic acid, and the other by a blow upon the occiput; and, thermometers having been introduced into the chest, abdomen, and rectum of each, they were both placed upon the table. The temperature of the 
room $50^{\circ}$-rabbit $101^{\circ}$-colour white,-two months old ; killed by prussic acid.

\section{EXPERIMENT VI.}

Time. Thermo. in the Rectum. Thermo. Abdomen. Thermo. Chest.

$\begin{array}{rllllll}5^{\prime} & - & 99^{\circ} & - & 99^{\circ} & - & 98^{\circ} \\ 10 & - & 97_{\frac{1}{2}} & - & 98 & - & 96 \frac{1}{2} \\ 15 & - & 96 \frac{1}{2} & - & 97 & - & 95 \\ 20 & - & 95 \frac{1}{2} & - & 96 & - & 94 \\ 25 & - & 93 \frac{1}{2} & - & 94 \frac{1}{2} & - & 92 \frac{1}{2} \\ 30 & - & 92 \frac{3}{4} & - & 94 & - & 91 \frac{3}{4} \\ 35 & - & 91 \frac{3}{4} & - & 93 & - & 91 \\ 40 & - & 90 \frac{3}{4} & - & 92 & - & 89 \frac{3}{4} \\ 45 & - & 89 \frac{3}{4} & - & 91 \frac{1}{4} & - & 88^{\frac{3}{4}} \\ 50 & - & 89 & - & 90 \frac{1}{2} & - & 88 \\ 55 & - & 88 & - & 89 \frac{3}{4} & - & 87 \\ 60 & - & 87 & - & 89 & - & 86 \frac{1}{2}\end{array}$

Temperature of the rabbit $104^{\circ}$; colour white; two months old; killed by a blow upon the occiput.

Time. Thermo. in the Rectum. Thermo. Abdomen. Thermo. Chest.

$\begin{array}{ccccccc}5 & - & 102 \frac{1}{2} & - & 102^{\circ} & - & 102 \frac{3}{4} \\ 10 & - & 101^{\frac{1}{2}} & - & 101 & - & 101^{\frac{1}{2}} \\ 15 & - & 99 \frac{3}{4} & - & 99 & - & 99 \frac{3}{4} \\ 20 & - & 99 & - & 98 \frac{1}{2} & - & 98 \frac{3}{4} \\ 25 & - & 97 \frac{3}{4} & - & 97 \frac{3}{4} & - & 97 \\ 30 & - & 96 & - & 95 \frac{1}{4} & - & 96 \\ 35 & - & 95 \frac{3}{4} & - & 96 \frac{1}{2} & - & 95 \\ 40 & - & 94 \frac{3}{4} & - & 95 \frac{1}{4} & - & 94 \\ 45 & - & 94 & - & 95 & - & 92 \frac{3}{4} \\ 50 & - & 93 & - & 93 \frac{1}{4} & - & 92 \\ 55 & - & 92 & - & 93 & - & 91 \\ 60 & - & 91 \frac{1}{4} & - & 92 & - & 90\end{array}$

The temperature of the rabbit killed by prussic acid does not appear to have been peculiarly affected by the poison. 'There is $1 \frac{1}{4}^{\circ}$ of propor- 
tional difference in the rectum; $\frac{x}{2}$ of a degree in the chest; but no difference in the abdomen.

The difference in the indications of the thermometer in these different situations is not to be attributed to variations of temperature, but, probably, as Dr Milligan justly observed to me, is to be referred to the more or less perfect embrace of the contained viscera. In the thorax the lungs collapse when a thermometer is introduced, and thereby prevent the bulb of the instrument from being completely covered,-this is not the case in the abdomen and rectum.

\section{EXPERIMENT VII.}

In this experiment pigeons were substituted for rabbits, and are equally convenient to inflate and operate upon. Temperature of the room $56^{\circ}$ : pigeon $106^{\circ}$; decapitated; spinal cord destroyed ; inflated with cold air.

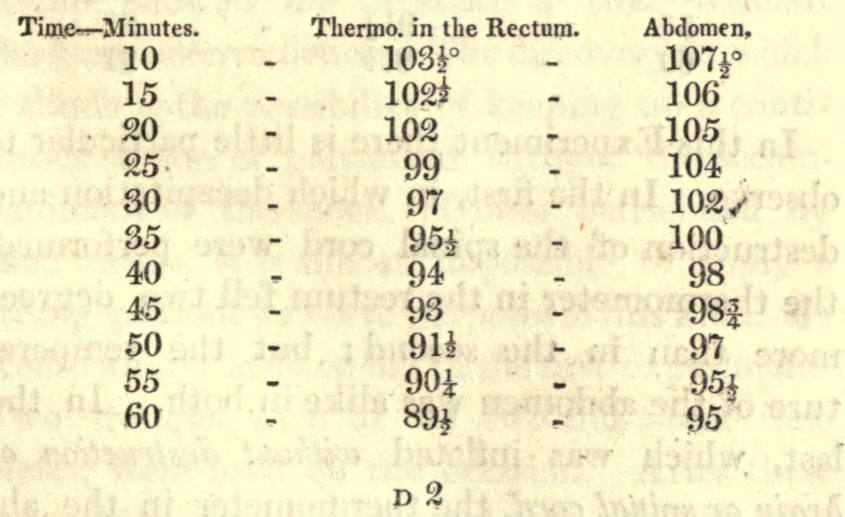


Temperature of the pigeon $106 \frac{35}{4}$; killed by prussic acid.

\begin{tabular}{|c|c|c|c|}
\hline me-Minutes, & Thermo. in the & tum. & Abdomen \\
\hline 10 & $10 \frac{330}{4}$ & - & $105^{\circ}$ \\
\hline 15 & $102 \frac{1}{4}$ & - & $103 \frac{5}{4}$ \\
\hline 20 & 101 & - & 103 \\
\hline 25 & 100 & - & $102 \frac{1}{4}$ \\
\hline 30 & 99 & - & $101^{\frac{\pi}{4}}$ \\
\hline 35 & 98 & - & $100 \frac{1}{2}$ \\
\hline 40 & $96 \frac{1}{2}$ & - & $99 \frac{1}{2}$ \\
\hline 45 & 96 & - & $98_{4}^{3}$ \\
\hline 50 & $94 \frac{1}{4}$ & - & $97_{\frac{1}{4}}^{\frac{3}{4}}$ \\
\hline 55 & $93 \frac{1}{4}$ & - & $96 \frac{1}{2}$ \\
\hline 60 & $92 \frac{i}{4}$ & - & $95_{\frac{3}{4}}^{3}$ \\
\hline
\end{tabular}

Temperature of the pigeon $10 \%^{\circ}$; inflated, without decapitation, or destruction of spinal marrow.

\begin{tabular}{|c|c|c|}
\hline Time-Minutes. & Thermo. in the Rectum. & Thermo. Abdomen \\
\hline 10 & $\begin{array}{l}-106^{\circ} \\
-\quad 103\end{array}$ & $\begin{array}{l}105 \\
103\end{array}$ \\
\hline 15 & $\begin{array}{l}103 \\
1021\end{array}$ & $\begin{array}{l}103 \\
102\end{array}$ \\
\hline 20 & $102^{4}$ & $99 \frac{1}{2}$ \\
\hline 30 & $98 \frac{1}{2}$ & 99 \\
\hline 35 & 97 & $97 \frac{3}{4}$ \\
\hline 40 & 96 & 96 \\
\hline 45 & $94 \frac{3}{4}$ & 95 \\
\hline 50 & 93 & $93 \frac{\mathrm{r}}{4}$ \\
\hline 55 & $91 \frac{3}{4}$ & 92 \\
\hline 60 & $90 \frac{i}{2}$ & 91 \\
\hline
\end{tabular}

In this Experiment there is little particular to observe. In the first, in which decapitation and destruction of the spinal cord were performed, the thermometer in the rectum fell two degrees more than in the second; but the temperature of the abdomen was alike in both. In the last, which was inflated without destruction of brain or spinal cord, the thermometer in the abdomen indicated a temperature of four or five 
degrees less than that of either of the other two, while at the same time the thermometer in the rectum of this had fallen only to the extent of the first in the same situation, and $1^{\frac{3}{4}}$ less than that of the second.

Having, from these and similar experiments, arrived at conclusions unfavourable to those proposed by Mr Brodie, I instituted a variety of others, which seemed to me calculated to strengthen either the opinion entertained by myself, or that adopted by this physiologist. If the generation of animal heat belong rather to the nervous than sanguiferous system, and if galvanism be capable of performing the functions of the former, as imagined by WILson Philip, the employment of this powerful agent ought to be regarded as an additional means to promote the generation of the principle. A valuable discovery of my talented friend, $\mathbf{M r}$ Kemp, allowed me to make a trial without the least inconvenience. The discovery to which I allude is the possibility of keeping up a continuous stream of galvanism without the accompaniment of the shock. Unless befriended by such means, it is almost impossible to apply a strong galvanic power to purposes of this kind. $\mathbf{M r}$ Kemp kindly assisted me in the first experiment. Two troughs, each of 50 two-and-a-half inch plates, were used on the occasion. After these were charged and connected, the positive wire 
was introduced into an aperture beneath the integuments of the spine, and the negative was passed in a circular manner round the neck, previously denuded to ensure the transmission of the galvanism.

\section{EXPERIMENT VIII.}

Temperature of the room $4 \%^{\circ}$; of the rabbit $106^{\circ}$; colour, black and white ; age three months. Without decapitation or destruction of the spinal cord. Inflated with cold air, and galvanism employed for the space of an hour.

$\begin{array}{ccc}\text { Time-Minutes. } & & \text { Thermo. in the R } \\ 5 & - & 104{ }_{\frac{1}{2}} \\ 10 & - & 10 \frac{1}{2}^{\frac{1}{2}} \\ 15 & - & 101 \\ 20 & - & 99 \frac{1}{2} \\ 25 & - & 98 \frac{3}{4} \\ 30 & - & 97 \frac{1}{4} \\ 35 & - & 95 \frac{3}{4} \\ 40 & - & 95 \\ 45 & - & 94 \\ 50 & - & 93 \\ 55 & - & 92 \\ 60 & - & 90 \frac{1}{2}\end{array}$

Temperature of the following, $106 \frac{1}{2} 0$; colour, black ; inflated as the above, without the application of galvanism; age three months.

$\begin{array}{ccc}\text { Time- Minutes. } & & \text { Thermo. in the Ilectum. } \\ 5 & - & 103 \frac{3}{4}^{\circ} \\ 10 & - & 103 \\ 15 & - & 102 \\ 20 & - & 100 \frac{1}{2} \\ 25 & - & 99 \\ 30 & - & 97 \frac{3}{4} \\ 35 & - & 95 \frac{3}{4} \\ 40 & - & 95 \\ 45 & - & 94 \\ 50 & - & 93 \frac{1}{4} \\ 55 & - & 92 \\ 60 & - & 91\end{array}$


Galvanism appears to have had no influence whatever in the present experiment; both rabbits having lost precisely the same number of degrees.

\section{EXPERIMENT IX.}

Temperature of the room $46^{\circ}$; of the rabbit $103 \frac{1}{4}$; colour, grey and white. Inflated with cold air.

$\begin{array}{ccc}\text { Time-Minutes. } & & \text { Thermo. in the } \\ 5 & - & 102 \frac{3}{4}^{\circ} \\ 10 & - & 101^{3} \\ 15 & - & 99^{3} \\ 20 & - & 98 \frac{1}{4} \\ 25 & - & 96 \\ 30 & - & 95 \\ 35 & - & 94 \\ 40 & - & 93 \\ 45 & - & 91 \frac{1}{4} \\ 50 & - & 90 \frac{5}{4} \\ 55 & - & 89 \frac{3}{4} \\ 60 & - & 89\end{array}$

Temperature of the following $103 \frac{3}{4}^{\circ}$; colour, white and black. Inflated with cold air, and galvanism employed.

$\begin{array}{ccc}\text { Time-Minutes. } & & \text { Thermo. in the Rectum. } \\ 5 & - & 102^{9} \\ 10 & - & 101 \\ 15 & - & 99^{\frac{3}{4}} \\ 20 & - & 98 \frac{1}{2} \\ 25 & - & 96 \frac{1}{4} \\ 30 & - & 95 \\ 35 & - & 93 \frac{3}{4} \\ 40 & - & 92 \frac{1}{4} \\ 45 & - & 91 \\ 50 & - & 90 \\ 55 & - & 89 \\ 60 & - & 88\end{array}$


In this Experiment, as in VIII. galvanism does not appear to have produced any marked effect. The rabbit to which it was applied lost $1 \frac{1}{2}^{\circ}$ more than the one in which it was not used. It is necessary to state, that only half of the galvanic power of the preceding experiment was employed at the same moment, viz. one trough of 50 plates. This alteration was made from the fear that it might before have acted too energetically on the nervous system.

Among the preceding experiments there is one in which warm air was employed for inflation. This circumstance will appear strange, after having alluded to the necessity of imitating the process of nature by such means. But having proved, by a variety of experiments, that the warm air, in every case except one, maintained the temperature of the animal from two to three degrees above that of every other, how modified soever the means might be in regard to the latter, I substituted cold air in the place of warm, as it rendered the experiment more easy to the operator, and removed every objection that might afterwards have been brought against the results.

The following experiments are the only two I shall present in which warm air was used. In the first, I was assisted by my friends $\mathrm{Mr}$ Chester and Mr Holroyd. This experiment 
is the one which forms the exception to the above general rule.

\section{EXPERIMENT $\mathrm{X}$.}

Temperature of the room $54^{\circ}$; of the rabbit $101 \frac{10}{4}$.

Time-Minutes. Thermo, in the Rectum. Warm air.

$\begin{array}{lllll}15 & & 99^{\circ} & - & 87^{\circ} \\ 20 & - & 97 \frac{1}{2} & - & 80 \\ 25 & - & 97^{7} & - & 89 \\ 30 & - & 96 \frac{1}{4} & - & 85 \\ 35 & - & 95 \frac{3}{4} & - & 87 \\ 40 & - & 95 & - & 92 \\ 45 & - & 94 \frac{1}{2} & - & 91 \\ 50 & - & 92 \frac{3}{4} & - & 47 \\ 55 & - & 91 \frac{3}{4} & - & 84 \\ 60 & - & 91 \frac{3}{4} & - & 80\end{array}$

Temperature of the following rabbit $1041_{2}^{\circ}$. Inflated with cold air. Both full grown.

Time-Minutes.

15

Thermo. in the Rectum.

$\begin{array}{cc}- & 100^{\circ} \\ - & 100 \\ - & 99 \frac{1}{2} \\ - & 98 \frac{x}{4} \\ - & 97 \frac{1}{2} \\ - & 96 \frac{1}{4} \\ - & 95 \frac{3}{4} \\ - & 95 \\ - & 94 \frac{1}{4} \\ - & 93 \frac{3}{4}\end{array}$

In this experiment, the rabbit inflated with cold air lost 3-4ths of a degree less than the above,-a difference too trifling to note. 


\section{EXPERIMENT XI.}

The result of this experiment may be regarded as affording an impartial view of the influence of warm air in inflation. Temperature of the roam $46^{\circ}$; of the pigeon $110 \frac{1}{2}$. In this experiment I was assisted by my friends Messrs Holroyd and Dunuett.

\begin{tabular}{ccccc} 
Time-Minutes. & \multicolumn{3}{c}{ Thermo. in the Rectum. } & Warm air. \\
10 & - & $108^{\circ}$ & - & $70^{\circ}$ \\
15 & - & 105 & - & 70 \\
20 & - & 103 & - & 71 \\
25 & - & 102 & - & 70 \\
30 & - & 101 & - & 71 \\
35 & - & 100 & - & 70 \\
40 & - & $98 \frac{3}{4}$ & - & 70 \\
45 & - & 98 & - & 68 \\
50 & - & $96 \frac{1}{2}$ & - & 67 \\
55 & - & $95 \frac{x}{2}$ & - & 68 \\
60 & - & $94 \frac{1}{4}$ & - & 67
\end{tabular}

Temperature of the pigeon $111_{2}^{1}$. Inflated with cold air.

Time-Minutes.

10
15
20
25
30
35
40
45
50
55
60

Thermo. in the Rectum.

$109^{\circ}$

$10 \% \frac{1}{\frac{1}{2}}$

$105^{\frac{1}{2}}$

$103 \frac{1}{2}$

102

$100 \frac{1}{2}$

$98 \frac{3}{4}$

98

$95 \frac{1}{2}$

$93 \frac{3}{4}$

$92 \frac{1}{2}$ 
Temperature of the pigeon $111 \frac{5}{4}^{\circ}$ Neither inflated with warm nor with cold air.

$\begin{array}{ccc}\text { Time-Minutes. } & & \text { Thermo. in the } \\ 10 & - & 109_{\frac{1}{2}}^{\circ} \\ 15 & - & 10 \frac{1}{2}^{2} \\ 20 & - & 10 \frac{3}{4}^{3} \\ 25 & - & 103^{-} \\ 30 & - & 101 \\ 35 & - & 100 \\ 40 & - & 98 \\ 45 & - & 97 \frac{1}{4} \\ 50 & - & 95 \frac{1}{2} \\ 55 & - & 94 \\ 60 & - & 93\end{array}$

L. From the results of the preceding and similar experiments, I was persuaded that the removal of the brain or spinal cord had no influence whatever on the apparent developement of animal heat, nor on the degree and velocity of cooling. Still, I could not flatter myself that any of the measures I designed to imitate the natural function of respiration, proved even the slightest increase of temperature. As I did not consider the experiments already detailed sufficiently decisive on this point, I proposed to arrest the circulation of blood in the lungs by tying the heart at its base. The animal being made insensible by a blow upon the occiput, the chest was immediately opened, and the object being accomplished, the divided parietes were again closed, and the inflation of cold air commenced as in other instances. The following experi- 
ments, to elucidate this subject, are not selected, but presented in the order in which they were performed :

\section{EXPERIMENT XII.}

Temperature of the rabbit $105_{\frac{1}{2}}^{\circ} ;$ colour white ; the heart tied at the base; two months old ; inflated with cold air.

$\begin{array}{ccc}\text { Time-Minutes. } & & \text { Thermo. in the Rectum. } \\ 10 & - & 99 \frac{1}{2} \\ 15 & - & 98 \frac{1}{4} \\ 20 & - & 96 \frac{1}{3} \\ 25 & - & 94_{\frac{3}{4}}^{\frac{3}{4}} \\ 30 & - & 93 \frac{1}{3} \\ 35 & - & 92 \frac{1}{2} \\ 40 & - & 90 \\ 45 & - & 88 \frac{3}{4} \\ 50 & - & 87_{\frac{1}{2}}^{\frac{1}{2}} \\ 55 & - & 86 \frac{1}{4} \\ 60 & - & 85^{\frac{1}{2}}\end{array}$

Temperature of the rabbit $104_{2}^{1 \circ}$, colour grey and white; inflated with cold air.

$\begin{array}{ccc}\text { Time-Minutes. } & & \text { Thermo. in the Rectum. } \\ 10 & - & 99 \\ 15 & - & 98 \\ 20 & - & 96 \\ 25 & - & 94 \frac{1}{2} \\ 30 & - & 93 \\ 35 & - & 91 \frac{3}{4} \\ 40 & - & 90 \frac{1}{2} \\ 45 & - & 89 \frac{x}{4} \\ 50 & - & 88 \\ 55 & - & 87 \\ 60 & - & 85\end{array}$


In this experiment, the rabbit in which the heart was tied lost only half a degree more than the other, which was inflated, without having undergone any previous operation.

\section{EXPERIMENT XIII.}

Temperature of the room $48^{\circ}$; of the rabbit $106 \frac{1}{2}^{\circ}$; colour white and grey; full grown. Inflated with cold air.

\begin{tabular}{|c|c|c|}
\hline Time. & Thermo. in the Rectum. & Thermo. in the Abdomen. \\
\hline $15^{\prime}$ & $-102^{\circ}$ & $104^{\circ}$ \\
\hline 20 & 101 & 103 \\
\hline 25 & 100 & 102 \\
\hline 30 & 99 & 102 \\
\hline 35 & $98 \frac{1}{4}$ & 101 \\
\hline 40 & 97 & 100 \\
\hline 45 & $96 \frac{1}{2}$ & $99 \frac{1}{2}$ \\
\hline 50 & $96 \frac{1}{4}$ & 99 \\
\hline 55 & $95 \frac{1}{4}$ & 98 \\
\hline 60 & $94^{\frac{1}{3}}$ & $97 \frac{1}{2}$ \\
\hline
\end{tabular}

Temperature of the rabbit $106^{\circ}$; colour grey and white; heart tied. Inflated with cold air.

Time. Thermo. in the Rectum. Thermo. in the Abdomen.

$\begin{array}{ccccc}15^{\prime} & - & 101^{\circ} & - & 104^{\circ} \\ 20 & - & 100 & - & 103 \\ 25 & - & 99 \frac{1}{2} & - & 102 \frac{2}{4} \\ 30 & - & 98 \frac{1}{2} & - & 102 \\ 35 & - & 67 \frac{1}{2} & - & 101 \frac{1}{2} \\ 40 & - & 97 & - & 101 \frac{1}{4} \\ 45 & - & 96 \frac{1}{4} & - & 101 \\ 50 & - & 96 & - & 100 \\ 55 & - & 95 & - & 99 \\ 60 & - & 94 & - & 98\end{array}$


In this example we perceive that the thermometers in the rectum indicate precisely the same loss of temperature, while those of the abdomen vary $11_{4}^{\circ}$; but this difference is in favour of the rabbit whose general circulation was stopped. The following experiment is the last I shall adduce.

\section{EXPERIMENT XIV.}

Temperature of the room $45^{\circ}$ : of the rabbit $105 \frac{3^{\circ}}{4}$; colour white and grey; full grown; heart tied. Inflated with cold air.

Time. Thermo. in the Rectum. Abdomen. Chest.

$\begin{array}{lllllll}15^{\prime} & - & 100^{\circ} & - & 102^{\circ} & - & 103^{\circ} \\ 20 & - & 100 & - & 1021 & - & 103 \\ 25 & - & 99 & - & 102 & - & 102 \frac{3}{4} \\ 30 & - & 98^{3} & - & 102 & - & 100 \\ 35 & - & 98 & - & 102 & - & 99 \frac{1}{4} \\ 40 & - & 97 \frac{3}{4} & - & 101 & - & 98 \frac{1}{4} \\ 45 & - & 97^{\frac{1}{4}} & - & 101 & - & 98 \\ 50 & - & 97^{-} & - & 101 & - & 98 \\ 55 & - & 96 \frac{x}{4} & - & 100 & - & 96_{4}^{3} \\ 60 & - & 96 & - & 100 & - & 96\end{array}$

Temperature of the rabbit $106^{\circ}$. Inflated with cold air.

Time. Thermo. in the Rectum.

Abdomen. Chest.

$\begin{array}{llclccc}15^{\prime} & - & 102 \frac{3}{4} & - & 105^{0} & - & 104^{\circ} \\ 20 & - & 102 & - & 104 \frac{1}{2} & - & 102 \\ 25 & - & 101 & - & 103 \frac{3}{4} & - & 100 \frac{3}{4} \\ 30 & - & 100 \frac{1}{2} & - & 103 & - & 99 \frac{1}{4} \\ 35 & - & 99 \frac{1}{2} & - & 102 \frac{1}{4} & - & 98 \\ 40 & - & 99 & - & 102 & - & 97 \\ 45 & - & 98 & - & 101 \frac{1}{2} & - & 96 \frac{1}{9} \\ 50 & - & 97 \frac{1}{\frac{1}{2}} & - & 101 & - & 96 \\ 55 & - & 96 \frac{3}{4} & - & 100 & - & 95 \\ 60 & - & 96 & - & 99 \frac{1}{2} & - & 94 \frac{\pi}{2}\end{array}$


The results of this experiment differ little from those already detailed; and from these it appears that animal heat is but slightly, if at all, disengaged in the lungs by any artificial means we possess : but from this statement we are not to conclude that the lungs, in the natural condition of life, are not the seat of any disengagement. I have endeavoured to show, in this and the preceding chapter, that the temperature of an animal is increased or diminished according to the character of respiration or mode of circulation; and I shall now attempt to explain why inflation, however well conducted, does not materially augment the temperature of the body, nor prevent it from cooling in the same ratio as in another unassisted by inflation.

LI. Respiration may be considered partly an instinctive and partly a voluntary function, whose influence is to regulate the circulation of the blood, and therefore the generation of animal heat. If its action be disordered during life, by the preponderance of inspirations, for a short time, the temperature of the body diminishes almost as much as in the rabbit we inflate. The asthmatic patient, of whom Dr BreE speaks, had a temperature as low as $82^{\circ}$ in the paroxysm. I have observed, in Chap. I. xix. and $\mathrm{xx}$. that the air inspired, is not immediately ex= pelled by expiration, but that a portion is emitted-probably the whole which is deteriorated,- 
while a much greater quantity of air at all times exists in the lungs; and from this it is to be inferred, that the successive chemical changes are slow, and of a delicate description. If we send in a quantity of air greater than what is consistent with the laws which nature has established, we destroy the concord connecting the various agents; and this abundance, instead of promoting the different decompositions, tends to destroy the conditions on which these depend.

LII. During the process of inflation, the heart continues to beat; but this is no proof that the circulation is in its normal state, or that the lungs perform their usual office ; for these phenomena occur in the human subject when the temperature of the system has fallen considerably. In fine, the heart occasionally palpitates when the lungs are oppressed with blood; and this symptom is regarded as an increased action of the organ, yet it is often accompanied by a diminution of temperature. It is therefore evident, that the contractions of the heart are far from being criteria of proper chemical changes operating on the lungs.

LIII. The colour of the blood is often adduced as showing that the venous is converted into arterial as in health; but this change of colour takes place in those cases in which respiration is deranged and the temperature diminished. 
LIV. The alterations which the blood undergoes in the lungs are probably various; that of colour is apparent : but this may be only one, and the least of a series, connected with the production of animal heat; or, for any thing that we know, may be an effect altogether independent of those principles which maintain the temperature of the constitution. When venous blood, without the body, is exposed to the action of atmospheric air, it assumes the florid colour of the arterial; but we are not aware that this is accompanied by the generation of heat.

LV. It is stated by Legallois, that he could cool an animal to such a degree, during life, by inflation, as to kill it; and I have not the least doubt that the temperature is, at all times, by such means, materially affected. If we cannot succeed in the application of this process when the animal is alive, and when it in part seconds our intentions, how can we expect to be more fortunate when the animal is mutilated. The combination of gases, and the formation of salts and other substances, are regulated by definite laws; and it is well known, that if proper proportions are wanting, or if those circumstances are absent which are necessary to promote chemical action, no change whatever takes place. But shall we suppose that the powers of life are governed by no principle, or, in more correct terms, that the action of those agents which sup- 
port these, is the effect of chance; if otherwise, let it not be imagined that an imperfect imitation of the function of respiration will furnish results sufficiently accurate to allow us to speak with certainty of its office.

LVI. The cause of animal temperature is attributed by Wilson Philip to a secreting process, similar to that which occasions the variety of secretions in the system by the action of the nervous fluid upon the blood. This opinion is supposed to be demonstrated by direct experiment ; and certainly a great number of facts are brought forward which seem favourable to the view, if such be superficially observed. He has endeavoured to show the close analogy existing between galvanism and the nervous fluid, by numerous experiments on rabbits; and finding that the effects are the same, without considering the complexity of the animal economy, or without looking beyond the mere application of means and the production of obvious results, he concludes that these two agents are precisely of the same nature. The discovery of this seeming analogy has formed an era in physiological science; and if it had been true, it would have been a very close approximation to the developement of the most mysterious operations of the system; but I do not hesitate to say, that while his experiments are to be regarded as furnishing 
facts, his reasoning is to be estimated as merely ingenious and hypothetical.

LVII. Conceiving his opinions on this subject to be incorrect, I shall spare no pains, in the subsequent chapter, to expose errors which are capable of retarding the proper cultivation of medicine for centuries, as presenting to the physiologist established points or data to direct his pursuits, on which, perhaps, he may erect principles not less extensive and important than the present, and thereby would continue to heap error on error, till at last the whole system would fall, from the magniture of its inconsistencies. To do justice to the subject, it is necessary to be minute and patient in the investigation of its merits; and, therefore, I shall follow as closely as possible the observations and experiments of this talented physiologist, for whom, as an individual, I entertain the highest respect. The cause of animal heat will be better understood by the treatment of the following chapter, as I shall have an opportunity of illustrating the importance of respiration and circulation in regard to organic functions.

E. 2 


\section{CHAP. III.}

Secreting surfaces are not dependent on nervous communication between their own individual parts and the origin of the nerves, nor does galvanism re-establish or excite their particular functions by maintaining such a relation.

LVIII. A function may be regarded as the consequence of the inherent properties of an organ, as the power of gravitation is attributed to laws impressed on inert matter; and as the latter is regulated by particular principles, so is the former by the size and activity of those systems essential to its exercise; but the analogy cannot be continued far, from a difference in the principles by which they are governed.

LIX. We observe that the kidneys are supplied with a great quantity of arterial blood, and we still further remark that the urine secreted bears a proportion to this. The relations and organization of the secreting organs would lead one to suppose that the fluid secreted is to be ascribed to an extraordinary mechanism by which the properties of the blood are changed and appropriated, in one situation evolving mucus, in another bile, in a third gastric juice. These products are modified by two causes, disease or derangement in an organ in which the operations are seated, 
disease or derangement of the sanguineous fluid on which the adaptation has to act.

LX. The inflammation of the kidneys is an illustration of the first cause. In this condition its function is almost entirely suppressed; the quantity of urine which is formed is small, and its discharge is accompanied by great pain. Is the explanation of the phenomena to be found in supposing the nerves, or in supposing the delicate and beautiful structure of the parts concerned in the secretion, to be diseased ? What proofs have we that the nerves constitute the mechanism subservient to the function?

In hysteria the urine is pale and copious; at the termination of febrile diseases it is sometimes high coloured, and deposits a thick sediment: In a fit of indigestion how often is it turbid or of an unusual appearance? Are these alterations the effects of a nervous affection, or do they depend on changes induced in the quality of the blood and in its mode of circulation? 'The two latter conditions are obviously disturbed; but we have no evidence whatever that the nerves are equally essential to secretion, or equally disturbed by circumstances.

LXI. In acute cases of dropsy, depletion at times removes quickly the accumulated fluid; and although it is almost impossible to ascertain precisely the conditions of the sanguiferous system which predispose to the effusion, or the 
exact nature of those which tend to diminish it, yet we are sensible that the changes from health to disease, in the present instance, are connected with striking modifications in the circulation of the blood, arising generally from the action of cold, either external or internal, after previous exercise : and we still further remark, that the severity of the symptoms, in every stage of the affection, is characterised by increased force and motion of the blood, the presence of the buffy coat, the increase of temperature, and various other derangements belonging to organic life. If we were to state fully the beneficial changes induced in the same system by bleeding, it would be still more manifest that these depend exclusively, for any thing that we know to the contrary, on the quality of the blood and the mode of circulation. Secretion and absorption in this example bear evident relations to general causes, and to causes which are appreciable.

LXII. The function of digestion has been ascribed by Wilson Philip to the influence of the nerves of the stomach on the materials of the capillary vessels; and, in support of his opinion, he asserts that the secretion of gastric juice is stopped as soon as the eighth pair of nerves are divided, and that this process is re-established as soon as galvanism is transmitted to the organ of digestion. If the appetite be any criterion of the facility with which gastric juice 
is secreted, or of the quantity which is already poured out, it will not be difficult to arrive at certain general laws which regulate the phenomena. Every kind of exercise is a stimulus to the blood; it quickens its motion and improves its properties, and these effects speedily excite those parts of the system appropriated to secretion. The abdominal viscera, without exception, receive the invigorating impulse, indicated by the desire of food and the greater power of assimilation; the colour of the countenance and the temperature of the body, and every natural evacuation, is similarly benefited. But if the body, instead of enjoying gentle exercise, be inactive or subjected to constraint, the above functions become irregular or imperfect; or if an individual be suffering from depressing passions of the mind, the appetite becomes almost immediately fastidious or feeble; and a variety of other symptoms are present, which show that the defect in the desire of food and the energy of digestion is merely one of a series of consequences flowing from the want of oxygenated blood and a regular distribution of it. We have no proof that the nerves in the kidneys secrete, and we have as little evidence that the same agents have that important office in the stomach ; but if we suppose with WiLson PHILIP that these act on the properties of the blood, evolving, in a manner unknown to us, the specific 
fluid, the concession is as dangerous to his reasoning and conclusions as the former unconditional statement; as the quantity and quality of the blood, in all his experiments in which galvanism was not employed, and in which digestion was arrested, were deficient in their most essential conditions.

LXIII. We may state as a principle, that whatever improves the impoverished properties of the blood, or equalizes its distribution, augments the secretion of gastric juice; and that whatever occasions an opposite state of the sanguineous fluid, retards or destroys the function.

LXIV. It is not always easy to ascertain when a function is capable of being performed, or, in other words, what degree of general abberration of the system an organ will bear before it is deprived of its faculty or office. The continuance and the force of circulation enable us in some measure, at times, with precision to calculate the extent of derangement compatible with the exercise of functional power; but the best guide we possess is the temperature of the body. Whenever this is much diminished, or when it falls rapidly, we may rest assured, that digestion will be impeded or destroyed for the time, because the temperature is never low, for even a moderate period, without changing the properties of the blood, and its usual mode of circulation. Having made these observations, we are prepar- 
ed to appreciate the force and correctness of what follows, commencing with the inferences which Wilson Philip supposes to be legitimately drawn from his ingenious and well conducted experiments; and if these be taken in the order presented to the reader, we shall have a better opportunity of judging of the soundness of his views, and of the value of his experiments.

LXV. His first and second inferences, are, "That the function of secretion is destroyed by dividing the nerves of the secreting organs."

"That it may be restored after it is thus destroy$e d$, by the galvanic influence."*

His Experiments, 44 and 45, are adduced to prove the first inference. In $4 \mathbf{4}$, the eighth pair of nerves were divided in one rabbit, in the other they were laid bare, and, after being raised on a probe were replaced uninjured. Both were allowed to eat parsley after the operation. In twenty hours from the division of the nerves, the rabbit which had undergone this operation died, and the one in which the nerves were only laid bare, was immediately killed to allow the experimenter to compare the two. In the latter the food was digested as usual, but in the former the food was found wholly unchanged by the digestive process. Experiment 45 was performed to ascertain whether the distended stomach, which was always a consequence in the rabbit

* Dr Wilson Philip on the Vital Functions, p. 247. 
whose nerves were divided, was to be attributed to the quantity of food which the animal devoured after the operation; and to prove this point, the rabbit was not allowed to eat after the division of the nerves. The stomach was still distended.

LXVI. In these two experiments he does not particularize the symptoms; but he observes, "These experiments seem to leave no room to doubt, that the office of the stomach is suspended by dividing the eighth pair of nerves. A similar observation applies to the lungs. In all the instances in which these nerves were divided, great dyspnoea we have seen come on, and the air cells and tubes were found clogged with frothy mucus."* If an individual suffered from tic doloureux, and allowed the surgeon to divide the nerve of sensation belonging to the face, he would afterwards find that the sensibility of the part was destroyed; and from this operation he would be permitted to conclude that this nerve gave feeling to the face ; but Wilson Philip, in drawing a similar deduction from direct experiment, has apparently forgot two important considerations: First, he ought to have ascertained that digestion depends on nervous influence; Secondly, to have explained the nature of the relation existing between the lungs and stomach in regard to their functions.

* Dr Wirson Philip on the Vital Functions, p. 125. 
LXVII. He grants that the lungs are always disordered, or rather that their office is destroyed, by dividing the eighth pair. Since digestion, in every instance, is modified by the action of the lungs, at one time excited, at another depressed, by the quality of the blood, can we for a moment suppose that the secretion of the gastric juice will take place when the lungs have entirely lost the power of supplying to the stomach, and every other part of the body, a fluid possessing its natural properties? If we believe, with this physiologist, that the nervous energy acts on the blood transmitted to the stomach, it cannot be imagined that the quality and motion of the sanguineous fluid are of trifling consequence to this agent. Does it possess the faculty of improving the deteriorated properties of the blood, of controlling its circulation, and, at the same time, the power of converting it to the necessary wants of the stomach? If such endowments characterized its exercise, the function of the lungs would almost be unessential to the system.

LXVIII. It is stated, that the stomach and osophagus are generally found distended in those rabbits in which the eighth pair of nerves have been divided ; and he says " that this arises from the fruitless efforts to vomit." He does not explain how vomiting distends the stomach, nor can I conceive the possibility, for it is prov- 
ed that this organ is only slightly active in therejection of its contents. If this act were accomplished by the stomach itself, we might then consider it probable that its muscular fibres, by repeated and excessive action, were debilitated, thereby allowing of easy enlargement. The intestines do not become distended in consequence of co. pious evacuations, but have their capacity very much augmented by accumulated fæces or a col$l_{\text {ection }}$ of air. The bladder, if ever found in a similar condition, has not become so from the approximation of its parietes from a pressure from without, which occurs in vomiting, owing to the action of the abdominal muscles, but from the influence of a distending force from within. The cause of this phenomenon is to be ascribed to the disengagement of aëriform fluids, which generally happens when digestion is imperfect, or when it is almost destroyed. The food which the stomach receives is not acted upon in the ordinary way, but the action of the gastric juice in the organ is sufficiently powerful to make an attempt at decomposition; and this imperfect action is attended by an evolution of aëriform fluids. From the developement of this view, it is clear that the stomach, like the bladder and intestines, is distended from a power exercising a pressure from within, outwards, not from efforts of vomiting, which are accompanied by opposite physical circumstances. 
LXIX. We now come to the consideration of the second inference,- "that it (secretion) may be restored, after it is thus destroyed, by the galvanic influence." Experiments 46, 47, 48, and 49 , were performed for the purpose of shewing that the influence of galvanism was equivalent to that of the nerves. In the first; 46, in which the eighth pair of nerves were divided and galvanism applied, neither dyspnoea nor vomiting occurred:-these invariably happened in every instance in which the lungs were found congested with mucus, and covered over with black patches; consequently, their absence shews that the circulation of the blood in the pulmonary tissue was little altered ; and, moreover, that its properties were but slightly modified. Such conditions being maintained, digestion ought to proceed in the usual way; and this was found to be the case. The animal lived six hours, and on examining the lungs the following were the appearances: "The membrane of the trachea was of a natural colour, and there wasno fluid in it. The ramifications of the bronchia in the left lung were quite free from frothy mucus. There was some fluid in the right lung, though it did not appear much gorged; there was one dark spot on it. The lungs collapsed imperfectly on opening the chest."*

Case 47. is very interesting, as it more strik-

* Dr Wrison Phirrp on the Vital Functions, p. 125. 
ingly illustrates my opinion than almost any other which he adduces. Two full grown rabbits ate plentifully of parsley half an hour before the division of the eighth pair of nerves. As soon as this operation was performed on one, galvanism was applied, and continued to be so, with varying degrees of intensity, for seventeen hours, at which time the rabbit died; the other, which had not been in any manner interfered with, was then killed, and the two were compared. He says, "The only differences between the contents of the two stomachs were the following:-The food, which the healthy rabbit had taken during the experiment, was found in the cardiac portion of the stomach, and digestion was going on rapidly in it; while that which the other had taken at the same time was still in the oesophagus, and consequently unchanged." It appears that the food which had been taken previously to the experiment exhibited in both an equal extent of the digestive process; and this is consistent with the state of respiration during that time. After galvanism had been employed twelve hours, he says, "The animal seemed very composed, and breathed with considerable freedom, the trough having acted regularly for some hours." * Two hours after this period the animal made an attempt to vomit, and from this time the breathing became labo-

* Dr Wrlson Prilir on the Vital Functions, p. 130. 
rious, and the severity of this symptom continued to increase till death.

While the lungs were enabled to act with regularity, the stomach was scarcely, if at all, impeded in its functions; and this accounts for the similarity in the results presented by the food which had been takenbefore the operation, and the irregularity or imperfection in the respiration after the animal had been enfeebled or oppressed by the operation. Application of galvanism, and the constraint of its position, accounts also for the food being in the œesophagus, and consequently unchanged, while that which the other had taken was in the cardia, and in part digested : so that the latter part of this experiment differs little from others which he offers to our notice, in which the nerves were divided without being followed by the employment of galvanism. In accordance with this opinion is the condition of the lungs as described by Wilson Philip: "The lungs did not collapse on opening the thorax, the air-cells being full of a frothy and bloody serum. The lungs were externally of an uniform dark-red colour. The heart was a little increased in size, and highly vascular."*

LXX. Experiment 49. on two dogs, presents nothing of importance, except that the one in which

* Dr Wilson Philip on the Vital Functions, p. 132. 
the eighth pair of nerves were divided, and in which case galvanism was not used, had great dysp. noea and made violent efforts to vomit in ten minutes after the operation; and in four hours, at which time it was killed, "it was so weak that it could not stand or move itself from the place where it lay on its side." * And he farther observes, "Is it possible to explain the results of these experiments without admitting the identity of the nervous influence and galvanism? We must either admit this, or that there is another power capable of performing the most characteristic and complicated functions of the nervous system." + Having already made many observations on the character and results of these experiments, it is scarcely necessary to discuss at length the evident incorrectness of the inferences which he deduces.

LXXI. Without supposing any identity between nervous and galvanic influence, the results are easily explained; nor are we necessitated, if we deny this identity, to admit, that there is another power, "capable of performing the most characteristic and complicated functions of the nervous system." The views already developed, in conjunction with others to be stated, will preserve us from this disagreeable dilemma.

* Dr Wrison Phurip on the Vital Functions, p. 136. $+I b i d$. p. 138. 
LXXII. Galvanism is one of the most powerful stimulants we possess : it is capable of discussing indolent tumours, of exciting the action of the heart, and of improving the sluggish state of the circulation. I am acquainted with a gentleman who has employed galvanism in two syphilitic buboes of the groin. One was about the size of a hen's egg, and this, after half an hour's application of a small pile of forty two-inch plates, was diminished onehalf; in the other, which was less, very slight alteration immediately took place in its size or appearance, but in this case resolution quickly followed its employment.

LXXIII. The action of galvanism, in WILson Philip's experiments, is similar to the operation of the same power on any external tumour. When transmitted to the lungs and stomach by the ends of the divided nerves, it causes the blood to circulate with greater facility and regularity. In proof of this I may observe, that, when its influence is discontinued for a very short period, congestion immediately takes place in the lungs, indicated by laborious breathing and vomiting, but these symptoms as soon disappear when it is reapplied; and, still further, it is acknowledged by this able physiologist, that great vascularity was generally found in those cases in which galvanic influence had been employed. If, then, this agent diminishes congestion, and 
promotes a more equable circulation in the lungs, the blood will be better oxygenated according to principles previously stated, and, therefore, as the state of the stomach depends on these conditions, its function will be accelerated according to the correctness with which they are performed. Galvanism will also act upon the stomach in the same manner that it does upon the lungs; but the effects will here be confined to an improvement in the distribution of the blood, while, in the other case, it tends to improve the properties of this fluid by removing all disabilities to its circulation, and to the chemical influence of the inspired air.

If I were required to bring forward any proofs in support of the influence of galvanism, 1 would adduce the application of this agent to practice, and the results which followed its action, as stated by Wilson Philip himself. He says, " That both the oppressed breathing and the collection of phlegm caused by the division of the eighth pair of nerves, may be prevented by sending a stream of galvanism through the lungs :" " And still further, " When there is a considerable tendency to inflammation in habitual asthma, the repeated application of galvanism sometimes increases it so much, that the use of this influence no longer gives relief till the in-

- Dr Wilson Phunir on the Vital Functions, p. 315. 
flammatory tendency is subdued by local bloodletting." * It was found most beneficial in those cases " which were least complicated with other diseases, the chief complaint being a sense of tightness across the region of the stomach, im. peding the breathing."

Are the oppressed breathing and collection of phlegm prevented by galvanism acting in a peculiar manner upon the nerves, or in removing the physical causes which produce these symptoms? Do oppressed breathing and collection of phlegm arise from an affection of the nerves, or from $a$ condition of the system incompatible with the free circulation and oxygenation of the blood? The. reason that galvanism is injurious in those cases of habitual asthma characterised by a tendency to inflammation, is, that inflammation is itself an over-excited state of the capillary vessels of the affected part; and if we apply this agent, it excites still more, according to its universal influence, and therefore will augment the disease, It is beneficial in cases of congestion, because it stimulates the vessels engorged, and enables them to overcome the burden by which they are oppressed.

LXXIV. This explanation does not prove the identity of the nervous and galvanic power, because the office of the eighth pair of nerves is

* Ibid. 325. 
not to operate upon the blood in the same way as the aura which is furnished by the pile, but to regulate the respiratory functions, whose business it is to exercise a more extended influence upon the qualities of the sanguineous fluid and its mode of circulation; and as long as these circumstances are attended to, whether by the normal action of the nerves, or the stimulus of galvanism, the stomach will continue to secrete gastric juice; nor does the explanation compel us to look out for some other mysterious agent whereby we shall be enabled to give reasons, why these apparently complicated functions are performed during the absence of their proper instruments. We shall now pass to another inference, No. 24.; and after critically examining its merits, we shall be better prepared to judge of the correctness of those principles promulgated by this physiologist, and the reader will then perceive more clearly the value of others which are substituted in their place:

"That lessening the extent of the nervous system, by destroying the influence of any considerable part either of the brain or spinal marrow, deranges the secreting power." *

LXXV. Wrlson Philip having proved that digestion is destroyed when the eighth pair of nerves are divided, was desirous of ascertaining

* Dr Wilson Philip on the Vital Functions, 247. 
whether the same effect would follow the partial destruction of the spine, as he believed the stomach to receive nerves from this as well as from the brain. To place the question beyond doubt the following experiments were performed, -58 , 59, 60, 61, 62. As 58. contains few particulars, we shall pass to the next.

In a full grown rabbit a small wire was introduced into the spine at the fourth lumbar vertebra, by which the spinal marrow was destroyed as far as the lowest dorsal vertebra. "Respiration was a little disordered. In a short time after the operation, the animal appeared lively, and ate some parsley. The respiration continued to be slightly affected. Some hours after the operation, Mr Hastings, who watched the animal, observed it to be very cold, and it shivered, although it was kept in the same temperature with other rabbits, who shewed no signs of being cold. The rabbit used in the last experiment, 58 , also seemed cold, but not in the same degree. The respiration now seemed much disordered, and the animal refused parsley. It died in 27 hours after the operation."* After death the following appearances presented themselves:- "The stomach was not much distended. The parsley near the cardiac orifice was not at all changed, and that near the pyloric orifice very slightly. The membrane of the trachea and bronchia was

* lbid. p. 172. 
more vascular than natural. The bronchial cells were slightly loaded with frothy mucus, and there were the same red patches in the lungs as after dividing the eighth pair of nerves." From bringing forward the symptoms as described by W-Ilson Philip, it is scarcely necessary to make any observations upon them: they differ little from those which have been already detailed, and the results are of the same kind.

LXXVI. Disordered respiration is one of the first symptoms which he mentions, and this continued for many hours. The animal was lastly observed to be very cold and shivered, which sign has been stated in Lxiv. of thepresent Chapter to be the most sure indication of a condition of the system incompatible with the essential functions of life. The cause of the cold which it experienced is found in the description which is given, " the bronchial cells were slightly loaded with frothy and bloody mucus." Experiment 60. will illustrate more accurately the precise states of the temperature. In this experiment he wished to try the effects of the destruction of a smaller portion of the spinal marrow, and, for this purpose, introduced a small wire into the spinal canal, at the first lumbar vertebra, "and that part of the spinal marrow which lies below this vertebra was destroyed." The state of the respiration is not mentioned, and from this, one is led to suppose that there was nothing peculiar about it. "On 
examining it after death, the stomach was found no larger than natural ; the parsley retained its colour, smell, and fibrous texture, although such a change had taken place in it as demonstruted a very slight degree of the digestive process."

It is to be regretted that few particulars are given concerning this rabbit. We are told that in 35 hours after the operation the animal died; but the cause of this is not stated. The most important contribution in the experiments at which we have glanced is a table, in the present one, of the temperature of the animal at different times. From inspecting this, and attending to the observations which accompany it, I cannot but flatter myself that the views which $I$ have endeavoured to establish in this and the preceding chapters, receive a more correct corroboration than they possibly can from reasoning founded on general facts. Its importance precludes the necessity of apologizing for its insertion.

"The bulb of Fahrenheit's thermometer introduced into the mouth, and kept there for two minutes previous to the experiment, stood at $98^{\circ}$.

Immediately after the operation, Therm. - $\quad 98^{\circ}$

In twelve minutes after it, _ _ _ _ $\quad 92$

In half an hour after it, _...

In two hours and a half after it, - $\quad$ - 98

In five hours and three quarters after it, $\quad-\quad 98$

In seven hours and a quarter after it, _ _ 98

In nine hours after it, _ $\quad$ - $\quad 96$

In ten hours after it, $\quad-\quad+\quad-95$ 
The animal during all this time appeared lively, and ate parsley.

In eleven after it,

In twelve hours,

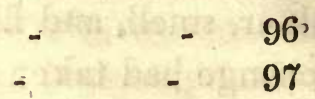

Night coming on, the temperature was not measured for thirteen hours. In the morning the rabbit appeared lively, and ate readily.

In twenty five hours after the operation, $\quad-\quad 88$

In twenty-seven hours after it, _ _ _ $\quad 84$

In twenty-nine hours after it, $\quad-\quad-88$

In thirty hours after it, $\quad \ldots \quad \ldots \quad 84$

In thirty-one hours after it, _ _ $\quad-\quad 84$

In thirty-three hours after it, - $\quad$ - 80

The animal still continued to eat. In thirty-four hours after the operation the temperature was $75^{\circ}$."

"This animal did not appear nearly so cold as that in the preceding experiment, in which a larger and more important part of the spinal marrow was destroyed."

It is difficult to conceive how so acute a physiologist as Wilson Philip could watch the diminution of temperature, the laborious breathing, nay, even the shiverings of the animal, and yet keep his attention fixed exclusively upon the stomach and the nerves. 'This fact ought to make us extremely lenient towards those less gifted, and possessing fewer opportunities of observation. When the temperature of the human constitution is raised or depressed a few degrees, the se-

* Dr Wrlson Philip on the Vital Functions, p. 176. 
cretory organs are invariably deranged, at times almost suppressed, and although there may be a difference in the delicacy of organic functions in the higher and lower gradations of the animal creation, yet, when we perceive the temperature of a being, perhaps not less sensibly constituted than ourselves, from 15 to 23 degrees beneath its natural standard, have we a right, as observers of cause and effect, to anticipate ordinary consequences? In the whole class of hibernating animals, whose peculiarity it is to dose away a third or fourth part of their existence at a period of the year when their necessities could not be supplied, the feelings which prompt them to look out for food weaken with the diminution of their temperature; and as these feelings, in health, are proportionate to the activity of internal functions, it is manifest that the influence of cold, whether arising from external or internal conditions, has a regularity in its action, and a uniformity in its effects. The nature of my experiments compelled me to keep a great number of rabbits, and the necessity of passing many hours a-day among them, readily disposed me to investigate their habits, and to consider the agency of general causes upon their economy or system, such as heat, cold, and perturbations of the mind. Whenever the heat was increased by a change in the seasons, or by additional warmth in the room, the animals became more lively, 
their appearance in the space of a week or a fortnight more vigorous and sleek-but, as soon as the thermometer, in the situation in which they were placed, was as low as $\mathbf{3 5}$ or $\mathbf{4 0}$ degrees, they presented marked alterations. They crept along as if afraid to expose a large surface of the body to the cold, the sleekness - vanished, and the spine soon shewed an unusual prominence, and, when these signs were evident a nightly mortality lessened the stock. For some time I attributed their haggard and consumptive aspect to disease; but after reflecting on the influence of cold in modifying the qualities of the blood and its distribution, I was naturally led to regard the assimilative powers of the system as affected by these changes; and, believing this opinion to be correct, I endeavoured to obviate former consequences by the application of principles deduced from the consideration of their cause. When the days in winter were particularly severe, I placed the young and sickly in a large box kept in the room with the others; and, by pursuing this plan, I preserved their number and increased their vigour. It is scarcely possible to convey an idea of the difference I perceived, in point of liveliness and health, between those subject to a temperature of $75^{\circ}$ in a confined place, and those exposed to $35^{\circ}$ or $40^{\circ}$ in a small room.

I have not made any experiments on the most 
sickly rabbits, for the purpose of ascertaining the variations of temperature from the state of health; but among the great number which I have examined, in which some were strong, and others weakly, I have seldom found a difference beyond $5^{\circ}$ or $6^{\circ}$; but if this diminution of temperature produces such marked results, can we expect a rabbit to digest its food when constrained and tortured by the experimenter, with laborious and irregular breathing, and a loss of $15^{\circ}$ or $20^{\circ}$ of animal heat? If this fact can be established, the secretion of gastric juice is regulated by a partial or individual law - a law which is independent of those great principles of the system, and of every modification which these suffer from internal or external general causes.

The last inference and experiment which I shall at present notice, are the following - "That dividing the spinal marrow does not derange the secreting porver." To prove this, the spinal marrow was divided about the middle. "The rabbit seemed lively after the operation, and continued to eat frequently till within six hours of its death. It died in twenty-seven hours and a half after the division of the spinal marrow. It had not vomited, and had little or no dyspnoea."* In this experiment the digestive power was little, if at all, affected ; and this is just what we should anticipate from the symptoms which he describes,

* Dr Wilson Philip on the Vital Eunctions, p. 180. 
as these show that the respiratory function was scarcely at all troubled. He remarks, "It had not vomited, and had had little or no dyspnoea. The lungs collapsed on opening the thorax, but contained only a little frothy mucus." If the reader will compare this description with those previously given, he will acknowledge the present experiment to be corroborative of the opinion continually expressed concerning the cause of the cessa. tion of digestion. Wilson Philip, in speaking of the dark red patches, which always characterised the lungs of those rabbits in which the eighth pair of nerves had been divided, observes, that he cannot compare these patches to any thing except what "now and then appears in the lungs of the entire animal after it has lain dead for many hours. In the living animal it was always proportioned to the degree in which the secreting power of the lungs was deranged, appearing to the greatest degree when the congestion of the lungs was greatest; and not appearing at all, although the eighth pair of nerves had been divided, when the breathing was rendered free, and the congestion prevented, by galvanism."*

LXXVII. Many fallacies in reasoning are concealed by an ingenious or sparing application of principles. The view presented seems correct, because one phase is alone offered, and this of a

*. Dr Wilson Philir on the Vital Functions, p. 216. 
specious description; but when the application of these principles becomes general, or when it is pushed to the extreme, the variety and prominence of objects immediately expose their inefficiency or incorrectness; and I regret to say, that the principles which we have examined in this chapter, are an illustration of the opinion.

LXXVIII. Secretion, agreeably to the doctrine of Wilson Philip, depends on the operation of the nervous power. "To this (he says) it may be objected, that plants and the less perfect animals have no nervous system. Would it not be more correct to say, that the operation of their nervous system is more confined? Wherever secretion is performed, the nervous influence, or a power resembling it, must exist."*

That the phenomena belonging to secretion are attributable to nervous influence, or a power resembling it, is a gratuitous assumption. It has never been proved that plants are endowed with nerves; and yet we perceive that they are capable of affecting the air in much the same manner as animals; they have an ascending and descending circulation; they have radicles which select and absorb what is beneficial to their growth ; and the leaves which they possess operate as lungs on the materials conveyed; and these, after proper elaboration, are appropriated to the yarious systems of the vegetable, which, in their

Ibid.p. 258. 
turn, are exercised for the growth and support of organic life.

LXXIX. In those beings which constitute the links between animal and vegetable existence, no nervous system has been detected; and yet, from the more complex actions which these exhibit, it is reasonable to suppose, if such, or similar actions, in a more perfect organization, are invariably connected with or produced by nerves, that a nervous system ought to exist. The ab. sence of such parts ought to teach the physiologist to investigate minutely the systems of those individuals who exhibit only the first rudiments of vegetable and animal life; and if these have an operation or design analogous to the systems of those whose functions are more extensive, whose relations are more numerous, and whose susceptibilities are greater, he must not measure the laws of the former by his indefinite ideas or principles derived from the latter. If he does, the consequence will be, that the systems of the former will remain unknown, and his knowledge of those of the latter will be confused or uncertain.

Abbé HAUY, by taking a crystal, and chipping it, was enabled to discriminate the form of its original nucleus or constitution; but to detect the important laws of the animal creation, we must not commence by removing the many additions which have been bestowed and multipli- 
ed for the adaptations, purposes, and circumstances of life, but must commence where the crystallographist terminates; and, by attending to this plan, we shall unravel the principles of more perfect systems, which are concealed by parts of secondary formation or importance, scarcely at all essential to the exercise of these principles.

LXXX. As it may justly be asserted that our senses do not allow us to penetrate deeply into the structure of organized beings, and, therefore, we have no right to deny what we cannot demonstrate, we will leave plants and imperfect animals, and examine the merits of the doctrine in a manner altogether unexceptionable.

LXXXI. A few cases are on record in which foetuses were born without brain or spinal marrow ; and yet these possessed external endowments characteristic of the activity of internal functions; indeed there was no observable difference between such and others in whom these organs are entire.

LXXXII. The difficulty which this case presents being insurmountable according to the principles laid down by this physiologist,-he says, "No writer, as far as I know, has attempted to explain the difficulty." He confesses that his experiments do not explain it; but he proposes the following view : "If the nervous system be galvanism, there may be some apparatus in the uterine system for collecting and applying 
this agent, which is every where diffused, till the brain and spinal marrow can perform their functions, and which may continue to supply their place where they never exist."* Throughout this chapter I have endeavoured to show that it is to alterations in the mode of circulation, or in the qualities of the blood, that the different phe. nomena of the digestive process must be attributed; and, still farther, that all secretions depend on the sanguiferous system for the extent and activity of their functions :- and this reasoning, although opposed to the generally received principles, was found to be supported by the disorders induced in the respiration and circulation, and in the evident disease or derangement of the lungs, in all the experiments brought forward.

LXXXIII. The division which Bichat has supported of animal and organic life does not apply strictly to the foetus. It does not require a system of nerves to elevate or depress the ribs, to give motion and sensibility to the body, so that it may be locomotive or taught by its sensations to preserve itself; and as the senses are shut from the external world, the brain is unessential to its present situation. It, therefore, possesses chiefly organic life; and at this period, although complex and varied in its organization, it is almost as uncompounded as a vegetable, and regulated by nearly similar laws. The difference between the foetus

* Dr Wrison Phritr on the Vital Functions, p. 241. 
and new-born infant is great. The latter is bringing into play, by the exercise of its limbs, and the stimulus of external objects, the endowments of the nervous system, while the former has no necessity for them. But there is no difference whatever between the foetus and the infant in regard to those systems by which an animal is nourished. One is supplied with arterial blood from its mother, and this is distributed to every part of the body ; the vital principles of which it is composed are appropriated to the increase of the embryo parts precisely in the same manner as when these parts are subsequently developed and maintained by blood elaborated by its own organs. The nervous system is as little related in after life as at that time to those peculiar adaptations by which one general fluid is converted into the varieties which the constitution exhibits. It will produce modifications, because it supplies sensibility and motion, whose influence is extensive and multiplex.

LXXXIV. It is therefore obvious, that the difficulty, of which WILson PHILIP speaks, is a difficulty only according to his own principles. The solution which is here given is not derived from hypothetical sources, but is furnished by the same reasoning and observations which have enabled us to expose evident inaccuracies in the views which he has developed, as explanatory of the process of digestion and the generation of animal heat. 


\section{CHAP. IV.}

\section{The Distribution of the Blood at different Ages and Seasons.}

LXXXV. Wr have been long accustomed to regard the circulation of the blood as composed of an arterial and of a venous system, continually in action; but our attention has never yet been sufficiently awakened to investigate the important alterations of which these systems are susceptible. The lungs and the heart commence their functions at birth, and as long as life continues they perform the designs of Nature; but these designs and effects are variable, because the necessities of the system change with the evolution of new, or the feebleness and imperfection of old organs. The lungs unceasingly act upon the blood, and the heart every moment conspires to propel it; but the former do not always possess the same capability, nor does the latter exhibit the same energy through the different stages of existence. If then the qualities of the blood are modified by the different conditions of organization, and if its propulsion and circulation are also subject to the same, is it philosophical to suppose that its distribution will be equable or uniform? 
LXXXVI. In physics an effect is proportionate to its cause, and the same holds good in the animal frame in regard to all actions that are referrible to this department of science. If a ball be thrown from the hand with great force, it will overcome many obstacles which would retard the course of another propelled with less energy; but suppose this ball to be thrown through a given space where there are different objects presenting different degrees of attraction or controlling pozoers, and then we have an idea of the office of the heart and the influence of certain organs in regulating or restraining the diffusion of the sanguineous fluid.

LXXXVII. Physiologists and Anatomists have long heen occupied with the minute structure of the heart and lungs, endeavouring to unfold their ultimate tissues, to describe the courses or uses of vessels, or the number of nerves which enter into their composition; and these investigations have been productive of many theories and fanciful opinions, which, if true, would have led to no application, but have given rise to one consequence, which has perhaps tended more than all others to mystify the operations of $\mathrm{Na-}$ ture,-I allude to the study of minute objects. While the mind and the eye have been equally on the stretch to understand and perceive the nature of ultimate principles, they have almost entirely forgot to observe and examine phenomena 
which are more obvious in their character, and more easily traced. In the attempt to explain the difficulties of the present subject, which, as yet, has never been investigated, I may probably fail in convincing all of the correctness of the views; but it cannot be denied that the application is practical.

LXXXVIII. In the first months of infantile life, the respirations are extremely numerous, presenting from 30 to 50 inspirations in a minute; the heart is also proportionately quick and frequent in its pulsation; and the heat of the body is generally above the standard of the adult.

LXXXIX. The nourishment of the infant is derived from the breasts of the mother, and this is of a rich and nutritious quality, well adapted to support and extend the powers of life, without requiring much elaboration on the part of its organs to fit it for the purposes of nature. The digestive process, which brings into action the greater part of the abdominal viscera, is, therefore, on the present occasion, not so energetically exercised as to make great demands upon the blood.

And if it be acknowledged that the assimilating organs are less powerfully exercised than in after life, the brain, and those parts of the system subsequently developed for the procreation of the species, are to be considered as influencing even less the mode of circulation or the expenditure of the blood. Although the former 
is more perfectly formed at this period than the latter, because more early brought into requisition, yet it is manifest, that, for some time after birth, its action is too feeble to constitute an expensive function of the system.

XC. It may probably be advanced against the present argument, that the rapid progress of the infant, evinced in the expansion of the body, demonstrates that the functions appropriated to organic life are more active than those of the adult. This idea seems plausible at the first glance; but if it be remembered that the child is subject to little or no exertion; that its waking and sleeping moments are unruffled by real or imaginary evils; that its digestive powers are not deranged by the improprieties of habit, or the influence of moral causes; and, lastly, that its existence may be said to be distributed into three parts, two of which are devoted to sleep, or a state equivalent to it, and the other to the breast,-it will then appear obvious, that this effect does not require superior internal energy on the part of the assimilating organs to give an efficient reason for its occurrence.

XCI. The embonpoint of the system in adults depends less on the quantity or quality of food than is generally imagined. Rest, peace of mind, and a moderate indulgence of our passions, are the principal agents which contribute to pros duce this result. Indeed the more nearly we 
approximate to the life of the infant, the more closely will our frame resemble the form and softness of the infantile system.

XCII. The frequent respiration of the infant explains the quick and numerous contractions of the heart; and the existence of these conditions, the internal organs being comparatively inactive, proves that the blood must be more generally and equally distributed to every part of the system. At this age, the circulation, in contradistinction to what subsequently occurs, may be called external; and, from views which I intend in the next chapter to unfold, it will be obvious, that this character of the circulation belongs to the young of every kind; and, still further, the knowledge of this peculiarity will teach us to estimate the influence of external causes in augmenting or diminishing the generation of animal heat.

XCIII. As soon as the infant ceases to be dependent on the mother for its support, the digestive functions acquire a greater energy ; and the consequence of this change is, that the stomach, liver, and the whole of the intestines, as well as the excretory organs, make a continual and more extensive demand upon the properties of the blood, and necessarily must modify the previous circulation of this fluid, as these internal actions must be regarded in the light of attractive or controlling powers. In accordance 
with what is here advanced, it is observed, that the respiration is less frequent, and the contractions of the heart less numerous, as we proceed from infancy to maturity; and the power of bearing cold is augmented in extent proportionate to these changes, not because the power of generating heat is increased, as supposed by Dr EDwards of Paris, but because the agency of external causes have not the same states of the circulatory system to influence,

XCIV. In tracing the infant a little further in its progress; we perceive the gradual development of animal life, and the more vigorous exercise of those parts belonging to the organic. The mind is not only susceptible to impressions, but is anxious to cultivate a knowledge of the various objects by which it is surrounded; and the different sports of this age are calculated to give strength and agility to the body, acuteness to the senses, and vigour to the assimilating functions.

$\mathrm{XCV}$. In harmony with what I stated in xc. and xcr. the child loses, as it approaches the teens, that chubby fulness which characterised its earlier years, shewing that this con dition was not to be referred to the activity of the digestive process, but to the sluggishness of mental and physical powers which are subsequently brought into action. Without dwell, ing any longer on gradations of the system, that 
will not be fully appreciated by all, we will at once commence to treat of a period of life which developes the distinctive characters of sex.

XCVI. I have already pointed out in what manner the internal or vital organs of the system influence the circulation of the blood, viz. by their increased importance and energy; and when these have, for some time, given a different inclination to the distribution of this fluid, the various organs appropriated to the animal propensities appear as additional stimuli or powers to complete that inclination.

XCVII. The development of these parts, in relation to the blood which must supply them, cannot be considered in the light of a simple increase of matter requiring nerves and bloodvessels for their nourishment, but must be regarded as bringing into play new and essential functions, endowed with principles independent of the general laws of the system, except in so far as these contribute to their wants.

XCVIII. All secretions of the system are accomplished by certain organs or adaptations, in a way unknown to us, but at all times at the expence of the sanguineous fluid, and they always bear a ratio to the quantity they receive in the state of health. It is therefore evident, that, if we augment the secretions, and particularly such as are not intended for the support of the constitution, we must diminish the proportions previ- 
ously distributed to the different organs, and these proportions will be disturbed according to the exercise or energy of the individual functions in question.

XCIX. The organs that are late of being developed, when perfectly formed, never cease to make regular demands upon the blood; indeed their office is as constant as that of digestion, till the decay of the natural powers of life, although the purpose for which they were intended be not consummated. If there be any difference between them and other organs, it is that they are subject to occasional and periodical changes, which tend more than any thing I have yet mentioned to give an internal distribution to the blood.

C. The natural excitation of an internal viscus produces the same local alterations that the morbidly-excited action of any part of the system occasions in the condition of its organization; we have in both increased action and a highly vascular appearance; the striking dissimilarity between them is in the consequences which succeed. The disorders of the one subside with the cause which gave them rise, but those of the other are followed by febrile symptoms, a derangement of other viscera, and sometimes with disease of its own structure. From this general view of the changes which are peculiar to the animal economy at a certain period of life, it must be obvious to the reflecting mind, that these 
changes will lessen the distribution of blood upon the surface of the body, because the internal necessities are great, and therefore we shall have, from this time till the destruction of [organic existence, a state of circulation which may be designated, in contra-distinction to the former, internal.

CI. At this period of life, approaching to its acme, the respiration has become slow, the contractions of the heart less frequent by almost one half, the animal temperature on the whole less, and the appearance of the body is at variance with those indications which the infantile frame exhibited. We have now the thoughtful, pale, or sallow countenance : if not constitutionally incorporated, it occurs too frequently not to be remarked, and it is accompanied by too many internal derangements not to be felt, or traced to its appropriate source. But we have not only derangements, but diseases that demonstrate more clearly the correctness of the division of circulation at these different states of life, into external and internal. But, before we treat of these, we may advert to the exercise of the mental faculties, and the influence which they possess in controlling the sanguiferous system.

CII. The sports, pursuits, or feelings of the child, may be regarded as a general class of excitants : they are either attended by muscular exertion, which is favourable to the oxygenation of 
the blood and its general diffusion, or they are characterized by the buoyant or highly stimulating sensations of the mind, which accelerate the function of respiration, or at least tend to maintain it in an equable and vigorous condition. The child is subject to griefs, but the expression of these is not indicated by thoughtful taciturnity, by retirement from ordinary amusements, or by those deep inspirations which deteriorate the properties of the vital fluid at a later period of life, but are indicated by those very acts which tend, even more than its usual sports and feelings, to diffuse new life and energy throughout its system, and to maintain that external circulation of which we have spoken.

CIII. The influence of the mind in the child is almost invariably beneficial to its constitutional necessities, while the agency or exercise of this principle is too often injurious in its effects at a later period. The cultivation of intellect, independently of the mortifications and disappointments almost inseparable from it, is pursued at the expense of many moments that would be better devoted to sleep or amusement; but even if we suppose the mind to be passive from the indolence of its possessor, it is still necessarily exposed to a variety of sensations that are disagreeable or painful, and the universal tendency of these is to bring the blood from the surface of 
the body, to surcharge the internal organs, and thus to impede the proper changes of the blood and its dependent functions. Whenever the mind acts to this extent, a morbid congestion or disease of internal parts occurs; but if the cause which operates be much milder in its consequences, it is still liable to influence the natural internal determination or circulation of blood; I say natural, because I have already shown, that, as we recede from infancy to manhood, the digestive and excrelory functions augment, and others which are of importance to the animal economy are developed and brought prominently forward, the individual or collective support of which is derived from the blood; and therefore this change must modify the previous distribution, by creating new internal wants, and presenting new objects as forces of attraction or powers of control.

CIV. If the above view be correct, like all principles which are true it must be capable of application ; and if it be proved that the various diseases to which the different ages are exposed have no relation with the peculiarity of the circulation, it will then show that my observations and opinion are inaccurate ; but if it be acknowledged that the nature or character of the disease depends upon, or is influenced by this state, an opposite conclusion must be drawn.

C.V. From fourteen to eighteen years of age youth is liable to be affected with epistaxis 
and at the latter end of this period haemoptysis is not at all uncommon. The former is the consequence of a still remaining vascularity of those parts of the body which are most external, and is occasioned by the first disturbance which takes place in the system from a change in the mode of circulation, as indicated by a fulness of the head, and its dependent symptoms, such as dizziness, imperfect vision, troublesome or frightful dreams; and is also frequently accompanied with irritation of the trachea and bronchia, as proved by an occasional cough.

CVI. Epistaxis is generally produced by exercise, coughing, speaking, violent passion, and other similar causes. The system being excited by these, a quantity of blood greater than what is natural is determined to the surface of the body, and moreover must be evacuated for the safety or well-being of the animal economy; and as there is no other mucous membrane of the body equally vascular, and bearing the same relation to this extended surface, the blood flows from the delicate net-like membrane of the nostrils.

CVII. It is not my intention to enforce the correctness of this opinion by enumerating the different kinds of practice found most beneficial in its cure. This will occupy our consideration in a subsequent treatise; but I may observe, that the means employed, such as bleeding, application of cold and internal refrigerants, are precisely 
such as are calculated to destroy the condition of the circulation on which the hemorrhage depends.

CVIII. From sixteen to twenty-five, haemoptysis is the most frequent ; and it is at this period that the regular internal circulation begins to establish itself; and when the frame is so constituted as not to admit, without disturbance, this alteration, or when an individual is highly plethoric, this phenomenon occurs. It may probably be asked, why does not the system relieve itself as it did in the former instance? the answer to this is simple,-the natural vascularity of the surface of the body no longer exists : it is transferred more particularly to the internal organs; and whenever this normal modification is excited by any of the causes which promoted the former disease, the demand is made upon the lungs, as being the most vascular, the most delicate, and, from their situation, bearing the nearest relation to those parts which are oppressed, or rather to that state of the circulation which is to predominate till the decline of life.

CIX. The sensible and delicately formed frame of the female is protected, to a very great extent, from such disorders. Nature has been extremely provident in preserving this part of the creation from derangements to which her constitution would be unequal. When we are suffering from changes in the mode of circulation, which the 
strength of our constitution quickly overcomes, the various organs peculiar to her sex are developed; and from the organization, and extreme vascularity of these at certain periods, the oppression of the internal circulation is relieved by an excited action of these organs, often long before their operation is required for the ultimate ends of Nature. We thus perceive, that the vigour of the one and the delicacy of the other are secured by different designs: the former by the natural robustness of the frame, and by the sports and pursuits proper to that sex, which tend particularly to maintain a moderately external and uniform condition of the circulation; and the latter, by the influence of functions that might be called premature in their formation, if we considered only the other purposes which these are intended to fulfil.

CX. The acute and chronic diseases of both sexes from sixteen to twenty-five, and the various slight derangements to which the system is exposed, may be alluded to as presenting addi- . tional proofs in corroboration of this view. Inflammation of the lungs, or thoracic viscera, is not at all unusual; but this may be determined at every age of life, and therefore may be said to arise rather from the agency of occasional causes than from any constitutional predisposition. But it is between the above-mentioned periods that consumption is the most common 
and fatal; and unless there were some general character of the system favourable to the production of the disease, we cannot conceive it possible that one-fourth of the population of Europe should annually die of phthisis, as is stated by $\mathrm{Dr}$ Young. The exciting causes in civilized life are numerous; but unless there were a predisposition on the part of the system to the development of such a malady, these causes would not act so extensively as to occasion the most general and destructive chronic disease to which humanity is subject. The general and local means that have in all ages been found the most beneficial in retarding its progress, or the most powerful in effecting a cure, have produced their effects because they have universally been such as tended to equalize the circulation of the blood. Gentle exercise of the body, removal to a more temperate climate, sudorifics, emetics, the warm bath, and warm-aired rooms, friction, and the application of stimuli to the chest, have been recom- mended, and are allowed to be the most effectual in controlling or removing the affection. The action of these is to overcome the morbid internal distribution of the blood, and, by diffusing it more generally throughout the system, enable the lungs to regain the vigorous exercise of their function.

CXI. The greater number of the disorders of the female are to be attributed to irregularities 
in the uterine functions, all of which have the tendency to increase the natural internal circulation or determination of blood, giving rise to disorganizations of the lungs, weakness of digestion, deranged secretions of the abdominal viscera, palpitation of the heart, and disturbance of the mental powers. It is not necessary to insist further on the correctness of this opinion, by adducing other illustrations: the minute and efficient investigation will be found, not in any single chapter or view, but in examining the general principles which are proposed, in the variety of aspects which different diseases, seasons, and circumstances present.

CXIII.If we consider the nature of the diseases which occur after the prime of life, which arise simply from the extension of the same principles that we have traced from youth to maturity, we shall be agreeably surprised to find, that Dr CuLLEN referred the greater number of these diseases to a venous plethora, depending, as he supposed, on a difference in the proportional density of the coats of the arteries and veins. He imagined that in early life the coats of the veins have a proportional density greater than those of the arteries, on which account the plethoric state of the latter is to be regarded as the cause of many constitutional derangements and diseases; but at a subsequent period the density of the coats of the arteries becomes superior to that of the veins, and 
we have then a class of diseases called passive Hœmorrhagies.

CXIV.The effects of this venous plethora of Dr Cullen are precisely the same that we should anticipate to happen from a knowledge of the general changes in the circulation, as stated in this chapter; and it is for the reflecting reader to judge, whether the present coincidence ought to be regarded as confirming the views which I have attempted to establish.

CXV. In old age the motions of the heart are less forcible; the capillary system of the lungs less vascular ; " and the ability or disposition to exertion is diminished. It is therefore manifest that the blood is propelled with less energy by the influence of its moving powers throughout the system. The properties of the vital fluid are also less stimulating; and these conditions, combined with the comparative inactivity of the body, are well calculated to augment the natural internal determination of the sanguineous fluid. But we are not to suppose that the system in the decline of life contains the same quantity of blood which it did in its prime; the more confined operation of those organs necessary to digest and

* En examinant les cellules du poumon, j’ai été frappé de la variation de grandeur qu'elles éprouvent par les progres de l'âge; leur nombre est en raison inverse des années. Il resulte de ce fait que notre poumon doit acquerir, à measure que nous vieillissons, une légèreté specifique trés grande.-Journal de Physiologie Experimentale. Par F. Magendie, 4th Number. October, 1821. p. 80. 
assimilate has gradually been diminishing the proportion, to allow the lungs and heart to act with moderate correctness.

CXVI. From the preceding views we may draw the following conclusions:

1. That the blood in all young animals is generally diffused through the system, on account of the internal necessities making little demand upon this fluid; and that the character of this distribution is changed in proportion to the development of these necessities.

2. That, at the maturity of the animal frame, the internal organs are more vigorous than at any other period of life: and that, as the natural or diseased action of these is augmented, if unaccompanied by fever or exercise, the blood in all cases is determined to them in greater quantity than natural, either maintaining the regular internal circulation, or extending this to a state of aberration.

3. That, at the decline of the powers of life, the blood is more internal in its circulation than at any other period, from the concurrent influence of the previous gradual changes tending to promote this effect, and from the imperfection of those functions essential to renew its qualities and facilitate its motion.

CXVII. From these deductions others are to be drawn, the consideration of which will occupy much of our attention. Those which we shall at present consider are the following:

1. That, if the blood be more equally diffused in infancy throughout the system, it is obvious that a much 
greater proportional quantity will be distributed upon the surface of the body at this period of life, and consequently a greater quantity will be exposed to the action of heat or cold.

2. That exercise. and stimuli of every description will produce a factitious condition of the system, in character somewhat similar to that which is natural to the child. 3. That the general application of heat and cold will be injurious or beneficial according to the distribution of the blood upon the surface of the body and the degree of energy possessed by the internal organs.

CXVIII. Having premised these general principles, we shall be enabled to understand the influence of seasons in modifying the distribution of the blood.

The temperature of summer enlivens the animal and vegetable world, because the organic life of both is regulated by the same physical laws, and heat is as much a stimulus to the generation of motion in the one as in the other,-and from motion and warmth spring every changethat is necessary to preserve existence or maintain its attributes.

CXIX. The hibernating animals, and such as retain during the severity of winter the general powers of life, are enabled, by the greater warmth of spring, to resume their different functions. The former are again animated, and the latter, in addition to their present faculties, display, like the plant, an exuberance of life. 
CXX. The influence of warmth in animals operates upon the surface of the body and the organs which are fitted to improve the qualities of the blood. By exciting the surface, the action of the capillaries is universally stimulated; and the consequence of this is, that the internal organs participate in the change, as they are necessarily relieved of a portion of blood, from its more equal diffusion, and are rendered more capable of oxygenating, transmitting, and assimilating that which they receive. By acting upon the lungs, a direct stimulus is applied to their capillary vessels, which are intimately concerned in effecting the various changes of the blood, and directing the mode of its distribution ; and in so far as these are accomplished, the effects arising from the external cause will be more or less permanently or generally established. If these alterations have taken place, it must be allowed that the nervous system will soon evince a corresponding condition, as the result of these alterations; and we observe; that whatever depends on motion or sensation, becomes unusually energetic.

CXXI. Suppose a plant and an animal to have been brought prematurely forward, and afterwards to be exposed to a degree of cold rather severe for the season, they are both influenced in the same way by the same exciting and proximate causes. The surface of both is highly vascular; and as a greater proportional quantity of 
fluid is subjected to the action of cold, a greater quantity will be successively acted upon until the minute vessels are constricted by this agent; and when this happens, the plant droops, and the bird forgets its song. Every phenomenon of organic and animal life is regulated by, or depends upon, the nature and motion of the circulating fluid peculiar to the individual being.

CXXII. From this view it appears that summer has the tendency to diffuse the blood in greater quantity over the surface of the body; and this being the case, an individual will at this time be more liable to internal disorders and inflammations than in the middle of winter, from the occasional application of the opposite temperature. It is an ascertained and acknowledged fact, that, in autumn, inflammations of the thoracic viscera, fevers, and dysenteric affections, are more common than at any other season.

CXXIII. Previous to autumn, the system has experienced a general stimulus : accelerated circulation and augmented heat are its consequences; and as these conditions have existed for a long time, they have, in some measure, become established, and, therefore, cannot be expected to return immediately to another state which is to adapt the animal economy to different circumstances. It sometimes happens that autumn does not approach in a benignant or gentle manner, but, on the contrary, is accompanied by cold and damp 
nights, unusually severe; and, whenever this occurs the circulation is taken by surprise, * the blood is suddenly determined to the internal organs, and that which is the most susceptible to the impression becomes the seat of disease. In this way arise the frequent coughs, inflammations of the lungs or bowels, dysenteric symptoms, and fevers incident to that season.

CXXIV. When the constitution has been some time accustomed to the wet and cold days of winter, it is capable of resisting the influence of these with great security. If the system had not undergone a change in the mode of its circulation, it is impossible to conceive by what means it could withstand the operation of those causes to which it is exposed, since we observe so much internal disease occasioned by similar, but much weaker agents, in the more temperate seasons.

CXXV. Dr Currie, in treating of the subject of cold affusion in fevers, is correct in the observations which he makes regarding the conditions of the system which ought at all times to regulate its employment; but he does not appear fully acquainted with the principles on which the truth of his observations rests. He remarks, that it may be resorted to with safety and benefit when the temperature of the body is above the

* Nam fere (autumno) meridianis temporibus calor ; nocturnis atque matutinis, simulque etiam vespertinis, frigus est, corpus ergo, et aestate, et subinde meridianis caloribus relaxa: tum, subito frigore excipitur."-Celsus, Lib. II. Cap. I. 
standard, and when the circulation is vigorous, if these symptoms have not been preceded by profuse perspiration or great fatigue.

CXXVI. The augmented temperature and circulation are proofs that the thoracic organs are unusually or morbidly active; and the great quantity of blood which is distributed upon the surface of the body is sustained there by its highly stimulating properties and the force with which it is propelled. When an individual, with a constitution thus circumstanced, submits to the application of cold, it produces a good effect, as it diminishes the heat of the system and the strength of arterial action, without possessing sufficient influence to oppress the energies of the vital powers. But when the body has been much fatigued, or if it has perspired freely, the application of the same means is injurious. After fatigue and perspiration the blood is transmitted with less vigour and less stimulating properties to the surface, on account of the diminished action of the lungs and heart; and, therefore, if cold be recommended in this state of the system, it will disappoint the expectations of the physician, because it will determine the blood internally, and control still more the weakened function of the lungs and heart, so that the oxygenation and the propulsion of the blood will be much impeded. 


\section{CHAP. V.}

\section{Temperature at Different Ages.}

CXXVII. The present subject is one of great interest and importance. The principles by which we shall be guided in the investigation are connected with those which have been already stated, and will strengthen materially the reasoning previously employed.

CXXVIII. The temperature of the body at different ages has occupied very much the attention of Dr Edwards of Paris. Indeed, the experiments which he performed to elucidate this subject, and the consideration and application of the laws which he deduced from them, compose at least one-third of his work, - which is undoubtedly the best in any language for the multiplicity, variety, and accuracy of experiments ; but these, unless they lead to general laws, or unless they are brought forward to support such, are of little consequence, except in so far as they enable others to think.

CXXIX. In the chapter on secretion, I have shown how Wilsov Philip was mistaken in his opinions concerning the dependence of digestion on the eighth pair of nerves, from judging by effects, without taking into account, in any single instance, the general disturbance induced in the 
system by such an operation; and I shall now endeavour to show, that one great error which pervades the work of Dr EDWARDs, concerning animal temperature, is referrible to a similar cause. This gentleman states, that the faculty of producing heat is invariably less in young animals than in adults; and the chief proof which he gives is, that if young and old animals be exposed to the same degree of cold, the temperature of the former almost immediately falls, and continues to do so unless they be removed to a warmer medium, while that of the latter is little if at all affected by the refrigerating influence. This constant result made him conclude, that young animals do not generate an equal quantity of heat, because he supposes, if this were the case, they would both bear with the same facility the exposure to cold. From the development of other views, it will be,clear, that they may generate the same proportionate quantity of heat, and yet may be unable to bear the same extent of cold.

CXXX. Dr EDwards took the temperature of 20 adults, and found the medium to be $36^{\circ} .12$ centigrade thermometer; and from 10 infants whose temperature was taken in the same way, the medium was found to be $34^{\circ} .7$. In these experiments the thermometer was placed in the armpit. There are many objections to this mode of ascertaining the degree of animal heat. The part is particularly subject to perspiration, which may modify very much the results; or if the arm has 
been removed from the contact of the body it will be cooler than usual, or if it has been long applied to this it will be warmer at one time than another. These circumstances are of sufficient importance to occasion great variations in the indications of the thermometer, and consequent fallacies in the reasoning. The plan which I followed appears to me more correct, but it is certainly very tedious. Mr Morr, surgeon-accoucheur to the Lying-in-hospital, Edinburgh, had the kindness to allow me the opportunity of taking the temperature of infants. The temperature of the body was at all times estimated by the indications which the thermometer gave in the mouth when the infant was asleep. To make the instrument as delicate as possible, it was dipped for a moment before it was employed into a cup of warm water, from 5 to 10 degrees above the animal heat. The bulb being thus slightly warmed, did not awake the infant by its application, and was made much more sensible than the most delicate thermometer could otherwise have been. The same method was, in the greater number of instances, attended to in taking the temperature of adults. The child would sometimes be disturbed, but very rarely, by the introduction of the thermometer. Whenever this was the case, the attempt was postponed till the next visit. I have also noted the age of the infant, the number of its respirations, and the state of the constitution. 
Days Old. Temp. of the Body. Respiration per Min. Remarks.

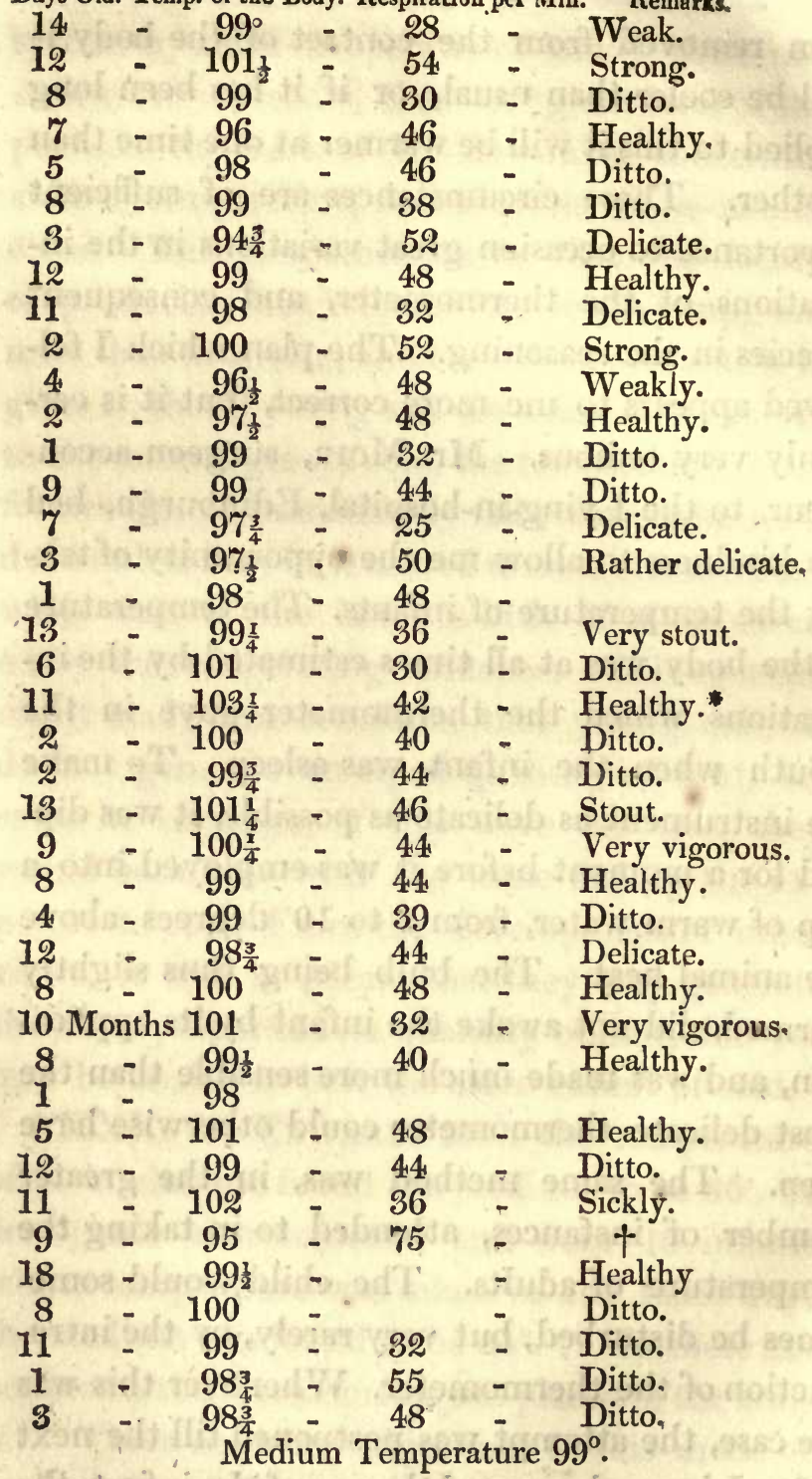

* The mother of this child sat close to the fire, but I cannot say whether this was the reason that the temperature was so very high. I tried it twice to be sure of the accuracy of the results.

+ This child seemed remarkably stout, but it was evidently unwell. Its respiration was short and imperfect. 
The following Table gives the Temperature of the same number of Adults.

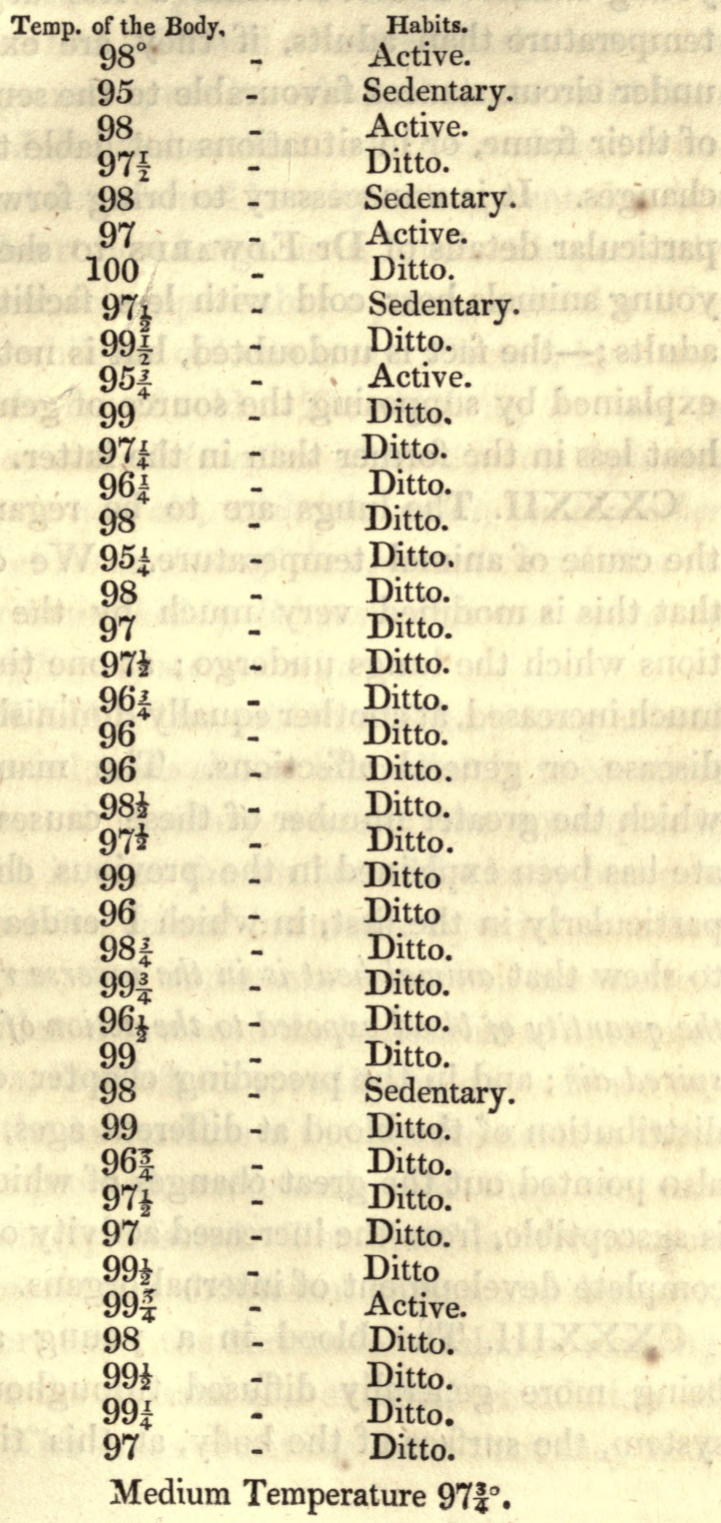


CXXXI. From these tables it is evident that young animals do not indicate a less degree of temperature than adults, if they are examined under circumstances favourable to the sensibility of their frame, or in situations not liable to great changes. It is unnecessary to bring forward the particular details of Dr EDwards to shew that young animals bear cold with less facility than adults;-the fact is undoubted, but is not to be explained by supposing the source of generating heat less in the former than in the latter.

CXXXII. The lungs are to be regarded as the cause of animal temperature. We observe that this is modified very much by the alterations which the lungs undergo; at one time it is much increased, at another equally diminished, by disease or general affections. The manner in which the greater number of these causes operate has been explained in the previous chapters, particularly in the first, in which $I$ endeavoured to shew that animal heat is in the inverse ratio to the quantity of blood exposed to the action of the inspired air; and in the preceding chapter on the distribution of the blood at different ages, I have also pointed out the great changes of which this is susceptible, from the increased activity or more complete development of internal organs.

CXXXIII. The blood in a young animal being more generally diffused throughout the system, the surface of the body, at this time of 
life, possesses a greater proportional quantity than at a subsequent period. If this be allowed, it is manifest that cold will simultaneously act on a much greater quantity of blood in the child than in the adult ; and as this mode of circulation is natural, not determined here by an augmented action of the heart and lungs as in fever, it is perfectly consistent to suppose that a young animal will be cooled, while an old one is not affected, by a moderate degree of cold. The child may be said to expose an extended surface of blood to the influence of the external air, while the adult, on the other hand, has the surface of the external circulation relatively diminished by the more vigorous operation of the different internal organs.

CXXXIV. The cold to which a young animal is subjected tends almost immediately to constrict the external capillary vessels; the consequence of which is, that the surface of the body becomes pale, and the animal intimates, by cries or other symptoms, that unpleasant sensations are excited; and, unless we remove the individual to a warmer medium, fatal effects speedily follow. If we examine the indications of the thermometer as these changes proceed, we remark that they are correspondingly affected. The blood is driven upon the thoracic and abdominal organs, and destroys, in a short time, the harmonious balance existing between the internal and external circulation of blood. The lungs receiving more than they usu- 
ally possess, the additional quantity necessarily impedes the oxygenation and circulation of the blood; and, in proportion as these essential conditions are disturbed, we shall find that the faculty of generating animal heat is diminished, because this is more powerfully infuenced by the more general distribution of the blood in early life, and not that it was originally less, as contended for by $\mathrm{Dr}$ EDwarDs.

CXXXV. To prove the correctness of these principles, we shall examine the various causes which, agreeably to Dr EDwards' opinion, modify the generation of animal heat. It is stated by him, that the temperature of animals is capable of being most diminished by the artificial application of cold in summer, because the power of producing heat is less at this season.

CXXXVI. Summer produces a character of circulation essentially similar to that peculiar to infancy and childhood. The capillary vessels are not constricted, as in winter, thereby determining the blood within, but are excited to greater action from the application of heat; therefore the blood is more equally diffused throughout the system, offering, as in infancy, an increased surface of blood to the action of cold so applied.

CXXXVII. An animal, in accordance with this explanation, will suffer sooner from the influence of artificial cold in summer than in winter; and this is fully verified by the experiments 
of Dr EDwARDs, although he assigns lessened production of heat as the cause. The principle I propose immediately becomes of practical utility, whereas those views which have hitherto been given as explanatory of the same phenomena have indeed little reference to the different states of the system in health or disease. We now understand that young animals have an equal, if not superior faculty of producing heat to adults, and yet, at the same time, have not the power of bearing the same severity of cold. The true reason of this being known, we shall be enabled to regulate the application of heat and cold to the body, from a knowledge of the principal conditions of the system on which they have to act ; and we shall also be enabled to judge accurately of the beneficial or injurious influence of these, from a knowledge of the dependence of the digestive and assimilating functions on the temperature of the blood and the mode of its distribution.

CXXXVIII. During the winter of 1828-29, the cold in some nights was extremely severe. The thermometer, in the room in which I kept the rabbits for experiments, was often as low as $37^{\circ}$; in an apartment adjoining to this water was frozen to a considerable thickness. During the continuance of this weather I lost a great number of young rabbits, and the old occasionally died; and all, except the most vigorous, were far 
from having a healthy appearance. I frequently took the temperature of rabbits under a variety of circumstances, and I generally found that the young and the delicate evinced a low degree of heat during the prevalence of cold, while the old exhibited few changes, exposed to the same agent.

The difference in the results were attributed to alterations of the circulation; and believing these to be the cause, I endeavoured, by the following experiments, to show that a medium temperature, by allowing the blood to diffuse itself more generally upon the surface of the body, would certainly increase the faculty of generating heat, as it relieves the internal organs on which this depends.

The temperature of twelve rabbits was taken that were equally exposed to cold, $43^{\circ}$; and the same three days after were kept in a room at $67^{\circ}$ for two hours.

\begin{tabular}{|c|c|c|c|c|}
\hline $\begin{array}{l}\text { Temp. of } \\
\text { Room, } 43^{\circ} \text {. }\end{array}$ & Colour. & Appearance. & $\begin{array}{l}\text { Temp: of } \\
\text { Rabbits. }\end{array}$ & $\begin{array}{l}\text { The same } \\
\text { exposed to } 67^{\circ} \text {. }\end{array}$ \\
\hline 2 Months old, & Black, & Healthy. & $99^{\circ}$ & $102^{\circ}$ \\
\hline Ditto, & Black \& white, & Ditto, & 99 & 103 \\
\hline Ditto, & Black, & Very vigorous, & 102 & 103 \\
\hline Ditto, & Ditto, & Small, & 98 & 103 \\
\hline Ditto, & Ditto, & Healthy, & 98 & 102 \\
\hline 3 Months old, & Grey, & Vigorous, & $102 !$ & $103 \frac{1}{2}$ \\
\hline Old rabbits, & Black, & Ditto, & 104 & 104 \\
\hline Ditto, & Grey and white, & Ditto, & 103 & $104 \frac{1}{2}$ \\
\hline Ditto, & Grey, & Ditto, & 108 & $104 \frac{1}{2}$ \\
\hline Ditto, & Black, & Very vigorous, & 103 & 105 \\
\hline Ditto, & White \& yellow, & Vigorous, & 103 & 104 \\
\hline Ditto, & Yellow, & Healthy, & 100 & 103 \\
\hline
\end{tabular}


In the following experiments, instead of bringing the rabbits from the room in which they were kept, I confined them in a box, in the same situation, for two hours; and the results were as follow :

Temperature of air in room $43^{\circ}$.

Temp. Rabbits Temp. Rabbits Colour. Appearance. in Room. in Box.

\begin{tabular}{|c|c|c|c|c|}
\hline Months old, & Black \& white, & Healthy, & $101^{\circ}$ & $104^{\circ}$ \\
\hline Ditto, & Grey \& white, & Ditto, & 101 & $102 \frac{1}{2}$ \\
\hline Ditto, & Black \& white, & Ditto, & 102 & 102 \\
\hline Ditto, & Grey \& white, & Ditto. & 101 & 103 \\
\hline Ditto, & Ditto, & Strong, & 103 & 104 \\
\hline Old Rabl & Grey \& & Healt & 102 & 108 \\
\hline Ditto, & Ditto, & Ditto, & $103 \frac{1}{2}$ & $103 \frac{1}{2}$ \\
\hline Ditto, & Ditto, & Ditto, & 103 & 105 \\
\hline Ditto, & Grey, & Fine buck, & 103 & 104 \\
\hline Ditto, & White. & White, & $103 \frac{1}{2}$ & 102 \\
\hline
\end{tabular}

The temperature of the box, in both instances, was $67^{\circ}$. In the first experiment, the rabbits that were two months old indicated a rise of four and five degrees in the short space of two hours; but in the second, in which rabbits of three months old were employed, we observe that the influence of the increased temperature produced less decided effects, because they possess, at this age, a constitution approaching to that of adults.

CXXXIX. It is stated by Dr EDwards, that young rabbits of twelve or fifteen days old, "se soutienne à peu-près égale à celle des adultes, au-dessus d'une température extérieure moy- 
enne." It is evident, from the preceding tables, that rabbits of two and three months old do not bear equally well the severities of winter. I have not only ascertained this fact by direct experiment, but I have observed its correctness, from remarking a variety of phenomena connected with these animals. If it be allowed to Dr EDwards, that young animals, from birth to the age of fifteen or thirty days, do not possess the faculty of generating animal heat to a degree equal to adults, how will he consistently explain the difference which we observe between rabbits of three months old and others full grown, on being exposed to cold? If, at three months, the faculty of generating heat is as perfect as at nine, what is the nature of those circumstances which modify the capability of the animal for bearing diminished temperature? These are questions of the greatest importance; but they are not answered by Dr EDWARDS' views. The explanation is found in the present principles. As we ascend from birth to the full development of the physical powers of the system, the circulation is less generally diffused, or, in other words, the greater proportional quantity is given to the internal organs for their support and development. It is, therefore, obvious that an animal will bear cold with a facility, cateris paribus, proportionate to the extent of this change.

CXL. Having shown that the phenomena which animals present in summer, in regard to

* Dr Edwards, p. 136. 
cooling, are easily explained by the present principle, we shall now pass to several others brought forward by Dr EDwaRDs, not in support of his own opinion, but as facts. He states that exterior heat supplies the deficiency in the faculty of producing.*

When we apply warmth to the surface of the body, without in the least modifying the temperature of the air we inspire, we augment the generation of animal heat. This effect is not easily perceived when the system is naturally excited, because the difference we can produce in the mode of circulation is too slight to influence, in an appreciable manner, the generation of this principle: but if this be employed when the powers of life are much oppressed by a great determination of blood to the internal cavities, the beneficial influence of this agent is strikingly displayed. Physiologists and physicians have long known that such means diminish internal congestion; but they must have supposed that this was confined chiefly to the abdominal viscera, as they appear unacquainted with the general principles and their application, fully treated of in various parts of this work. Friction, applied to the body, produces similar consequences to those of heat; and this shows that the action of heat affects the internal production principally by bringing to the surface an additional quantity

* Dr Edwards, p. 480. op. citat. 
of blood, and thereby lessening the proportion exposed to the air in the lungs, according to the views developed in the first chapter.

CXLI. The diminution of the temperature of animals is less injurious in very early than in advanced life, because it is found that at the former period they sooner regain their accustomed standard of heat. In speaking of this, Dr EDwa RDs observes, "Nous voyons que c'est à mesure que la faculté de développer la chaleur s'accroît que la faculté de supporter l'abaissement de la temperature du corps diminue."* After these observations, he alludes to the necessity of such a law with respect to those animals which are forced to leave their young in search of food. The necessity of such a provision in the constitution of the young is evident; but the simple statement of this is no approach towards an explanation of the principles on which it depends. The faculty of bearing a diminution of temperature is not regulated by the condition he states, vix. the power of generating heat. I have already shown, in the previous tables, that infants, in general, have a superior temperature to adults; and that rabbits vary little, if at all, at different ages, if exposed to a medium temperature. The truth of this I have proved by direct experiment ; and therefore it is inadmissible to suppose that young animals suffer less than adults from the action of cold because they ge- 
nerate less heat, and that the power of bearing cold diminishes because the production of this principle is increased. This must be allowed to be the meaning of Dr EDwards, if we agree with him that young animals have an inferior power of evolving caloric.

CXLII. When a young animal is subjected to cold, it is quickly affected; the blood is speedily determined to the internal organs, and the body shows a great diminution of temperature : but the very conditions which promote this sudden change are those which are favourable to the re-establishment of the normal functions. The surface of the body at this period is naturally vascular, and on this account is more sensible to the application of Theat; and it is this character of the circulation which explains the phenomena connecled with the present subject.

CXLIII. When the temperature of an adult is much reduced, the system recovers with difficulty the possession of its ordinary powers. The vigorous frame of a full grown animal is not readily controlled or influenced by cold : it consequently follows, that if it be oppressed by this agent, its degree must have been severe, or its employment long continued-circumstances which we are not compelled to take into consideration in reflecting on the changes which one of tender years presents. 
CXLIV. The system of the adult having been thus powerfully acted upon, and the surface of the body being less vascular at this period of life, it cannot be expected that external warmth will immediately solicit the return of the sanguineous fluid; the degree and continuance of its application must be proportionate to the conditions of the cause to produce a beneficial result.

CXLV. It is demonstrated by experiment, that when an animal has been cooled several times to a point beneath the regular temperature of the body, it becomes more difficult to re-establish the usual degree of heat, and this is what we should have anticipated from a knowledge of the very principles propounded. If we suppose a young animal to have been repeatedly, and at short intervals, exposed to a severe refrigerating influence, the blood is every time determined to the internal organs, and the means which we subsequently employ, to overcome the consequences induced, imperfectly restore the functions of the body; and if these means be suspended, and cold again be applied while the system has but partially regained its energies, a similar but more serious effect follows, as the heart and the lungs are successively oppressed by new difficulties before they had risen superior to those occasioned in the first place. The repeated application of cold places the constitution of a 
young animal precisely in the same situation as that of the adult when distressed by the same cause.

After this explanation, it is scarcely necessary to say, that, if an animal of mature years be subjected to the same conditions, it is more than probable that we shall ultimately fail in our endeavour to restore the action of the vital organs.

CXLVI. It is remarked by Dr EDwarns, that, when an individual has been exposed to a degree of cold disagreeable to the animal economy, the faculty of generating heat is diminished for some time, even after the system appears to have regained its accustomed temperature. In illustration of this opinion, he relates a case in which a young man is stated to have fallen into the Seine in endeavouring to walk upon the ice: from his vigour and agility, he speedily extricated himself from his perilous situation, and although his health was not affected, he was annoyed for three days with a continual sensation of cold. This physiologist gives no rationale of the phenomenon, except, "I La faculté de produire la chaleur n'a pas repris toute sa force; car on ne saurait, quelque temps après, s'exposer sans inconvénient à un degré de froid qu'on supportait auparavant sans malaise." * In this instance he states a fact which is evident to every

* Page 249. 
observer, and remarks that the focus of heat has its faculty lessened, without informing us of the precise nature and conditions of this power, whose office it is to distribute warmth throughout the system.

In the case which he brings forward, we have to consider the operation of two causes, the application of cold, and the impression of fear upon the mind; the former is an external depressing power, the latter an internal. The tendency of both is to disorder the functions of the heart and lungs, by deranging the equal distribution of the blood submitted to their influence. After the system appears to possess the usual degree of sensible heat, the ordinary state of the circulation is not fully developed; and it is therefore at this time, and from this cause, that the sensation of cold is felt, and that the power of resisting this agent is diminished.

CXLVII. In corroboration of these principles it is found, if an animal has its temperature reduced by the application of cold, that the diminution is successive without any additional severity being added to the uniform cause to account for the gradual increase of the effects.

If we suppose the agency of cold to be estimated by 20 , and the immediate reduction of the temperature by 30 , in considering the influence of the first we shall be enabled to give reasons for the increase of the latter. The im- 
mediate reduction cannot be denied to originate from the determination of blood to the thoracic and abdominal organs, and as the power which occasioned this alteration is still applied to the surface of the body, its depressing energy is still great, because it has to act on the capillary vessels, already enfeebled by their previous loss, but more enfeebled by the deteriorated action of the heart and lungs, whose office it is to supply them with blood calculated from its properties to stimulate the external circulation, and thereby to render it superior to the influence of the cold; but this state of the economy being absent, the refrigerating power continues to operate, and as this proceeds, unless reaction takes place, the effects progressively increase to 40,50 , or 60 .

CXLVIII. The explanation of this and the preceding phenomena does not require the consideration of that unknown and much magnified power, the vital principle. The path we have pursued has been simple and straight-forward, and the elucidation of the principles, if allowed to be correct, is immediately brought to bear on the purposes of life, while those views that have been hitherto entertained on this subject have little or no application to recommend them.

CXLIX. From the preceding observations it is evident, that the plan which Dr Edwards has employed to estimate the faculty which an animal possesses, at different ages, of generating 
heat, is inaccurate; and if this fact be considered established, it necessary follows, that the whole of his reasoning, connected with the present subject, is fallacious or imperfect. I hope it has been sufficiently proved, that the temperature of two individuals may be the same in degree, and the source to which this is attributed similar in both, and yet one may be almost frozen to death while the other is comparatively little affected; and, still further, that two individuals, as in the present instance, will recover, by the application of warmth, with different degrees of facility, without being compelled to suppose, with this physiologist, that the conditions which regulate this phenomenon are derived from the laws of the generation of animal heat.

CL. If the system of the infant and adult were precisely the same in regard to circulation, sensibility, and the chief of the organic functions, it would be consistent to employ the means which he has employed, and correct to draw the conclusions which he has drawn from them; but it is impossible to allow this mode of proceeding, since the systems on which he experiments are as opposite as the results which they give. The number of respirations, and the frequency of pulsations in a given time, the power and character of digestion, and the state of the internal organs, are too important to be overlooked in calculating the influence of an external agent. 
CLI. In advanced life the constitution is highly sensible to cold, but this susceptibility is not referrible to a state of the circulation analogous to that which predominates in the infant, but depends on other principles. At this period the lungs and the heart do not exhibit that energy which characterized their action at the commencement of manhood; the blood is transmitted sparingly, and with little force, to the surface of the body; and from this cause the capillary vessels possess little stimulus to resist the influence of cold, and, therefore, if this be applied, it is quickly accompanied with disagreeable sensations.

CLII. From the preceding observations we may draw the following conclusions :-

1. That young aninials possess an equal, if not superior, faculty to adults for the generation of animal heat.

2. That the facility with which an animal bears exposure to cold, is regulated by the distribution of the blood at different periods of life.

3. That individuals of the same class, and of the same age, differ in their power of resisting cold according to the character of the circulation and the force with which the blood is propelled by the heart.

4. That the ease with which animals are recovered after exposure to cold is modified by the previous condition of the circulation, and consequently is not regulated by their different powers of generating heat.

5. That the more sudden diminution of the temperature in summer, on the application of cold, is not explain- 
ed on the supposition that less heat is generated at this season than in winter, but is explained in taking into account the general and more equable distribution of the blood.

6. That the beneficial or injurious effect of cold will at all times be regulated by the principles here stated,

\%. That the power of generating animal heat is in no case to be estimated by the application of cold to the body, nor by the consumption of oxygen; a difference in the constitution, inappreciable to our senses, will render the former mode incorrect, and the magnitude and activity of the lungs are too intimately connected with other functions of the system, or with the variety of states influencing these, to allow the physiologist to deduce legitimate conclusions from such premises. The thermometer is the only means we possess to ascertain this point. 


\section{CHAP. VI.}

The manner in which the System is adapted to the influence of Cold.

CLIII. THE facility with which we support the varieties of seasons, or the extremes of different media, has, from the earliest records of observations, been continually adverted to, and has also been productive of many opinions. To ascertain the laws which regulate the capabilities of the system in withstanding the operation of cold, is even yet a subject of great interest and importance. Although modern science has thrown considerable light on the influence of certain agents, which more or less contribute to explain the difficulties, yet it must be acknowledged, that we have not hitherto possessed satisfactory principles which apply to general effects or ascend to general causes.

CLIV. In treating of the powers of the system in resisting the agency of cold, it is scarcely necessary to state, that the present chapter is a continuation of the principles previously brought forward in the two preceding, viz. "On the distribution of the blood at different ages," and "On the temperature of the body at different ages." Whatever facts may on the present occasion be 
adduced, can be considered only as further illustrations of the correctness of what we have already discussed.

CLV. The wisdom of Providence is shown in the structure of organic matter, and in the delicate adaptations appropriated to its support and development; but, in forming these, it has had regard to the general changes of the universe, which exhibit, in a striking manner, the wise designs of its pervading spirit. The seasons of the year follow each other in regular succession, for the well-being, not only of the vegetable, but also of the animal creation. If the order were reversed, the animal and vegetable constitution remaining as at present, neither the one nor the other could exist. The gradual steps, by which organized matter accommodates itself to the influence of external causes, would be disturbed, and a repetition of this disordered action would speedily annihilate the functions of organic and animal life.

CLVI. During the warm months of summer, the circulating fluids of animal and vegetable matter, as previously stated, are more generally distributed throughout its capillary vessels; in the former it has been considered as constituting a state of circulation designated external, in contra-distinction to that which is observed in the severity of winter. The powers of life would be as much endangered by a sudden transition from 
summer to winter, as from winter to summer, on account of the unprepared state of the circu. lation to adapt itself to such alterations. To obviate such evil consequences, spring and autumn are given. The mild and enlivening influence of spring may be said to give motion, and, at the same time, security to the different fluids to diffuse themselves, by which means animated $\mathrm{Na}$ ture is almost imperceptibly adapted to bear the more stimulating power of summer; and when this season has performed its revolution, the $e x$ ternal capillary circulation is modified by the in. fluence of autumn, and is thus prepared by the most gentle degrees to sustain the keen air of winter.

CLVII. If proofs were required to strengthen this opinion, it would not be difficult to adduce facts that strongly corroborate its validity. We observe, in a variety of instances, when the transitions of seasons are abrupt, a train of consequences that beautifully harmonizes with the principles propounded. It sometimes happens, while the external character of the circulation is maintained by the warm days of autumn, that the cold bleak winds of this season suddenly set in ; and when this occurs the circulation is taken by surprise, the blood is quickly determined to theinternal organs, from the enlarged surface which it offers to the influence of cold, giving rise to the various forms of catarrh, inflammation of the 
bronchia and lungs, and particularly to dysenteric symptoms. These diseases, at this period of the year, are so prevalent that they are occasionally supposed to be contagious, an idea by no means correct. That many individuals are affected with the same complaints at this period cannot be called in question, but the number is no proof whatever that these are propagated by contagion. The universality of the diseases is against the supposition, and contributes much to show their non-contagious nature. The causes which produce these affections are general, and the systems on which they have to act are in similar circumstances. If one individual be subject to dysentery or catarrh, from the condition of the circulation constituting a greater degree of susceptibility, it is reasonable to suppose that a thousand may be similarly situated: they possess the same liability, except in so far as this liability is modified by a difference of dress, mode of living or pursuits, or original constitution. The minute investigation of the subject does not strictly belong to this chapter, and therefore we must be satisfied at present with its general illustration.

CLVIII. If the unexpected cold of winter produces unfavourable results upon the system, we perceive that the unusually warm days of spring also occasion analogous effects. The sun, that gives premature development to vegeta- 
tion, tends also to promote an excitation of the animal frame, and both equally suffer from the same cause. The circulation of the sap in plants acquires greater motion, and more invigorating properties from the application of the sun's rays : these conditions form what is called its vitality, the energy of which is displayed in the evolution of the bud, leaf, and flower; but it is probable that these are put forth at a time when the continuance of the weather is little to be depended upon; and if to a few warm days succeed nights more severe than is compatible with their progress, we observe that they soon languish and die.

To say that they suffer from their extreme sensibility, is no explanation of the laws which regulate their economy. The capillary system of plants changes with the seasons, as well as that of animals. The minute vessels of its organization are charged with a peculiar fluid, and the qualities of this contribute to create others still less cognizable by microscopic means, presenting an enlarged surface of circulation to the influence of cold. The leaves of plants may be considered to compose their surface, and, from the number of these in spring, we may estimate the probabilities of danger.

The dread of the agriculturist is the too early appearance of vegetation. His fears are not excited by the mere symptom of germination, but к 2 
by the liberal expansion of leaves; and how often with anxiety does he look forward to the fall of snow to protect the tender plant. His observations, although unenlightened by a knowledge of those principles on which the effects depend, are valuable, as they are in accordance with views proposed to explain the nature of those principles.

CLIX. If we take a view of the animal economy at this period, in which vegetation displays its precocity, we shall observe that its more perfect systems are equally exposed to, and injured by the sudden change from heat to cold. The warmth of spring is a stimulus to the surface of the body and the functions of the lungs. The blood, that was previously concentrated to the more vigorous capillary vessels, becomes now diffused among the most minute, making the extremities and external parts of the body nearly as warm as the internal, which is never the case during the severity of winter.

The distribution of the blood having undergone this alteration preparatory to the fervid and uniform days of summer, is checked by the cold nights of spring ; from which cause, catarrhs, inflammations, and fevers are produced as in autumn. The only difference between these two seasons is, that autumn acts upon the system by repressing too violently the conditions of the circulation derived from summer, while spring, having changed too early the circulation charac- 
teristic of winter, disturbs it by re-establishing abruptly its former state.

In both instances the physical eonditions of the system are the same, an enlarged surface of blood is subjected to the agency of cold, without the capability of the one being proportioned to the circumstances of the other.

CLX. The knowledge of the opposite states of the sanguiferous system, at various seasons, explains, in a satisfactory manner, the powers of vitality in resisting the effects of cold. We are not necessitated to have recourse to the supposed existence of an ideal principle, and, by endowing this with convenient properties, to give the semblance of an explanation of the phenomena of organiclife. Nor are we compelled to grant to Dr Edwards that the greater or less consumption of oxygen, at the various seasons, is the cause why the frame conforms to external circumstances. This opinion, and many others connected with it, have been shewn to be incorrect in the preceding chapter. We perceive the vegetable and animal to possess corresponding general characters of circulation at different seasons of the year; and we also remark, that these characters are influenced by external causes in the same way.

CLXI. As we proceed from south to north, the constitution of man changes with the changing climates; his susceptibility of cold is dimi- 
nished by natural adaptations of the system, such as the more confined distribution of the blood, materially assisted by the manners, dress, and mode of living peculiar to the latitude of the country.

CLXII. In the most northern climates, the inhabitants do not enjoy the same extensive variety of seasons that others in more favourable situations possess. Spring and autumn are not the half of their year, - the former, to habituate, by imperceptible degrees and long duration, the constitution to bear the oppressive heat of summer,-the latter, by a similar wise design, to temper the approach of winter. The animal and vegetable life of these regions is enlivened but for a short time by the rays of summer, and exhibits little of the energies of the same invigorated by a genial clime, in the development of muscular power, or in the expansion of the intellectual faculties, or in the profusion of flowers and fruits. If it were possible for nature to be partial in her gifts, the miserable inhabitants of these climates might be adduced as an illustration; but if she has deprived them of the elevated charms of social life, the pleasures of refinement, or the luxuries of taste, she has given them fewer necessities, and these of a kind more readily supplied.

CLXIII. Nature has not only been provident in harmonizing the mind of man to his situation, but has also been careful to provide him with an 
abundance of whatever is required to protect the body from the inclemency of cold, and to support the vigour of the constitution from its influence. The food of northern inhabitants is substantial and nutritious; it is generally of an animal nature; the clothes in which they are dressed are the skins of different kinds of animals, or, if the labour of art be employed, they are such as are well adapted to defend the surface of the body. The internal organs being rather invigorated than stimulated by the mode of living, and the surface of the body having a moderate temperature, preserved by additional or warm clothing, the vital functions are enabled to continue their operations with slight modifications to the system.

CLXIV. The winter in Paris is frequently found less easy to bear with comfort than the same in countries farther north, where the thermometer indicates a lower degree of temperature. The explanation of this phenomenon depends npon three general causes: 1 st, The circulation is more exposed to the agency of cold, in this and in similar regions, from the greater heat of autumn, or its longer continuance: $2 d$, The clothing is light, and furnishes an insufficient protection against the severity of cold; and, lastly, the food which is taken is more stimulating than invigorating: all which circumstances are at variance with those which preserve the constitution in more tempe- 
rate climates from the evil consequences and painful sensations of cold.

CLXV. From the present view, it is not difficult to explain the more frequent occurrence of phthisis in countries that seem calculated to destroy the tendency to such an affection; but the circumstances which seem to give security to the system against such a disease are the very circumstances which promote it.

CLXVI. Much has been written on the action of cold; and it has been warmly contested, whether it is to be considered as a stimulant or as a sedative? It is manifest, from the development of the principles concerning the distribution of the blood at different ages and seasons, that cold, though primarily sedative, will produce a sedative or stimulant effect according to the character of this distribution and the energy of the thoracic organs.

CLXVII. The spasm of Dr CULLEN is the keystone of his doctrine of fevers. It was ably supported by himself, and attained great celebrity, and is even yet brought forward by his enthusiastic adherents, as possessing the same important rank in febrile diseases as first distinctly stated by him. How "a spasm of the extreme vessels proves an irritation to the heart and arteries," neither he nor his followers have explained in intelligible language. If we were to allow the 
existence of such a state of the vessels, we should be necessitated to grant that vegetables have also their spasms, for I have shown them to exhibit the same general laws of organization, and to be affected by the same general causes as the animal economy. But instead of entertaining for a moment such an idea, let the philosophical mind examine the evident operations of physical agents, and it will find that the assumption of this is equally as gratuitous as the vis medicatrix of the same author.

CLXVIII. The external application of cold, and certain states of internal disease, determine the blood upon the internal viscera, and thereby deprive the capillary vessels of their usual fulness; but the constriction which succeeds is not the cause of internal congestion or of fever, but is the simple effect. of a depressing power,- and in no stage of the disease is it to be regarded in the light of a cause.

CLXIX. We have, as yet, considered only the influence of a moderate degree of cold, and the principles by which the system conforms to these changes; but we must now examine the influence of an extreme degree, and endeavour to explain the concomitant phenomena. When an individual is exposed to intense cold, certain unpleasant sensations arise, such as pain or coldness of the extremities, and oppression of the chest; difficulty of breathing, insensibility of the feet, 
and a strong disposition to sleep, are a few of the prominent symptoms which are present.

CLXX. From the almost universal occurrence of the propensity to sleep, it has been imagined by most physiologists, but with little truth, that the nervous system is individually and directly acted upon by the refrigerating agent. It produces the same effects as a powerful narcotic; and the following explanation will show why these are not to be attributed to the nervous but sanguiferous system.

CLXXI. I have already stated, that the application of cold tends to determine the blood upon the internal organs; and if this be long continued, or severe, the quantity which is sent from the surface and extremities will be proportionate to the causes which operate; and, in all cases in which sleep is induced, the action of these causes has been incompatible with the proper performance of organic functions. The numbness of the extremities, the pale and collapsed state of the body, prove that the internal organs must have received more blood than natural; and, from the principles developed in the first chapter, it is obvious that every quantity above what is natural, tends to diminish the stimulating properties of the blood and the force of its circulation.

CLXXII. The difficulty of breathing, and the pain in the chest, support this opinion. From the first disagreeable operation of cold to the com- 
mencement of the tendency to sleep, the respiratory functions have been disturbed. The properties of the blood gradually become deteriorated; and, as these alterations proceed, the heart in the same ratio has its powers of propulsion diminished. From these conditions it necessarily follows, that the arterial blood which is sent to the brain, and other parts of the body, is deficient in its ordinary stimulating qualities. The action of the heart being enfeebled, it is less capable of transmitting the blood to the surface of the body, to counteract the injurious effects of cold. From this view, it is manifest that the lungs are, in the first place, affected by the disorder of the $e x$ ternal circulation; and this continues to increase according to the severity or duration of the cold, and is also augmented by the inability of the heart to relieve the congested lungs. The qualities of the blood having become less stimulating, they are less calculated to excite the action of the sensorial faculties; and, indeed, every part of the nervous systen has the necessary relations existing between it and the sanguiferous deranged, relations which regard the properties of this fluid and its quantity in partial circulation. Dr BEaupre, in his Historical and Mcdical Sketch of the Russian Campaign, gives an interesting description of the symptoms characteristic of the influence of cold. 
CLXXIII. Individuals exposed to cold are very frequently induced to take a liberal quantity of spirits, to protect them, as they imagine, from the depressing effects of this agent; but it very frequeutly occurs, that even a slight excess of these ultimately augments, rather than diminishes, its influence.

CLXXIV. No satisfactory explanation has yet been given of these phenomena : but the following, founded on the principles which have preceded, will probably make an approximation towards a rationale. Spirits, of what kind soever, excite the action of the heart, and whenever this takes place, the respirations must become either deeper or more frequent, in order to maintain the essential relations subsisting between the lungs and this organ. The results of these changes are, an agreeable internal feeling of warmth, and diminished sensibility to cold; the blood, that had before become rather internal than external in its circulation, is now, from the functions of the thoracic viscera being excited, propelled with energy to the surface of the body, and is more equally diffused throughout the capillary vessels, presenting a distribution similar to that which predominates in the child, and in all animal nature, during the heat of summer.

CLXXV. In the two preceding chapters it was fully stated, how the system of the child, 
and that of the adult in summer, are more susceptible to cold; and as I have now attempted to shew that the circulation induced by spirits is precisely of the same character, if the reasoning and illustrations in the former instance are allowed to be correct, it is impossible to deny that the conclusions to which we arrive in the present are also sound and legitimately drawn.

CLXXVI. Although the general phenomena in these instances are of the same kind, yet there are certain differences between the state of the system occasioned by spirits and that peculiar to childhood and summer, too important to be passed over.

The external circulation in summer, and in early life, arises from a permanent and general cause, or of certain organic conditions of the frame, while that which is produced by stimulants is from a partial and evanescent cause. As long as the blood is determined to the surface from the regular and increased action of the heart, the system does not materially suffer; but as this action, if the effect of spirits, very quickly subsides, the vascularity of the capillary vessels is not maintained by the uniform transmission of blood, and consequently the greater surface of blood exposed to the action of cold is left to contend against its influence, without possessing, as before, increased momentum, and highly stimulating properties. This view, conjointly with 
others that have been stated, appears sufficient to account for the diminished capability of the constitution to withstand an exposure to cold.

CLXXVII. There are certain states of the mind which tend powerfully to counteract the effects of cold. Agreeable sensations, or the strong exciting passions of the mind, act exclusively upon the nerves appropriated to respiration;-and, by improving the frequency of this function, or by changing the two actions of which it is composed, the blood is invariably better oxygenated, and distributed throughout the system with greater regularity and force. It therefore follows, that the debilitating effects of cold are counteracted as long as the action of the heart is increased by these means, and as long as it continues to circulate blood possessing such qualities.

In a violent paroxysm of insanity, the body is little affected by external agents. In attending the interesting lectures of M. Esquiror, at the hospital Saltpetriere, Paris, I had frequent opportunities of observing the powers which the body seems to acquire, during mental aberration, of resisting those agents which, in the natural state of the mind, never fail to be accompanied by painful feelings or evident effects. There are, indeed, several cases on record, in which individuals are stated to have escaped from confinement when affected with insanity, during the greatest severity of winter, without the protection of even 
their ordinary dress, and who were afterwards taken, and found to have been exposed for many hours to the bitter wind of the season, without suffering from the exposure. The explanation of this and similar phenomena will not be difficult, if we consider that, in a violent fit of insanity, the disposition of the patient promotes muscular exertion of almost every kind. Incessant speaking, constant pacing to and fro, and repeated attempts to break all restraints, are a few of those efficient causes that excite the action of the lungs and heart, and which are every way calculated to sustain the augmented stimulus of the blood and its general distribution. The insensibility of the mind to disagreeable impressions is probably another important cause, tending to protect the system from the effects of cold. Respiration is much disordered by two grand classes of sensations, the exciting and clepressing. The influence of the former is illustrated by the paroxysm itself; but when an individual, whose intellectual faculties are undisturbed, is subjected to a painful degree of cold, the various organic derangements awaken certain sensations in the mind of a depressing description, and these react upon the function of respiration, destroying its regularity by sighs and deep inspirations, and induce alterations in the properties of the blood and the mode of its circulation. But, in a fit of insanity, impressions of this kind are not communicated to 
the sensorium, or rendered acutely sensible to the individual. The absence of such a state of the mind allows the insane person to indulge his outraged feelings without these being controlled by the susceptibility of the body to the agency of external causes. 


\section{CHAP. VII.}

\section{On the Torpidity of Hibernating Animals.}

CLXXVIII. THE subject of torpidity has occupied, like that of the preceding chapter, the talents of the physiologist and naturalist, and, like the same, is enveloped in much mystery. The greatness of an effect too often blinds the mind in attempting to ascertain its cause, by mingling in the enquiry a degree of wonder or admiration; and I am disposed to think that the subject of torpidity has been investigated by some with a feeling of this kind. The regularity with which animals have retired to their convenient resorts, the duration of their repose, and the comparative vigour with which they have returned to active life, are certainly occurrences that cannot be regarded, by the reflecting mind, without a degree of wonder and admiration.

It is not my intention to give a complete history of the different opinions which have been expressed on this subject, nor to treat it so fully as the reader will find it discussed in Dr FLEMING's excellent work on Zoology. * I am anxious to examine a few of its most interesting and

* Vol. ii. page 45. 
important phenomena,-and for this purpose shall consider the three following subjects:

1. The nature and influence of those changes which predispose to, or precede torpidity.

2. The state of the system during the repose.

3. The nature and influence of those changes which predispose to, or precede the return to active life.

CLXXIX. The period at which hibernating animals undergo this extraordinary change is various in the different species, and even in those of the same species. The greater number exhibit a decline of the active powers of life, and retire to their winter retreats in September and October. At the latter end of autumn the temperature of the atmosphere is much diminished, and at this season of the year cold and bleak winds frequently occur, which tend still more to affect the animal economy.

CLXXX. I have endeavoured to shew that the circulation of blood at different ages possesses different characters, and, still further, that the generation of animal heat in man is regulated by its various conditions, and not by any power of the lungs independent of these conditions, or any power of the nervous system, as universally supposed. Since the correctness of this opinion cannot be doubted, we have facts on the one hand, and probability on the other, from analogy, to enforce the belief, 
that those animals, among the warm-blooded, whose temperature is low, or whose susceptibility to cold is great, from the peculiarity in the distribution of the blood, will have the heat of the system so much reduced, as to enfeeble or almost destroy, for the time, the organic functions. By lessening the temperature of an animal, we impair, or stop altogether, the digestive process; and, by the cessation of this, the numerous recrementitious changes are affected to a corresponding extent. But life can continue for a long time without the ordinary assistance of these functions. It exists when these are active, because there is an expenditure in the system equivalent to the contributions. If these were diminished, the excrementitious powers remaining the same, the constitution would soon perish. Therefore, life is maintained in a vigorous state by a balance of the corporeal faculties, whose office it is to assimilate what is nutritious to the system, or remove what is no longer required. As cold has the tendency to diminish the generation of animal heat, it circumscribesimmediately the desire of food and the power of digestion, and, independently of the direct influence which the latter exercises on other parts of the body, the capability or disposition to move is diminished in the same ratio by the agency of the same cause. In this view we have lessened the contributions and their expenditure; but as long as a uniformity of action subsists between them,

L 2 
we have no reason to suppose that life will be destroyed,-it will be prolonged according to the diminution of those powers which produce and support it.

CLXXXI. Cold having occasioned a certain sluggish state of the system, the animal instinctively retires from its severity, and fixes its abode for the winter in barns, caves, or holes in the ground. Before it takes up its retreat, the respiration, and the production of animal heat, have experienced greatalterations; and thesechangesin its secluded situation progressively increase with the intensity of the cold, till at last the former becomes almost imperceptible, or is performed at great intervals, and the latter is affected to the same extent. "Mr CoRNish applied a thermometer to a torpid bat, and found that it indicated $36^{\circ}$;" and Spallanzani found the chest of a bat, in similar circumstances, to have a temperature as low as $47^{\circ}$. We observe, in these instances, a great diminution of temperature, and this never occurs unless it has been preceded by a corresponding derangement of the respiratory function. At this period the senses are obliterated, the various instincts no longer exist, nor do the necessities of nature any longer prompt the animal to exertion; its secretions have ceased, nor is there any function in evident action that can materially tend to waste the strength of the body. We perceive the slow and almost imperceptible func- 
tion of respiration, and the weakened motions of the heart ; but these are amply sufficient to supply the demands of the system.

CLXXXII. The nature and influence of those changes which predispose to, or precede torpidity, are manifestly the depressing effects of cold, diminished temperature, and almost total extinction of every assimilating faculty. But it is impossible to give limits to life; it is not a simple power, but an aggregate of powers, the extent and energy of which may be lessened, in the same manner as we modify chemical attraction, by diluting the active materials that dispose themselves to sudden or violent combination; but, in this case, the laws which regulate the changes are unaltered,-the slowness or extent of the operation in the one does not destroy the character of attraction, nor does the diminution of organic laws in the animal and vegetable creation render the continuance of life impossible. Animal existence and attraction exhibit every gradation of force, from the zoophyte to man, from the tedious formation of a crystal to instantaneous explosion; and when cold, by determining the blood upon the internal viscera, lessens the generation of animal heat, in co-operation with its consequences, it enfeebles, to the same extent, the activity of animal and organic life.

CLXXXIII. No part of Nature subject to the laws of organization is indebted for its existence to 
the excited activity of its functions, but to the observance of those intimate relations subsisting between the powers which $a d d$ and those which subtract. Whenever the former predominate over the latter, life then displays its exuberance, the plant throws out its leaves or expands its flowers, and the energy of the animal frame is concentrated to strengthen old or develope new functions, or to excite disease; and when the latter become superior to the former, the plant droops, and the animal decays. But if these powers are equally diminished, as vegetation is in winter, or as happens to animals in a state of torpidity, it is almost impossible to prescribe boundaries to their duration.

CLXXXIV. Many theories have been proposed to explain the cause of torpidity. MANGILI imagined that the veins are larger, in pro. portion to the arteries, in hybernating than in other animals. "He supposes, in consequence of this arrangement, there is only as much blood transmitted to the brain during summer as is necessary to excite that organ to action. In winter, when the circulation is slow, the small quantity of blood transmitted to the brain is inadequate to produce the effect."* Pallas observed the thymous gland and two small glandular bodies under the throat

* The Philosophy of Zoology, by John Fleming, D. D. Vol. Il. p. 61 . 
and upper part of the thorax unusually large, florid, and vascular during torpidity. The opinion I have brought forward to account for the occurrence of the phenomenon, viz. that it depends on the character of the external circulation, the effects of which modify the production of animal heat, whose influence is felt whether excited or depressed, by every organ of the body, is consistent with a variety of facts and analogies, and in harmony with every appearance which these naturalists have adduced in support of their own view.

CLXXXV. The enlarged glands, their florid and vascular aspect, the seeming superiority of the veins over the arteries, are the very consequences we should expect from the altered distribution of the blood. If these had not been present, the explanation I have given would have been founded on assumed premises, and unsupported by legitimate deductions. As the severity of the cold increases, the circulation is changed; its vigour and general diffusion become now feeble and concentrated; the blood, which previously warmed the whole system, is now bestowed upon the internal organs, debilitating their functions, as is proved by the diminution of temperature, and the weak and slow pulsations of the heart : but the determination is not confined to these vital organs, but spreads itself throughout the internal parts, producing congestion, or, in other 
words, enlarged glands, swollen veins, and a florid and vascular appearance of almost every viscus. The veins seem to be proportionally larger than the arteries, because the blood can sojourn in them with greater facility than in the arteries, in which motion is communicated to the blood, by the heart, at all times sufficiently strong to make the accumulation less in the one than the other; and it was simply from this reason that Mangili observed the veins larger than the arteries, and, from this and other causes explained, that Pallas had remarked the glandular bodies of the throat more prominent and vascular than usual.

CLXXXVI. The state of the system during repose is evidently not mere slumber, as has been supposed, for this does not retard the organic functions, but is an extinction of ninetenths of those powers that constitute its active life, and the fraction which remains is not occupied, as in health, in the assimilation of food, on in attending to the multifarious secretory and absorbent actions, but is sluggishly engaged in the performance of respiration and circulation. The former is employed in oxygenating the blood, and in removing from the body certain excrementitious matters, the product of the vital action still going on ; and the latter is feebly contracting in order to propel the slender stream of blood to the various parts of the system, In fine, 
the office of both is to maintain as exact as possible the balance between the powers which add and those which subtract. From the reak propelling force of the heart, and the agency of the external cold, the energy of circulation and the quantity of blood will necessarily diminish, as we recede from the heart to the surface of the body. If the wants of the system were not regulated according to these conditions, the function of respiration and the motion of the heart would be inadequate to preserve in existence the collective instruments of life. By circumscribing the extent of circulation by means of ligatures, as was proved by Legali.ors, or by lessening the demands of the constitution, as in torpidity, by the successive reduction of the energy of circulation, and the quantity of blood from the heart to the surface of the body, we enable the heart to contract, and the lungs to improve the qualities of the sanguineous fluid. The experiments of LEGaLLOIS demonstrate the correctness of what is advanced. “ Si avant de détruire la moëlle on fait des ligatures, soit à l'aorte, soit à quelques gros trones arteriels, les resultats sont differens, et la destruction de la même portion de moëlle, qui, sans ces ligatures, eut arrêté subitement la circulation, sera insuffisante pour produire cet effet. En général, en resserant par des ligatures l'etendue des parties auxquelles le cœur doit distribuer le sang, on diminue la somme des forces dont cet organe a besoin pour remplir sa function, et l'on 
racourcit à mesure la longeur de la moëlle indispensable pour l'entretien de la circulation."*

CLXXXVII. During the continuance of the lethargy the animal loses much in weight. The experiments of Dr Monno on the hedge-hog, of MANGili on marmots, and those of $\mathrm{Mr}$ CorNIsH on bats and dormice, are sufficient to confirm the truth of the statement. It is scarcely possible to conceive that respiration should act, even feebly, for several months, without affecting the weight of the body to the extent observed. It is remarked by MangiLI, that this circumstance becomes more obvious if the animal has been roused several times from its torpid state; and this is what we ought to expect if we consider that, every time the animal is revived, the functions of the lungs and heart are quickened,thus diffusing a greater stimulus throughout the system, improving the action of the absorbents and secernents, voithout any food being taken to supply these additional demands. The animal returns to its lethargic condition; but it is probable that several days transpire before the necessary organic relations are re-established, and, until this takes place, the powers appropriated to subtract exceed those which add, and thus it is easy to account for the diminished weight from repeated reviviscences.

* Experiences sur le Principe de la Vie. Par M. LEGALLOIS, M. D. p. 144. 
CLXXXVIII. As the nature and influence of those changes which predispose to or precede torpidity were found to be the application of cold and the diminution of temperature, the nature and influence of those changes which predispose to, or precede the return to active life, will consequently be found to consist in the application of warmth and the increase of temperature.

CLXXXIX. It is not improbable that the system thus emaciated by the evolution of unessential matters from the lungs, may become more susceptible to external agents, or uneasy sensations may be excited, the tendency of which is to accelerate the respiration by the slight degrees of muscular exercise occasioned. But not wishing to indulge in hypotheses, I shall leave the consideration of this point to others.

CXC. In the months of March and April, the greater number of hibernating animals revive; and it is at this season of the year that vegetation shows symptoms of returning vigour, from themilder state of the atmosphere and the influence of the solar rays. It is imagined by some, that the barns, caves, and holes in the ground, to which these animals have retired, prevent them from feeling the beneficial agency of spring. Dr Fleming says, "It cannot be owing to any considerable change of temperature; for their situation prevents them from experiencing such vicissitudes."*

* Vol. 1I. p. 69. 
There is no place to which these animals retreat that is exempt from the great vicissitudes of seasons. If they burrow in the ground, the severity of the cold is softened by their numbers ; but if the degree of cold becomes less intense by $10^{\circ}$ or $20^{\circ}$, the warm atmosphere which they have created will become warmer by the circulation of more temperate air.

CXCI. As the medium to which they are subjected gradually improves in temperature, the blood that was determined to the internal organs is, by the most imperceptible steps, recalled to the surface. The lungs are then enabled to produce a greater effect upon the diminished quantity of the blood; and as this is more stimulating to the heart, it is propelled with greater force, and, meeting with less resistance, on account of the additional warmth from without, it is distributed more equally throughout the system. While these minute changes are going on, the generation of animal heat is increased; so that the situation to which the animal is confined has its temperature augmented. The external heat continues to act upon the surface of the body and on the lungs, respiration becomes more frequent and complete, the blood circulates with greater energy, muscular exertion is excited, and these causes, calculated to promote the same object, are progressive in their influence; and the various powers act 
and react the same series of changes for an indefinite period, with greater facility and perfection each time, till at last the animal, stirred from within, and stimulated from without, is awakened to the possession and enjoyment of its animal and organic functions. 


\section{CHAP. VIII.}

The means by which the System is enabled to bear a Temperature much superior to that of the Body.

CXCII. From the preceding chapters the reader has probably anticipated the greater part of the views connected with the elucidation of the present; and, from the facility with which many of the principles can be applied, may think it unnecessary to enlarge further upon them : but, for so doing, I hope the importance of the investigation will be a sufficient apology. Different chemists and physiologists have entertained different opinions regarding the powers of the system in accommodating themselves to a high temperature. It is stated by Crawford, that the blood is less venalized in the capillary vessels when the body is subjected to great heat; and, therefore, the changes which this undergoes in the lungs are less extensive for want of the combination of the oxygen of the air with the usual quantity of inflammablematter contained in the venous blood. Dr Edwards of Paris endeavours to show, that less oxygen is consumed in summer than winter, and that the temperature of the system is, on that account, little higher than at the latter season. I have already shown that his experiments, 
although correct, do not in the least warrant the conclusions that he has deduced from them, as he has omitted to take into consideration the various conditions of the system at the different seasons and periods of life, which have been shown to modify the results of his experiments. It has been supposed by some, that the evaporation which takes place at high temperatures is the principal reason that the effects of heat are counteracted; by others, that the blood is cooled in its circulation from being distributed to parts possessing different degrees of heat.

CXCIII. It is impossible to deny the great influence which the two latter causes exert in reducing the temperature of the body when subjected to a heat a few degrees higher than what is natural ; but I cannot consider them sufficiently powerful to account for the possibility of bearing the degree of heat to which Fondyce, DEI.ARoche, and Berger have been exposed. Believing these causes to be insufficient, and at the same time being persuaded that the views of Crawrord are altogether objectionable, I shall attempt to give other reasons to explain the resources of the animal economy.

CXCIV. When an individual is engaged in violent exercise, the blood becomes more equally distributed throughout the system, and the tendency of this change is to relieve the lungs and internal viscera of a quantity of sanguineous 
fluid, thereby enabling the inspired air to oxy. genate more completely the diminished proportion which remains ; or, if he be exposed to external heat, precisely the same consequences follow. The surface of the body is stimulated and the blood flows to its minute vessels in greater quantity and velocity than usual ; and in this way the lungs and heart are quickly excited,-the result of which is that they co-operate with the external heat in maintaining the diffused condition of the circulation, and in promoting the same physical phenomena.

CXCV. If the external heat be very great, the same effects become more apparent; because the quantity of blood transmitted through the lungs at any one moment diminishes in the increasing ratio of the temperature. In XviIr. $\mathrm{xIx}$. and Xx. I explained why animal heat is not in the direct ratio to the quantity of oxygen inhaled, but in the inverse ratio to the quantity of blood exposed to this principle. In extending the same view, we shall understand why the system is capable of resisting a temperature of from $240^{\circ}$ to $260^{\circ}$, which was experienced by BLAGDEN.

CXCVI. After an ordinary expiration, the quantity of air remaining in the lungs is estimated at 280 or 290 cubic inches; but if the temperature of the body be increased from $5^{\circ}$ to $10^{\circ}$ more, the air will be much rarefied, and, consequently, the same quantity will not continue to act upon 
the blood. It will undergo this change before it is inspired, which will lessen the quantity re ceived, and the internal warmth of the chest will also tend to carry this change still farther. So we observe that two causes are in operation, both of which will diminish the usual proportion of air acting on the blood; and, moreover, this diminution will be in the direct ratio to the increase of temperature, retarding its influence, not by the generation of cold, but by setting limits to the generation of heat.

CXCVII. Crawford endeavoured to shew, by direct experiment, "that when an animal is placed in a heated medium, the sanguineous fluid during each revolution is less impregnated with the inflammable principle; for the venous blood, in these circumstances, becomes gradually paler in its colour, till at length it acquires nearly the appearance of the arterial." * The experiments which he instituted, for the purpose of proving this point, are far from being satisfac. tory.

An animal was confined in a vessel containing atmospheric air, which was at one time surrounded by water at $55^{\circ}$, for 42 minutes, and at another by water at $104^{\circ}$, for the same period. At the termination of the experiment, the air in the former was found to be only one-fourth as pure

\footnotetext{
* Crawropd, Experiments and Observations upon Animal Heat, \&c. p. $\$ 87$.
} 
as atmopheric air, while that in the latter was one-half. From these results he concluded, that less oxygen is consumed in a heated state of the system than when the medium is cold.

It is impossible to estimate the changes or conditions of the blood by any degrees of alterations which the inspired air undergoes. At one time, when the blood is determined to the internal viscera, by cold or disease, the proportion in the lungs may be calculated at 30 , while the natural quantity may be only 10 ; and if this should occur, the air will be vitiated to a greater extent in the former than in the latter case, without the same quantity of heat being produced. It must be obvious to the reflecting mind, that, if the quantity of blood in the lungs be increased threefold, without the surface which it offers to the air being enlarged to the same degree, that more oxygen may be consumed without the blood having derived the ordinary advantages from the contact. When the proportion of 10 presents its surface of blood to the action of the air, it is probable that this is almost as extensive as that of 30 ; the same capillary vessels are occupied by both ; and if they become congested by the addition of 10 or 20 , this very state of the vessels may prevent the usual complete changes of the blood. In support of this opinion I may observe, that in every instance in which the quantity of sanguineous fluid in the lungs is greater than what 
is natural, it is less perfectly arterialized; and whenever the contrary state is present, its qualities are improved.

CXCVIII. Crawford remarks, that, when an animal has been exposed for some time to a high temperature, the blood in the veins assumes the colour of that which circulates in the arteries. He explains this appearance, by stating, that " the sanguineous fluid is less impregnated with the inflammable principle," or, to employ terms more intelligible to modern readers, that the blond has not undergone its accustomed changes in the capillaries of the body, and therefore is less venalized. When the system is subjected to great heat, less blood at any one moment is transmitted through the lungs, the consequence of which is, that it is more perfectly arterialized, and, from the increased action of the heart, returns to the chest in about one half of the usual time. It is therefore manifest, that the blood is favourably circum. stanced for the operation of chemical changes; on the one hand its quantity is diminished, and on the other the repetition of these changes is doub. led. These reasons alone are sufficient to explain the altered colour of the blood.

CXCIX. As Crawrond had found this appearance in every instance in which an animal was submitted to warm air or water, he was desirous of trying the effects of cold air or water. For this purpose, a dog was immersed in watep 
at $45^{\circ}$, and in a quarter of an hour blood was drawn from the jugular vein, and was thought " to be the darkest venous blood we had ever seen." This result was considered to support the explanation, viz. that, when the temperature was high, the changes of the blood in the minute vessels were less perfect than usual; but when the temperature was low, these changes were supposed to be more complete, accounting for the very dark condition of the venous blood. The dog immersed in water at $45^{\circ}$ must have had its heat materially diminished, although this important point was not alluded to. The blood, from the medium in which the body was placed, would be determined upon the internal organs, which would tend to obstruct its proper oxygenation. It cannot, therefore, be denied, that the difference in colour between the arterial and venous fluid, in every state of the system, is to be attributed to. its more or less perfect alterations in the lungs.

CC. If the dog whose blood was very dark had had its temperature augmented, which it ought to have had according to his doctrine, there would then have been a plausibility in the theory; but it must have lost several degrees of heat, for the blood, in no condition of the system, assumes this aspect, unless the temperature be diminished, and congestion in some part of the body be present. To ascertain the temperature of

* Crawrord, p. 310 . 
the body when immersed in cold water, the following experiments were performed.

\section{EXPERIMENT I.}

A fine adult rabbit, of the temperature of $104^{\circ}$, was immersed, with the exception of the head, in water of $502^{\circ}$. In two or three minutes, the animal began to shiver; in five it was removed; and its temperature was found to be $94^{\circ}$. It was directly immersed in warm water of $106^{\circ}$ for the same space of time, during which it gained 4 degrees, its temperature being $98^{\circ}$.

\section{EXPERIMENT II.}

An adult rabbit, temperature $106^{\circ}$, but not so robust as the preceding, was immersed in water of $50 \frac{1}{2}^{\circ}$ for five minutes, during which time its temperature was reduced to 96 $\mathfrak{2}^{\circ}$ : It was immediately placed in warm water of $110^{\circ}$ for the same time, at the end of which its heat had risen to 98 ${ }_{4}^{\circ}$. We did not think it necessary to keep the rabbits a longer time in the water. They both seemed much oppressed : and when we consider that one, in the short space of five minutes, lost $10^{\circ}$, and the other about $8^{\circ}$, we have changes sufficiently great to account for such symptoms.

CCI. Without the corroboration of the preceding experiments, the description which Crawford himself gives of the appearance of one animal subjected to a cold medium, and of another to a warm one, is sufficient to prove the consistency of the present opinion. The one exposed to cold 
showed symptoms of great uneasiness: " the eye in particular seemed to be much swollen, and became dim and languid;" but the eye of the other dog, exposed to warmth, retained its brightness to the last." Whenever the blood is properly, or unusually oxygenated, the expression of the eye is such as he describes; but when it is deficient in its ordinary qualities, it then becomes swollen, dim, and languid: it is swollen, because its vessels are congested, and dim and languid on account of this condition and the deteriorated properties of the blood.

In all the rabbits to which $I$ have given opium, the eye exhibited the appearances $I$ have stated; and in these the temperature was lessened $7^{\circ}$ or $8^{\circ}$, and the distribution of the blood was shewn to have been unequal by the different appearances observed on dissection.

CCII. It is remarked by CrAwFORD, that "The cold stage of fevers is preceded by languor, a sense of debility, and a diminution in the action of the heart and arteries. The respiration is small, the pulse is weaker than natural,-the quantity of blood which passes through the lungs in a given time is diminished,-and hence less phlogiston will be discharged from the blood, and, consequently, less heat will be separated from the air."†

* Crawford, p. $313 .+1 b i d$. p. 394. 
In the cold stage of fevers the surface of the body is collapsed and pale, and the blood is evidently concentrated in the internal organs contained in the three great cavities of the body. The difficulty of breathing, and oppression in the chest, shew that the lungs and heart are implicated ; the pallid aspect of the face, the dulness of the eye, and the pain of the head, or occasional delirium, prove the brain to be affected, and the enlarged liver and spleen demonstrate a similar condition of the abdominal viscera. Since the lungs have more blood than is natural, it is manifest that his idea of a diminished quantity passing through them has little relation to the quantity on which the air has to act; and as this is augmented, the consequences are in harmony with the principles stated in the first chapter, viz. That animal heat is not in the direct ratio of the quantity of oxygen inhaled, but in the inverse ratio of the quantity of blood exposed to this principle.

CCIII. It is stated by CrawFord, that the capacity of the arterial blood for caloric is greater than that of the venous; and the opinion beautifully illustrates his ideas of animal heat, as he imagined this to be extricated in the minute capillary vessels of the system, and not in the lungs; and it also removes the difficulties which were opposed to the views of Dr BLAcK, which supposed the evolution of this principle to take place in the lungs. This important point has been inves- 
tigated by several distinguished physiologists since the time of CRAWForD, and it seems now to be generally believed, that the arterial has a temperature of one or two degrees superior to that of the venous blood. This opinion militates very much against the one brought forward by CrawFORD, and may be said to destroy the consistency of his theory.

CCIV. The body was supposed, by CRAwFord, to possess a uniformity of temperature at the various seasons of the year, and throughout the whole system. The former condition was regarded as arising from the less perfect changes of the vital fluid in the capillary vessels, in consequence of which the quantity of inflammable matter brought by the veins to the lungs was diminished; and as he believed the evolution of animal heat to be proportionate to the chemical changes between the inspired air and this inflammable matter, it followed, from his doctrine, that less heat would be generated in summer, when the necessities were less, and more in winter, when they are greater. In cxcviri. and cxcIx. I have shewn the fallacy of those premises by which he was led to draw this conclusion. The equality of temperature throughout the system naturally flowed from his principles, which supposed animal heat to be given out by the blood in imbibing the phlogistic principle from the capillaries. 
If a thermometer be applied in winter to different parts of the body, it will indicate different degrees of temperature ; and, generally speaking, all much less than that of the internal cavities, as proved by the instrument being placed beneath the tongue. In summer there will be a greater equality, because the sanguineous fluid is warmer by one or two degrees ;* it also circulates with greater velocity, and is more generally diffused; and, independently of these considerations, the atmosphere is more temperate, tending rather to court than repel its equable distribution.

CCV. When the body is the warmest, the blood cannot be cooled to any great extent in the course of its circulation; and, therefore, when the system has a demand for its various resources to overcome the excessive heat to which it is exposed, the distribution of the blood cannot be regarded as a very efficient means, as supposed by Dr Monro, Secundus, of Edinburgh, and Mr BeLL of Manchester.

CCVI. The evaporation from the lungs and surface of the body is the most powerful agent that physiologists have alluded to, in controlling the temperature of the system; its beneficial effects are experienced in inflammatory fevers, and it is often promoted in diseases, in order to equalize the circulation and reduce the temperature; but its office is to remove the heat already

* Vid. Edwards, p. 489. 
existing, and not to set limits to its production; it is an effect which tends to preserve the constitution, but is not the cause that regulates the generation of animal heat.

CCVII. When an individual has been using violent exercise for some time, perspiration flows profusely from the body. Is this to diminish the heat, or to retard its generation? Or, if we suppose the same to be exposed to a medium of $100^{\circ}$, an evaporation from the surface takes place proportionate to the external temperature; if the medium become $150^{\circ}$, evaporation continues to correspond; if that be raised to $200^{\circ}$ or $250^{\circ}$, the body is literally suffused with moisture. Since, in the first instance, perspiration was the consequence of increased heat, without having the least influence on the source which produces it, for it continues to flow as long as the muscular exertion remains, and ceases as soon as this subsides, or as soon as the system recovers its regular temperature, it cannot be regarded as a cause that regulates the production of heat.*

CCVIII. The profuse quantity of perspiration does not affect the reasoning,-for this is merely in accordance with the excessive heat of the body. If we could, by exercise, produce as great a temperature as is occasioned by the external medium, we should observe the same results; but in this case we could not say that the

* Dr Edwards, pp. 254, 488. 
perspiration had protected the system from the further production of heat: we might with truth assert, that it had preserved it from its injurious effects. If an individual be confined in a warm moist atmosphere, or in heated water, he is very soon oppressed, because perspiration is retarded in the one case and prevented in the other, and consequently the heat accumulates.

CCIX. There is a great difference between the powers that relieve the system from the effects of heat and those that circumscribe its evolution. The former are simply the extension of the ordinary and obvious laws of the animal economy; the latter are the result of a principle less evident, and exercised only on extraordinary occasions. The excessive heat of $240^{\circ}$ or $260^{\circ}$ would destroy the body, if the $260^{\circ}$ increased the generation of heat in the ratio of its numerical progression; but every degree, from the temperature of the body to $260^{\circ}$, modifies the production of heat by augmenting the rarefaction of the air within the chest, - thus successively diminishing the quantity of oxygen submitted to the influence of the blood.

When an individual has been exposed to great heat for some time, as in an oven for example, we observe, that, on quitting this, his breathing is quick and short, presenting symptoms somewhat similar to those occasioned by running, or excessive exercise. When such arise from the latter causes, 
they have been stated in XxI. as originating from the diminished quantity of air within the chest; and it appears equally consistent to refer those co-existent with great heat to the same physical principle. If, therefore, the air is rarefied before it is inspired, and if its general bulk be subsequently materially lessened; and if, in conjunction with these two conditions, we consider, that the quantily of air in the lungs indispensable to maintain the powers of life is very much rarefied by an extensive and constant evolution of caloric, we are enabled to appreciate the boundaries which Nature has set to the generation of animal heat. 


\section{CHAP. IX.}

The Influence of Disease on the Production of Heat.

CCX. To do justice to this subject would require more time than $I$ can at present devote to it, and more correct and multiplied data than the records of medical science furnish. The various states of the pulse, the appearance and quantity of the excretions, the expression of the countenance, the colour of the skin, the operation of the mental powers, and many other conditions of the system, have been anxiously inquired after and carefully studied when symptoms of disease were urgent, while the changes in the temperature of the body, or in the distribution of the blood, have never been objects of minute consideration.

CCXI. We cannot expect to derive much exact knowledge from the ancients; their ideas concerning the source of heat were crude and fallacious, and their acquaintance with the most important laws of the animal economy, or of the obvious connections of the different organs, or of the degrees of influence which one part of the body exercises over another, was necessarily bounded or empirical. If we turn to the moderns, we have abundance of valuable facts in almost 
every department of medical science, except on the subject of animal heat in relation to disease. While chemists have been contending about the quantity of oxygen consumed in a given time, or about the proportions of carbonic acid and vapour formed by the changes in the lungs: physiologists have been equally busy, but less beneficially occupied, in endeavouring to prove the efficiency of the nervous system in the generation of heat,-in attempting to show that galvanism and the nervous fluid are the same-or in labouring to demonstrate that animal heat is nothing but a secretion. If these opinions were to be tested by their contributions to practice, or by the extent of their suggestions to the same, I fear they would be found like many other views, whose only support is the ingenuity of their authors.

CCXII. To these causes we are to attribute the routine practice of the profession-the adherence to what is old, or the avidity for what is new.

CCXIII. If we take into consideration that the greater number of diseases arise from causes that over-excite the respiratory and sanguiferous functions, or from causes that diminish their energy, it is certainly important to know in what way these agents influence the system. In all local and acute inflammations the temperature is augmented, the pulse is more frequent, full, or bounding, the sensorial powers are more or less disturbed, and the secretions are also 
irregular. The degree of temperature is regulated by the situation of the organ affected, or by the disorders which this has communicated to the lungs and heart. If, for example, any of the viscera of the thorax be the seat of inflammation, we have then the flushed or livid countenance, according to the stage or severity of the affection,-the pulse is, for the most part, hard and frequent, and a variety of other symptoms are present equally characteristic of the local disease. The increased frequency of respiration, and the preponderance of its expiratory character, are to be regarded as the principal causes in augmenting the evolution of heat. Before disorganizations occur, the blood circulates with greater velocity through the lungs; the constant or frequent cough prevents or retards its occasional accumulation; and, from these conditions, the sanguineous fluid is more completely oxygenated, and, having acquired properlies more stimulating than usual, it excites palpitation or violent action of the heart. It is at this time that the blood is diffused generally throughout the body.

The temperature and pulse assume their usual characters as the organs regain their ordinary state; but if, instead of this favourable termination, the disease lose its inflammatory nature, and exhibit asthenic symptoms, we observe the pale and col. lapsed countenance, cold extremities, the surface pallid and shrunk, the patient troubled with 
shiverings, the pulse small and frequent, and the generation of animal heat controlled.

CCXIV. As the efforts of the system were unable to overcome the symptoms by which it was oppressed, the lungs would gradually become congested, thereby losing the power of maintaining the ordinary stimulating qualities of the blood; and as this would be insufficient to excite the usual contractions of the heart, the pulsations would necessarily become feeble, and frequent, because it is compelled to circulate what it receives. The propelling energies being lessened, the vital fluid is not transmitted to the distant parts of the body with its accustomed force, but the veins possess their usual disposition to return their contents; the consequence of which is, that the viscera of every cavity have their functions disordered by the unusual determination of venous, and the deteriorated properties of the arterial blood.

CCXV. If we suppose the intestines to be the seat of acute inflammation, the temperature is augmented, because the respiratory functions are again disordered. This effect is not to be referred to any nervous connection between the thoracic and abdominal viscera, but to the changes in the distribution of the blood. We can stimulate the action of the lungs by warming the inferior extremities, or we can oppress it by cooling the same; and, if alterations so far removed 
from the chest have an almost immediate influence, we can scarcely deny that a similar consequence will follow if the abdomen be excited or depressed by disease. Respiration in these eases is generally performed by the thorax, and the patient is extremely careful not to draw deep inspirations in which the abdominal muscles are concerned, from the pain which accompanies the attempt.

The pulse in this affection is small and frequent, sometimes hard; and these are the conditions we should expect from a knowledge of the nature and situation of the disease. The lungs are stimulated to an unusual action, but to a less extent than when their own tissue is exclusively implicated, and therefore the sanguineous fluid is not so well adapted, from its changes, to present the strong and bounding pulse. In accordance with this opinion, it may be observed, that inflammation of the abdominal viscera seldom or never affords the buffy coat equally well marked with that occurring in the thoracic.

CCXVI. If the symptoms tend to an unfavourable termination the temperature is lessened, and we have consequences similar to those men. tioned in ccxiv. 'The pulse is at times too frequent to count, or too feeble to be felt. If we consider the various important organs belonging to the abdomen, and the nature and activity of their functions, it will at once be apparent, whenever 
the blood has the tendency to accumulate here, that an inordinate quantity will very readily be determined to its different viscera ; and it is impossible for this state to exist without the lungs participating in the congestion : if these are oppressed, it is not difficult to explain the small, or almost imperceptible pulse, and other concomitant phenomena.

CCXVII. When the system is first affected by local inflammation, the increased temperature is to be attributed to the excited action of the lungs; a portion of the heat passes off in the form of perspiration, thus removing the superabundance which is evolved by disease. But if, instead of local and acute inflammation, we have other disordered conditions, giving rise to continued or intermittent fever, the distribution of the blood, and the temperature of the body, are accompanied by other and more marked changes.

CCXVIII. In the cold stage of intermittent fever the pulse is remarkably small and frequent, and in no other disease have we such an obvious depression of the powers of organic and animal life. Great diminution of temperature, difficulty of breathing, oppression of the chest, violent headache, pain in the course of the spine, and a general feeling of lassitude, are a few of the symptoms which characterize its development.

Dr Currie, in his Medical Reports, allows us to speak with confidence concerning the diminu- 
tion of temperature in the cold stage of intermittent fever. He says, "In the cold stage, the heat is diminished, not on the surface only, as some have imagined, but very probably over the whole system. I have found it under the tongue, and at the axilla, as low as $94^{\circ}, 93^{\circ}$, and $92^{\circ}$, and on the extremities many degrees lower."*

CCXIX. No state of the system more satisfactorily shews, that animal heat is not in the direct ratio of the quantity of oxygen inhaled, but in the inverse ratio of the quantity of blood exposed to this principle, than intermittent fever. The san. guineous fluid, from causes unknown to us, leaves the surface and extremities, or rather does not flow to them, and gradually engorges the internal viscera; the lungs for some time are less congested than the rest from the character of their office, but they are sufficiently influenced from the first to be incapable of oxygenating the blood as in health; and as their incapability augments with the continuance of the cause, they very soon co-operate with the depressing effects of the disease, to impede, if not to destroy, the exercise of their own and every other function of the body. In this way we can account for the sinall and frequent pulse andall the other symptoms enumerated. The blood is deficient in its usual properties, and there.

* Medical Reports, p. 153.

N 2 
fore cannot be expected to excite the contractions of the heart; and as it is not propelled throughout the arterial system with regularity or force, the remote arteries, from the want of the ordinary vis a tergo, and the accustomed quantity and qualities of the vital fluid, to stimulate these powers, on which their pulsations depend, exhibit a feeble or almost imperceptible action.

CCXX. When these symptoms cannot proceed any farther without endangering life, they are by degrees mitigated by the reaction of the system. It was shown by Huvter, when an animal is cooled down to a point much lower than what is natural, that the intense cold is counteracted by the evolution of a greater quantity of heat; and it is also stated by $\mathrm{Dr}$ EDWARDS, who has paid considerable attention to this subject, that it arises from the exertions of the animal to escape or remove itself from the depressing agent, thereby exciting the respiratory functions to the more perfect oxygenation of the blood; and it is to a similar cause, conjoined with the means we employ to promote the same result, that we are to attribute the return of the system to its normal state. In support of the general view brought forward, I may allude to the success of depletion in the cold stage of intermittent, as fully proved by $\mathrm{Dr}$ MAOKINTOSH of Edin burgh, in a variety of cases. 
CCXXI. I now proceed to show, that the heat of the body, in different stages of disease, is not a certain indication of the extent to which it is generated. When the system has been violently exercised or exposed to great heat, the increased quantity of this principleproduced is invariably dissipated by cutaneous perspiration and pulmonary evaporation. The reason of this is evident, the capillaries are in a healthy condition; and, whenever they are excited under these circumstances, they readily discharge the superabundance of caloric, and prevent the system from being oppressed by its accumulation. When the body is suffering from continued fever, the truly inflammatory symptoms have subsided ; in other words, the excited action of diseased organs no longer stimulates the respiratory functions to increased energy. From the slowness of the respirations, or their incom. pleteness, the inactivity of the patient, the paucity and derangement of the secretions, the almost perfect repose of the assimilating functions, the disturbance of the intellectual faculties, and from other co-existing disorders, it is manifest that less heat at this time is generated, and yet the temperature of the body is at times several degrees above its natural standard.

CCXXII. If we take into consideration that the uniformity of animal temperature is maintained by the balance of two opposite powers, viz. 
those that produce, and those those that expend, we shall find an easy solution of the seeming difficulty.

From the irregular and imperfect manner in which the vital fluid has for some timebeen prepared for the purposes of the system, every function is more or less disordered; and, among the rest that of the capillaries belonging to the surface of the body. These have not the same capability as formerly of evolving caloric : the consequence is, that even a diminished production of heat appears to present an augmentation, because the powers that expend are greatly inferior to those that produce. The truth of this may be observed in the dry and burning state of the skin. Dr Currie, in his Medical Reports, gives several remarkable cases of this condition of the surface, attended by restlessness and great fever, that was almost immediately removed by affusion or sponging of the body, and followed by the reestablishment of health, or the amelioration of the general symptoms. The benefit is not to be referred to the lessened generation of animal heat, but to its lessened accumulation.

CCXXIII. If we believe that every assimilative process is accompanied by the disengagement of heat, which is certainly a plausible opinion, but of which there is no direct proof, it must be considered extraordinary, unless the above view be received as correct, that the system should, in such a case, possess a temperature much higher 
than ordinary. We have many symptoms present which clearly show that the lungs are congested, and are otherwise very unfavourably circumstanced for the production of animal heat.

CCXXIV. The great heat of the surface has induced many practitioners to try depletion at a period of the fever much later than it is generally recommended and found beneficial. When attending, as a student, the Dispensary of $\mathrm{Dr}$ MACKIntosh of Edinburgh, I had an opportunity of seeing a great deal of continued fever, and I remember one case which will enable me to illustrate the present subject. I was called to visit a boy who had been ill for two or three days, and found that he was affected with the fever at that time prevalent in the city. Eight or ten ounces of blood were abstracted ; purgatives, aperients, and blisters were employed till the eleventh or twelfth day, according to the urgency of the symptoms. At this time his respiration became so difficult, accompanied with a fixed pain in the chest, and with great dryness and heat of the surface of the body, that I thought it advisable to bleed. Eight ounces of blood were drawn, with slight relief to the breathing; but the depletion was succeeded, in less than a quarter of an hour, by subsultus tendinum. The boy ultimately recovered, but the unfavourable symptoms developed on the second depletion warned me of the danger of bleeding, however the symp- 
toms may appear, unless such a measure should be warranted by other signs than simply increased heat of surface. The blood did not exhibit the buffy coat.

CCXXV. It is often remarked, that feverpatients are occasionally highly susceptible to cold when the temperature of the body is probably $104^{\circ}$ or $105^{\circ}$. Dr CurRIE relates a case where it was $109^{\circ}$, and yet the individual complained on the least exposure to cold. Whenever this occurs, we ought to consider the whole or the greater part of this additional heat as the effect of diminished evaporation and not increased generation; and, if this be allowed, it is easy to explain the great sensibility of the patient.

The heat of the surface not being derived from the augmented action of the heart and lungs, but depending exclusively on the diseased state of the capillaries of the skin, it is manifest that the external air will speedily lessen the temperature, as it is not supported either by great energy of circulation, or unusually stimulating qualities of the blood. I cannot believe that a person whose temperature is several degrees above the ordinary standard will be particularly liable to feel the immediate influence of cold, if such be the consequence of, and maintained by, the vigorous action of the lungs and heart. The Russian, after having bathed himself for some time in the hot bath, delights to run among the snow, and does 
not experience any unpleasant sensation; but his system is not circumstanced as in continued fever. The circulation on the surface of the body is, from the first immersion, excited; and from the vascularity which succeeds, the lungs, in common with other internal organs, have a smaller quantity of blood to oxygenate, agreeably to the principles laid down in the first chapter; and, therefore, the blood becomes more stimulating, increasing the action of the heart, and of the whole of the arterial system, and consequently protects the surface from the agency of cold. Dr Currie observes that, when the system possesses a temperature of $102^{\circ}, 103^{\circ}, 104^{\circ}$, or $105^{\circ}$ in the hot stage of intermittent fever, " the sensation of heat is now strong and steady, and the accession of external air does not produce a return of chillness as before." * In further corroboration of this opinion, we do not find that individuals labouring under thoracic inflammations are as susceptible to this agent as when confined with continued fever. In the former circumstances, the greater part of the heat is attributable to augmented production, which places the system in a situation somewhat similar to that of the Russian : but, in the latter, the temperature may be said to be truly superficial, as it is not generated by superior powers, nor maintained by the effects which they produce.

* Medical Reports, p. 155. 
CCXXVI. The views contained in the present chapter, in regard to practice, are of vital importance. They disclose principles which explain the difference between great heat that is produced by the lungs, and the accumulation of the same arising from diminished evaporation; and this knowledge at once suggests a mode of treatment different from that pursued in many cases of fever, and which is not derived from empiricism, but from more extended acquaintance with the laws of the animal economy. The application of these views to practice will be deferred till inflammation and fevers be considered in a subsequent treatise. I may, at present, generally allude to a few of the characters which distinguish the production of great heat from its accumulation.

1. Whenever the pulse is, for the most part, hard, strong, and frequent, the respirations more numerous than natural, the heat of the skin greater than usual, and the temperature of the body above the ordinary standard, we may consider the additional heat as the consequence of increased production.

2. When the above states are modified in degree, accompanied by a burning skin, and great restlessness of mind, the augmentation of temperature is to be referred to increased generation and diminished evaporation.

3. When fever has continued for some time, until the secretions are evidently much disordered, and the surface of the body shews strong marks of asthenic 
disease, the inordinate temperature sometimes felt in these conditions is to be ascribed solely to diminished evaporation.

4. Blood drawn during increased production almost always exhibits the buffy coat, whereas the same abstracted in purely typhoid affections is seldom or never present, although the system be apparently oppressed by great heat. It follows also, where the production is slightly greater than natural, that the buffy coat will be proportionate to the influence of this cause.

5. Whenever the skin is dry and hot, we may consider the functions of the capillary vessels to be much deranged.

6. When the patient shows acute susceptibility to cold, how great soever the temperature may be, we may justly suspect diminished evaporation.

7. When the temperature is high, and the individual is not particularly sensible to cold, we niay conclude that the generation of caloric is augmented.

8. The arteries, in continued fever, in different parts of the body, occasionally pulsate much more strongly than natural, even when the truly inflammatory character of the disease is past; but such a symptom must not lead the practitioner to suppose that this, in conjunction with increased temperature from diminished evaporation, indicates the necessity of depletion. Such a phenomenon frequently occurs in dysentery and other diseases characterised by internal congestion. It does not arise from conditions that attend the ordinary excited states of the arteries, viz. the more stimulating properties of the blood and its greater propulsion, but from obstacles to its free circulation. 
CCXXVII. Many fevers have undoubtedly been cut short by the timely affusion of cold water, depletion, emetics, or by sudorifics ; and when such instances occur, it is not improbable that the quick pulse, pain of the head, restlessness, great heat of body, and other febrile symptoms, were not occasioned by inflammation or internal congestions, but by a morbid condition of the skin, retarding the ordinary evaporation from the surface. If other causes be assigned for the origin of such fevers, it is difficult to conceive how the employment of such means can act so immediately as to remove them. In former times, when the crisis of disease were much attended to, profuse perspiration, or moisture of the skin, was considered to augur a favourable termination of fever. The physician, in regarding this circumstance as favourable, was correct ; but he was led to his conclusions by experience alone. If we reflect that the high temperature of the body was, throughout the greater part of the disease, the consequence of diminished evaporation, exciting and maintaining disorders in every part of the system, it is manifest that the return of the capillaries to their usual function is a symptom we cannot too highly estimate.

CCXXVIII. If the whole of the system can become deranged from a general diminution of evaporation, giving rise to increase of temperature, the same effect may take place in any single part 
of the body. Phlegmonous inflammations are sometimes accompanied by great heat of their individual surface. If the temperature should rise above the standard of the system, a point which is yet disputed, I do not see that we are authorized to suppose that the additional degree is to be attributed to a power of generation belonging to the blood-vessels of the part, but, in a great measure, to diminished evaporation.

In physics, accelerated motion produces heat; but there is a great difference between animate and inanimate particles and the laws which respectively regulate them.

We are frequently justified, from obvious resemblance, in applying the same general reasoning to both, but we often err in endeavouring to draw analogies where no similarity exists.

CCXXIX. There is certainly a difference in the results between diminished evaporation affecting the whole surface of the body, and a single part. In the former instance, the whole circulating fluid becomes deranged in its quality and motion ; in the latter, its own vessels, of what kind soever, are, generally speaking, principally if not exclusively disordered. If symptomatic fever occur in these cases, it is as easily explained on the supposition of diminished evaporation as of increased generation of heat. In both cases the temperature of the vital fluid is augmented, and this condition of the blood is communicated 
to the heart and lungs, not by means of the nerves, but by the circulating medium. These organs being excited to greater action, produce the same general effects as if they had been primarily affected by any other stimulating agent.

CCXXX. Capillary circulation in the state of health may be considered independent of any power derived from the heart or arterial system, but, in the state of disease, still farther removed in its relations. The deranged action of any portion of its own system destroys the harmonious connection which unites it to the general system ; and, therefore, it is reasonable to expect, if a part of the body be thus circumstanced, that its surface will be incapable of performing the function of evaporation, and, consequently, that heat will be accumulated. Emollient cataplasms and evaporating lotions are among our most efficient means in cases of phlegmonous inflammations, where there are great heat and pain ; and it is not improbable that these are in part beneficial, because they reduce the accumulated heat, or promote the proper action of the capillaries subservient to evaporation. The apparent increase of heat in a local, superficial inflamma. tion, is not attributable to one cause, but to three :

1st, Diminished evaporation; $2 d$, Augmented quantity of blood in the part; and, $3 d$, The more 
frequent renewal of this fluid from the excited action of the capillaries.

If a given quantity of blood be transmitted to any part of the external surface of the body, the region in which it is distributed will feel warm; and if this quantity be doubled, without in the least affecting its temperature, the sensation of heat will be proportionally augmented, because a greater number of points, which communicate the sensation, is contained within a given space. If the blood which circulates on the surface of the body be renewed more frequently than natural, the part thus circumstanced will be warmer. The blood on the surface is seldom or never so warm as that which belongs to the cavities ; its temperature is diminished from the influence of an external medium, to which the internal circulation is not at all exposed; but when the vital fluid returns to the surface in one half of its usual time, the causes which tend to lessen its heat produce less decided effects, from its increased velocity, or diminished sojourn in the capillaries. 


\section{CHAP. X.}

\section{The function of the Eighth Pair of Nerves.}

CCXXXI. Ir is scarcely necessary to perform new experiments on the present subject to be enabled to speak with confidence regarding the phenomena connected with the destruction of the eighth pair of nerves. We have a sufficiency of examples illustrating the various changes which occur in the thoracic and abdominal viscera, when this part of the nervous system is injured; but although the general consequences, with few exceptions, are the same in the different experiments brought forward by physiologists, still we observe great discrepancies in the reasoning of those who attempt to explain the precise origin and character of these consequences.

CCXXXII. Before I adduce any new arguments in regard to the eighth pair of nerves, it will be advisable to take a glance at the nervous system in general. This part of the animal economy has been investigated with more judgment and success within the last few years than any other belonging to the system. Direct experiments, multiplied and varied in every possible way, have been appealed to in support of new 
discoveries, or in refutation of old opinions : thus on the one hand presenting knowledge as an immoveable point for future advancement, and, on the other, correcting the fallacies of ignorance. But while the present age has been enriched with contributions of this kind by Bichat, GaLL, MAGENDIE, LEGALLOIS, and BELL, it is also distinguished by theories and hypotheses, which are probably almost inseparable from the preceding. The development of one fact in physiology, too generally gives rise to a multitude of ingenious ideas, which seem to be required to complete the imperfect view which it unfolds; and it is from this cause that every century is necessitated to retrace its steps to some established truth that has been lost in the refinements or visions of its admirers._" Eh bien! la physiologie a encore un pied dans la première époque, tandisque déjà elle en a placé un dans la seconde. C'est aux physiologistes actuels à lui faire le pas tout entier."

CCXXXIII. So natural is it in man to embellish what he cultivates, or to colour with his pursuits every object which he studies, that we have now cerebral phenomena explained on the supposed oscillations of the nervous fluid; the inflammatory condition of the blood, from the interposition of this same agent between its globules; $*$ hypochondriacal and melancholic de-

* Nequaquam, sed sanguis, cui nullum additamentum accessit, supplementum nullum a nutritionis foco proficiscens, dilatatior, expanditur majorem acquirit capacitatem pro principio nervoso ipsius globulis interposito.-LOBSTEIN, p. 119. 
rangements, nausea, want of appetite and palpitation of the heart, from the diseased state of the nerves; and, lastly, the generation of animal heat is attributed to secretion, and all secretions are referred to the influence of the nerves.

CCXXXIV. Some of these opinions have been transmitted, with the diseases they are imagined to explain, from a remote period; and they might have continued for as long a time to perplex the scientific physician, if important discoveries had not allowed us to examine them with greater philosophical acuteness. If our knowledge had been confined to the nerves appropriated to the senses or to motion, it would have been impossible to deny, that, as certain parts of the nervous system have obvious functions to perform, it is not improbable that others, still unknown, may possess the faculty of secreting the various fluids of the body, but the discovery of Mr Charles Bell has thrown considerable light on this subject. He proves that " the anterior column of each lateral division of the spinal marrow is for motion, the posterior column is for sensation, and the middle one is for respiration ;"* and, still further, "that there is an obvious division of the medulla spinalis, corresponding to the cerebrum and cerebellum; every regular nerve has two roots, one from the anterior of these columns, the other from the posterior: such are the fifth pair, the sub-occipital, the

* Bell on the Nerves, p. 22. 
seven cervical, the twelve dorsal, the five lumbar, and the six sacral. They are common to all animals, from the worm up to man, and are for the purposes of common sensation and motion, or acts of volition."* This discovery enables us to cultivate an intimate acquaintance with the constitution of the nerves belonging to the thoracic and abdominal cavities, - to note with precision their origin, their compound nature, and to understand the character of those endowments which they bestow on the different viscera on which they are distributed.

CCXXXV. If we believe, with some anatomists, that the sympathetic nerve is derived from the fifth and sixth, we shall be enabled to speak with confidence of its properties ; or if we grant that it is merely connected with these nerves, we are still able to discriminate its functions, by observing the office of those organs and muscles to which it is distributed, and by noting the consequences which follow its partial destruction, or the phenomena which it exhibits on the application of galvanism.

The par vagum is evidently a nerve of respiratory motion; the branch of the fifth and that of the sixth, which form the whole, or constitute a part of the sympathetic, transmit motion or sensibility; and the whole of the spinal nerves are proved to 
possess the same powers; and as these are combined in every thoracic or abdominal plexus or ganglion, it is reasonable to suppose, that the connexion of the various fibres cannot in the least change their individual properties : before they contribute these properties, they exhibit sensibility and motion; and if they are conjoined, the centres which they form will be sources of sensibility and motion.

CCXXXVI. If then the whole of the ganglions and plexuses be centres or combinations, participating in the endowments of nerves whose functions have been ascertained, it follows that the viscera of the thorax and abdomen, the extremities and surface of the body, are supplied with nervous energy composed of one or both of these qualities. If this view be taken, we leave no nerves to perform secretion or excretion, except it be allowed to the physiologist that the nerve which performs motion in one part of the body is a nerve indispensable for secretion in another. This idea has been embraced by Wilson Philip and others, who believe that the formation of the gastric juice depends on the eighth pair of nerves.

CCXXXVII. When I speak of sensibility and motion, I do not, by any means, confine those terms to the limits of their ordinary acceptation. I look upon every organ as possessing sensibility and motion corresponding to its office. The contraction of the heart, iris, or sphincter 
ani,-the peristaltic action of the intestines and stomach,-the elevation of the ribs, - or the inordinate action of any of the muscles belonging to respiration,-in fine, every motion or contraction of muscular fibre, I regard as depending on nervous fibrilla originating from the nerves of motion, modified by the organization of the part or organ.

CCXXXVIII. Every organ of the system has its proper stimulus : the eye has light, the heart blood, the stomach has food and its peculiar secretion, and the whole of the abdominal viscera their various agents; but the susceptibility of an organ to its stimulus will be proportionate to the fasciculi derived from the nerves of sensation, and the energy with which it acts will be according toth e number or size of fasciculi belonging to the nerves of motion, and the adaptation of the part to promote the intended effect. If we examine the structure of the iris, we shall observe its radiated and circular fibres, which are, by the best authorities, considered muscular; if then light be transmitted to the retina, the iris contracts in virtue of its sensibility to impressions, and the power of motion communicated to its radiated and circular fibres by its nerves of motion. If we examine the disposition of the muscular fibres of the sphincter ani, we observe that these are calculated to produce the necessary effect; or if we look to the stomach and intestines, we have circular fibres 
which are continually in action for the purpose of propelling or otherwise acting on the contents ; in the intercostal muscles, and others appropriated to respiration, we have longitudinal fibres,-and it is by structural modifications of this description that the various kinds of contraction are produced. Sensibility and motion may be considered as fundamental properties bestowed on all organs, differing only in extent, while the character of the contraction is regulated by its muscular conformation and the nature of its stimulus. These properties in the state of health are uniform in their influence, but in disease they are much disordered. In Enteritis the natural sensibility of the bowels is heightened, and transmits to the sensorium painful impressions, and, at the same time, the anti-peristaltic action occasionally occurs, showing that the nerves of motion are equally deranged. These or similar observations may be applied to every part of the body.

CCXXXIX. I have alluded to the stimuli of several organs : it is also necessary to remark that these generally place the function beyond our control. The contractions of the heart, the assimilation of food, and the different secretions, are illustrations; and yet we observe that the will can influence certain functions which are partly voluntary and partly involuntary. In what way the will operates is unknown to us, nor does the 
investigation particularly concern the physiologist-it is sufficient for him to observe that different acts of the system are accomplished by the simple volition of the mind.

CCXL. Whether the sympathetic nerve arise from the brain, or only be connected with it, or whether the system which it forms is to be regarded as independent of the cerebrum and spinal cord, or as constituted to combine and communicate the same influence in the more perfect animals, are subjects which will continue to perplex as long as anatomy be appealed to as the only guide.

If the ganglionic system in the lowest orders of animated nature be supposed to preside over the functions of secretion, absorption, and nutrition, as there is no other system of nerves present, it may be justly asked whence those powers are derived which bestow sensibility and motion? MECKEL and WEBER remark, that the sympathetic nerve and par vagum are developed in the inverse ratio of each other. In the lower gradations of animal life, the sympathetic nerve, or ganglionic system, is the only nervous structure which is present for sensation and motion; and, as we ascend in the scale of being, we perceive the additional contribution of the par vagum to supply the multiplied wants of the animal. It is, therefore, probable that these two nerves possess endowments precisely of the same 
kind so far as regards motion, but that the sympathetic, besides this common property, has the power of conferring sensibility also.

It has been proved, that the sympathetic nerve is capable of being excited by galvanism. Schmuck, Fowler, Pfaff, Ludwig, Creve, and WEBster, have excited the action of the heart, both in warm and cold blooded animals. Humbold has also produced contractions in some of the inferior orders of animals, as the limax ater, ostrea edulis, helix pomatia, helix nemoralis, sepia officinalis, by the application of galvanism. It has also been shown, that the diaphragm can be sensibly excited by the same means.*

CCXLI. All these arguments and direct experiments tend to persuade or demonstrate, that the function of the sympathetic nerve is the same as that which belongs to the cerebral and spinal nerves, viz. to distribute sensibility and motion to almost every part of the thorax and abdomen in the class mammalia, and in the inferior orders to diffuse these properties throughout the whole system.

CCXLII. It was imagined by Sømmering and Behrends, that the numerous nervous fibrillae sent by the sympathetic nerve to the heart are not blended with the muscular fibres, but simply accompanied the vessels of circulation. It has since been shown, that these are as intimately connected with them as the voluntary

* Lobstein, p. 174. 
nerves are with any of the muscles of the body. When galvanism is applied to the sympathetic nerve, which principally supplies this organ, the contractions are increased, because it stimulates the various nervous ramifications in the same manner as the vital fluid; and from what I have previously stated, it is obvious that the contractions of the heart are modified, in almost every possible way, by the qualities of the blood.

CCXLIII. Many experiments have been performed for the purpose of ascertaining how far the capillary circulation is influenced by the destruction of the brain or spinal cord, as if it were possible by such means to know the precise relations existing between the capillary circulation and the nervous mass. If we consider that the removal of the cerebrum must derange the general circulation according to the quantity of blood which such an operation takes from the system, not simply from the blood which is lost in the excision of the vessels, but from what is proper to the organ, or, if we reflect that great disorders in the sanguiferous system will otherwise be induced, how is it possible to draw any positive conclusions from such effects and causes, when a variety of others intervene of which no estimate or conception is formed? Though we allow that the capillary circulation is independent of the brain and spinal cord, and this opinion can scarcely be disputed, yet we cannot for a 
moment suppose that it is equally independent of the general circulation. It will be subject to two great modifications: The quantity, and condi. tion of the vital fluid transmitted to its vessels. If the properties of the blood be highly stimulating. the same will immediately influence the circu. lating powers of the capillaries; if the quantity which is sent is either greater or less than usual, these will be sensibly affected; and if we employ chemical or mechanical means which produce one or both of these circumstances, the results cannot be regarded as affording any indications of nervous influence.

CCXIIV. The sympathetic nerve is almost universally supposed by anatomists and physiologists to be indispensable to the offices of secretion, nutrition, and circulation, and is also imagined to be the source of a multitude of diseases. LoBstern, in enumerating the Pathemata istius nervi essentialia, includes in the list Hypochondriasis, Morbus Hystericus, Melancholia, Mania, et Febris Intermittens. I shall not, at the present, attempt to show the fallacy of such opinions : the refutation of the greater number will be found in the different views developed in this work; and the more minute investigation of the subject will be deferred until we treat fully of the class of nervous diseases.

CCXLV. The principal arguments hitherto advanced to prove that organic functions are performed by means of the sympathetic nerve and 
par vagum, may be reduced to the following propositions :

1. If the par vagum be cut, the secretion of gastric juice is arrested.

2. Galvanism has been shown to supply the want of nervous energy, by accelerating digestion, after the destruction of the eighth pair of nerves.

3. The nerves accompany the arteries and muscular fibres in their most minute ramifications.

4. Many nervous fibrillae are distributed on those organs, which are destined either for the circulation of blood or the assimilation of food.

5. The development of the nervous system increases with the augmentation of additional organs or their more complete organization.

6. The secretion of gastric juice is materially retarded by the exhibition of narcotics, whieh are supposed to act on the nerves.

7. Mental influence occasionally disorders the thorax and abdominal functions.

In Chapter III. I examined the opinions of Wilson Philip in regard to the effects arising from the destruction of the par vagum; and therefore it is not necessary to repeat the same observations to show that the first proposition is incorrect. The experiments at the end of this chapter will satisfactorily prove that the secretion of gastric juice is stopped, not on account of nervous energy being diverted from its course to the stomach, but on account of the great disturb- 
ance which such an operation occasions in the qualities and distribution of the blood. It has also been shewn in what manner galvanism maintains the function of digestion. It operates as a stimulus, and tends to remove congestion, whether in the lungs or stomach, and thereby allows the former to oxygenate properly the fluid which it receives; and this, possessing its usual properties, is transmitted to the viscera of the abdomen; the consequence of which is, that the organic functions are supplied with blood calculated to support their action. The truth of this view is found in perusing the cases which WrusoN Philip himself relates. The immediate effect of galvanism was to relieve the oppressive breath. ing; and, in those instances in which this was employed, the lungs were comparatively healthy, whereas, in those in which it had not been used, the lungs presented "dark red patches," like the lungs of an animal that had "lain dead" for many hours.

CCXLVI. In proposition 3d, we may observe, that sensation and motion, variously modified, belong to every part of the system. The universal dissemination of these qualities of matter makes an individual sensible to external agents, and gives him the faculty of removing from, or approaching to such, according as these are painful or agreeable; and it also binds in harmony the different organs of the body, facilitating mo, 
tion where it is required, and transmiting sensation when it is necessary.

CCXLVII. The accurate plates of SCARPa point out the great number of nerves which the heart receives from the sympathetic and par vagum; and if the distribution of these to an organ is a powerful argument in support of their influence in secretion, it may be asked, what does the heart secrete? But if they be allowed to give motion and sensibility, it is evident that the function of the organ satisfactorily accounts for the multitude of nervous fibrillae. Its constant contractions are effected by its nerves of motion, which endow its muscular fibres with a property called irritability, and, unless it possessed this extent of nervous energy, the blood would not be able to maintain its regular action. Secretion and nutrition are accomplished in the vegetable without the assistance of nerves; and this fact, from the similarity of the functions in both instances, naturally leads the mind to conclude that these are the result of laws not directly depending on the nervous system.

CCXLVIII. As we rise in the scale of being, additional and more perfect organs are developed, and the nervous system is also proportionately augmented to meet the demands of these superadded powers.

CCXLIX. The manner in which narcotics influence the generation of heat, and the secre- 
tion of gastric juice, will be considered in the next chapter.

CCL. The different conditions of the mind have been known, from the earliest observations, to excite or depress the digestive and assimilating organs; but as this subject is fully treated of in a subsequent chapter, it is unnecessary, at present, to make any remarks upon it.

CCLI. It has been observed by every one who has paid particular attention to the division of the par vagum, that great difficulty of breathing immediately follows this operation, if performed on both sides, or if the nerves be tied; and although this effect was so obvious, the cause was not accurately ascertained till LEGALLoIs proved it by direct experiment. It was demonstrated that, when the par vagum was divided, the superior and inferior laryngeal branches, distributed to the muscles of the larynx, no longer enabled them, as in health, to open and close the glottis,-the consequence of which is, that atmospheric air cannot pass in its usual quantity into the chest to produce the ordinary changes of the blood. Although the correctness of this explanation has never been questioned, yet a variety of opinions have been entertained in regard to the cause of death.* Dupurtren supposed that the air entered the lungs, but did not properly oxygen-

* Vide pages 180-1, 200, 2C2, 224, 235,-LEgALLors. 
ate the blood: this was remarked to be of a dark colour, and the temperature of the body was also found diminished. "Il pensa donc, $\mathbf{1}^{\circ}$, que tous les animaux auxquels on a coupé les deux nerfs pneumo-gastriques meurent d'asphyxie; $2^{\circ}$, qu'ils en meurent, parceque l'air atmospherique, quoique continuant de pénetrer librement dans les poumons et d'y arriver en contact avec le sang, ne peut plus se combiner avec ce fluide, cette combinaison ne pouvant se faire que sous l'influence du principe vital et par l'intermediaire dés nerfs."*

Blainville imagined, with Halier and many other physiologists, that the death of the animal was to be referred to disorder of the digestive powers, and various changes of the contents of the stomach; others believed that some great alteration was communicated to the heart, by which means the circulatory system was destroyed or disturbed. LEgallois himself conceived the death of the animal to be occasioned by the three following causes: " $1^{\circ}$, Par la diminution de l'ouverture de la glotte; 20, Par l'engorgement sanguin des poumons; $3^{\circ}$, Par l'epanchement d'un fluide sereux dans les bronches." † LEGALLoIs, in speaking of the effects arising from the division of the eighth pair, says, "la section des deux nerfs affecte à la foìs le larynx, le coeur, le

* Legallois, p. 178.

† Ibid.p. 235. 
canal alimentaire et les poumons."* $\mathrm{He}$ explains the affection of the larynx, but the other consequences are difficulties which he does not solve.

CCLII. From the great and general disorders of the viscera, of the thorax and abdomen, it is not easy, unless we seize the first and principal agent in the chain of causes, to account for the extraordinary effects produced. Impeded digestion, congested lungs, and a vascular state of the heart, have been described by the greater number of those who have prosecuted the present subject; and the following experiments, from their conclusive character, will show why such simultaneous results occur, and what is the nature of the relations existing between the different organs.

The following experiments are to prove these propositions :

1. That the function of the par vagum, and its various branches, is to regulate the ingress and egress of atmospheric air in the act of respiration.

2. That nervous influence is not necessary for the secretion of gastric juice.

3. That the first cause in the disturbance of the organic functions is to be referred exclusively to the injury done to the laryngeal nerves, by which means the usual quantity of air does not enter the lungs.

4. That the death of the animal arises from the three causes stated by Legaliois.

$$
\text { * Legallois, p. } 231 .
$$




\section{EXPERIMENT I.}

The influence of the par vagum on the function of digestion.-The three rabbits in this experiment were adults, and about the same size. They were fed at half past six o'clock, A. M. with cabbage leaves; they had previously fasted sixteen hours. This rabbit had the par vagum divided on one side only, at eight o'clock. Temperature before experiment $1021^{\circ}$.

Time. Temperature.

10 o'clock 4. M. $100 \frac{1}{2}$ 。

12, noon,

2, P. M.

6, Р. м.
$101 \frac{\pi}{4}$

102

103
Observations.

Apparently cheerful.

ditto.

ditto.

ditto.

At six o'clock, P. M. this rabbit was killed.

Dissection.-On opening the chest the lungs collapsed, but were slightly infiltrated; the heart appeared in the natural state ; nothing worthy of particular remark in the thorax. The stomach, of a smaller size than in the two others, was filled with a homogeneous mass, in which the well-marked characters of vegetable food were absent.The contents of the large intestines were well concocted. The weight of the lungs and heart of this rabbit was three drachms and a half.

In the following, the par vagum was divided on both sides. Temperature of the rabbit, before experiment, $105^{\circ}$. The operation was performed a few minutes after the preceding.

Time. Temperature.

Observations.

10 o'clock A. M. $\quad 98^{\circ}$ Respiration very laborious.
12, noon, $97 \frac{1}{4}$
2,
P. $\mathbf{M}$.
96
P. M. 93
ditto.
ditto.
ditto.

At six o'clock, P. M. it was killed. 
Dissection.-The heart and lungs of this rabbit were much larger than usual. The lungs were very much engorged, presenting several large patches of a dark hepatic colour. The heart was very turgid, and the vena cava of the thorax and abdomen very much distended with dark venous blood; the stomach of more ample dimensions than in the preceding; the contents occupying the larger arch of the stomach had undergone no perceptible change; the rest were better, but still coarsely, digested, exhibiting strong traces of vegetable fibre. The large intestines contained a mass only somewhat better concocted than that in the stomach of the former. The lungs and heart of this rabbit reeighed seven drachms.

In the following rabbit, three quarters of an inch of the par vagum, on each side, were cut out. This operation was performed immediately after the preceding. Temperature of the rabbit before experiment $104 \frac{1}{2}$.

At ten o'clock, A. M. the respiration was extremely laborious; the temperature $100 \frac{1}{2}$. From this hour the respiration increased in difficulty till noon, at which period, in extreme agonies, the rabbit jumped against the wall, and expired. At this moment I separated the trachea, for the purpose of transmitting air into the lungs, thinking that it might revive the animal-but it did not. On opening the trachea a quantity of frothy matter flowed out, and continued to do so for the space of a quarter of an hour. The quantity was sufficient to fill a table-spoon.

Dissection.-The thoracic viscera of this rabbit exhibited striking peculiarities. The lungs dense, no longer crepitant, of a deep Modena hue, with circumscribed patches of a much darker colour, entirely filled the cavity of the thorax. The air cells on pressure effused a frothy matter. The vena cava of the thorax and abdomen was very much distended with black blood. The stomach 
was much larger than in the two preceding. The contents were of a less firm consistence, but did not display the same unaltered appearance as in the large arch of the stomach of the former rabbit, being almost as much digested as the best concocted portion in the stomach of that animal. The large intestines were in the same condition as those of the preceding rabbit. The lungs and heart weighed thirteen drachms and a half.*

\section{EXPERIMENT II.}

From the sudden death of the last rabbit, and from the whole of the thoracic symptoms, I proposed, in this experiment, to obviate the severity of the consequences by dividing the trachea, and adapting to it a large brass tube, to allow the atmospheric air to enter the lungs freely. The three rabbits in this experiment were full grown, and appeared equally healthy. After fasting for twenty-three hours they were allowed to eat plentifully of cabbage leaves five hours before the operation, immediately before which a small quantity was again given.

Temperature of this rabbit $103^{\circ}$. The trachea was divided, and the tube attached as described. About half an inch of the par vagum on both sides was cut out. The experiment commenced at one o'clock $\mathbf{P}$. $\mathbf{M}$.

At three o'clock the temperature was $104^{\circ}$. The rabbit breathed as easily as in the state of health. Tantillum quidem hic secunda post sectionem factam hora pati videbatur, ut femellam etiam ascenderet, atque cum ea coire

* To ascertain the weight of the lungs and heart of a rabbit in health, the viscera were taken from two adult rabbits that had been instantaneously killed. In the one they were found to weigh two drachms and a half, in the other three drachms and one scruple. 
haud segniter conaretur. At half past four it seized with avidity a cabbage leaf, but seemed very much oppressed in the attempt to swallow. There was excited for some minutes a state of respiration not much unlike sneezing, and it seemed, from the sound emitted, as if the tube was not fully pervious. After a little time the rabbit resumed the ordinary mode of breathing. At five o'clock the temperature was $102{ }^{\circ}$. At seven $o^{\prime}$ clock the temperature was $100^{\circ}$. The natural character of the respiration was unchanged, but the animal appeared to be in considerable pain. Its eyes were almost closed, the head raised up as if to allow the air to enter the tube; and its otherwise motionless attitude, and occasional catches in its breathing, indicated great uneasiness and suffering. Matter of a sanious and frothy appearance exuded from the wound in the neck. At nine o'clock the temperature was $99 \frac{1}{2}$; the rabbit was very dull and inanimate; respiration as before, the occasional catches more frequent. At eleven o'clock the temperature was $99 \frac{1}{4}$; the rabbit still dull and inanimate, refusing to eat. At one o'clock A. M. the temperature was $103^{\circ}$. The rabbit appeared more cheerful, and was inclined to eat. It was left in this state till seven o'clock that morning, at which time it seemed the same in every external respect. An hour after this its temperature was taken, and found to be $101^{\circ}$ : the rabbit was then killed.

Dissection.-The lungs did not collapse so perfectly as ordinary, but were still crepitant, and of the natural appearance. The stomach, larger than usual, was filled with a mass intermediate in every respect to the contents of the two following; the vegetable fibre and smell were still evident. The contents of the large intestines differed little from the natural state. The osophagus was entirely filled with imperfectly digested vegetable; and this circumstance 
accounts for the oppression which the animal experienced in the attempt to swallow what it had chewed. This organ did not seem to have the power to propel the food received, and consequently its great distention would more or less compress the trachea, causing the symptoms I have described on giving it food at half-past four. Great inflammation and congestion were observed round the wound; and the parietes of the thorax.

In the following rabbit the par vagum was divided on both sides. Temperature $104^{\circ}$. The trachea was not divided.

At three o'clock the temperature was $100 \frac{3}{4} \circ$. At halfpast four o'clock the rabbit received cabbage leaves, which it voraciously seized ; but, after having taken a small quantity, vomiting commenced, and continued, with slight intermissions, till a quarter to five, at which time the animal died. The respiration had very much increased in difficulty for two hours previous to its death.

Dissection.-The lungs did not collapse on dividing the parietes of the chest, but they were crepitant in almost every part : they were almost as large again as the healthy lungs. The superior surface was highly red, presenting many small black points and slight discolorations; the inferior was marked with black patches, The greater part of two lobes, in this situation, was in appearance like the liver.

The condition of the heart was natural ; the veins of the chest and abdomen were large, and much distended with black blood; the stomach much larger than usual; the principal part of the food appeared to have undergone no change whatever; the portion which was most acted upon was still unaltered in its vegetable character, but was rather minutely divided. The contents of the large intes- 
tines were coarser and less perfectly concocted than ordinary.

In the following rabbit, the par vagum was divided on one side only. T'emperature $104^{\circ}$.

\begin{tabular}{|c|c|c|}
\hline Time. & Temperature. & Observations. \\
\hline 3 o'clock & $105^{\circ}$ & Appearance healthy. \\
\hline 5 & $104 \frac{1}{2}$ & ditto. \\
\hline 7 & $104 \frac{1}{4}$ & ditto. \\
\hline 9 , & $104^{4}$ & ditto. \\
\hline 11, & 103 & ditto. \\
\hline 1, A. M & 103 & ditto. \\
\hline 8, ditto, & 104 & ditto. \\
\hline
\end{tabular}

At eight o'clock in the morning the rabbit was killed.

Dissection.-The lungs collapsed perfectly, but seemed somewhat of a darker colour. The stomach, of a moderate size, was filled with a mass that had lost the coarse and chopped appearance of undigested vegetable, yet presented the vegetable smell, and here and there the vegetable texture. The whole contents, at a superficial glance, appeared homogeneous. The large intestines exhibited the conditions of health.

\section{EXPERIMENT III.}

The three rabbits employed in this experiment were full grown, healthy, and equally vigorous. They had fasted twenty hours before they were fed with dandelion at eight o'clock in the morning. The operations were performed at half-past eight. In the following rabbit the trachea was cut, and a tube attached to it, as in the preceding experiment. Its temperature was $103^{\circ}$. The nerves were left intact.*

At two o'clock the temperature was $104 \frac{1}{4}^{\circ}$; respiration regular and easy. It seized with avidity the food which was offered. From this time till ten o'clock at night there

* The trachea only in this and some other experiments was cut, in order that we might estimate the influence of such a serious operation on the digestive powers. 
was little particular to observe ; its appetite appeared as keen as ever, but its respiration was occasionally laborious. Its temperature was $104^{\circ}$. At half-past seven the following morning this rabbit was found dead, but was still warm.

Dissection.-The lungs were somewhat engorged, and were of a bright Modena colour ; they collapsed but slightly on opening the chest. The stomach was large, and its contents were of two very opposite descriptions ;-that occupying the cardiac portion was vegetable, slightly divided, but not otherwise altered in appearance; that of the pyloric was well comminuted, and apparently homogeneous. The contents of the large intestines were moderately well concocted.

The trachea in the following rabbit was divided, and the par vagum on one side only. Temperature of rabbit $105^{\circ}$.

At two o'clock the temperature was $104^{\circ}$; respiration natural; great avidity for food. At this time the rabbit alios ascendere sapius conabatur, et, quanquam' hebetior erat, nihilominus blandiendo variis modis salacitatis speciem reprosentabat. This is a phenomenon I cannot in the least account for. A gentleman was with me when I observed the same in Experiment II. I am persuaded that it does not arise from the vigour of the animal, nor from the natural impulse of the feeling; for, in the present instance, the first selected was a very fine male rabbit, and it very frequently approached, without any discrimination of sex or age, the individuals among which it was placed. It has never occurred when the trachea only was cut, and, since it has happened in these two cases in which the par vagum was divided, it would seem to depend on some derangement of the cerebellic system. But, as it is my in- 
tention to state facts and not hypotheses, I shall leave the reader to form his own judgment on this subject. At ten o'clock at night the rabbit appeared as usual, and was disposed to eat. At half-past seven o'clock next morning its breathing was laborious, and it refused to eat. 'The temperature was $98^{\circ}$. At this time it was killed.

Dissection.-On opening the chest the lungs collapsed, and were of a perfectly healthy appearance, and crepitant throughout. The contents of the stomach were homogeneous, and of a very soft consistence. In different parts of the stomach undigested vegetable was evident; but the whole mass was as well digested as the pyloric portion of the preceding, but scarcely equal to that of the following. The contents of the large intestines were fluid; but from this circumstance no inference can be drawn, as the intestines were in a state of high inflammation, and were agglutinated in several situations.

The trachea of this rabbit was divided, and the tube attached as in the preceding; about half an inch of the par vagum, on each side, was cut out. Temperature of the rabbit $104^{\circ}$. At two o'clock the rabbit was breathing as in the state of health. At ten o'clock the respiration had become slightly laborious and hurried. Its temperature was $98^{\frac{1}{2}}$. It died in about seven minutes after I had ascertained its temperature. The cause of its sudden death I could not account for. It did not appear particularly oppressed before the introduction of the thermometer into the rectum. The chest was immediately opened, and presented the following appearances.

Dissection. - The lungs collapsed on opening the chest; they were somewhat of a darker colour than natural, but did not present any traces of inflammation, nor did we observe any dark patches; they were of the usual size, and 
crepitant throughout. The liver was very large, and diminished the capacity of the thorax. The stomach was of the usual dimension, and contained a small quantity of air. The contents appeared homogeneous; but when examined, there were here and there the remains of vegetable fibre; yet the whole mass was minutely divided, and exhibited a more advanced progress towards perfect digestion than that of the two preceding. The contents of the large intestines seemed to be well concocted; the small intestines were distended with air to a great extent.

\section{EXPERIMENT IV.}

In this rabbit the trachea only was divided. Temperature $105^{\circ}$. At five o'clock the respiration was natural, and the rabbit was inclined to eat. At seven it appeared perfectly well, and had great avidity for food ; in ten minutes after it jumped out of the box in which it was kept, ran once half round a large room, and in a few seconds expired in struggles. The frequency and suddenness of this circumstance was mortifying, as it interfered with the train of experiments, but more so from my not being able to account for it. It is so natural for the mind to attribute extraordinary effects to extraordinary causes, that I did not imagine, till the present instance occurred, that the phenomeyon might arise from the stoppage of the tube, which I found to be the case; and the knowledge of this fact enabled me to save the life of the following rabbit; and it also accounts for the previous accidents of the same kind. The tube is so liable to become partly or completely impervious, that the experiment is rendered delicate, and sometimes unsatisfactory.

Dissection.-The lungs were somewhat congested, of a deeper colour than natural, and, although perfectly crepitant, did not collapse so completely as in the former instance. The stomach, of its usual size, was filled with 
imperfectly digested food, which exhibited, especially towards the cardiac extremity, many pieces of unaltered vegetables; towards the pyloric, though somewhat better digested, it was by no means so much so as in the latter rabbit. The contents of the large intestines appeared perfectly concocted.

The trachea of the following rabbit was not divided till the difficulty of breathing was obvious. About half an inch of the par vagum on both sides was cut out. Temperature 1023ㅇ. At five o'clock the respiration differed slightly from the state of health: the rabbit was disposed to eat. At seven o'clock the respiration easy. At eleven it was difficult. At one, A. M. the rabbit ran across the room, and struggled, as the preceding had done, at seven. Perceiving this, and believing it to originate from the same cause, I passed a probe down the tube attached to the trachea, and had the satisfaction of seeing the animal revive and breathe with almost the ease and regularity of health. At eight o'clock in the morning the animal seemed quite worn out; its breathing was rather hurried and weak, but not particularly laborious. The rabbit reclined partly on its side; and, when raised up, a small quantity of mucus was observed to ascend in the brass tube, accompanied by a sound similar to what is heard on applying the ear to the chest of a phthisical patient in whose lungs large cavities exist. At this time it was killed. The temperature was taken immediately after death, and found to be $105^{\circ}$.

Dissection.-On opening the thorax the lungs collapsed, and were of a healthy appearance in every part except the posterior lobe on the left side, which was of a much darker colour than the rest; they were throughout crepitant. The stomach, of a small size, was filled with a perfectly homogeneous matter of a soft consistence, and which had 
eniirely lost the characters of vegetable food. The contents of the larger intestines were more completely concocted than any that $I$ have observed in the whole series of these experiments. The neck of the animal was dissected after having examined the thoracic and abdominal cavities, and the divided ends of the par vagum on each side were readily discovered.

The two rabbits in this experiment were seven weeks old. They had partaken of a small quantity of dandelion at one o'clock, P. M.; the operation was commenced an hour afterwards.

CCLIII. The preceding experiments are conclusive and important in their character. We have now no longer any uncertainty concerning the principal-function of the eighth pair of nerves; nor are we perplexed in endeavouring to explain the consequences which follow their division. In Chapter III. I endeavoured to show the fallacies in the reasoning of WILson PHILIP, and stated that the beneficial influence of galvanism was not to be attributed to any similarity or identity with the nervous fluid, but to its general stimulating properties ; and I have by the present experiments shown, if a tube be attached to the divided trachea, although the nerves be cut, that digestion goes on as rell as when the trachea alone is divided. From such facts, which it is unnecessary to multiply, we prove, that whatever disorders the proper oxygenation of the blood, or disturbs its distribution, deranges the function of digestion; and, still fur- 
ther, that whatever maintains or re-establishes the regular action of the thoracic viscera, maintains or re-establishes the function of digestion, not because the nerves are affected by such changes, but because the secretory organs depend on these conditions of the blood.

CCLIV. If from a similarity of effects we are authorised to infer a similarity of causes, we may state that the admission of air into the lungs by means of a brass tube is identical with galvanism and the nervous fluid; but those who support the opinion that the two latter are the same or similar, will be unwilling to allow this conclusion, because we know that atmospheric air is not galvanism, nor does it possess any properties like those ascribed to the nervous fluid.

CCLV. In rabbit third, Experiment I. it is stated that the lungs and heart were about four times as heavy as those belonging to the first; and these results are in harmony with the symptoms observed during life. In the one, respiration was performed with facility; in the other, the breathing became difficult immediately after the operation, and the urgency continued to increase till death, which happened in four hours after the excision of a part of the nerves. The additional density and increased size of the lungs and heart, and the great distention of the thoracic and abdominal veins, explain the cause of death and the phenomena remarked previous to this event. 
CCLVI. When the par vagum was divided on both sides of the neck,* the aperture of the glottis was much diminished from the paralysis of its muscles; and this accounts for the difficulty of breathing which almost directly succeeds the operation. Although the ingress of air is, in a great measure, prevented, yet the blood returns for some time to the heart and lungs as in health; but when it arrives there it is impeded in its circulation, nor does it undergo the necessary changes; the consequence of which is, that it gradually accumulates, and ultimately renders inefficient for the continuance of life, the small quantity of air allowed to pass through the lessened aperture of the glottis. If this rabbit had lived a little longer, we should have observed a great diminution of temperature. In the second of the same experiment, the thoracic disorders were intermediate in degree between the first and third, and yet its temperature was reduced $12^{\circ}$; and this effect is in harmony with the first general principle, viz. that animal heat is not in the direct ratio of the quantity of oxygen inhaled, but in the inverse ratio of the quantity of blood exposed to this principle.

\section{EXPERIMENT ' $V$.}

The two rabbits employed in this experiment were between two and three months old, apparently healthy.

* Vide. Appendix. 
They had fasted fifteen hours, when they were fed at seven o'clock, A. M. The experiment commeneed at eight o'clock. The par vagum on each side was tied in the following rabbit. Temperature before experiment $103^{\circ}$. The animal cried immediately on the nerves being tied, and respiration was difficult from the commencement. Vomiting and great difficulty of breathing were very oppressive directly after the operation, and the former continued so for a quarter of an hour; the latter increased in urgency throughout the experiment. At eleven o'clock the temperature was $99 \frac{\mathrm{x}}{2}$; respiration very difficult. The animal displayed an avidity for food, but had not the power of eating. At one o'clock, respiration short and laborious. I attempted to take the temperature, but, from the difficulty of breathing, it was impossible, with safety to the life of the animal. In half an hour after this time it died. Its temperature was found to be $93{ }_{4}^{\circ}$, taken when insensible, but not dead.

Dissection.-The lungs did not collapse on opening the thorax, were scarcely crepitant in any part, and were similar, in almost every respect, with the exception of here and there a few light points, to a mass of coagulated venous blood. The veins of the thorax and abdomen were distended with black blood. The stomach, of a moderate size, was filled with food, of which that part contained in the large extremity appeared not in the least acted upon, while that in the small was rather more digested. The contents of the large intestines were natural. The thoracic viscera weighed 4 drachms and a-half.

The par vagum on each side was tied in the following rabbit. Temperature $103^{\circ}$. Difficulty of breathing commenced immediately, but was not so urgent as in the preceding animal. At eleven o'clock respiration laborious; temperature 
was not taken, from the struggles which the attempt occasioned. A few minutes after the death of the foregoing rabbit it was on the point of expiring, which induced me to kill it. Temperature $91 \frac{3}{4}^{\circ}$, taken when insensible but not dead.

Dissection.-The lungs were precisely in the same state as in the preceding rabbit; and also the veins in the thoracic and abdominal cavities. The stomach was filled with food, of which that portion contained in the large extremity appeared to be vegetable quite unchanged; that part in contact with the mucous surface was more minutely divided than the rest. The contents of the large intestines were natural. The livers in both these rabbits were tuberculated. The thoracic viscera weighed four drachms and a-half.

CCLV1I. It had been remarked by HALLER and Bichat that respiration was extremely difficult in cases in which the par vagum was divided; but we were not aware of the cause of this till the decisive experiments of LEgar.LoIs, and those which I have performed, prove, that dyspnœa,-impeded or obstructed digestion,enlargement of the heart, - congestion and disease of the lungs, - distended state of the internal veins, and diminution of temperature,-are readily prevented by dividing the trachea, and attaching a tube to it at the time that the nerves are cut.

CCLVIII. For the purpose of observing with more accuracy the results of the different experiments, the rabbits were placed under my own immediate eye, in a room to which they were 
strangers. On this account they generally became dull and inanimate, and I found this to be the case with others which had undergone no operation. When removed to their old abode they ran about with their accustomed cheerfulness. Mental depression influences the digestive powers of a rabbit as well as the more complex functions of an intellectual being. I mention this circumstance, as I am convinced that it ought not to be neglected in experiments which are instituted for the purpose of estimating the value of any single organic law in regard to the function of digestion or any of the secretions of the system. 


\section{CHAP. XI.}

\section{The Influence of Narcotics on the Generation of Animal Heat and the Digestive Powers.}

CCLIX. In undertaking the present subject, it is not my intention to investigate the individual properties of the different narcotics, the consideration of which would occupy considerable time, and would necessarily exclude from this book other topics more directly bearing on the general principles expounded. Opium; whether regarded as a medicine or poison, is more important and interesting than any included in the general class of these substances, and, on this account, I shall select it for careful but general examination.

CCLX. From the variety of effects consesequent on the exhibition of opium, much diversity of opinion exists concerning its true action. Some are disposed to consider it as a sedative, others as a stimulant. Those who support the former quality adduce the well known symptoms of somnolence, prostration of muscular. power, insensibility to external and internal agents, and the occasionally weak or slow contractions of the heart. But, on the other hand, 
those who assert that it is a direct stimulant, lay great stress, and with justice, on the more frequent occurrence of excited action, such as the forcible contractions of the heart, strong and frequent pulsations of the arteries, and increased temperature, displayed occasionally in the flow of perspiration or heat of surface.

Some individuals entertain opinions less exclusive in their character, and regard opium as possessing both these properties. The stimulant effects, they say, arise from the smallness of the dose, and the sedative from an excessive quantity. This explanation is consistent with certain facts, but is far from being satisfactory, when applied to the different phenomena of the system, originating from the influence of opium.

CCLXI. Our ideas concerning the characters which constitute a sedative or stimulant, are neither exact nor precise, and, until we have more definite conceptions of these characters, we shall continue to dispute about the action of opium. If a sedative be defined to be a power that depresses the mental and corporeal faculties, evinced by the incapability or insensibility of the mind, and a diminution of the contractions of the heart; and if a stimulant be defined to be an agent which excites the intellectual and physical endowments of the system, as indicated by an unusual flow of animal spirits, and increase of muscular force, we are fully prepared to examine the merits of those 
theories proposed to solve the difficulties of the present subject.

CCLXII. If the above definitions were considered correct, they would be fatal to the views of those who assert that opium is a sedative or stimulant,-for, in the greater number of instances in which the former property is exhibited, the action of the heart is augmented; and when the latter is supposed to be present, from the great vigour exhibited by the heart and arterial system, the faculties of the mind are depressed or incapacitated from performing their ordinary functions. It is, therefore, manifest that contradictions and inconsistencies exist in the various opinions brought forward.

CCLXIII. Fxperiments and observations will probably enable me to explain the action of narcotics on the animal economy, or, at least, to present views that apply more generally to the effects which succeed their exhibition. The different organs of the body are variously constituted, and the functions which they have to discharge possess neither the same delicacy nor ob. ject. We have organs whose office it is to contract, secrete, or to develope the intellectual powers; and as these are in every respect so differently formed, can we for a moment imagine, although parts composing one great system, that they will be equally influenced by the same general agent? It is as reasonable to suppose that 
the eye will be affected by what is painful to the ear, as that these organs will suffer to the same extent by the general operation of opium or any other agent.

CCLXIV. The heart and the brain reciprocally and almost immediately act on each other. If the former be excited, it almost directly communicates to the brain a portion of the energy which it displays; and if the latter be deranged or diseased by congestion or external injuries, it disorders the action of the heart, by perverting the regularity of the respiratory functions. By rendering the respirations less frequent or complete, the qualities of the blood are deteriorated; and as the motion of the heart depends on these, it will necessarily be implicated in those conditions of the cerebrum by which the respiration is deranged.

CCLXV. When a small quantity of opium has been taken, it is absorbed, and carried by the circulation to every part of the body. The heart is increased in its contractions, because its nerves are directly stimulated; and the faculties of the mind are also excited, exhibiting a state of simple exhilaration, or delightful reverie, from the operation of the same cause upon the brain. If a still larger quantity be taken, the mental powers are still more disturbed, showing the sedative effects of opium, such as insensibility to impressions, or stupor, accompanied by stertorous or interrupted respiration; but while the sangui- 
neous fluid occasions these consequences in the delicate and nervous structure of the brain, it still remains for some time a stimulus to the heart, because this is differently organized. But it not unusually happens that the dose is sufficiently great to enfeeble the motions of this organ, at the same time that it disorders the functions of the brain.

CCLXVI. I have observed in many experiments performed on rabbits, that a small quantity of opium, in the greater number of instances, augments the action of the heart for a short period, while a greater proportion almost immediately diminishes the force and frequency of its contractions. If such circumstances occur in an animal made insensible, it is reasonable to suppose that the same agent operates in a similar manner upon the animal economy possessed of its ordinary powers.

CCLXVII. When an individual or animal is in a state of stupor, the breathing is irregular or imperfect. This would seem to arise from the blunted sensibility of the cerebrum to impressions communicated in health by the nerves belonging to the function of respiration. Myexperiments have been made on rabbits; and I have observed, when these were stupified, that they appear for a while to forget to breathe, until the accumulation of blood in the lungs becomes painful, or the necessity of air imperative, The sensation arising from one of these 
causes is of an aggravated nature, and tends to arouse the breathing for a moment. The animal has sudden starts, and, after these, respires with regularity for a few seconds, but soon returns to its previous disordered condition.

CCLXVIII. The irregular, or interrupted mode of respiration, tends to render imperfect the proper oxygenation and distribution of the blood; and in accordance with these changes, we observe that the animal heat is diminished.

I have made many experiments on this subject, and have invariably found that the temperature is lessened as soon as the narcotic effects are manifest. The two following experiments may be regarded as affording a correct idea of the general changes which succeed the exhibition of opium. Thirty grains of this substance were injected into the cellular tissue of the abdomen of a full grown rabbit: its temperature before experiment was $102^{\circ}$.

$\begin{array}{ccc}\text { Time-Minutes. } & \text { Temperature. } & \text { Respirations. } \\ 40 & 96^{\circ} & 30 \text { per minute. } \\ 65 & 94 \frac{1}{2} & 33 \\ 105 & 93 & 33 \\ 160 & 96 & 40 \\ 215 & 96 & 40 \\ 295 & 98 & \text { More frequent. } \\ 7 \text { hours. } & 100 & \text { ditto. } \\ 13 \text { ditto. } & 102 & \text { ditto. }\end{array}$

Rabbit full grown; colour white; temperature $102^{\circ}$. About five grains of opium were injected as in the pre- 
ceding case, and the effects were equally well marked The experiment commenced at eight o'clock A. M.

Time. Temp.

Observations.

11 o'cluck A M. $92 \frac{1}{2}^{\circ}$ Affected with frequent and general spasms; paralysis of the lower extremites, and profound stupor present.

1 do. P. M. $\quad 91$ Spasms less severe, paralysis as before.

$\frac{1}{2}$ past 2 .

$\frac{1}{2}$ past 3 .

5 o'clock

$91 \frac{1}{2}$ Little alteration.

$90 \frac{1}{2}$ Ditto.

89

At this time the rabbit appeared very cold, and was evidently much worse. For the purpose of reviving it, I placed it near the fire, and, occasionally with my warm hand, endeavoured to stimulate the external circulation, by gently rubbing the surface of the body. These means gradually restored the animal to invigorated life. At 11 o'clock, P. M. its temperature had risen to 103. : scarcely any remains of paralysis. The rabbit was not altogether so lively as in health, but was inclined to eat, and partook of the food which was presented. It ultimately completely recovered. I have already stated that the eye is dull and languid in those cases in which the temperature is lowered and the blood imperfectly oxygenated. In the present rabbit, I had a good opportunity of verifying the correctness of the statement. It is well known that white rabbits have the iris of a red colour. During the experiment I was surprised to find that this entirely disappeared. If viewed at a 
short distance, it seemed black, but when examined close it was discovered to be of a dirty brown; and this colour continued, with slight alterations, for many hours after it had recovered the faculty of generating the usual quantity of animal heat. This fact is important, as it shows that the most delicate part of the capillary system is affected long after the thoracic organs have regained their general vigour. The eye may be considered an index of the condition of the whole capillary system,-and, therefore, it is important, as it teaches that the production of animal heat should be carefully attended to long after its re-establishment, because the unfavourable effects, occasioned by the circulation of improper blood, are not removed so soon as we might imagine.

The number of respirations is extremely variable. I have often counted 20 and 25 in one minute, and, almost immediately after, double this number. The cause of this was explained in CCLXVII.

CCLXIX. The respiratory function is disordered by the affection of the brain, and, as it recovers its ordinary sensibility, the proper oxygenation and circulation of the blood are established. But if the narcotic effects increase, the necessary conditions of the sanguineous fluid are no longer maintained; the extremities become cold, the surface of the body discoloured, the face tumefied, and the temperature diminished. These are the consequences of deranged respiration. 
The blood which is circulated at this time is dark, and but slightly stimulating. The contractions of the heart, and the vigour of the arterial system, occasionally observed under these circumstances, are not excited by the oxygenated properties of the blood as in health, but are continued in action, for a time, by the diffused and stimulating properties of the opium. If it were possible to roithdraw from the system the qualities which this poison has bestowed, it is not improbable that this deduction would immediately destroy the powers of life, because the blood would be deficient in its natural stimulus, then indispensable to promote its distribution.

CCLXX. If we suppose a given quantity of opium to be received into the system, whether from the stomach, cellular tissue, or rectum, it is gradually absorbed, and, in proportion as this is effected, the sensorial powers are disturbed, and this disorder is communicated to the respiratory function,-so that, while the former are directly, and the latter indirectly, influenced by the action of opium, the heart is frequently stimulated to augmented action by the same agent; but as the blood is deteriorated for want of those conditions by which it is perfectly oxygenated, and as its venous qualities accumulate, the artificial stimulus of the opium, if it does not act as a sedative from its inordinate quantity, is overcome by the sedative qualities of the blood increasing 
to a much greater extent the severe symptoms, such as diminution of sensibility and muscular motion.

CCLXXI. In those individuals who have died from the effects of opium, we remark no traces of inflammation; nor should we expect to find such, if we consider that inflammation can arise only from two general causes, viz. such as destroy or act violently on a part to which they are applied, or such as directly or indirectly tend to increase the oxygenated properties of the blood. The influence of opium lessens the natural stimulus of the blood, and acts principally on the nerves, increasing or diminishing the action of the organ to which they are distributed, and does not injure the texture like the class of acrid substances.

CCLXXII. Guided by the views already developed, it is not difficult to explain the different appearances observed in the examination of those who have died from the effects of opium. The lungs are found congested, occasionally presenting large dark patches; the heart full of black blood, or its vessels much engorged; the stomach, although not inflamed, exhibits greater vascularity than usual, and the veins of the thorax, abdomen, and brain are often found distended with dark venous blood. From the first stage of the narcotic effect, the sanguineous fluid has gradually lost its vital powers, assuming the character of venous; and when the heart ceases to 
be excited by the opium, its motions are too much enfeebled to propel the fluid to the distant parts of the body, and, therefore, they are insufficient to counterbalance the quantity of venous blood which returns to the chest. From this cause the blood continues to accumulate in the internal cavities, from the first symptom of depression till the complete extinction of life.

CCLXXIII. Without entering into a detailed account of the principles or plans of cure employed in cases of poisoning from opium, I shall endeavour to show the benefit that will be derived from attending to certain remedial measures suggested by the preceding observations. Vomiting is always recommended at the development of the first bad symptoms, for the purpose of removing the deleterious substance from the stomach, and thereby arresting absorption; but this effect has never been regarded in any other light than as ejecting the poisonous drug. If we keep in view what has already been stated in the foregoing pages, viz. that the internal organs are congested, a fact supported by every case related by ORFILA," we shall perceive the force of the following reasoning, and the correctness of the principles established in the present work-

By confining vomiting to the simple expulsion of the contents of the stomach, we lose the

* Vide Chapter IV. Class IV. Des Poisons Narcotiques. Traite des Poisons, Tom. II. 
most powerful agent that we possess for counteracting the baneful consequences induced by opium. I have proved by direct experiment on myself and another gentleman, that whenever vomiting is promoted, the blood is immediately more equally distributed throughout the system, and, as a necessary sequence, that the temperature of the body is increased, in accordance with the first principle, viz. " that animal heat is not in the direct ratio of the quantily of oxygen inhaled, but in the inverse ratio of the quantily of blood exposed to this principle."

CCLXXIV. It is therefore anvisable to employ vomiting in these cases as frequently as possible; it tends to relieve the congested lungs, and allows the blood to undergo more perfect changes, indispensable to overcome the injurious influence of the poison and the highly venous state of the blood.

CCLXXV. When an individual is in a soporose condition from the effects of opium, it is recommended that he be kept continually walking, as if this action were beneficial beyond the mere exercise of muscular power improving or maintaining the circulation, and exciting the respiratory function, by keeping the mind awake to the impressions made on the respiratory nervesas explained in ccLxvir. If, instead of adhering to these inefficient means, we promoted vomiting by medicines whose operation is not preceded 
by nausea, which would augment the evil, and if we placed the inferior half of the body in water as warm as the individual can bear it, employing at the same time almost constant friction,- - and if we also applied a bladder of warm water to the pit of the stomach, to lessen the congestion of blood in this organ and the thoracic viscera, we should so far equalize the circulation as to improve the chemical changes of the sanguineous fluid; and, in proportion as we accomplished this object, we should find that the powers which belong to organic and animal life readily return.

CCLXXVI. Depletion has occasionally been resorted to in the treatment of poisoning from opium, and has in some cases been found beneficial. Orfila, after making experiments on this subject, draws several conclusions favourable to its judicious employment, of which the following is one :

“ 2do, Qu'elle a paru utile dans quelques cir" constances, et même qu'elle a suffi pour retablir “ des animaux qui auraient péri si on ne l'eût "pas pratiquée." Depletion has been found useful in the cold stage of intermittent fever, because it takes off a part of the burden from the internal organs, and thereby permits the lungs to oxygenate more completely the blood, which speedily excites the action of the heart, the increased contractions of which distribute the blood more gene-

* 1bid.p 248. Tom. $1 \mathrm{I}$. 
rally throughout the system. When the constitution is labouring under the effects of opium, we have internal congestion of a similar kind, from a different cause, and to a less extent; but, if the remedy be beneficial on one occasion, it will on another, if the only difference between the two consist in the degree.

CCLXXVII. From observations contained in the preceding pages, it is evident that animal heat is diminished from congestion of the lungs and internal organs, agreeably to the principles fully stated in Chapter I. As this is demonstrated by direct experiment, the other views so intimately connected with these principles receive additional support.

CCLXXVIII. Observing the diminution of temperature and theirregularity in the distribution of the blood, from the narcotic effects of opium, it appeared to me probable that digestion would also be retarded. I attempted to show, in Chapter III., whenever animal heat was much lessened, and the circulation of the blood much disordered, that these states were incompatible with the secretion of gastric juice. The following experiments prove that, whenever the same or similar conditions of the sanguineous fluid exist, whether from the division of the par vagum, the application of cold, or the exhibition of opium, the same or similar consequences succeed. 


\section{EXPERIMENT I.}

In this experiment, opium was injected into the cellular membrane of the abdomen of a full grown rabbit. Before the operation its temperature was $105^{\circ}$. The experiment was commenced at seven o'clock, A. M.

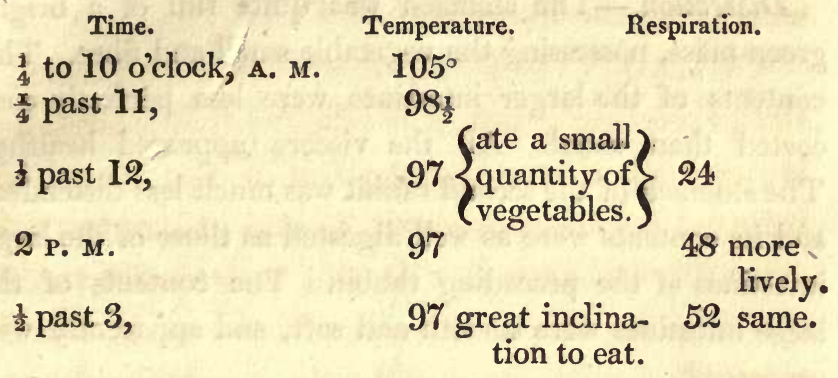

At this period it was killed, and another also which had taken food at the same time, that we might with more correctness compare the influence of opium.

Dissection.-Examination of the rabbit which had been subjected to the effects of opium. The stomach was full, containing a dark green vegetable mass, imperfectly concocted. The contents of the large intestines did not exhibit their ordinary changes; they were rough, and rather of a firm consistence; the other viscera were healthy.

The stomach of the sound rabbit was less distended than the other; but the mass was less perfectly digested, arising apparently from recent vegetable food which it had taken. The contents of the large intestines were smooth, soft, and completely changed in their vegetable character.

\section{EXPERIMENT II.}

Opium injected as before. The rabbit full grown. Temperature $104 \frac{1}{2}^{\circ}$. The experiment commenced at seven o'clock, A. M. 
Time. Temperature.

Respirations. $\frac{1}{4}$ to 10 o'clock, A. M. $104 \frac{1}{2}$ $\frac{4}{4}$ past 11. $100 \frac{1}{4}$

$\frac{1}{2}$ past 12.

2 P. M.

$\frac{1}{2}$ past 3, P. M.

$98 \frac{3}{4}$

$98 \frac{1}{2}$

$101 \frac{3}{4}$
26
44-more lively.
48-great inclination to eat.

The rabbit was killed at this time.

Dissection.-The stomach was quite full of a bright green mass, possessing the vegetable smell and fibre. The contents of the larger intestines were less perfectly concocted than usual. All the viscera appeared healthy. The stomach of the second rabbit was much less distended, and its contents were as well digested as those of the large intestines of the preceding rabbit. The contents of the large intestines were smooth and soft, and apparently well concocted.

\section{EXPERIMENT III.}

Opium injected as before. Temperature of the rabbit $102 \frac{1}{2}^{\circ}$. The animal full grown. Experiment commenced at half past eight o'clock, A. M.

$\begin{array}{lcc}\text { Time. } & \text { Temperature. } & \text { Respirations. } \\ 10 \text { o'clock, A. M. } & 102 \frac{1}{2} \circ & \\ \frac{1}{2} \text { past } 11, & 96 \frac{3}{4} & 24 \\ \frac{1}{4} \text { to 1. P. M. } & 95 & 40 \\ \frac{1}{2} \text { past 2, } & 96 \frac{1}{4} & 44 \\ \text { 4. oclock, } & 98 & 40\end{array}$

Killed at this time.

Dissection.-Stomach slightly distended with air. The vegetables which it had taken before the experiment had undergone scarcely any change. The contents were fibrous and coarse, presenting a chopped appearance. The colour, not uniform throughout, varied from a light to a dark green. The contents of the large intestines were 
rough, and by no means minutely divided as in those cases in which digestion had gone on well. The lungs were very much congested. The posterior surface exhibited dark patches and effused blood. From these well marked appearances, it was not thought necessary to kill a sound rabbit for the purpose of comparing the results.

\section{EXPERIMENT IV.}

'The rabbit full grown. Temperature $103 \frac{1}{4}^{\circ}$. The experiment conmenced at haif-past eight o'clock, A. M. Opium injected as usual.

Time. Temperature. Respiration.

$\begin{array}{lcc}10 \text { o'clock, A. M. } & 99 \frac{1}{2}^{\circ} & \\ \frac{1}{2} \text { past } 11, & 98 \frac{1}{4}^{\frac{1}{4}} & 44 \\ \frac{1}{4} \text { to } 1 \text { P. M. } & 100 & 64 \\ \frac{1}{4} \text { past } 2 & 100 & 40 \\ 4 \text { o'clock } & 100 & 52\end{array}$

Killed at half-past four o'clock, P. M.

Dissection.-The rabbits were not opened till ten o'clock next morning. The stomach of the one which had taken opium was very large, and slightly distended with air. The cardiac and pyloric orifices exhibited a vegetable mass of two different colours and states of digestion. At the cardiac the vegetable was little if at all changed, of a dark green colour, emitting a strong vegetable smell. The whole was covered over with a coating of gastric juice. At the pyloric extremity the vegetable had undergone a slight change, the green colour had almost disappeared, and the vegetable texture was less evident. The large intestines were distended with vegetable matter, presenting a rough, imperfectly concocted, appearance. The lungs were much inflamed at their posterior surface; the inferior parts of the lobes were very much congested and discoloured.

The sound rabbit, which was killed at the sametime as the preceding, was now opened. 
Disscction.-The stomach moderately distended with air. The vegetable smell was slightly evident. The contents of the cardiac and pyloric orifices were of the same colour and consistence, and were minutely divided. The large intestines were as full as those of the preceding rabbit ; but the digestive process had evidently produced great alterations on the matter which they contained. The lungs were a little more florid than usual.

These rabbits were fed at five o'clock, P. M. the day preceding the experiment, and were again fed an hour before the injection of the opium. They received no food afterwards.

CCLXXIX. It is unnecessary to multiply these experiments to prove that digestion is impeded when the temperature is lowered and the distribution of the blood deranged. "SpallanzaNI observed that the snake digested food faster in June, when the heat was at $82^{\circ}$ and $83^{\circ}$, than in April when it was only $60^{\circ}$; - from whence heconcludes that heat assists digestion."* Similar observations are found in the works of many naturalists who have paid particular attention to the influence of heat and cold on the powers of digestion. In making these experiments, I lost many rabbits from the operation of opium. It sometimes produces violent spasms, and when these occur soon after its exhibition the animals very frequently die. It is difficult to proportion the dose of the narcotic so as to produce the desired

\footnotetext{
* Observations on certain Parts of the Animal Economy, by John Hunter, p. 195.
} 
effects, without endangering the life of the animal. The influence of opium in these instances does not lessen the generation of animal heat, nor disturb the respiratory function to the same extent as the division of the par vagum in those cases in which the tube is not inserted into the trachea; and in accordance with this difference in degree, we observe that the changes in the stomach and intestines are more complete, and the lungs do not show the same traces of disease. I have noted the state of the respiration at the time when the animal temperature was taken; but the reader must not imagine that the number which is given is to be considered as a criterion of the character of the respiration prevailing for more than a few minutes or seconds, but merely as presenting to the mind an idea of the irregularity of respiration. Messrs Holroyd and Murrax kindly assisted me in these experiments.

R 2 


\section{CHAP. XII.}

\section{The Causes which Infuence the Action of the Heart.}

CCLXXX. There are probably few subjects concerning which a greater diversity of opinion exists, than regarding the cause of the contractions of the heart, and the precise manner in which these are influenced by chemical and mechanical changes. HaLLER and his partisans endeavoured to shew that the contractions are to be ascribed to a vis insita independent of the nervous system. LEGALLoIs concludes, that the heart derives its powers from the whole of the spinal cord: Wilson Philip has proved that this organ can act when the brain is removed and the medulla oblongata is destroyed,-a fact I have repeatedly ascertained. In Experiment V. page 48-9, the heart was found contracting 70 times per minute in a rabbit that had been inflated for an hour, in which neither brain nor spinal cord was present.

CCLXXXI. In Chapter X. we gave a cursory glance at the properties and office of the nervous system, and, therefore, shall not occupy the present pages in discussing the same point. The irritability which has been supposed by HaLLER 
to reside in and characterise muscular fibre is derived from the nerves of motion and sensibility. This power is very much modified by three causes : 1. The quantity of nervous influence possessed by an organ; 2. The disposition of the muscular fibres; 3. The nature of the stimulus necessary to develope it. The heart has been proved by ScarPa to be endowed with innumerable nervous fibrillæ, not confined, as was imagined by Soemmering and his predecessors, to the coats of the coronary arteries, but, on the contrary, intimately blended with the constitution of the fibre itself. If we consider the quantity of nervous power bestowed upon the heart by the sympathetic and par vagum, the disposition of the fibres and cavities of this organ, and, lastly, the energetic and constant stimulus operating here, we shall understand in what manner the contractions of an organ may be influenced.

CCLXXXII. There were two objections opposed to the opinion of HALLER. The first roas, for what purpose did the heart receive nerves? and the second, why was it influenced by strong emotions of mind? The first is a difficulty which was never satisfactorily solved by HALLER, and the latter is considered equally unanswerable by the opponents to the vis insita of this physiologist. Although I am fully persuaded that the contractions of the heart are to be ascribed to the nervous power distributed to the muscular fi. 
bres of the organ, yet I do not consider that this endowment explains the frequent and violent disturbance in the function of circulation observed in the exciting or depressing effects of passion. The abdominal viscera are said to be excited or depressed by the direct agency of mind, but the greater number of the illustrations brought forward in support of this opinion are not strengthened by any reasoning or facts that may not consistently be referred to causes intervening between the mental affection and the abdominal derangement. The development of views connected with this subject belongs to a subsequent chapter, and, therefore, we shall defer its consideration.

CCLXXXIII. Wilson Philip has satisfactorily proved, that the gradual removal of the brain, and destruction of the spinal cord, have little or no influence on the action of the heart; and yet it is evident, he observes, that it can be powerfully affected through the brain and spinal marrow by chemical and mechanical means. It is also stated by him and others, that it is extraordinary that the instantaneous destruction of the brain arrests for a few seconds the pulsations of the heart, since this organ continues its motions when the above nervous sources are entirely removed.

CCLXXXIV. This and many similar phenomena will, perhaps, become less mysterious by the 
following considerations and experiments. The sensorium, from its magnitude and the activity of its functions, must necessarily receive a great quantity of blood; and whatever tends abruptly to disorder its free transmission from the brain and its reception to this organ, will manifestly influence the contractions of the heart.

CCLXXXV. When the brain is destroyed by degrees, the heart is scarcely sensible of the gradual loss of blond, or of the effects it induces in the general sanguiferous system; but when the brain is instantaneously crushed, we have other consequences. This operation gives rise to three results, which go far to explain the momentary cessation in the contractions of the heart.

First, The blood which is propelled along the arteries in its passage to the brain is suddenly forced back upon the heart, or is stopped in its course, and thereby prevents the circulation of the next portion transmitted by the contractions of the heart.

Second, The circulation of blood in the veins is equally disturbed. When the nervous mass is suddenly compressed, the blood that was previously flowing regularly to the heart is sent either with inordinate force, as it is in this direction alone it can escape, or its circulation is complelely stopped; it is probable that both conditions with modifications occur. 
Third, The blow which is given necessary to produce the desired effect shakes the rohole system of the animal. The circulation of blood in the larger arleries and veins is for a moment retarded, if not completely arrested.

CCLXXXVI. If the circumstances which have now been stated be taken into consideration, it must be acknowledged that the mystery connected with the phenomenon, if not altogether unravelled, is nearly so ; and in reasoning on the manner in which the heart recovers its contractions, we shall find the present view materially supported. In a few seconds after the blow, the heart commences to pulsate, at first feebly, but in a few minutes with almost its ordinary vigour. During the short period of its inactivity, it is either gradually and almost imperceptibly overcoming the burden by which it is oppressed, or during this time the whole sanguiferous system re-establishes its regular functions, and therefore we have the contractions of the heart as soon as the different relations existing between the various parts of this system are restored.

CCLXXXVII. If it were owing to the suppression or injury of the nervous influence, the cessation ought to be rather of a permanent than evanescent character, because one of the sup. posed sources of nervous power is destroyed. 
CCLXXXVIII. Wilson Pfillip has endeavoured to ascertain in what manner the action of the heart is influenced by the application of chemical and mechanical agents applied to the brain; and he brings forward a great number of experiments, which prove, that these occasionally act powerfully on the motions of the heart. $\mathrm{He}$ says, that these act through the nervous system. I shall attempt to shew from his own experiments, and from others which $I$ have performed, that it is more than probable that the effects are communicated through the sanguiferous.

CCLXXXIX. In applying spirits of wine or opium to a small portion of the brain, no effects, he says, were produced; but as soon as these were diffused over a large surface, the action of the heart was affected. If we augment the surface to which one of these agents is applied, we include in the same proportion a greater number of absorbents or capillary vessels of what kind soever, and therefore the action of this organ is more liable to be influenced, because a greater quantity of the stimulus or sedative is absorbed and transmitted to the heart, on whose nerves it produces its peculiar effects. The greater number of the subsequent experiments demonstrate the correctness of this opinion. Regarding the circulation as the medium of these agents, and the heart as influenced by the matters which the blood had absorbed during the circulation, I 
stated to my friends who kindly assisted me, that it was not improbable we should observe the same results on employing chemical means, in one instance, on a small portion of the brain, if this were in the vicinity of the larger vessels transmitting the blood to the chest, that we observe in another if the portion be much more extensive and confined to the surface. To prove the truth of this opinion, I had a small tube made about three inches long; the aperture of the superior end was half an inch in diameter; that of the inferior, applied to the base of the brain, was one-eighth of an inch. When this tube was introduced, the smaller end was made impervious, until it had reached the base, by a slender piece of wood, in order to prevent the brain from filling the tube, which it had previously done in some of my earliest experiments, a circumstance which tended to retard absorption, as the contents of the instrument had lost all connexion with the brain, and become as inorganic matter. Adult rabbits were employed in this series of experiments.

\section{EXPERIMENT I.}

The animal being made insensible by a blow upon the occiput, the chest was opened, that we might observe the contractions of the heart; and afterwards a small portion of the skull-cap was removed, to allow the introduction of the instrument. A few drops of prussic acid were then 
applied. The heart contracted, but with an enfeebled motion, for 45 seconds, and then ceased, but recommenced in eight or ten seconds, and continued to contract five or six times per minute for the space of three minutes, at which time it altogether ceased.

\section{EXPERIMENT II.}

The preparatory steps of the operation were the same as in the preceding instance. A few drops of prussic acid were applied. The contractions of the heart were less frequent, but appeared stronger directly on the application of this poison. The results were not very decisive. In three minutes from the commencement, the contraction still remaining, a few drops more were applied, and the heart immediately became affected; in a few seconds it gave scarcely a perceptible pulsation.

\section{EXPERIMENT III.}

The preparatory steps having been attended to, a few drops of the spirits of wine were applied. The motions of the heart were more frequent for a few seconds, but as soon became slow. Infusum tabaci was then employed; the contractions were quickened for a few seconds, but almost immediately became much slower. Prussic acid was lastly applied; and this, like the preceding agents, seemed to quicken the contractions, but in a few seconds after its application the heart ceased to pulsate.

\section{EXPERIMENT IV.}

In this experiment infusum tabaci was employed first. On its application the motions of the heart became almost instantaneously slow; they were previously strong and frequent. While the contractions of the heart were feeble and 
slow, a few drops of alcohol were applied, which immediately increased their frequency.

\section{EXPERIMENT V.}

- Opium was applied first in this experiment. The contractions of the heart became immediately rapid and irregular, and continued for $\mathbf{2 0}$ seconds; in 10 seconds more they became slow, and were observed to be in this state for 40 seconds, at which time spirits of wine were applied, but did not appear to produce any very obvious change, except that the motions were converted from their former irregular character into one somewhat undulatory.

\section{EXPERIMENT VI.}

In this experiment prussic acid was applied first. In 80 seconds from the application the heart had almost ceased to contract. Alcohol and opium were afterwards employed, but did not produce any obvious effects.

'To show still more satisfaetorily that the nervous system has little, if any influence, in transmitting the effects of the different agents to the heart, the subsequent experiments were performed. If this can be shown, it necessarily follows, that the small or extensive surface, alluded to by Wilson Prilip, is to be considered as merely presenting different degrees of facility for the absorption of what is applied to the brain.

\section{EXPERIMENT VII.}

The animal being made insensible, we cut down upon the lumbar vertebrae ; and, having separated two of them, a heated iron was passed from this point as far as the cervical, and was allowed to remain there during the experiment. The parietes of the abdomen were then divided 
immediately beneath the diaphragm, in two opposite directions, until the scalpel touched the two sides of the spine, for the purpose of destroying all lateral connexion. When this operation was finished the bowels were removed, and a few drops of the spirits of wine were applied to the vena cava immediately above the pelvis. Before the application of these the beatings of the heart were almost imperceptible, but in a little less than a minute they acquired comparative frequency and force for about 40 seconds.

\section{EXPERIMENT VIII.}

The animal being made insensible, the chest was laid open, that we might observe the motions of the heart. The jugular vein was then opened, and two drops of prussic acid were introduced through a blow-pipe : the contractions of the heart instantly ceased. I have performed this experiment several times; the results were always evident, but never so obvious as in this instance.

\section{EXPERIMENT IX.}

In this experiment it was intended that the brain should have been removed and the spinal cord destroyed. The former was accomplished with a greater loss of blood than usually occurs, owing to an accident attending one of the ligatures; and two of the lumbar vertebrae were separated in order to introduce the heated wire; but every wire we had provided was found too large; therefore, in this experiment, the brain alone was removed. The chest being opened, as before, an aperture was made in the abdomen to allow the operator to lay aside the intestines. A few drops of the spirits of wine were applied to the vena cava, as in Experiment VII.; the heart, for a few seconds, had its motions accelerated, but these, in a few minutes, completely ceased. 


\section{EXPERIMENT $\mathrm{X}$.}

This rabbit was decapitated, and the spinal cord was destroyed by means of a heated wire. A few drops of the spirits of wine were applied to the vena cava, as in the above case. The contractions of the heart were quickened, and, after a few seconds, when the increased action had subsided, a few drops more were applied to the vena cava immediately before it enters the thorax : the contractions of the heart were again affected, as in the first instance.

CCXC. The above experiments prove, that the extent of surface is to be considered as merely facilitating absorption, and not as presenting a greater mass of the nervous system to the influence of the agents we employ. If we diminish the surface and increase the facilities of absorption by applying the materials near the large veins at the base of the brain, we have effects equally well marked as when we occupy an extensive surface of the organ. Wilson PHILIP observes, "It is evident that the heart not being subject to stimuli, whose action is confined to a small portion of this organ, and being equally affected through all parts of it, must render it much less subject to irregular action."* If the heart be subject to the whole brain, it will be capable of being excited or depressed by a concentration of influence to any single part; and this was proved in a very satisfactory manner by some of the preceding experiments, In Experi-

* Wrison Philip, p. 117. 
ments V. and VI. the effects were almost instantaneous. The heart therefore is not protected by being subject to the whole brain, but because the sensorium has no direct influence through the nervous system upon the action of the heart.

CCXCI. If the heart were influenced by the whole brain, in the sense understood by some physiologists, we should naturally expect, when the rohole of this organ was removed, that the action of the former would be materially affected, rohich is not the case. If the connexion of these organs be regarded as simply nervous, and of that extensive description that the nerves of the heart derive their power from every part of the brain," the possibility of completely destroying this relation without injuring the contractions of the heart, is completely at variance with such an opinion.

CCXCII. Fontana destroyed the brain and spinal cord of 24 frogs; the same organs in the same number of frogs were left entire. The 48 were made to swallow a certain quantity of opium, for the purpose of observing whether the action of the heart would be more influenced in the one set than in the other; but there was no difference in the results. Monro endeavoured to affect the limb of a frog through the sciatic nerve, which was isolated, but did not succeed. $\dagger$

* Wilson Prilip, p. 118.

+ Essays and Observations Physical and Literary, Art. XIII. p. 295. Vol. III.-Many experiments have been performed in 
This distinguished anatomist also proved, that opium produces its effects when all the nerves of a part to which it is applied are cut out.

CCXCIII. It has been shown by different physiologists, that mechanical means, such as pressure, either partial or general, applied to the brain, disturbs the regular motions of the heart; and this fact, instead of supporting the views of Wilson Philip in regard to the nervous connexion existing between these organs, will be found, if examined, to strengthen the principles proposed, in which I endeavour to prove, that the circulation is the only medium through which these derangements are communicated. The heart has been shown to be independent of the brain, yet capable of being influenced through it. If the former organ be independent of the latter, the sensorium is every moment dependent upon the heart for its vitality and the exercise of its functions; therefore, whatever mechanical means are applied, whether to the whole or any single part, will consequently disorder the action of the heart in proportion to the extent of the sanguiferous system implicated. The return and distribution of the blood soill be retarded or arrested; and this circumstance

order to ascertain the action of opium and other substances upon the animal economy since this able article was written ; but I am much disposed to think that we have gained scarcely one sure step for the last half century, at the commencement of which it was published. In reference to this subject we may employ the words of a German anatomist, "that what is new is not true, and what is true is not new." 
cannot occur, roithout giving rise to evident effects in the contractions of the heart.

CCXCIV. Pressure cannot disorder the motions of the heart by deranging the nervous fluid in its flow to this organ, because the action of this is slightlyaltered by the destruction of the cerebrum itself in which this supposed agent is annihilated; neither can it be considered precisely in the light of an irritant, because the hemispheres of the brain, which may be particularly acted upon, have no obvious and direct connection with the nerves which are distributed to the heart ; and, moreover, the application of pressure to a pulpy mass does not possess the qualifications necessary to disturb the remote relations of the nervous system by the irritation it produces. But pressure, and all mechanical means, are well calculated to occasion immediate disturbance in the general circulation of the system, particularly in the contractions of the heart, from the direct and close communication existing between this organ and the brain.

CCXCV. Monro,* and Wilson Philit, ascertained by experiment, that a solution of opium poured into the cavity of the abdomen produced more speedy effects than the same applied to the mucous membrane of the intestines, or almost any other part of the body. The former imagines this circumstance to arise from the nerves * Id. opus, p. 14.5. 
belonging to the peritoneum or intestines being affected ; the latter brings forward a contrary opinion, supported bydirect experiment, in which he attempts to show, that opium applied to the coats of the blood-vessels destroys their muscular power, and therefore must " influence the motion of the heart, by impeding, or entirely interrupting, that of the blood, in nearly one-third of the whole animal."**

This explanation is very ingenious, but far from being correct. He supposes that the heart becomes immediately affected from the muscular power of the capillary vessels being destroyed, preventing the usual quantity of blood being transmitted to the heart. To shew that it does not, by any means, depend on this cause, I removed the intestines in Experiments VII. IX. and X. and applied a stimulus to the vena cava, just above the pelvis, and found that the motions of the heart were almost instantaneously quickened,-and, still further, that the effect in some degree was proportionate to the vicinity of the vein acted upon to the heart. By applying the agent to large vessels, we cannot suppose we either paralyse or stimulate the coats, so as to affect indirectly the circulation; and therefore the stimulus or sedative must be conveyed by the absorbents or blood-vessels.

* A Treatise on Febrile Diseases, Vol. IV. p. 685. 
CCXCVI. The correctness of Wilson Philip's explanation rests on the supposition, that nearly one-third of the blood of the system is prevented from flowing to the heart; but if the opium influenced merely the quantity of the vital fluid, and did not diminish the irritability of the heart, by being carried to this organ by the circulation, its contractions would continue much longer, and would be less sensibly retarded by the application of such means.

CCXCVII. Galvanism is one of the most powerful stimulants we possess, and when its mode of action on the animal economy is well understood, many diseased and deranged states of the system will be alleviated or removed by its application. This agent has been frequently employed to prove that the heart can be excited through the nerves distributed to its muscular substance; and the following Experiments will show that this effect is as readily produced by applying the positive or negative wire to the vein, or almost every other part of the body, as to the nerves themselves. In these Experiments two troughs, each of 50 two-and-a-half inch plates, were used. Adult rabbits were always employed.

\section{EXPERIMENT I.}

The negative side of the battery was connected with the heart, and the positive with the jugular vein. The heart was immediately affected; its enfeebled contraction was changed into an imperfect vermicular motion. The posi- 
tive wire was afterwards brought in contact with the vena cava in the abdomen; the heart was again excited, but did not present the same peculiarity.

\section{EXPERIMENT II.}

The negative wire, in the preceding experiment, was in contact with the heart, the positive was connected with the mucous membrane of the nose; the contractions of the heart were instantly renewed. The same effect was observed on the positive wire being placed in the substance of the brain.

\section{EXPERIMENT III.}

Galvanism was employed as in the two preceding examples, but the results were scarcely perceptible. This is probably to be attributed to the loss of nervous irritability, - the contractions of the heart having been previously arrested by prussic acid applied to the brain, and to the heart itself.

\section{EXPERIMENT IV.}

In this experiment the positive wire was connected with the heart, and the negative brought in contact with the mucous membrane of the nose. The motions of the heart were instantly renewed, characterised by the vermicular contraction previously alluded to; the negative was then connected with the par vagum, and subsequently with the jugular vein, at all times isolated, and the same effects occurred.

\section{EXPERIMENT V.}

In this experiment the wires were placed as in the preceding, and the results were the same. A curious circumstance occurred in this experiment, which is worthy 
of being mentioned. While the positive wire remained connected with the heart, Mr KeMP was busy in applying the negative to different parts of the body. He at last placed it in contact with the external surface of the stomach, which occasioned no contractions, probably from its being rather dry; but, either accidentally or intentionally, he penetrated the parietes of this organ, which produced violent motions of the extremities, diaphragm, and heart. He inquired if there were not a nervous coat which would explain the phenomenon. Mr Chester answered him in the affirmative, and at the same moment laid it bare; but when the wire was brought in contact with this nervous coat, no contractions followed its application : they returned with the same violence when the wire penetrated the parietes of the stomach. I proposed that the food should be exposed, which was done, and when the wire was placed in the halfdigested contents, the motions of the extremities, diaphragm, and heart became as evident as in the first instance.

\section{EXPERIMENT VI.}

Having proved, by the preceding experiments, that the motions of the heart and other parts of the system could be excited equally through nerves, mucous membranes, veins, and the food in the stomach, I suggested a further modification. The heart, after having illustrated the above views for about a quarter of an hour, was entirely separated from the body, and isolated by means of glass; a piece of thread was then tied to a small portion of the aorta connected with the organ, and was afterwards moistened. When the positive wire was in contact with the heart, and the negative with the thread, or when reversed, the contractions of the heart were again manifest. These 
were not characterised by an almost imperceptible motion of a few muscular fibres, but were accompanied by the simultaneous contractions of the different cavities.

\section{EXPERIMENT VII.}

In this experiment the positive wire was in contact with the heart, and the negative alternately with the par vagum, jugular vein, vena cava, mucous membrane of the nose, and food in the stomach,- and precisely the same results took place as have been already stated.

\section{EXPERIMENT VIII.}

The heart being removed from the chest, and isolated as before, a thread was tied round the remaining portion of the aorta. The positive wire was connected with the heart, and the regative with the thread, previously moistened. The contractions in this experiment were more evident than in the preceding examples. The free end of the thread was afterwards placed in the centre of the food, and at the same time that the negative wire was in contact with the contents of the stomach, the positive still remaining in connection with the heart, the contractions of this organ were again as manifest as if the galvanic power had passed through the par vagum, or directly through the thread.

Mr Chester, Mr Kemp, and Mr Hardy, assisted at all the experiments contained in this chapter, with the exception of Experiment IX. p. 269, and Mr Brooks, Dr Savage, and Captain Brown, the Conchologist, were present at several of the experiments.

From the preceding experiments and views, we are authorised to draw the following conclusions : 
1. That the heart is influenced by mechanical agents of every description applied to the brain, in proportion to the disturbance induced in the sanguiferous system.

2. That the heart is influenced by chemical agents applied to the brain, according to the extent of surface, or cerebral substance occupied by the individual agent, as the absorption of the same is regulated by these conditions.

3. That the heart is affected in degree according to the strength and quantity of chemical means employed, and the vicinity of their application to it.

4. That the heart can be influenced by galvanism equally well through nerves, veins, and arteries, through the substance of the brain, mucous membrane of the nose, food in the stomach, and moistened thread; indeed the effect produced appears independent of any organic inherent quality.

5. Those parts of the animal frame appear the most powerfully excited by galvanism which seem to be endowed with the greatest quantity of motory nerves, viz. the extremities, the heart, diaphragm, and muscles of the face.

6. That the heart is not protected from irregularity because it derives its power from the rohole brain, but because it has no nervous or direct connexion with the cerebral mass of the hemispheres through which it can be readily affected.

7. It would also appear probable, that the irritability or motory property of the heart was not materially affected by the destruction of the brain and spinal cord, as its contractions were found to continue long after their removal, if the circulation of the blood were maintained by inflation. This circumstance is extremely favourable to the opinion expressed in the pre. ceding inference. 


\section{CHAP. XIII.}

\section{On Palpitation.}

SEct. I.-The General Causes which produce this Affection.

CCXCVIII. WE have scarcely a slight cough or pain, if the pulse be quick or small-no uneasiness of stomach, shooting or fixed pain of headno heaviness or lightness-palpitation of heart, or irregular pulsation in any part of the system, but now comes within the definition of a nervous affection and the treatment of a nervous principle. The idea of a nervous power has had great influence in the treatment of diseases of the heart. When indications of practice were not well marked, or did not appear very urgent, we have too frequently disregarded them as mere nervous derangements, till, from neglect, they have demanded prompt and active measures, at a time probably when our means allow us to palliate only.

CCXCIX. Palpitation occurring in the nervous temperament is characterised by its quickness, and the gentle impulse which it gives; the chest is not elevated by its action ; the pulse is frequent and small, and the animal heat scarcely at all changed. 
CCC. In individuals of a purely nervous constitution, the parietes of the heart, its valves or vessels, are seldom much disorganized; but patients who have been long troubled with such complaints are remarked to die frequently of Phthisis ; and in those cases in which we cannot discover the efficient cause of death, we observe, on dissection, the traces of chronic disease of the thorax and abdomen, such as congested or tuberculous lungs, indurated or enlarged liver, vascular or thickened appearance of the stomach, or adhesions in some parts of these cavities. We shall now investigate the causes which determine palpitation, and endeavour to explain the various kinds of disorganizations, either co-existent with, or subsequent to such an affection.

CCCI. An individual, from the preponderance of the nervous temperament," is prone to receive slight external impressions; shadows seem tangible; ordinary sounds are deemed anomalous or strange, and the different senses appear to acquire additional acuteness. The continued operation of these causes predisposes the system to organic changes, or functional derangements. The frame is emaciated, the cheek loses the glow of health, the mind its usual buoyancy, and the appetite becomes fastidious. The mental anxiety which he suffers, the inactivity of

* I continue the use of this term, as it conveys to the mind of the reader the character of the constitution particularly predisposed to palpitation. 
life which he leads, the neglect of proper food, and stimuli, such as society, fresh air, and the influence of pleasurable objects, have one general tendency, to change the equality of the circulation. The influence of these causes, from the first, has been to determine the blood upon the internal organs, as proved by the paleness or otherwise unnatural complexion of the countenance and surface of the body, the great sensibility to cold, the want of appetite, and the irregularity of other organic functions. I have previously stated that this condition of the sanguiferous system invariably tends to diminish the stimulating properties of the blood; and, from the combination of these causes, we shall be enabled to give reasons for the occasional occurrence of palpitation.

CCCII. The lungs and the heart are intimately connected by the nature and proximity of their functions. If the heart, from malconformation, be not capable of propelling the blood which the lungs transmit, syncope or death is the consequence; or if the lungs, on the other hand, send blood deficient in its ordinary qualities, the pulsations of this organ become weak, cease, or exhibit convulsive action; or if it be unusually oxygenated, its contractions are forcible and frequent.

CCCIII. In the nervous constitution, when the heart is troubled by occasional palpitation, the lungs, in common with the rest of the inter- 
nal viscera, possess more of the sanguineous fluid than is consistent with the proper performance of their office; and, therefore, the connexion between these important organs is rendered highly susceptible to slight alterations occurring in the general circulation. Mental emotions, fatigue of mind or body, or internal functional disorders, by disturbing still more the quantity or quality of blood transmitted from the lungs to the heart, derange the contractions of the latter organ. If the quantity of blood which is sent to the heart be greater than usual, its powers are oppressed, or, from the urgent circumstances in which it is placed, it is unable to circulate the blood it receives with ordinary regularity, and its convulsive motion or irregular action is the consequence of this inability. Palpitation, in such instances, arises therefore from the general cause, an overcharged state of the lungs.

CCCIV. In the nervous temperament the pulse is frequent and small. The heart contracts in frequency and force, cceteris paribus, proportionately to the degree of stimulus which the blood possesses. In this state of the system the blood is deficient in its usual stimulating qualities from the causes mentioned in CCCI. Consequently, the heart neither dilates nor contracts so fully as in the state of health : but the necessity of propelling the blood is great; it is, therefore, compelled 
to accomplish, in frequency and feebleness, what it sometimes performs in slowness and energy.

The causes which have been mentioned as having the general tendency to determine the blood. upon the internal organs, and which alteration is also invariably accompanied by a deteriorated condition of the blood, affect, in the first place, the different viscera with derangements, the continuation or severity of which ultimately gives rise to the various disorganizations observed in the lungs or abdominal organs.

CCCV. Having briefly investigated the manner in which the internal organs are disordered by mental emotions, fatigue of mind or body, or other depressing causes, we are now prepared to understand in what way these derangements are removed by exercise, fresh air, lively society, and other kinds of amusements. Muscular exertion, and the cheerful feelings of the mind, promote the better oxygenation and circulation of the blood. The former equalizes the distribution of the vital fluid, and this change diminishes the proportion existing in the lungs, and the beneficial results which follow are in accordance with the principles of the first chapter: the latter improves the inspiratory or irregular character of the respiration.

CCCVI. In infancy and childhood, palpitation seldom occurs. At this age, every organic function is active ; respiration is more frequent 
the pulsations of the heart are more numerous and regular than at any subsequent period. From the force of the circulation, the energy of each organ, and the unclouded serenity of the mind, there are few causes capable of producing an overcharged state of the lungs.

CCCVII. Palpitation of the heart occurs very frequently in the constitution of the female at two very different periods of life. On the development of the uterine functions, the circulation of the blood undergoes great changes; new demands are made, and the regular course of the vital fluid is directed to other organs. But these important alterations are not immediately established, and, until they are, disorders in the circulation, disturbing the motion of the heart, are extremely liable to occur. When these organs are fully formed, the delicacy of their functions is subject to the influence of many circumstances, all of which act powerfully on the distribution of the blood. At the latter period of life, the demands of these parts of the system are diminished. The blood, which has for a series of years been determined here to supply the activity of functions unessential to life, is now no longer required: It is therefore the intention of Nature to diffuse the quantity appropriated to these functions equally throughout the system; but the attainment of this object is occasionally opposed, or 
rendered imperfect by a variety of causes calculated to derange the sanguiferous system.

CCCVIII. The application of cold has the tendency to determine the blood upon the internal organs; and if palpitation be the consequence of its influence, this arises from an overcharged state of the lungs.

Sect. II.-Organic Causes which produce Palpitation.

CCCIX. WhrN we examine the organization of the heart-the delicate adaptations of its valves-the constitution of its cavities-the continued action of its parietes-and the variety of vessels with which it is connected,-we are in some measure prepared to expect that a multiplicity of causes will tend to disorder its function by the disorganization occasioned in the component parts of its structure.

CCCX. The organic diseases of the heart, and those of other organs that produce palpitation or irregular action, are divided into three general classes, -

1. The first class includes the organic diseases of the heart itself. These, although differing in nature, situation, and extent, are classified, from possessing one common tendency, to obstruct the natural current of the blood through the cavities of the heart. 
2. The second class includes thoracic and abdominal affections, diseases, and injuries of the spine, and medulla oblongata,-which are also classified, from possessing one common influence on the organs of respiration.

3. Tumours and many anomalous diseases, which seem to act directly upon the larger vessels of circulation, constitute the third class.

CCCXI. The first class is extremely extensive. For the purpose of facilitating description, and of shewing more clearly than I otherwise could the consistency of the classification, it is subdivided as follows :-

$a,-a$ small heart.

$b,-$ one or more of its cavities enlarged or diminished in capacity.

$c$,-diseases of the valves proper to the heart and the great vessels with which it is directly connected.

$d$, -inflammation.

$e,-$ adhesion of the pericardium.

$f$,-imperfect organization of the heart.

CCCXII. The heart may be perfectly well formed in relation to itself; its cavities, valves, and parietes, may be proportionate to each other ; yet, as a whole, its relation to the system may be inaccurate, and therefore may predispose to occasional irregularity of action. 
CCCXIII. During the state of repose, when the circulation is equable or little invigorated, such a heart may perform its office ; but if muscular exertion be employed, or powerful internal stimuli be taken, or if the mind be excited by violent passion, more blood is transmitted in a given time than it is capable of receiving or propelling with its accustomed regularity. This peculiar conformation may sometimes be the cause of palpitation on occasions apparently unimportant. It will make the heart extremely liable to suffer from an overcharged state of the lungs.

CCCXIV. The heart, as a whole, may, in its relations to the system, be exact, yet its parts may want harmony among themselves. If we were sufficiently advanced in knowledge to estimate slight deviations of this kind, it is not improbable that many symptoms, otherwise inexplicable, would be found to originate in such a conformation. When a ventricle or auricle is evidently enlarged or diminished, we are almost sure tofind this accompanied byconsequences that cannotbe referred to any other cause than an increased or diminished capacity; and it is not improbable that the predisposition of some individuals to occasional palpitation is sometimes to be attributed to this cause. When a cavity is lessened, which sometimes occurs, its parietes, for themost part, are considerably thickened, and this condition consti- 
tutes one form of hypertrophy. If one cavity be less, from disease, than it ought to be, it is clear that its diminished capacity cannot receive the same quantity of blood. If the seat of this malconformation be the right auricle, every motion of the body that facilitates circulation will be apt to derange or destroy the function of the heart; or suppose the right ventricle to be dilated, the contraction of the auricle will send its usual quantity of blood, but a portion of this will, of necessity, regurgitate; and, if the latter cavity be much excited, it will send more than it is accustomed to transmit, and, in this way, it will hasten the effects called palpitation, or irregular action of the heart. This phenomenon occurs because the augmented cavity is frequently accompanied by debility. The thin parietes in passive aneurism do not present the same quick sound to the ear, the same strong impulse to the hand, nor the same frequent and bounding pulse that are observed in active aneurism. Palpitation in both conditions is produced by the same exciting causes. Whenever a quantity of blood, greater than what is natural, is transmitted to the dilated ventricle or auricle, its attenuated parietes are inadequate to the task of propelling it, the consequence of which is, that the fluid accumulates, and excites the heart to convulsive or irregular action. SExac and others have imagined, that the cavity being larger, it must necessarily be more stimulated, as it possess= 
es more blood. This reasoning is evidently fallacious. If we suppose the enlarged cavity to receive an ounce of blood, instead of half this quantity in its natural condition, each superficial particle of this fluid, as in ordinary cases, will be applied to a given point of the internal surface, and will act on this point as if the cavity were only half the size. The contractions of the heart may be regarded as made up of an infinity of smaller motions, arising from the innumerable particles of blood: therefore, one cavity twice the size of another, supposing equality in every other respect to exist, cannot possess a greater proportional stimulus, or exercise a more energetic action. If it contained a greater proportional quantity of blood, and were on that account more excited, the muscular fibres would exhibit greater strength in their contractions; but these are slow, and feeble even in the state of palpitation, compared to those of the naturally organized heart when inordinately stimulated.

CCCXV. Diseased valves may be regarded as the cause of almost every change of structure that affects the heart ; their extreme delicacy disposes them to suffer from every irregularity of action in the sanguiferous system; and when disordered, their importance makes them readily produce derangement of function, or structural disorganization in other parts near and remote. If they become cartilaginous or ossified, or if 
they diminish or increase the communications between two cavities, the heart will be predisposed to palpitation.

CCCXVI. Vegetations, and all kinds of accretions, may probably be looked upon as simply lessening the cavity, and placing this in circumstances somewhat similar to the one whose capacity is diminished by a thickening of its muscular parietes.

CCCXVII. Inflammation of the heart is fortunately a rare disease. If well marked in its symptoms, it often terminates in death, or occasions chronic affections of the lungs or great vessels. The peculiar feature in this disease is, that the irritability of the heart is increased, and is violently acted upon by the presence of blood possessing, for a short time, its usual qualities. The derangement of the circulation, and the sufferings of the patient, promote the respiratory function; the blood becomes better arterialized, and this irritates still more the sensibility of the inflamed organ.

CCCXVIII. Ossification of the coronary arteries has not been included in the subdivision of the first class, because I am rather inclined to believe that its importance has been exaggerated. Angina Pectoris, which it is said to occasion, has too frequently been found to occur, in all its severity, without the least trace of ossification, to allow us to suppose that this condition of the 
vessels is necessary for its production; and it has also been observed, that ossification of the coronary arteries has been detected in individuals who were exempt from every symptom of the disease.

If ossification has any effect upon the heart, it must diminish the freedom of its action, either by lessening its nourishment or by destroying the contractility of its muscular fibres. Whether the muscular fibres of the heart or its vessels be disorganized, the valves diseased, or the openings between the cavities enlarged or diminished, or whether the great vessels connected with the heart be affected, or the proper proportions between the cavities be wanting, a paroxysm will be excited whenever a greater quantity of blood is transmitted in a given time to the organ than its condition is able to receive or propel.

CCCXIX. Burns, in his Treatise on the Diseases of the Heart, quotes the opinion of BELL, as apparently explaining the manner in which a child overcomes or shortens a paroxysm of the Cærulean disease, which presents the same general symptoms as Angina Pectoris, except in certain external signs, which depend on a difference of the organic cause that induces this state of Asphyxia. He says, "the patient lessens the capacity of the chest, if he be very young, by turning on his belly, or if he be older he presses his breast firmly against a table or any solid ob- 
ject," that he may expel "portion after portion. of the contaminated air, as will in some measure restore the balance of arterial and venous blood." If this opinion be examined, it will be found incorrect. It is not the bad air which the chest contains, nor the good which it wants, that prolongs or would relieve the paroxysm. If the patient could receive oxygenated air, and if this stimulated as it does in a natural inspiration, it is not improbable that the life of the individual would be destroyed. The introduction of fresh air would accelerate the circulation, and send a still greater quantity of blood to the heart, which ceased, and ceases to act, because it had, and has, more than it can circulate. During the Aspliyxiated stage of the paroxysm, the heart, in the greater number of instances, is operating slowly with out the dangerous assistance of the lungs; but as this is only to a small extent, it requires some time before it can relieve itself of its accumulated burden : when it has accomplished this object, inspiration succeeds, and the stimulus of the air is then beneficial, as it tends to establish the harmony which must subsist between the respiratory and the circulatory systems.

CCCXX. The efforts which the individual makes are instituted neither to admit pure nor send out impure air, The patient, whether sensible, or only obscurely so, during the paroxysm, invariably acts from sensations. The congestion 
of the lungs is accompanied by painful states of the mind, and, without being directed by any ideas of his own, he follows the impulse of his feelings, and these will give rise to his pressing the chest, or rolling upon his belly, as it is in these regions that the pains have originated, and in these in which they are continued.

CCCXXI. I am not aware in what manner adhesion of the pericardium to the heart produces palpitation; that it does so, is not improbable. Ad. hesion is so frequently connected with this organ, without its presence being indicated by such an affection, and when this irregular action has been found co-existent with such a disorganization, there has been likewise so much partial or general disease, that the difficulties deter me from forming even a conjecture on the subject.

CCCXXII. Imperfect organization of the heart is well illustrated by the Cærulean disease. Burns, in speaking of this affection, observes, that part of the blood passes through the foramen ovale into the left auricle; and when the ductus arteriosus is also pervious, another portion is transmitted from the pulmonary artery to the aorta;-the third and remaining quantity circulates through the lungs in the usual way. The blood in the two former divisions undergoes no change, but mingles with the arterial in the situations in which it terminates. This account is perfectly correct. He then remarks, "Now, from this 
short statement, it must be obvious, that, sooner or later, an accumulation of blood, highly venous, must take place in the arterial system; and whenever this proceeds a certain length, we are prepared to understand why the body feels weak; why the pulse flutters and the heart vibrates; why the vital functions are nearly arrested; and why the person expresses the greatest anxiety and oppression in the chest, referrible in part to the presence of venous blood in the left side of the heart, but occasioned equally by the consent which obtains between this organ and the lungs. Such is a general explanation of the cause of the occurrence of a paroxysm."* "Let me next observe," he says, "that whatever renders the circulation irregular accelerates the accession of a fit, as then, from the increased frequency of the vascular contractions, blood comes to be more rapidly transmitted through the heart, and thus venous blood is poured more frequently into the arterial system; inducing thus, in a shorter time, the train of symptoms which would inevitably have followed, though at a somewhat more remote period."

CCCXXIII. It is difficult to conceive how one so acute and original as Burns should have been so illogical in his reasoning on the preceding phenomena. In the first place, he observes,

* Observations, or View of the most frequent and impor, tant Diseases of the Heart; by Allan Burns. 
" It must be obvious, that sooner or later an accumulation of blood, highly venous, must take place in the arterial system." This is an assertion without the least support. By accumulation, we understand a heaping up, or adding to, a condition which cannot occur in the arterial system, from the intermixture of the venous blood; because it is impossible to destroy, or, in any measure, change the relative proportions of these fluids. The paroxysm he mentions arrests, in the same ratio, the arterial and venous blood, because respiration, on which they both depend, has ceased to act. If the accumulation be granted, how can this be modified, since no alterations can possibly influence the arterial or venous system individually? The incorrectness of this opinion becomes more evident as we proceed. Let us suppose an individual with the Cærulean disease to be perfectly easy ; his respirations about twenty per minute, and his pulse about seventy; and let us still further suppose, that the foramen ovale and the ductus arteriosus are represented by the letters $\mathbf{A}$ and $\mathbf{B}$, and the regular passage between the right ventricle and the lungs by $\mathrm{C}$, we may state that the quantity of blood which $\mathrm{A}$ and $\mathrm{B}$ transmit under these circumstances to be estimated at eight ounces in the minute, and the quantity sent by $\mathrm{C}$ to be oxygenated in the lungs at twelve ounces in the same time. If, by exercise, or other causes, the 
respirations become thirty, and the pulse one hundred per minute, $A$ and $B$ may send twelve ounces in the place of eight ; but $\mathrm{C}$ must also circulate sixteen instead of twelve in the same period.

It must appear manifest, that no accumulation can ever happen in the way in which it has been stated; and, moreover, it is equally clear, that quickened circulation cannot alter the proportions of arterial and venous blood. The paroxysm is occasioned by the determination of blood to the lungs, either from exercise or mental emotions. When such a conformation exists, the delicacy of the adjusted parts is liable, almost every moment, to be disturbed; and when a paroxysm does occur, the sanguiferous system recovers its ordinary power, as stated in CCC.XIX.

CCCXXIV. We now enter upon the consideration of the abdominal and thoracic affections, constituting the second class; and shall briefly show the influence they exert on the organs of respiration. Among the diseases of the abdomen which tend to disorder respiration and its dependent system circulation, are included tympanites, dropsy, enlarged liver, and tumours. Its functional alterations are pregnancy and distention of the stomach. They all possess one common physical condition, viz. that they diminish the capacity of the tharax. The extent of this change, and the variety and character of its effects, will 
be materially modified by the natural conformation of the system, or any of its parts; by the complexity of the disease, the number of organs affected; and, lastly, by the re-action of many causes which we cannot correctly appreciate.

CCCXXV. It is not by any means easy to point out, in a precise manner, the operation of these different causes in inducing palpitation. We observe, in the greater number of instances, that the breathing is short and hurried by gentle exercise, or from slight mental emotion, when the system is subjected to any of these physical conditions; and we are compelled to acknowledge that the circulation of the blood is dependent on respiration, and is also influenced by every change or disorder occurring in the latter. Therefore, it can scarcely be called in question, that in proportion as we render the performance of a function delicate, we augment the liability to derangements in organs whose every action is regulated by the character of that function. It is on this account that palpitation of the heart and syncope are occasional symptoms in pregnancy, and in other cases in which the contents of the abdomen diminish the free descent of the diaphragm.

CCCXXVI. We have at times palpitation of the heart, or a predisposition to it, in many internal diseases, the operation of which cannot be referred to any physical state, acting either on the diaphragm or directly on any organ connected 
with respiration, but must be regarded as affecting the heart by the disorders which have been gradually induced in the distribution of the vital fluid, by the depressing mental anxiety inseparable from disease, and also by the congestion of blood, which always, more or less, accompanies internal chronic affections of a severe kind.

CCCXXVII. The third class, in which we have tumours, and many anomalous diseases that seem to act directly upon the larger vessels of circulation, will not require a minute consideration. It is so obvious, if any part of the circulatory system, indispensable for life, be disordered in its function by tumours diminishing the capacity of important vessels, that the contractions of the heart will be irregular, or predisposed to this condition, as these depend on the quantity, quality, and motion of the fluid transmitted, that I shall not attempt to enforce the argument by a superfluous examination of the subject. 


\section{CHAPTER XIV.}

\section{Syncope.}

Snct. I.-The manner in which the Sanguiferous and. Nervous Systems are acted upon by those causes which produce Syncope.

CCCXXVIII. There are few subjects connected with the science of medicine so truly difficult as the one we propose to investigate. The variety of causes which give rise to the paroxysm of Syncope, the peculiar and invariable phenomena which characterize its origin, progress, and termination, have tended materially to make our knowledge of its proximate cause indefinite or incorrect. The affection frequently occurs in health, or at least at times when we have no evidence of disease. It is a symptom which is found conjoined with the slightest and most severe indisposition; it occasionally follows the ex. hibition of medicines, or the employment of remedial measures; and its precursory stages, or symptoms, are sometimes induced, being regarded as among the most powerful means we possess in counteracting inflammation.

CCCXXIX. The confusion which pervades the writings and opinions of men on the subject 
of Syncope, is chiefly referrible to two causes:Our ignorance of the independent actions of the nervous and sanguiferous systems, and of the reciprocal and necessary influence which unites the one to the other. When passions or external objects have occasioned Syncope, it has generally been considered that the motions of the heart have been stopped or impeded by the direct influence of the brain upon the moving powers of the blood: when such consequences followed depletion or the removal of an hydropic fluid, inequality in the circulation was regarded as the cause. If we had taken into consideration, that the heart is proved to be independent of the direct agency of the brain; and, still further, if we had reflected that the symptoms are invariably the same, whether they be referred to sensation or mechanical alterations,-we should probably have exercised our faculties to discover the intermediate link which apparently connects the mental antecedent to the corporeal sequence in the production of Syncope.

CCCXXX. There is not any other derangement of the body that shows, equally with this affection, the reciprocal influence of the two great, systems of the constitution, viz. the nervous and sanguiferous; and, without having recourse to the flow or suppression of a nervous fluid, and without bringing forward any gratuitous assumptions, it is not difficult to demonstrate the sim- 
plicity of the phenomena with respect to cause and effect, and also the consistency of the general views which have been proposed to explain many other derangements or diseased states of the animal economy.

CCCXXXI. Dr Cullen divides the remote causes of Syncope into two general heads. "The one is of those causes exciting and acting in the brain, or in parts of the body remote from the heart, but acting upon it by the intervention of the brain. The other general head of the remote causes of Syncope, is of those existing in the heart itself, or in parts very immediately connected with it, and thereby acting directly upon it in producing this disease." * The division would be correct, if the facts on which it is supposed to be founded were not, in part, ascertained to be fallacious. He says, "I must assume a proposition, which I suppose to be fully established in physiology. It is this:- that though the muscular fibres of the heart be endowed with a certain degree of inherent power, they are still, for such action is as necessary for the motion of the blood, very constantly dependent upon a nervous power sent into them from the brain." $\dagger$

CCCXXXII. Since the time of CULLEN, the experiments of Legallois, Wilson Philip, and others, have satisfactorily proved, that the action

* Culden's First Lines, miclxxiv.

† Ibid. MCLXXv. 
of the heart remains when the brain and spinal cord are destroyed. If this be acknowledged, the opinion, that the transmission of the nervous power into the muscular fibres of the heart is necessary to excite or sustain its motion, cannot be received in the present state of physiology; and therefore the explanations which he gives of the greater part of the phenomena of Syncope, are theoretical speculations.

CCCXXXIII. In the present chapter, the remote causes of Syncope are divided into two general classes. The first contains those causes which act upon the external senses and the mind: The second, those which act upon the functions of respiration and circulation.

The first class is again subdivided into affections of the senses, such as past impressions, and the pleasurable or painful states of the body.

The second class may be subdivided into those causes which act directly or indirectly upon the heart and the respiratory function.

CCCXXXIV. In investigating the action of the individual causes included under each subdivision, we shall find that every one of these tends to produce the same proximate cause-an overcharged state of the heart. This condition of the heart should not be regarded as an effect of its own debility, as generally believed, but is to be referred to the greater demand made upon its powers by 
every one of the above causes which occasion Syncope. It is observed by a physiologist of considerable talent, that "whatever increases or lessens the vigour of one part must increase or lessen the activity of the whole; though the temperature of the heart itself be no way affected, its irritability is instantly diminished."*

CCCXXXV. The irritability of the heart cannot immediately be influenced by the increased or diminished vigour or activity of one part of the system; its action may be excited or depressed, but this will arise from the changes of its natural stimulus, the blood. If this be highly stimulating, its motions will be accelerated; if deprived of its usual invigorating qualities, its frequent and small pulsations will indicate, that such is the state of the circulating fluid, and not that the irritability of the organ itself is so far lessened as to be the cause of this difference.

CCCXXXVI. It is imagined, when the heart ceases to contract, that it continues quiescent until it has recovered its accustomed energy to enable it to propel its contents. This idea is borrowed from observations connected with other phenomena of the system. Repose to a moderate extent refreshes and strengthens our mental and physical powers ; and from these facts we conclude, that repose will also be attended by similar consequences to the heart.

* Dr Kellie, Edinburgh Medical Journal, Vol. 1. p. 308. 
CCCXXXVII. The muscles of the arm become weak if long exerted, or if put into an unnatural position; but it is not the province of these muscles to dilate and contract upon their contents, in order to propel the blood regularly to other organs; alternate rest and activity are - necessary for their office. The heart is the centre of circulation, and the dependence of every function on its unceasing action would seem to imply, that activity alone is the condition in which it can be invigorated; and it is also found from experience, that almost every cause, whether continued or occasional, which augments its impulse increases its muscular development.

CCCXXXVIII. The heart, in a paroxysm of syncope, is sometimes instantaneously arrested in its motion. When this is the case, we cannot consistently say that it is weakened, because it has not been over excited, nor have we a right to suppose that it is recruiting its nervous energy during the state of quiescence.

CCCXXXIX. The general symptoms which precede and characterize syncope are in the greater number of instances the same, but the extent and variety of these are occasionally much modified by the severity and suddenness of the fit. If the symptoms are gradual in their progress, we have nausea, vertigo, pain in the epigastric region, sensation of cold, tremors of the extremities, paleness of the countenance, lividness and 
trembling of the lips, and cessation or palpitation of the heart. At times the attack is so instantaneous, that the individual presents few of these precursory signs.

CCCXL. The subject of nausea has been studied by almost every physiologist ; but we possess at present no theory concerning it which is deemed satisfactory. The derangement in the circulation of blood in the head has generally been received as constituting the proximate cause. The reasonings which I shall employ, and the facts which I shall adduce in support of them, conjoined with the operation of remedial measures which are found to remove or alleviate the feeling, will probably throw some light upon the subject.

CCCXLI. Nausea, in whatever way it is produced, whether by the recollection of something painful or some immediate affection of the senses, or whether from the undulating motion of a vessel, or the removal of an hydropic fluid, has the same proximate cause-an overcharged state of the lungs.

CCCXLII. In every instance, nausea arises either from a sensation communicated by the brain to the respiratory functions, or from a sensation occasioned by a peculiar state or change of the general system, and which is immediately transmitted to the cerebrum, and afterwards re- 
acts upon the function of respiration, or from the regular course of the circulation being disturbed. The established succession of inspiration and $e x$ piration is disordered when the mind is directly or indirectly concerned in the production of syncope, and it will be seen by subsequent illustrations, that a quantity of blood greater than what is natural is drawn to the chest by the sensations which accompany nausea.

CCCXLIII. After having reflected for some time on the principles which have given rise to the present work, it was gratifying to discover, that the late distinguished philosopher, Dr WoLLASTON, had also remarked a material change in the order of his respirations during the feeling of sea-sickness. Although our ideas concerning the operation of this change by no means coincide, yet it is corroborative to bring forward the views of so able an observer in support of the tendency which the respiratory functions have to promiote the sensations of nausea. The opinions of this philosopher being, on all occasions, valuable, I shall transcribe them. "I had the good fortune to observe a peculiarity in my mode of respiration, evidently connected with the motion of the vessel, but of which, in my then enfeebled state, I was unable to investigate either the cause or consequence. In waking from a state of disturbed sleep, I found that my respirations were not taken with the accustomed uniformity, but 
were interrupted by irregular pauses, with an appearance of watching for some favourable opportunity for making the succeeding effort ; and it seemed as if the action of inspiration were in some manner to be guided by the tendency of the vessel to pitch, with an uneasy motion.

"The sickness occasioned by swinging is evidently from the same cause as sea-sickness.

"It is during the subsidence (of the vessel) that the blood has a tendency to press with unusual force upon the brain."*

CCCXLIV. Dr Wollaston tried to induce the same sensation by swinging; and when this was present, he endeavoured to remove it by making deep inspirations. He says, "Although the advantage was manifest, I must confess it did not fully equal the expectations I had formed from my experience at sea." The result of this. experiment is in accordance with the principles proposed. It is the more frequent or irregular inspirations that are the cause of the various symptoms.

CCCXLV. There is a great difference between an involuntary and a voluntary inspiration. In the former the whole system is relaxed, or predisposed to increase the full effect; in the latter, the very attention or desire of the mind to enforce, counteracts, in part, the consequences we anticipate.

* Lond. Phil. Trans. 1810. 
CCCXLVI. "It is during the subsidence that the blood has a tendency to press with unusual force upon the brain." Dr Wollaston supposes this to be the case, as he feels, at that time, more than at any other, the unpleasant sensation. It is most assuredly during the subsidence that the sensation of nausea is particularly experienced; and any one that has watched individuals at this time must have observed, that there is an appearance not unlike that of fear or dread pictured in the countenance; the raised chest and anxious expression, indicate one continued inspiration.

CCCXLVII. If we can by any means divert the attention of the mind by an interesting story or anecdote, the proper order of inspiration and expiration is maintained, and the nausea is unfelt for the time; but allow the individual to look upon the undulating waves, and to attend to his own sensations, and he instantly becomes considerably worse. If we raise a slight alarm of danger, the fainting, retching, and vomiting almost immediately cease. The natural impulse to preserve life inspires fresh efforts, and these efforts necessarily break the irregular chain of respiration ; expiralions, either more perfect or frequent, succeed, and destroy the order of the inspirations, and, as previously explained, diffuse new vigour throughout the system.

CCCXLVIII. I had an opportunity, a short time ago, of experiencing a slight attack of sickness at sea; and I have also induced it by other 
means, that I might carefully examine its influence and the sensations which accompany it. From observation and experience, and from a knowledge of those measures and remedies which are found beneficial in its treatment, I do not. hesitate to affirm, that there is, in every stage of nausea, an increased quantity of blood in the chest, and a diminution in the head; but whether the symptom be present because the head has lost or the chest acquired, it is difficult to say. At this period the face and extremities have less blood than in the state of health; the temperature of the body is also diminished, because the increased quantity of blood in the chest lessens the capabilities of the lungs to produce. the ordinary effects upon it, in accordance with the principles of the first chapter.

CCCXLIX. Regarding the proximate cause of nausea as depending upon, or accompanied by, an increased quantity of blood in the lungs or thoracic viscera, when suffering from sea-sickness, I took a small portion of brandy, for the purpose of exciting the internal organs, thereby enabling them to regulate the unequal distribution of blood. A few minutes after its exhibition the various symptoms disappeared, and I felt perfectly well ; but, in half an hour, the disagreeable sensation returned; but, by a single repetition of the same stimulus, the healthy conditions of the system were permanently established. 
CCCI. Children are less frequently sick on board a vessel than adults. They are either delighted with the changes and novelties of the scene, or they are too unconscious and unsettled to fix their attention on the varying objects around them, in consequence of which the respiration and circulation remain in their natural state; no disagreeable impression is transmitted to the sensorium, nor is the mind, from their years, capable of that reflexion which readily communicates the influence of mental anxiety to the respiratory functions.

CCCLI. Delicate females are more easily affected at sea than the robust of their sex. Medicines to which we are unaccustomed often promote sickness, although not intended to produce this effect, but, if frequently repeated, they can be taken with comparative ease; and the great class of those agents which never enter the system, but which operate exclusively upon the mind, occasion results precisely similar to those which are the consequences of emetic substances in contact with the mucous membrane of the stomach.

CCCLII. Having divided the remote causes of Syncope into two general classes, I shall say a few words concerning the first, and its subdivisions : that is, into those heads in which we have sensations that are derived from the senses and past impressions, and into pleasurable or painful states of the body. 
CCCLIII. Sensations have two sources, - the external world, and the various conditions of the animal frame. Those belonging to the first are extremely numerous and complex. Education, habits, and associations, influence very much their operation. To some, the odour of flowers, generally admired, is disagreeable; objects that please one offend another. Indeed it is impossible to adduce a single instance in which the external senses, in different individuals, are similarly acted upon by the variety of agents. But if the fragrance of a flower produce syncope in one, it is not because the effect is confined to the sensorium, but because the organ is powerfully influenced, and the impressions it receives are communicated to the respiratory functions; and it is to this re-action that we are to attribute the cessation or palpitation of the heart. The dissimilarity in the sensibility of different individuals, and in the consequences induced, is to be considered as principally originating in structural modifications which we cannot appreciate. If the mind of one individual be more susceptible than another, and if the relations existing between the heart and lungs be extremely delicate, from causes which were stated in the preceding chapter, the mind will be more readily excited or depressed, and every thing within the sphere of its influence will be proportionately disturbed. 
CCCLIV. I have previously endeavoured to show that the heart is not directly affected by the mind through the intervention of nerves, but by its action upon the function of respiration. The muscles of respiration are partly subject to the influence of the will, and partly free from its operation. The involuntary influence is that power which is particularly called into action by our sensations, whatever be their origin or character. If of an inspiratory nature, the ordinary motion of the chest is disordered, and the consequence of this change is, that a quantity of blood greater than what is natural is determined to the thoracic organs, creating palpitation from the increased demand made upon the heart, or complete cessation, from the great oppression.

CCCLV. When fear makes a powerful impression upon the mind, the individual draws a deep sigh, or seems to give a continued inspiration; faintness is probably felt ; and the mode of inspiring being but slightly interrupted by the occasional or imperfect expirations, the lungs and heart receive more blood than they are capable of transmitting with accustomed regularity. It is the relation of quantity to the capacity and energy of the heart that explains in part the definition of Cullen, "Motus cordis imminutus vel aliquandiu quiescens." As the susceptibility, sensations, and organic adaptations are not alike in 
any two individuals, the effects will necessarily be various.

CCCLVI. Intense emotions of pleasure not unfrequently occasion syncope; this arises from the balance of the mental powers being disturbed by causes that have acted almost exclusively upon that part of our constitution appropriated to sensation. The consequence is promoted by whatever is exquisitely painful or agreeable. The effects of these opposite feelings differ but slight$l y$, if at all, in the vividness of their extremes.

CCCLVII. Syncope is not unfrequently produced by heat, but the general application of this power is not so apt to occasion it as when it is partially employed. When the whole frame is influenced at the same moment, either from exercise or any other means, there is an equality even in the altered circulation which prevents the formation of, or rather does not create that peculiar sensation, the reaction of which, upon the respiratory function, is indispensable to give rise to this affection. When one part of the system is individually excited, this almost immediately destroys the harmony of the circulating powers, the derangement of which is precursory to mental and corporeal changes connected with the production of syncope.

CCCLVIII. The general application of cold is rarely or never the cause of syncope, however, not from the reasons which have been assigned 
for the operation of increased temperature, but on account of the diminished sensibility of the nervous system. The blood which is determined internally would probably give rise to syncope or vomiting, but the cold has, at the same time, blunted the susceptibility of the mind to impressions by the quantity of vital fluid transmitted to the internal organs, and by its less stimulating properties.

CCCLIX. Fatigue is familiarly known to predispose to syncope, and the cause of this is obvious ; when the mind or body has been long exerted, debility is experienced. By excessive exercise of every kind the promptitude and perfection of many functions essential to life are enfeebled or constrained; weariness is indicated by sighs, yawnings, and irregular respiration ; a sense of swooning or lightness succeeds; and as these symptoms augment the paroxysm gradually approaches. Chamberet says, "Dans toutes ces circonstances, la faiblesse et la fatigue du cœur, suite de la faiblesse et de la fatigue generales, sont la cause de la suspension de l'action de cet organ et de la syncope, par consequent."* General weakness and debility do not produce weakness and fatigue of the heart in the manner this author imagines. General weakness and debility, particularly if brought on by great muscular ex-

* Dictionnaire des Sciences Medicales, p. 89. Vol. 54. 
ertion, have a tendency to determine the blood to the internal organs. Fxercise, as long as it was continued with vigour, maintained the natural state of the circulation; but the necessary repose consequent on excessive exercise deprives the system of those stimuli that previously sustained the equality of the circulation throughout the body ; and the absence of these, conjoined with the above depressing feelings, speedily produce an overcharged state of the lungs and heart.

SECT. II.-An examination of the causes zohich occasion Syncope by changing the ordinary course or freedom of the circulation.

CCCLX. Structural alterations in the heart, or in parts contiguous to it, are among the most frequent causes of syncope; but having, in the chapter on Palpitation, treated at considerable length of those organic changes which predispose to that affection, it is unnecessary to repeat the same as tending to give rise to the disease we now investigate. The proximate cause of the two diseases is the same, the only difference between them is in the degree and not in the nature of it.

CCCLXI. Syncope is most generally witnessed in the simple operation of bleeding. From the sensibility of the patient, it sometimes pre- 
cedes the abstraction of blood, or occurs during its flow ; it more frequently takes place when the object of the surgeon is nearly accomplished.

CCCLXII. It is believed when an individual faints during the act of bleeding, that it is because the blood has left the heart. I shall attempt to show that it is because the blood has flowed to the heart, and not from it.

CCCLXIII. If the organs of the animal economy were so finely adjusted as the common opinions of mankind'on this subject would lead one to believe, we could scarcely expect that one patient would be able to lose sixty ounces of blood without this loss being succeeded by syncope, when, in another, the loss of two or three ounces is almost instantaneously followed by this affection. It seldom or never happens till a strong impression be received by the sensorium, and subsequently transmitted to the respiratory organs. The surgeon has no difficulty in ascertaining the symptoms which precede its occurrence ; the occasional deep sighs, the irregular, inspiratory character of the respiration, and the paleness or flitting colour of the countenance, are a few of those changes which indicate its approach. I have repeatedly said, that sighs and inspirations determine the blood upon the internal viscera : the $n a$ ture and preponderance of these acts have invaririably this tendency. 
CCCLXIV. Syncope has not any relation to the quantity of blood which is drawn,-its only relation is with the sensations which precede, attend, or follow the operation.

CCCLXV. "On tying the arm," says Dr MAson Good, "a considerable stream of supply is cut off, and ten ounces of blood flow, in perhaps five minutes, into a basin, which would have otherwise flowed into the heart in the same period of time. The volume of blood is hence diminished, and the heart must collapse or contract itself in the same proportion."* If the heart contracted according to the loss of blood, one would think that its contraction or collapse would be very great when it loses six times the quantity mentioned by Dr Mason Good. But the heart is not thus mechanically affected in its action; it may be influenced by the diminution, but it is only so far influenced, in the first place, as to $e x$ cite an unpleasant sensation, the influence of which disorders the respiratory functions, causing sighs and deep inspirations; and it is these acts that have the tendency to bring an unusual quantity of blood to the chest from the surface of the body, the inferior and superior extremities; so that during the precursory stage of the paroxysm, and particularly during the fit itself, the lungs and heart have more, and not less, of the sanguineous fuid.

* The Study of Medicine, Vol. III. p. 506. Edit. Prima. 
CCCLXVI. It is a very wise provision of $\mathrm{Na}$ ture that such a state of things exists. If the heart and lungs had less blood at the time of the paroxysm, death would inevitably be the consequence. Suppose it to be at rest, from great loss of blood, agreeably to the general opinion; can we conceive the possibility of its revival ? Its only stimulus is the blood; it has ceased to act because it was destitute of this principle: then, it is obvious, that it has no other source to supply its necessities, and therefore it must continue in this state of repose. But, suppose it to have been oppressed by a superabundance, in accordance with the principles proposed, and the difficulties vanish. Respiration being suspended, a further accumulation of blood is prevented. The muscular fibres of the heart are in contact with the blood, which more or less stimulates them to contract ; and how trifling soever this contraction may be, it is gradually overcoming the load by which it is oppressed, and the organ is not supplied with a fresh burden, as respiration is inactive, on which the moving powers of the sanguiferous system depend.

CCCLXVII. The position in which a patient soonest revives is that which is best adapted to relieve the lungs and heart. The individual is recommended to be placed upon an inclined plane, with the head a little higher than the trunk. In this way, the blood, however slight the impulse which propels it, has its natural dis- 
position to circulate promoted. The circulation, by degrees, extends itself to the head, extremities, and surface of the body, and it is evident that recovery will be proportionate to the facility with which the distribution to every other part of the system is effected, as it tends to remove the congested condition of the internal viscera and vessels. If the head were placed much lower than the trunk the blood would flow with still greater ease from the chest to the brain ; but this change, instead of relieving the powers of life, would probably destroy them, by preventing the return of sensorial sensibility, indispensable to the recovery of the individual.

CCCLXVIII. In the operation of paracentesis abdominis, syncope very often takes place, particularly if the water be evacuated copiously and suddenly. It is supposed that syncope occurs, because the internal vessels have lost their accustomed pressure. Dr MAson Good observes, when vessels have been pressed upon, as in dropsy of the abdomen, that they " suddenly acquire a power of dilatation." There are several reasons which induce me to dissent from this opinion.

a. Vessels of the head, in Chronic Hydrocephalus, must be considerably more compressed occasionally, than those of the abdomen in Ascites, if either be compressed. The bones of the skull do not so readily accommodate themselves to the power from within, as the parietes of the 
abdomen; it is therefore probable, if pressure affeeted the vessels to the extent which is generally imagined, that the circulation would be entirely obstructed, or, at least, evidently disturbed, which, however, is far from being the case.

b. If internal pressure diminished the calibre of the vessels to the extent supposed, we should certainly expect great disorder in the sanguiferous system, whenever this power was even moderately exerted. We have seldom the enlarged and odematous extremities till general debility be present; and this condition is often remarked when we have no internal pressure to account for it.

c. The only function immediately relieved by the operation of paracentesis abdominis, is that of respiration. It is extremely difficult, or almost impossible, to breathe in a supine posture when the hydropic accumulation is considerable.

CCCLXIX. Whatever alteration the abdominal vessels may suffer from the watery evacuation, I am persuaded that the change, neither directly nor indirectly, is the cause of syncope. During the collection of the fluid the diaphragm has been pushed up, presenting an almost unyielding convex surface to the lungs. The cavity of the thorax, the capacity and mobility of the lungs, have consequently been much lessened and impeded. When the fluid is evacuated, the diaphragm almost instantly resumes its proper 
situation and form; the atmospheric air rushes in to dilate the cells, which had previously been compressed, or almost obliterated ; and the blood likervise, finding less resistance in the chest, flow towards it in greater quantity than usual, producing palpitation of the heart, if the quantity be slight; if inordinate, syncope, from the great oppression.

CCCLXX. The alterations which the abdomen undergoes in parturition may be regarded as operating in the same manner upon the chest and its contents, and we have sometimes the same effects; but Nature, by certain wise provisions, has diminished very much the liability of the female to such occurrences. The first five or six months of gestation are attended by a greater difficulty of breathing and thoracic derangements than the remainder of the period; and this arises from the situation of the foetus, which is principally confined to the upper part of the abdomen, and therefore more injurious to the respiratory function; but, from this time to the period of confinement, it gradually descends, and occupies the lower region of the abdominal cavity, relieving the function of respiration. During the pains of parturition the foetus descends still more, till at last it reaches the pelvis. The gradual changes during labour having the tendency to effect this end, the sudden expulsion of the foetus is not accompanied by serious results; because the respiratory organs have, by 
degrees, in this manner, been protected from effects that would otherwise have destroyed or endangered life.

CCCLXXI. The various stages of gestation, and the act of parturition, are among the best illustrations that can possibly be brought forward of the origin, transmission, and influence of sensations, and of physical changes to which the delicate frame of the female is highly susceptible. 


\section{CHAP. XV.}

\section{Physiology of Passion.}

\section{Sect. I.-The Seat of Passion.}

CCCLXXII. The endeavour to trace the connection between mind and matter has been frequently made; but the inquiries to which it has led have added little to the most ancient opinions concerning Passion. Anatomy and physiology have certainly made us better acquainted with the structure and functions of organs, and with the mode in which these reciprocally act on each other; but, instead of removing the difficulties which envelope the nature of this subject, they have tended to increase them. A superior knowledge of the nervous system has enabled some to form many fanciful theories concerning the operations of mind, such as the oscillation of the nervous fluid, the peculiar action of ganglia, and the direct communication of different nerves.

CCCLXXIII. When an investigation is of trifling moment in its results, we may occasionally allow the ingenious mind to indulge in its visionary speculations; but when the interest of humanity is a prominent object, the flights of imagination ought not to take the place of sound 
reasoning and correct deductions. The speculative physiologist and philosopher more frequently retard than promote the progress of science. The splendour of great names attached to systems or opinions gives these authority and currency, not only when they are well founded, but even when they are gratuitous assumptions.

CCCLXXIV. Since we know nothing of the nature of mind, and almost as little of certain properties belonging to the nervous system, we are by no means prepared to develope principles that necessarily suppose a knowledge of ultimate causes. Although certain secrets of our constitution appear impenetrable, yet it is within the comprehension of human faculties to observe the succession of phenomena, to mark their order and importance, and, by the light which they afford, to distinguish, in a clear and consistent manner, the relation existing between the mind and the vital organs of the body.

CCCLXXV. The labour of tracing consequences to their source is tedious and irksome to the generality of individuals. The progress of science, thus regulated and measured by gradual steps, is necessarily slow, and the object attained appears trivial, unless viewed in connection with others of the same or similar character. It would seem the natural disposition of mankind to systematize, rather than to collect and arrange individual facts by close observation 
and unprejudiced judgment. The eye of taste delights to look upon an edifice complete and beautiful in proportion, possessing a variety of qualities adapted for embellishment or utility, and the mind is similarly constituted : the creations of others, or its own, seldom please unless the plans or principles unfolded are extensive and harmonious in all their parts, a circumstance which seldom occurs, even in appearance, except in those cases in which theory or hypothesis is employed to fill up the interstices or vacuities left by sound reasoning or experimental philosophy.

CCCLXXV1. In the following attempt to investigate the connection between mind and body, I have not been guided by any opinion respecting the nature of the former; it is sufficient for my purpose to ascertain that ideas and sensations are properties of the one, as motion and sensibility are the endowments of the other.

CCCLXXVII. The views which I bring forward are less the production of the closet than the result of close and patient observation on man in the busy theatre of the world, where he is continually agitated by the vicissitudes of fortune or the ever-changing incidents of life. I have attended more particularly to the extremes of mental emotion than to the slighter and more continued influence of less evident feelings, as I propose to reason only from effects ; but what- 
ever laws or principles may be advanced to explain one class of phenomena will apply equally well to another, whose only difference lies in the degree of the cause.

CCCLXXVIII. One part of the philosophical world is disposed to regard mind as an immaterial principle, independent of the direct control of organization ; another would seem to measure its profundity, or estimate its limits, by the quantity of matter presented to the eye. In subjects of this kind it is difficult to make even an approximation to truth, as the phenomena of which we treat are inexplicable on any known law, and are too mysterious to be fathomed by the faculties of man, in a manner sufficiently precise to enable him to refer them to certain well established principles of the mental constitution.

CCCLXXIX. Effect and cause in the animal economy cannot be compared to those arising from ordinary physical adaptations. In the latter we have a clear and distinct idea of the relation existing between the two; but in the former the conception is indefinite and inconclusive, as a variety of causes predispose to produce one great and evident result, the nature and operation of which are not understood : or if one agent be considered the efficient cause, our knowledge even of this will necessarily be confined to its most obvious influence, which will give us no insight whatever into its own nature or 
mode of action; and therefore the simplicity of material arguments becomes complex as soon as it presumes to explain the grounds of its own existence.

CCCLXXX. Passion, by which term I mean every violent perturbation of the mind, has its origin undoubtedly in the brain. Different physiologists have given it different seats; by some it is inagined to reside in the above-mentioned organ,-by others in the ganglia of the sympathetic nerve,-by Bichat, and many distinguished individuals, in the viscera of the body. Passion may be considered a part of the mind, and is as essential for the continuance and regularity of every function of the system as the intellectual qualities themselves.

CCCLXXXI. We are so constituted by nature, that our feelings and mental powers reciprocally act on each other. Reflection, as it unfolds the baseness of those who possessed our confidence, excites our various passions,-and in how many instances do the latter give energy to the desponding exertions of the former !

The intimate connexion between these opposite states of the mind, strengthens the opinion that they both possess the same general organ, or at least one formed of similar materials.

CCCLXXXII. We have not only proofs in support of this supposition in the observations of their ordinary actions, but we arrive almost at 
demonstration in tracing their consequences in an extreme development. At one time our passions destroy the balance of the intellectual faculties, at another these annihilate the proper manifestations of the former; and as insanity is to be attributed to one or both of these conditions of aberration, it is certainly most philosophical to refer every species of this affection to the disturbance of the sensorial functions. The warmest advocates for visceral passions, if I may be allowed the term, would be unwilling to treat mental diseases, even if they could prove the dependence of these on violent perturbation, on those principles which have a particular application to the different organs of the system.

CCCLXXXIII. In individuals who have suffered long from insanity, the brain is frequently found, after death, to exhibit indications of disease; and although we are unable to detect them in all instances, the apparent absence of such is no proof whatever that they do not exist. Before we can decide on the morbid state of any part of the body, we must previously be acquainted with those appearances characteristic of health. This knowledge is seldom accurate or extensive in regard even to those organs whose functions are evident, and whose structure is easy to explain ; but this knowledge, in relation to the various offices belonging to the nervous system, is still more uncertain and superficial. 
CCCLXXXIV. The external form of the head has of late years been much studied in the spirit of experimental philosophy, and is now divided into general and individual sections. General observations go far to support the correctness of the first, viz. the division into those regions appropriated to intellect, sentiments, and feelings. If these principles (in their outline) be received as legitimate conclusions, the seat of the passions becomes as obvious as that of vision.

CCCLXXXV. The opinions and actions of the vulgar have a greater influence in controlling the progress of philosophy than is generally imagined. It is almost impossible, however talented the mind, to investigate causes, and to weigh effects, unbiassed by the many motives and considerations which are borrowed, perhaps unconsciously, from society. The truth of this observation is illustrated in the manner in which Bichat has treated the seat of passions, believing them to belong to the different viscera. To prove the soundness of his views, he could not appeal to experiments, by which he, on other occasions, has distinguished himself, and therefore was confined to reasoning, which is principally founded on the influence of emotions on the action of the heart, and the expressions of individuals under such circumstances.

CCCLXXXVI. In fear, the countenance is pale, the lips tremble, and the heart palpitates; but 
if, from observing the effects of this passion, he is induced to place it in the latter organ, he may as logically refer it to any other part of the frame if excited or depressed. A difference in the degree of the consequences occasioned, is insufficient to authorize a distinction of such into causes and effects, unless in relation to each other, and in reference to the mind as the primary agent.

CCCLXXXVII. Although palpitation of the heart is a concomitant of fear or anger, yet it is not a proof that either of these has its seat in the thorax, nor does the general reference of mankind to the region of palpitation yield any support whatever to this opinion. The learned and the illiterate act equally from the impulse or feeling of the moment, in regard to the external expressions of nature. The hand is frequently placed over the heart when we are agitated by the most tender or elevated passions, whether in solitude or society; and when we wish to communicate to others a knowledge of our sentiments, the same sign is employed. In the first instance, it is almost unconsciously applied to the heart-in the second, because the individual is aware that he speaks most eloquently to his hearers when he brings into action the most significant of conventional symbols; and this is a character as well understood as paleness, or redness, or the expressions of the tongue. 
CCCLXXXVIII. In the early stages of civilization it is not improbable that passion was supposed to reside in the chest, as a peculiar sensation of the heart never failed to accompany the stronger emotions of the mind ; and in those ages most distinguished for refinement and taste the same ideas were entertained, not because men had reflected on the origin of their feelings, and were therefore satisfied of the language in use to express them, but simply because they knew almost as little as those that had preceded them of the functions of animal and organic life.

CCCLXXXIX. The different epithets in constant use to designate a good or bad heart, or the peculiarities of the head, have arisen from the same principle as that which gives a visceral locality to the passions. The greater part of violent emotions being placed in the thorax, and individuals being good or bad according to the development or direction of such, and, still further, mankind being supposed to err from the improper government of such feelings, it became proverbial to say that the heart was virtuous or wicked.

CCCXC. These general observations soon led to others of a more minute description. The heart being regarded as the seat of passion, it was remarked that the most generous and liberal characters were those whose feelings were readily excited by the variety of external objects, and that such individuals had the open, ruddy, and 
kindly expression of countenance, as indicated by the sanguineous temperament, agreeably to their undigested notions concerning the relation existing between the heart and the moral action or sentiments of the mind.

CCCXCI. That physiologists in modern times should have been so far regulated by vulgar opinion as to adopt it in their arguments, in support of the same views, is somewhat extraordinary. If the organs which were endowed with passion had exhibited no obvious functions, it might then have been reasonable to attribute to them the origin of every emotion; but since there is no viscus in the chest thus unoccupied, we cannot allow the imaginative mind to indulge in its fancies at the expence of common sense.

Sect. 11.-The Phenomena and Physiology of Passion.

CCCXCII. Thene are few terms more frequently employed, or more abused, than that of passion. It is common to bestow it on a variety of states of the mind that have not the least similitude to each other, either in the causes by which they are determined, or in the effects by which they are characterized. If it be defined a perturbation of the mind, accompanied by visible alterations in any of the general systems of the body, a great number of mental conditions will 
necessarily be excluded from the catalogue of our passions. If we analyse the constitution of the mind, we shall find it extremely complex, even in regard to those faculties which are more or less distinguished by sensation; but if we closely attend to the expressions and actions of individuals, we shall be enabled to form certain general divisions of the mental states of passion.

CCCXCIII. It is obvious that passions excite or depress, and therefore they possess immediate effects upon the system; and unless we observe these effects, we are not entitled to conclude that the conduct of an individual is influenced by passion. Ambition, pride, and vanity, do not display any of those marks by which we understand a person to be warmly exasperated or deeply grieved; we have neither the florid nor pale countenance, the hurried nor retarded respiration, the pulse bounding, or small and frequent: no indications are present belonging to the system by which we know that such are the characteristics of the mind.

CCCXCIV. From the above and other reasons, passions are divided into those that excite and those that depress, and the different conditions of the mind that cannot be referred to either of these classes are designated mental peculiarities. The consistency of this general division will probably be appreciated when the sentiments concerning each subdivision have been fully stated. 
CCCXCV. The principal passions, which are stimuli to the whole system, are joy, anger, hope, and love; and the chief of those that act as sedatives are fear, wonder, melancholy, grief, and jealousy. Ambition, pride, humility, avarice, and vanity, are included in the class of mental peculiarities. The distinguishing character of the two former is, that they are directly excited by inmediate objects, and the extent of the influence is measured by the obvious changes induced; whereas it is almost impossible, by any external manifestations, to assert the existence of the latter. Our passions are seldom entirely suppressed; they may be controlled: but ambition and pride are dispositions of the mind that are sometimes altogether concealed, and, as they produce no particular consequence upon the system, we cannot estimate precisely their nature or degree. The ambitious man may employ the greater part of his life in endeavouring to exalt himself, without having any of his corporeal functions deranged. Deliberate reflection on the best means likely to promote his interest, or the attainment of his wishes, neither excites nor depresses the organic system; and therefore the principle by which he is actuated ought not to be regarded in the light of passion. The same observations apply to the rest included in the same class.

CCCXCVI. When I speak of passions, I allude to these as depending, equally with men- 
tal peculiarities, on the natural constitution of the mind. Our tastes and susceptibilities are certainly very dissimilar; and although some are disposed to consider such as the consequences of civilized society, or of circumstances, yet it is more consistent with my own experience and reflection to attribute the variety, in degree and character, to a fundamental difference in the conformation of our minds. As this opinion is the one most generally entertained, and as its full development does not belong to the subject in question, it is unnecessary in this place to investigate its merits.

CCCXCVII. In glancing at the exciting passions individually, it is evident that these differ almost as much among themselves as they do from those that belong to the other class. Hope, in its prospective view, is one of the most gentle of excitants : it disburdens the present of its weariness or misery, and embellishes the future with all that imagination can create. There are few clouds which it cannot disperse, and few objects which it cannot brighten; but its influence is so mild that it may somewhat be likened to that of the moon, which diffuses more light than warmth. Yet this passion, although apparently so feeble; is of great importance to the animal economy; it is a constant and benignant stimulus to the mind, and, as the involuntary actions of respiration are regulated by the latter principle, it is the 
indirect means of distributing throughout the system blood of a more invigorating quality.

CCCXCVIII. Joy excites the mind more powerfully than the preceding passion; or, from being more connected with feeling than intellect, impresses its effects more strongly upon the countenance.

CCCXCIX. Anger is the most violent of all mental stimuli, and, as it is characterized by a greater variety of phenomena, it will illustrate more clearly the connection of mind and matter. Of what kind soever be the object that awakens our feelings, its primary action is upon the mind; and when an individual receives an insult, or is vehemently exasperated, we observe the fluency with which he pours forth his sentiments, the hurried state of his respiration, and the muscular exertions employed to enforce the character of his feelings. His volubility of tongue changes the regular order of respiration. Expirations are more numerous and complete than inspirations-the effect of which is, that the blood becomes better. oxygenated, from the quantity within the chest being less than natural. The blood, having undergone more perfect chemical changes, becomes more stimulating to the heart; the pulsations of this organ are more frequent and forcible; the increase of contraction, conjoined with augmented muscular exertion, explain the rapidity of the circulation and the highly florid colour which it 
gives to the face. At this stage of anger, it is not uncommon for the heart to palpitate; and this irregularity arises from the more stimulating nature of the blood. The internal cavities of this organ, and the coronary arteries, receive a more than usual excitation, and therefore the function to which they contribute is influenced accordingly.

CCCC. If the violence of anger be long continued, we remark a different train of symptoms. The countenance is swollen, and of a livid aspect; the respiration becomes deep and laborious, and the individual often applies his hand to the præcordial region, from the pain or oppression which he feels in this situation. Expirations having exceeded inspirations to a painful extent, necessarily prevent the free return of blood from the head and other parts of the body; and this fluid, in a short time, accumulates and occasions that bloated expression to which I have alluded.

CCCCI. I have spoken only of one species of anger. There is another, which is more rare, and has often been fatal, from destroying the action of the heart. It is that in which an individual scarcely utters a word, and in which he exhibits extreme paleness. When an emotion is very intense, it seldom spends itself in idle words; it absorbs too much of the mind to vent its indignation in momentary expressions ; and, instead of being a stimulant it becomes a seda- 
tive to the system. Expirations are not excited as in the preceding instance, but the person affected draws deep inspirations; and these having the tendency to bring the blood towards the chest, they diminish the quantity in the face and surface of the body; and the superabundance in the thorax quickly causes palpitation or syncope, from the action of the heart being oppressed.

CCCCII. I have witnessed one or two well-marked instances of the latter description of anger ; and these accord with the principles proposed to explain them. The curled and trembling lip, the scowling eye, and the fixed features, are observed; and, at the same time, the chest deeply heaves, as if to muster force or suppress an inward feeling. If the cause of the emotion were unknown, and a few of its external traits were absent, the individual would be supposed to suffer from fear : the respiration and circulation are influenced in a similar manner in both cases.

CCCCIII. Having pointed out briefly the mode in which the heart is acted upon by those passions that powerfully excite it, is clear there is not that mysterious connexion between the mind and body, so universally believed. Every functional derangement of the heart, if the effect of passion, is occasioned by a primary disorder in the regularity of the respiratory functions, and is never the consequence of a nervous communication between 
the brain and the nerves which supply this organ. The following objections may, probably, be brought forward against the present view : 1 . That the heart is, at times, instantaneously affected with palpitation. 2. That the order of respiration is frequently deranged without being accompanied by a corresponding alteration in the heart.* In answer to these and similar objections, I may here remark, that there are different degrees of palpitation, agreeably to the intensity of the causes that produce them; and although we speak of palpitation as a general term well understood, yet we must not forget that the recognition of this abnormal action depends as much upon the capability of the mind to attend to its sensations as upon the irregularity in the function of the heart. If we were always equally sensible to every change of this organ, it is not improbable that we should become so familiarized with the variety of its pulsations as to be entirely unsusceptible of its present occasional disorders. The heart is beating more violently when the system is stimulated by spiritous liquors, by running, vehement speaking, and many other kinds of muscular exercise, than in the generality of those cases in which we complain of palpitation; but, under those circumstances, the mind is also excited,

* For the better understanding and appreciating the causes which disturb the thoracic organs, the reader is referred to the Chapters on Palpitation and Syncope. 
and, on this account, is not always sufficiently at rest to analyze feelings of this nature. The nervous patient, whose every moment is devoted to the discovery of new or uncommon pains, regards the palpitation of the heart as the most constant and troublesome of all symptoms; but when we apply the stethoscope to the chest, it is not unusual to find the action as regular as in the state of health.

From these remarks, it is manifest that palpitation, in many instances, may be supposed to exist where there is no increased or irregular action of the heart, but a greater susceptibility of the mind to the ordinary operations of the system.

CCCCIV. Several cases are on record of individuals who were able to arrest the motions of the heart, shewing that these were under the direction of the will. It is impossible to doubt the fact, but the manner may be called in question. From the great power we possess in exciting or depressing respiration, and the direct influence this has upon every state of the heart, it is probable that the action of the organ was stopped by exercising the will upon respiration, and not upon the heart. We are not to infer from this surmise, that every individual is capable of performing the same act, nor do I imagine it possible to be taught; because I believe, whenever this phenomenon occurs, that there is some 
defect or peculiarity in the organization of the heart itself, on which account it is more liable to be deranged by every alteration in the respiratory function. If we suppose its cavities to want their proper proportions, il will be easy to conceive that a quantity of blood, more abundant than usual, will enfeeble or control its action; or some other predisponent condition may exist of which we have no idea.

CCCCV. I have placed Love among the passions of this class, which we have hitherto considered : but it is difficult to say to what division it properly belongs; it varies according to the nature of our feelings and motives, the attractions of the object, the difficulties opposed to our wishes, and many other circumstances not easy to enumerate. This passion is at one time gentle and soothing, - at another it distracts by its violence, or depresses by its fears; but every diversity which it displays may be referred to one of the two general classes.

CCCCVI. From this very brief account of the way in which exciting passions derange the circulatory system, and from what has also been previously stated in the preceding chapters, I hope it has been satisfactorily shewn that the mind has no direct agency upon the heart; and still further, that it is of vital importance to cultivate pleasurable and buoyant feelings in our domestic or friendly circles; not for a momentary 
gratification, but because such are stimuli to that function whose office it is to improve the condition of the blood, and to regulate its distribution,-circumstances so important, that every other function, whether intellectual or organic, operates in extent and correctness, according to the nature and quantity of this fluid.

CCCCVII. The next class of passions we review is that of the depressing. The general observations which I made in speaking of those that excite, concerning the essential qualities of passion, apply with equal force to the present. To illustrate and explain the phenomena of the former, I selected Anger, as presenting extremes in its development, and therefore more likely to be seized by the common observer. To facilitate the accomplishment of the same end, in regard to those that depress, I shall dwell particularly on Fear.

WHYTT, in treating the subject of passion, remarks, - "The paleness from fear may arise from a different cause, (he had previously spoken of paleness from anger,) viz. a deficiency of the nervous power; hence, though the small vessels are not affected with any spasm as in anger, yet they are, in a great measure, deprived of their alternate contractions, to which the motion of the blood in them is principally owing." Instead of bringing forward hypotheses of this kind, in support of which we have not the least evidence, I 
prefer discussing the merits of general changes in the mode of circulation, as such are apparently more consistent or more easy to demonstrate.

CCCCVIII. When an individual is acted upon by fear, the first symptoms we perceive, are deep inspirations and paleness. I look upon the latter as invariably the consequence of altered respiration. The veins of the face have the same dependence upon the action of the respiratory organs as those belonging to the whole surface of the body; and when the influence of the cause applied to the mind is violent, the general capillary system suffers equally; and when this is moderate, the face seems alone to exhibit a sympathy, on account of its vicinity to the heart, and being also the seat of expression. The former circumstance places it more immediately under the control of the disordered function of respiration, and the latter, from the delicacy and sensibility of the part affected, allows us to mark every sudden change.

CCCCIX. Inspirations, as frequently alluded to, have the tendency to bring the blood towards the chest in greater quantity than usual, and, if we have a superabundance here, it is obvious that a diminution will occur in other situations; and it is these modifications in its distribution that explain the paleness of the face, coldness of the extremities, and the oppression of the chest in fear. It is not intended by this opi- 
nion to imply that the lungs are the only organs in a state of venous congestion, or in a condition approaching to it: the abdominal viscera will also be implicated in the disorders induced.

CCCCX. It is common for the heart to palpitate in fear; and, whenever this happens, it is not from the mind acting directly upon this organ; nor is it to be referred to any mysterious agency of the brain : but is to be attributed solely to those changes in the mode of respiration which determine within the chest an undue quantity of blood, and which being greater than the heart can with facility propel, destroys the regular order of contraction and dilatation, as demonstrated by the fluttering or irregular action of the heart. The uneasy sensation will continue as long as the cause remains, and of course will cease in proportion as the sanguineous fluid assumes its normal distribution; which improvement is easily perceived, from the capillary vessels of the face, and other external parts of the body, presenting their usual fulness or colour.

CCCCXI. The coldness which is experienced is not owing to a condition of the nerves arising immediately from the influence of cold, but originates from the deranged circulation of blood; and this feeling is present whenever this state is induced, whether by external or internal means. Intermittent fevers, spasmodic asthma, nauseat- 
ing medicines, and the commencement of most inflammatory diseases, are illustrations of the correctness of the opinion. The same physical alterations may occur without an individual complaining of its influence, or apparently being conscious of its presence, as is frequently observed to be the case when the mind is too much absorbed by an interesting tale or discourse, or when the sensorium does not possess its ordinary sensibility to take cognizance of those impressions which the nerves transmit.

A still further illustration, in support of this view, and of the explanation already given of certain phenomena characteristic of fear, is found in retracing the transactions or amusements of our juvenile years. On a winter evening, when assembled together to listen to the credulous matron, who felt all she described, and whose stories, from their interest, suspended every faculty except attention, and obliterated every feeling save sympathy-the pale countenance, heaving chest, and palpitation of the heart, expressed sufficiently well the depth of our sentiments, and indicated clearly the cause to which the effects were attributable, viz. the functional disorders of respiration. During the continuance of the interest, no shivering or coldness was felt ; but when we approached towards the close, when no further mystery remained to excite or absorb, 
the mind became susceptible of the sensation of cold.

CCCCXII. Persons have frequently died from the effects of anger and fear; but the proximate cause of death in these two passions is as opposite as the feelings by which they are accompanied. In the former, from the expirations being long continued and violent, the return of the blood from the head is impeded, and apoplexy is the consequence; but, in the latter, the head and the surface of the body have less, and the lungs more, of this vital fluid, than in the natural state of the system. The surcharge of the lungs explains the difficulty to which the heart is opposed in circulating the blood, and, when the accumulation is great, accounts also satisfactorily for the complete cessation of the action of this organ.

CCCCXIII. It might probably be expected that we should here treat the subject of Melancholy, as being of a depressing nature; but as this belongs to the character of the temperament, and is rather continuous than temporary in its results, it is evidently excluded from the definition I have given of passion. Cheerfulness has the same relation to the previous class we have considered, that melancholy has to that of the depressing; and it is from the same reasons that it was not mentioned when $I$ glanced at some of the peculiarities of exciting passions. 
CCCCXIV. Wonder, as it produces instantaneous consequences, and these of an obvious description, is with propriety regarded as a passion. When something is suddenly presented to the mind which is new and strange, it creates a series of emotions from the perplexities and doubts appertaining to the object; and the influence of this is perceived in the pale or vacant expression of the countenance, in the palpitation of the heart, or in the disagreeable sensation of the præcordial region. These effects vary little from those characteristic of fear ; and if we minutely observe the manner in which respiration is performed on the present occasion, we shall conclude that the same general derangement of this important function is the sole cause of the different phenomena belonging to both.

CCCCXV. Grief, although the consequence of an immediate impression of an unpleasant kind, and although it is easily traced to its source, from its direct and evident connexion with antecedent circumstances, yet differs widely from almost every other passion. It originates in the same manner, but instead of disappearing after a few minutes, which is frequently the case with the preceding, it continues at times for days, weeks, and months, and during the whole of this period we have more or less some of those external signs which depict the passion and characterize the nature of its primary development. 
We are almost entitled to call this state of the mind at one time an acute and at another a chronic affection; for in those instances in which it does not subside, like the generality of our violent feelings, it presents throughout its duration symptoms so well marked as almost to authorize the employment of a term which supposes the absence of those indications by which it was in the first place particularly distinguished, and yet, at the same time, a state of aberration too evident to be neglected or unobserved.

CCCCXVI. It is impossible to bring forward any passion that more clearly shows the validity of the principles proposed than that of grief. The constant sighing, or deep inspirations, must necessarily tend to pervert the proper distribution of the blood throughout the system; and must also facilitate the accumulation of the same in the lungs. I shall not multiply any further observations on this passion, nor do I think it necessary to examine the phenomena of any other, as they are all referrible to those states that either excite or depress; and although they may otherwise seem to differ, yet the diversities, when investigated, will be found to consist rather in the degree than in the nature of the feeling, understanding by nature the qualities distinctive of each class, and not the fine and ingenious divisions of the metaphysician. 
SEct. III.-The Influence and Effects of Passion.

CCCCXVII. In all ages the mind has been supposed to operate in a mysterious and extensive manner upon the body, not only in the state of health producing acute affections, but also increasing the susceptibility of the constitution to every variety of disease; and the same power has also been equally efficient in retarding or arresting the progress of general and local maladies. I shall not occupy the reader's attention by an enumeration of facts in support of the opinion, for these possess not only universal belief, but individual corroboration, but shall proceed to develope the principles, which are alone sufficient to explain the nature of the connection and the character of the results.

CCCCXVIII. Exciting passions are considerably less dangerous than the depressing to the well-being of the constitution. The former seldom excite or predispose to any disease, except active aneurism of the heart, apoplexy, or congestion of the brain; they very rarely predispose to disorders of the lungs, or tedious nervous affections, nor do they enfeeble the digestive functions, nor increase the liability to contagion,-all of which effects are found to follow depressing states of the mind.

CCCCXIX. The strong and vigorous constitution is almost proof against contagious and 
epidemic diseases, if it have not, for a short time previous to exposure, been deranged by excess of some kind. The reason of this will probably be understood from the following considerations. The blood is the only general stimulus of the body, and the different organs will be excited or depressed according to the properties of this fluid. Whether the agent which produces fever be effluvia from the body, or an impure condition of the atmosphere, it is always to be regarded as a sedative, and its influence is to diminish the powers of life. When an individual possesses superior physical endowments of the system, he is less prone to contagious diseases, because the natural stimulus of the blood is sufficient to counteract the evil properties of that to which it is exposed. It is on this account that persons who are kept in a constant state of excitation from spiritous liquors, can frequently mingle with impunity among those infected with contagious disease ; and it is also from the same cause that fever has in many instances been cut short.

CCCCXX. When I remark that the strong and vigorous are often exempt from contagion, it is necessary to limit the observation, or to state those circumstances which seem on the one hand to support, and on the other to invalidate the opinion. When individuals from the country, possessed of sound constitutional qualifications, are brought within the influence of contagion, 
they are more frequently attacked than others similarly constituted, in the same situations. In agricultural pursuits there are pure air and exercise ; but when those who follow such pursuits reside in, or visit a town, for a period, however short, where fever is raging, they not unusually become affected. We must, in the first place, suppose a specific poison to exist in the atmosphere to which the patient is confined, and to which every person is equally exposed that approaches its limits; but, besides this, there is an unhealthy condition of the air which is breathed, arising from its deficient circulation and from want of cleanliness, to which one unaccustomed to such impurities is more particularly sensible. This state of the atmosphere acts conjointly with the contagious matter; but that is particularly felt by him only who is a stranger to its qualities, and, therefore, it is obvious that he has to contend against this condition of the atmosphere, and also against the common specific principle of the disease.*

CCCCXXI. Professional characters, for the purpose of investigating truth, or relieving the sick, have occasionally devoted their days and nights to attendance on those suffering under the most severe forms of the plague, without falling a sacrifice to its ravages. To him who is fully. acquainted with history, it is superfluous to mention many remarkable instances connected with

* Vide Appendix. 
the influence of the mind on the body. Armies, when flushed with victory, have been able to traverse the most unwholesome situations without detriment to health; and such a feeling has often dissipated malignant fevers and affections, which were previously thinning their ranks. Petix, of Lyons, relates, that during the time this city was besieged, in 1793, every common sore was almost immediately affected with gangrene, and that this consequence was not only observed here, but showed itself in every other place subject to the same unfortu. nate circumstances.

CCCCXXII. In explaining these phenomena, I am forced to recur to certain principles developed at the commencement of this work, viz. that the blood is oxygenated, consequently stimulating, in the inverse ratio of the quantity contained roithin the chest. Depressing passions have one universal tendency, to augment the proportion which the lungs receive, and therefore to deteriorate the condition of the sanguineous fluid. This alteration is not produced in a moment, so as to be sensibly felt by the whole body; it is impossible to fix any precise period for its occurrence, as it is regulated entirely by the susceptibility of the mind, and the severity of the depressing feeling. If we suppose an individual to be continually apprehensive of losing his life, property, or friends, he will sigh, or draw deep inspira, 
tions when reflecting on the probabilities of fortune, and, when not particularly engaged on this subject, his respiratory functions will still show that they are governed occasionally by the im. pressions of the same cause.

CCCCXXIII. Digestion, secretion, circulation, in fine, every animal and organic function, are deranged by the deteriorated qualities of the blood: Whenever this wants its usual stimulus, the constitution will be predisposed to fever ; and if the solids of the body have been for some time supplied with vitiated fluids, those will, on the slightest occasion, exhibit symptoms of mortification.

CCCCXXIV. The above consequences are illustrations of an extreme condition of the body; but if we examine the results of milder causes, we shall observe effects in harmony with the same. If the passion of grief be felt for a few weeks or months, the eye soon loses its brightness, and the countenance its natural expression and colour; digestion is tardy and fastidious; secretion is generally disordered; the circulation is quick and feeble, its distribution is rather $i n$ ternal than external, and the temperature of the body is diminished: but as soon as the mournful object ceases to control the operations of the mind, so soon does this restore to the respiration its normal action, and every part of the system is invigorated with new life.

CCCCXXV. Depressing passions being at 
all times injurious, Providence has so constituted our minds, and the world without us, that it is almost impossible to dwell long on what is disagreeable to our feelings. The variety of our intellectual faculties, sentiments, and passions, is with difficulty concentrated to one point: each having its own individual object, will, as it were, unconsciously steal from the absorbing subject, and apply itself to that which has a much closer relation to its taste; and $\mathrm{Na}$ ture, whether endowed with animal or vegetative life, or whether subject to inorganic laws, teems with those diversities that are well fitted to elevate or expand the mind, or, with eloquence peculiar to herself, will teach us either to overlook or to improve the present.

CCCCXXVI. In those cases in which the mind continues to brood upon its sorrows till the body is too much emaciated to recover its wonted energy, the explanation of the phenomena will be found in the principles developed in different parts of this work. To break the chain of unpleasant associations, the first step is to remove the individual from the situation with which these are connected; or, by lively society and cheerful amusements, to present new objects for thought: or, if he complain of imaginary or slight derangements of the system, to prescribe rather to satisfy his fancy than to cure supposed diseases, The mere exhibition of me, 
dicines forms but a slender part of the professional duties of a physician; and it is to be regretted that his value, in the eyes of a great part of mankind, is estimated by his obvious operations, and not by the obvious beneficial results. There was a time when charms and specifics were held in high repute; and, although we are at present too enlightened to allow the public to be indiscriminately imposed on, yet I hope we are sufficiently candid to acknowledge that these have not only protected thousands from disease, but have saved them when scientific practice had lost its efficacy. Although we are unwilling to imitate servilely the product of ignorance, let us appreciate and apply those principles from which empiricism and priestcraft have derived the greater part of their reputation and consequent success.

CCCCXXVII. Individuals who have suffered long from depressing passions are frequently subject to pulmonary affections. If we consider that the lungs, throughout the mental disturbance, have been more or less oppressed by the congestion of blood, and this of a quality not calculated to stimulate the thoracic and abdominal organs, the occurrence of disorganization ought not to surprise.

CCCCXXVIII. Bichat, in his General Anatomy, relates one or two interesting cases of individuals who had suddenly become jaundiced. 
from the influence of fear ; and others of a similar kind are mentioned by Georget, in his Physiology of the Nervous System ; indeed, examples of this description are by no means rare. Bichat, in reasoning upon the origin of the symptoms, endeavours to show, that fear has an intimate connexion with the liver, agreeably to his notions concerning the seat of passions. He states, that the secretion of bile is increased, as proved by the surface becoming of a yellow colour. The change in the aspect of the body appears to be the only fact from which he deduces his opinion: and as this condition occurs in many instances where such a supposition cannot be allowed, we cannot permit it to pass without examination. Jaundice is common in chronic diseases of the liver, in the obstruction of the biliary ducts by stones, or from any other cause producing the same physical effect. It is often found to take place at the commencement of certain fevers ; and its derangement is evident in many acute and chronic affections of the abdomen: the secretion of bile appears occasionally so much increased, as to characterize particularly one fever.

CCCCXXIX. Whenever the bile is not allowed to take its natural course, it is absorbed and carried along by the circulation through every part of the system, as when the passage is ob. structed by biliary stones or other causes, or by 
tumours in the vicinity of the ducts compressing the parietes of those throngh which the bile flows. In chronic diseases of the liver, this organ is enlarged and congested. In disorders of the abdomen, attended by fever, it is undoubted, that the viscera are generally in a state of congestion; the augmented size of the liver is evident to the hand: depressing passions have the tendency to induce the same conditions. Observing that the influence of fear is to engorge this organ, and, whenever this is the case, that the surface of the body loses its natural colour, I am disposed to infer, that the congestion of the tissue does not promote an increased secretion of bile, but prevents its transmission to its ordinary receptacle, thereby placing the bile under circumstances differing little from those in which its flow is arrested, either partially or generally, by foreign bodies or organic alterations.

In corroboration of this view, it may be remarked, that our treatment in such cases is calculated to disburthen the overloaded vessels of the liver; and in proportion to the success of these means is the disappearance of the peculiar symptoms of jaundice.

CCCCXXX. Diarrhœa is commonly an attendant on fear ; and, as this disease is more frequently the consequence of disordered secretion than an augmentation of a natural action, I attribute it in this instance to the presence of 
deteriorated blood, conjoined with a surcharge of the organs appropriated to secretion. I cannot possibly explain how this quantity or quality of blood acts, nor shall I attempt a solution of the difficulty: At present it is sufficient to show that diarrhoea very often occurs when the sanguineous fluid is determined to the abdominal viscera by the depressing influence of fear ; or, at the commencement of dysentery, the origin of which $I$ have endeavoured to trace in the chapter on the action of Emetics : and as the present investigation includes the same considerations, the reader is referred to that part of the work for a fuller statement of the views.

By these observations it is by no means intended to show that diarrhoa at all times depends on one state of the system, as, for example, the one described; the different conditions of the body that predispose to this effect are numerous, and of very opposite kinds, the elucidation of which does not belong to the subject of passion.

CCCCXXXI. There is another prominent consequence connected with fear, the study of which is equally important as the preceding, and the views brought forward to explain its origin will probably throw considerable light on other phenomena of the system which appear to have no relation to the present subject-I allude to the flow of limpid urine. 
This extraordinary symptom would seem to depend on the same condition of the blood and its distribution that we stated to exist in diarrhœa. In hysteria, nervous diseases, spasmodic asth$\mathrm{ma},{ }^{*}$ and in several febrile affections, the urine is much changed either in its general colour or quantity, or in both. The ancients, who attended much to critical days, considered a favourable crisis at hand when the urine in fever deposited a sediment, or lost its usual watery appearance. The moderns, who observe, are disposed to regard this occurrence in the same light. Why the absence or presence of this simple sign should be believed untoward or propitious has never been explained. Fever never exists without disturbing the whole frame: when ushered in, it generally excites the organic and animal functions, and as long as this action continues the urine is neither particularly copious nor limpid, often in the opposite states : but when the symptoms are changed, and we perceive them to be of an asthenic character, or in any measure approaching to it, the urine is then pale, showing that the blood from rehich it is formed is deficient in its usual properties.

CCCCXXXII. When the internal organs are gradually relieved of the increased quantity and improper quality of the blood, they allow this to

- It has previously been shown that the symptoms which characterise these diseases indicate internal congestion. 
circulate through them with greater facility ; and being of a kind adapted to stimulate the various functions, the change in the secretion of the urine is the result of this general improvement. I have before stated that whenever the circulation is equalized the blood is always better oxygenat$e d$; and this opinion receives additional strength in the examination of these phenomena.

CCCCXXXIII. When digestion is imperfect, we have very frequently a disturbed or muddy state of the urine; and, during the continuance of fever, this and most of the abdominal functions are disordered to a greater extent. The presence of the deposition may, in all cases, save those in which we have disease of the kidneys or bladder, be considered to indicate derange* ment of the stomach or chylopoietic organs. Fever itself is not an exception to this opinion. The copious deposition in the latter stage of fever shows, that the blood and its circulation are improved; the different kinds of imperfectly assimilated matter are taken up by the invigorated action of the absorbents; and, as the urine is derived from the blood, its alterations may be regarded as the result of a better condition and more regular distribution of the vitalfluid.

CCCCXXXIV. Fear has at times instantaneously sobered individuals highly intoxicated and furious. I am acquainted with one remarkable instance of this kind. A gentleman had partaken too freely of the bottle, and was alto- 
gether ungovernable, and, while exercising a few of his combative qualities, he suddenly recollected that he had a considerable sum of money upon him : he instinctively placed his hand where he supposed it to be, and, to his astonishment, found it missing. This unpleasant discovery immediately produced a death-like paleness of the face, and he became as quiet and collected as one that had taken no spirits. After making inquiry concerning it, he learnt that his brother had taken it from him for the purpose of securing it: being assured of the truth of this, he immediately became as intoxicated and ungovernable as before.

Fear, as I have before stated, diminishes the quantity, and probably the action, of blood in the superior and inferior extremities, and on the surface of the body. This change was suddenly induced in the above example, by the supposed loss of the money; and as soon as the alarm was found to be false, so soon did the respiratory function resume its previous action, and reestablish the abnormal circulation of the brain. 


\section{CHAP. XVI.}

An Inquiry into the Nature of the Vital Principle.

Sect. I.-An Investigation of the different Phenomena attributed to an Unknoron or Immaterial Principle.

CCCCXXXV. In undertaking the present subject, I am not insensible that I tread upon ground deemed sacred by the vulgar and the greater part of the enlightened of mankind. The prejudices of youth and of education, and the various associations connected with what is great or wonderful in Nature, tend to retard materially our knowledge of phenomena which are properly within the comprehension of our faculties. The child regards, with infinite pleasure, the different motions of the automaton, or the various actions of the puppet, without being able to consider the cause of its admiration; and the mind of man is also liable to be unduly excited, or improperly directed, in observing the phenomena of animal and vegetable nature, as these differ so widely from every effect characteristic of inert matter. 
CCCCXXXVI. Some have supposed life to be synonymous with soul, and, therefore, assert that it is an immaterial principle, altogether beyond the province of intellectual pursuits ; but others, with apparently more correctness, are inclined to regard life as produced and regulated by the laws which the Deity has impressed on the particles of matter. The individual who entertains the latter opinion has sentiments as pure and elevated as he who refers every act of existence to the direct agency of Providence; nor does the doctrine tend to lessen his admiration of what is everywhere great and good throughout nature, nor does it in the least tend to diminish his reverence and the grateful feelings of his heart towards the great Author of his being. Of the two opinions, that which is deemed material is better calculated to excite wonder and religious awe in one highly gifted, as it presents to the expanded faculties of the mind the infinite laws of his creation,-whereas, when these are referred to the direct agency of Providence, they cannot be considered as a source of wonder, but may of adoration, as the Spirit of the Most High is all sufficient.

CCCCXXXVII. If the general properties of life be allowed to be sufficient to demonstrate an immaterial principle or soul, every link of animal and vegetable nature will be granted 
to possess it; and it still further must be admitted, that this principle is subject to decay and reproduction, to derangement or disease, as its manifestations are deranged or diseased by a variety of causes. The soul and its ultimate destination are objects for the study of the ethical philosopher; but their development and foundation cannot be traced and proved by the bounded powers of the mind,-it is to Revelation alone that we must appeal for a knowledge of these great truths.

CCCCXXXVIII. In examining the qualities of matter completely destitute of the principle of life, we have much to admire. Certain particles combine, and the product is unlike the previous properties of either; poisons become harmless or beneficial by the addition of a new principle, or different proportions of the same of which they are composed; salts and crystals have their determinate forms, and it scarcely can be considered less strange, that such substances should exhibit a definite external character, than that arteries and veins should be developed with the same unerring certainty in the animal system.

CCCCXXXIX. It is as difficult to show in what manner, or by what exact laws, crystals have an invariable tendency to assume the varieties of determinate forms, as to explain in what way an artery or vein is organized. The former phe: 
nomena appear as wonderful as the latter. Both objects are material, but composed of different principles; but whether the difference consist in the simple or compound nature of these, I shall not presume to say.

CCCCXL. Why the particles of inert matter are attracted to each other, and form a nucleus, is unknown; and we are equally ignorant of the cause that induces particles derived from animal or vegetable matter to combine in order to develope the various parts of either of these great systems of nature. Blood-vessels are observed in the foetal germ, before any other organized structure, and, when these are but partially formed, they are prepared to promote still further the objects which nature has in view, as they convey and distribute the maternal blood, which is the exclusive source of every subsequent development. As these vessels become more manifest, the progress of organization proceeds, not precisely as an effect of the first exciting impulse, but as an immediate consequence of the progressive evolution of additional structures, and of the more copious supply of maternal blood; and these causes augment and continue to operate, till the different organs are constituted, which are necessary to maintain an independent organic and animal life.

CCCCXLI. It is extremely difficult to define life. If it be supposed to have any indis- 
pensable relation to the faculties of the mind, to the sensibility of the frame, or the senses with which an individual is endowed, we have vegetation, on the one hand, presenting occasionally the exuberance of life, and, on the other, we have animals almost completely destitute of the above conditions; even man himself exhibits different degrees of perfection in the instruments by which he acquires his knowledge and elevates his natural pre-eminence. We have every appearance of life, where we have neither voluntary motion, sensibility, intellect, nor any of the five senses, and yet we observe that these striking characteristics of life, and the different organic functions, bear a direct ratio to the development and perfection of those organs on which these endowments depend. From such facts, we may infer that life is extended or contracted according to the number or magnitude of the various organs of the system; and, still further, that it possesses its degrees in the same individual at different periods of existence, or in different states of the constitution. The youth, buoyant with high spirits, ambitious from the vigour of his mind, and restless from the energy of his animal and organic functions, must be allowed to possess a greater share of the principle of life than the aged and emaciated being, almost paralysed by debility, imbecile in mind, from his im- 
poverished or ill-conditioned brain, indifferent to the pleasures of society, the joys of sense, and almost every tie that binds the soul to earth.

CCCCXLII. Dead animal or vegetable substances cannot in the least be likened to brute or inert matter. The former contain the remains of those principles that were deemed vital in their operation and effects, viz. the circulating Auids, which are proper to every part of the organized system. As these principles, a few seconds before death, were capable of maintaining the various functions of the living economy, it can scarcely be denied that immediately after this event the properties of the fluids are unchanged. This argument is, in some measure, illustrated in the recovery of individuals from drowning, in transfusion, or certain accidents. Dead animal or vegetable substances, although inorganic, are not destitute of those material principles which supported the complex functions of animal or vegetable life; and these, therefore, when free from organic laws, quickly undergo what is called decomposition, which is owing to the formation of other combinations, and, in consequence of these changes, life appears again under a variety of aspects.

CCCCXLIII. The various organs of the body possess different degrees of vitality in proportion to the quantity of the circulating fluid they receive; and may also be considered vital accord- 
ing as they contribute to the support of the whole system. The lungs, the heart, and the blood-vessels exhibit these two conditions to a greater extent than any other of the constitu. tion.

CCCCXLIV. Physiologists are continually dilating on the wonderful properties of the vital principle, in resisting great heat or cold; in exerting itself, on ex traordinary occasions, as if endowed with consciousness, or fully aware of the necessities which are present. But these individuals do not state, nor even endeavour to explain its nature, while they at the same time entirely neglect to consider the physical conditions of the system, whose changes will be found, from the following observations, amply sufficient to solve many of the seeming difficulties connected with the subject.

CCCCXLV. Many experiments were performed by Hunter which tended, according to his reasoning, to prove, that the phenomena he observed were to be attributed to a vital principle, exempt from the conditions of matter; and the same experiments are brought forward by other writers to enforce the same opinion.

A dead penis, and one of a living person, both of the temperature $92^{\circ}$, were immersed in the same vessel with water at $50^{\circ}$.* He remarked,

* Observations on certain parts of the Animal Economy. By John Hunter, p. 108. 
" that the dead cooled sooner by two or three minutes; the living sunk the quicksilver to $58^{\circ}$, and the dead to $50^{\circ}$." There is nothing extraordinary in this result, but, on the contrary, it is such as we should naturally expect. The dead penis is to be considered as an inorganic substance, deriving its heat entirely from the medium in which it had previously been placed, whereas the living one has its temperature continually renerved by the circulation of fresh portions of blood. The portion which is this moment cooled is necessarily transmitted by the veins to the heart, and arterial blood of the temperature of the body flows hither, and this again is cooled, and these series of interchanges unceasingly operate with different degrees of facility and perfection. When the living penis has been immersed for some time in water as low as $50^{\circ}$, the blood-vessels are constricted, preventing the transmission of the usual quantity of sanguineous fluid, and as that which is acted upon returns with difficulty, the living penis has its temperature very much reduced. The dead penis, deprived of the physical conditions of the living, has its temperature speedily diminished, and necessarily to a greater extent than the other.

CCCCXLVI. In the next experiment, the living penis, having the temperature of $92^{\circ}$, was immersed for two minutes in water, heated to 
113. The heat of the parts was raised to $100^{1}{ }^{\circ}$, but could not be raised higher.* In another experiment " the living and dead parts were both immersed in water, gradually made warmer and warmer, from $100^{\circ}$ to $118^{\circ}$, and continued in that heat for some minutes; the dead parts raised the thermometer to $114^{\circ}$, while the living raised it no higher than $1021^{\circ}$.

When the living parts were immersed in water fourteen or fifteen degrees above the temperature of the system, the heat which they, or more correctly the blood, acquired, was quickly. carried into the system by the veins. The arterial fluid subjected to this warm medium continually acquires additional heat, but this is immediately transmitted by the veins; and it is on this account, and not from the existence of a peculiar vital principle, that the living parts cannot be heated beyond a few degrees.

When cold was employed in the first experiment the capillaries of the penis were powerfully affected, because the return and transmission of the blood rere impeded; but, in the present case, the warm water facilitates both the return and transmission of the blood, and therefore tends to preserve the living parts from an inordinate rise of temperature. There is a circumstance connected with the present experiment, which beautifully

$$
\text { * Ibid. p. } 109 . \quad+\text { Ibid. p. } 110 .
$$

A a 2 
illustrates the correctness of the explanation I have given. When the thermometer was put close to the living penis, after it had been immersed for some minutes in the warm water, " the quicksilver immediately fell from $118^{\circ}$ to $104^{\circ}$, while it did not fall more than a degree put close to the dead." *

The individual who submitted to this experiment did not feel the water unpleasantly warm when the parts had been immersed in it for a few minutes; but whenever the water was agitated it was felt disagreeably hot. It is manifest that the circulation of blood through the penis continually robbed the water in contact with it of a portion of its heat. As the blood was warmed at the expense of the surrounding medium, and as it was renewed every few seconds, the

- water would necessarily lose several degrees of heat. When the water was agitated, another portion, which had not been particularly acted upon by the blood, was then placed in contact with the living parts, which satisfactorily accounts for the heat which was experienced from this simple circumstance.

CCCCXLVII. Richerand, the distinguished French physiologist, has endeavoured to support views similar to those of Hunter. He says, "I applied bags, filled with very hot sand, along the leg and foot of a man whose artery

* Ibid, p. 110. 
had been tied by two ligatures in the hollow of the ham, for popliteal aneurism. Not only the limb was not chilled, which is what happens when the course of the blood is intercepted, but the extremity, thus covered, acquired a heat much above the ordinary temperature of the body. The same apparatus, applied to the sound leg, did not produce this excess of heat, certainly, because the fulness of life in that limb resisted the physical action."*

The explanation of this phenomenon is as simple and satisfactory as the preceding in reference to the experiments of Hunter. An artery had been tied in the leg, which would prevent the limb from receiving its ordinary quantity of blood, and, consequently, the extremity would become less vascular, and the circulation would likewise be irregular. On the application of the sand bags, the sanguineous fluid would be warmed, but the limb not possessing the usual quantity, would, therefore, be incapable of carrying off the external heat to the same extent, and with the same facility, as the greater quantity of bload and its more regular circulation in the sound limb. The extremity in which the arteries were tied, from the great diminution which it suffers in the proportion of the circulating fluid usually

* Elements of Physiology, p. 45. Edit. quarto. Translated by G.J. M. De LYs, M. D ; with Notes and a copious Appendix by James Copland, M. D. 
distributed to it, may be considered as approaching to inorganic matter, and the effects which it exhibits approximate in character to those observed in substances altogether destitute of life when subjected to the same circumstances.

CCCCXLVIII. It is not unusual to observe good, and almost immediate effects, to succeed the amputation of a limb. Paleness, emaciation, debility, and irregularity of the various organic functions, are removed or lessened by such an operation, and these beneficial results are principally to be attributed to two causes, viz. relief from mental perturbation, and a more copious supply of blood to the remaining parts of the system. On the first point it is unnecessary to insist, as the influence of the mind on the corporeal functions is too well known to require further comment. Previous to the amputation of a limb, the chief artery or arteries are compressed, which circumstance prevents the ordinary distribution of the vital fluid to the parts which are to be removed, so that the amputated extremity contains much less blood than is possessed by that which is sound : it is therefore evident that the system has received an increase; and this quantity, although small, may be considered in the light of a natural transfusion, and tends, like that which is occasionally employed in cases of great loss of blood, to revive the sinking powers of life. 
In proof of the great increase of sanguineous fluid to the system, I may observe that thoracic inflammations sometimes succeed the operation, or great irregularity in the functions of those organs necessary to oxygenate and circulate the blood, and the different symptoms are generally relieved by depletion. I have already remarked, in the chapter on Torpidity, CLXXXVI, that, by circumscribing the circle of circulation, we enable the heart to act with greater ease; but, in the instances which I brought forward, we had a diminished quantity of blood in motion ; but when we lessen the circle by the removal of a limb, we throw more blood of a stimulating kind upon the internal cavities, thereby exciting the heart to augmented contractions, the energy of whose effects are immediately transmitted to the lungs and the whole vascular system. These conditions act and react, improving digestion and assimilation, in fine, bestowing new life on the impoverished functions of the body. In such instances it cannot be denied that the relation between the cause and effect is obvious, and the conclusion which naturally flows from such premises is, that the principle of life was weak or diminished when the system was deprived of the necessiry quantity or quality of blood, and that this principle became strong or increased when one or both of these pro- 
perties were present. I shall attempt, in the following pages, to show in what way the quality of the blood is improved, and also what is the agent; we shall then be still better able to judge of the principle of life. These observations suggest to the surgical operator the necessity of preserving, as much as possible, the vital fluid for the benefit of the system, when such is imperatively demanded. This object is accomplished by applying the tourniquet, or similar means, to the trunks of the principal arteries belonging to the limb, for a few minutes previous to the operation. When the extremity is thus circumstaneed, the arterial blood is necessarily very much impeded in its flow to the limb, if not almost suppressed, while, at the same time, the venous does not experience an equal difficulty in its return.

CCCCXLIX. The observations and precepts of mankind, in general, are frequently more valuable than the man of science is disposed to allow. The basis of the greater part of our knowledge has been formed by contributions derived from vulgar opinion and experience; and, even at the present moment, the medical practitioner is, to a very great extent, directed in the treatment of diseases, by rules, if not entirely empirical, partaking considerably of this character. In illustration of these sentiments, it is generally stated 
that children are rendered sickly, or at least do not thrive so well, by sleeping with individuals far advanced in years, and, still further, that the life of the latter is prolonged by such means. From the following observations, it will appear probable that the benefit which the one derives will be at the expense of the other. The temperature of the child is superior, and much more equally diffused, and the circulation is also much more vigorous than the same in an old and weakly individual. In thelatter the extremities arekept warm with difficulty, the surface is pale and collapsed, and the susceptibility to cold at this period of life proves, that the source of animal heat possesses neither great energy nor stability. When the system, in this condition, is placed in juxtaposition with one that developes the strong powers of life, it necessarily deprives it of a portion of its heat, on account of its conducting qualities. The additional stimulus acquired is grateful to the sensations of the aged, and as it tends to excite the external capillary circulation, it communicates a genial warmth and new vigour throughout the constitution, and consequently improves the various organic functions, whose office it is to oxygenate and circulate the blood, or otherwise contribute towards the development of the principles of life. From this explanation, we perceive that existence may be 
prolonged when it is ebbing from the natural decay of the organs essential to its perfection or continuance; but we are not aware that the child possesses a superabundance of animal heat or strength of constitution; and therefore, it is highly probable, when it is thus circumstanced, that its health may be impaired, and the evolution of its future powers may be materially circumscribed.

CCCCL. I have already remarked that life has its degrees in the same individual at different periods of life, and in different states of the system ; and it has also its degrees in various parts of the animal frame. The extremities, from the effects of wounds, ligatures, or exposure to great cold, are more liable to mortify than any other parts of the body. As they are the farthest removed from the source of circulation and animal heat, the vigour of the one and the extent of the other are considerably modified before they reach these distant parts ; and it is from this cause that cold, for example, occasionally produces immediately fatal effects. In this instance, the limb or the toes die for want of the proper quantity of blood; consequently thisfluid contains the principles of life.

The above reasoning explains an experiment of HunTER, which is otherwise accounted for by him. He reduced the temperature of the 
mouth from $97^{\circ}$ to $77^{\circ}$, by introducing small pieces of ice, and allowing them to melt there; and he says that the effect would have been much greater, if the hand, instead of the mouth, had been thus circumstanced. The mouth, from its vicinity to the heart, and from the great quantity of blood which it receives, is cooled with comparative difficulty, and it speedily regains its ordinary temperature from the same cause; but the hand, from conditions precisely opposite to these, is greatly and quickly affected by the same means.

CCCCLI. After surgical operations, or injuries of the body, the separated surfaces very frequently unite by what is called the first intention. This desirable object is sometimes rendered tardy, or impossible, by an impoverished state of the system, or by the gloomy passions of the mind. It is facilitated by whatever excites agreeable feelings, or tends to invigorate the powers of the system. I have already endeavoured to prove, in various parts of this work, that the blood, during the influence of depressing passions, is deficient in its usual qualities, because the sanguineous fluid, by the exercise of such mental states, is brought to the lungs in greater quantity than natural, perverting the conditions indispensable to oxygenate completely the blood, as stated in the first chapter. 
CCCCLII. It is stated by Richerand and others, that " the increase of vital energy to an injured part" indicates "the existence of a preserving principle, incessantly watching over the harmony of the functions." *

This idea seems very correct and beautiful when superficially examined, or rather when not examined at all ; but if we take into consideration, that he here alludes to the blood flowing to a part which is wounded by the prick of a thorn, it is not difficult to bring forward a thousand instances where such a flowing was the cause of great inflammation, suppuration, and death. Indeed, when such cases occur, it is not our object to increase, but to diminish the determination; and if the vital principle were incessantly watching over the harmony of the functions, how does it happen that it destroys its own existence by allowing congestions or inflammations to take place? It may probably be asserted, that it is too weak to resist the influence of powerful agents ; therefore it must be allowed to be serviceable only when its contributions are not particularly required.

CCCCLIII. In the preceding pages I have alluded to and explained many states of the system that have been regarded by the most distinguished physiologists as inexplicable, ex-

* Trans. ut supra, p. 48. 
cept on the supposed existence of a peculiar vital principle, whose attributes are like those of a spirit possessing an unembodied form, sensation, and perception, which is continually on the look out, in order to protect the body from insidious attacks or dangerous breaches, but, when it finds itself unequal to the contest, retires and allows the assailant to devastate the domains it so lately governed and beautified. Having proceeded thus far in the investigation of the nature of the vital principle, I shall endeavour, in the next section, to make still closer approximations to a knowledge of that vivifying principle, by means of which the organic functions and the animal (of course considering the immortal mind as a separate existence) are maintained in the exercise of their various powers.

SECT. II.-An Inquiry into the Nature of that Principle by means of zohich the System is maintained in Health and Vigour.

CCCCLIV. That the principle of life is subject to an increase and diminution, must appear probable to the most superficial observer of $\mathrm{Na}$ ture ; but, if he investigate the subject profoundly, his doubts and probabilities will perhaps be exchanged for certainties. In marking the exu- 
berance of health displayed by the child in the activity of organic and animal functions, he will not hesitate to allow, that this apparent superabundance of powers is bestowed in order to develope or invigorate the various organs of the system. These powers are evidently much greater than would be required to maintain the infantile constitution, if this were intended to be stationary. It therefore ought to be our object to ascertain in what consists the superiority of the child over the adult in regard to those functions that coexist with, or produce the most important changes of the body.

CCCCLV. To arrive at a correct knowledge of the animal frame we possess only two modesdirect experiment, where such is possible, or a close and patient examination of cause and effect. The latter means are very frequently as conclusive as the former. In following such a plan, we are liable to one general source of error, viz. that of seizing only the apparent cause, without being sufficiently aware that another, or others, may intervene between it and the obvious sequence, which alone can be regarded in strict relation with the effect.

CCCCLVI. When the child exhibits that necessary and continued activity of body and mind, we observe the respirations frequent, the contractions of the heart and arterial system much more numerous than in after life; and 
we also remark, that the surface of the body is highly vascular and generally florid at this period. If we turn to the system in decline from age, we perceive that the frequency and vigour of respiration and circulation are strikingly diminished, and, still further, that the restless desire of action, the rotundity of the muscles, and the vascular and florid condition of the surface of the body, no longer exist.

It can scarcely be denied, that if we lessen the frequency or perfection of respiration, we shall deteriorate the powers of life. The excited and generally diffused circulation, in childhood, is a consequence of the stimulating properties of the blood and other conditions, fully stated in Chapter IV.; and, therefore, this state of the circulating system, and every organic function dependent on its influence, are to be referred to the frequency or perfection of respiralion, as the primary source of every effect.

CCCCLVII. We may next inquire how the frequency of respiration can operate so as to occasion these marked results. In the nine general laws, or deductions following XXVIII. I have alluded to particular states of the respiration, and have endeavoured to show that certain of these are the cause of the buffy or inflammatory coat in blood, arising from its highly oxygenated qualities; and, moreover, when opposite respiratory or sanguiferous conditions are present, 
the blood, in harmony with such changes, does not exhibit the same character, and, in many instances, will not even coagulate. The conditions which produce the inflammatory coat in disease, are the same as those that predominate in childhood simply modified. When they exist in disease, we have increase of temperature. augmented action of the heart and arterial system, a vascular or florid appearance of the surface of the body; and when they occur at an early period of life, we have similar symptoms, differing only in degree. The frequent respirations, supposing other conditions of the system favourable, as they are almost universally in childhood, must be allowed to deteriorate, in a given time, a greater quantity of air than when these are slow or imperfect. The air which is inspired contains a vivifying principle, viz. oxygen ; and if a greater quantity of air be breathed in a limited period, a greater quantity of oxygen must be received by the blood in the lungs in the same time; and it is to this agent we must refer the oxygenated or arterialized properties of the sanguineous fluid.

CCCCLVIII. In the child the arterial blood is more florid than the same in advanced life, or at least the venous is not so dark as at this period. When the body is subjected to great heat or exercise, the venous loses its ordi- 
nary well-marked characters ;* indeed the distinction, in regard to colour, between arterial and venous blood, is almost lost. The cause of these phenomena appears so evident, and so closely connected with the effect, that it is almost impossible to refer the latter to any other agent than oxygen. If this be granted, we must acknowledge that the principles or powers of life are to be attributed to the influence of this agent.

CCCCLIX. The blood possessing under various circumstances a greater or less proportion of the vital principle, as indicated by the activity of organic and animal functions, and also by its external qualities, it necessarily follows, if it be highly oxygenated, and characterized by a variety of effects in the living system, that it ought also, when removed, to be distinguished from that which is in a different condition, if both be placed in precisely the same situation. I have already observed that the circulating fluid in the child possesses a greater portion of vitality than the same in one advanced in years; and the correctness of this opinion is demonstrated by the following experiments of Hunter, instituted, not for the purpose of proving the present point, but in order to ascertain the difference in the properties of the blood at different ages.

Vide Chap. VIII.

B b 
He says, "To see whether the blood in a young person or an old one becomes soonest putrid, I desired that the following trials should be made.

"June 24. Some blood was taken from a woman twenty years of age, and its surface, after coagulation, was covered with an inflammatory crust.

" On the same day, some blood was taken from a woman aged sixty, when crassamentum was also covered with an inflammatory crust.

"These quantities of blood were set by.

"The blood from the old woman putrefied in two days; that from the young woman kept quite sweet till the fifth day, when it began to smell disagreeably; in this state it continued two days more, and then emitted the common odour of putrid blood."

"Several experiments were made in the course of the summer of a similar nature with the last, in all which it appeared that the blood from young people kept longer sweet than that which was taken from the old."

It must be acknowledged that the result of this experiment is confirmatory of the principles laid down. Hunter endeavoured to show that the blood was vital, but he did not attempt to investigate the nature of this property. I have stated in CCCCLVI. and CCCCLVII. that the

* Hunter on Inflammation, Vol I. p. 167. Octavo Edit, 
difference between the inflammatory state of the blood in disease and childhood is merely in degree. This opinion is almost as directly proved as the preceding, regarding the condition of the sanguineous fluid at different ages, by the following experiment of the same physiologist: "I wished," he says, " to see if blood that coagulated with an inflammatory crust putrefied later than that which coagulated without it; for I conceived that the strength of coagulation was something similar to the strength of contraction in a muscle, resisting putrefaction. For this purpose I ordered the following experiments to be made.

\section{“ EXPERIMENT I.}

"Four ounces of blood were taken from the arm, which, after coagulation, had the inflammatory crust upon its surface, and was also cupped."

\section{“ EXPERIMENT II.}

"On the same day four ounces of blood were taken from another person's arm, which on coagulating shewed no inflammatory crust on its surface. Both these quantities of blood were kept, in order to see which would resist putrefaction longest.

" By the fourth day, that without buff was putrefied; but the blood with the inflammatory crust did not putrefy till the seventh day." *

- Ibid. p. 166.

B b 2 
CCCCLX. In the preceding chapter on the Physiology of Passion I have endeavoured to explain the influence of those feelings which are of a depressing nature, and, therefore, on the present occasion, shall allude only to such as are in strict relation with the present investigation. It is well known that the depressing feelings of the mind disturb, according to their severity, the regularity of the vital functions. If only slight, the appetite is lessened or becomes fastidious, the ordinary complexion of the countenance is changed, and the energy of the mental faculties is rendered of little avail ; but if such be severe, we scarcely observe the slow and progressive diminution of the powers of life; the individual affected sometimes dies after a few days indisposition; and in such cases it is remarked, that the blood coagulates with difficulty, or sometimes not at all. It is impossible to bring forward a more direct instance in illustration of the influence of the mind upon the body, or one that applies so immediately to the subject of vitality.

CCCCLXI. To those who are well acquainted with the language of grief it is unnecessary to insist particularly on the character of respiration. When an individual is suffering from an affection of this kind, his habits are retired; he finds little or nothing to interest him in the social circle, or in pursuing his ordinary amuse- 
ments or labours : if not excited by conversation, he remains silent, fixed in thought, and continually draws profound inspirations; indeed, we perceive occasionally a series of these acts, in his frequent and deep sighs. I have repeatedly stated that inspirations have the tendency to bring a quantity of blood greater than what is natural to the chest, and the truth of this opinion is exemplified in the present case. I have also observed in the first chapter, that, whenever the lungs possess a quantity of blood greater than what is natural, the chemical changes are materially diminished. The influence of grief prevents the conditions essential to a perfect oxygenation of the blood, as it brings to the lungs an inordinate quantity of it, and, therefore, increases on the one hand the blood that has to undergo chemical changes, without augmenting on the other, to the same extent, the proportion of air within the lungs to produce these effects.

CCCCLXII. From this explanation, it is clear that the properties of the blood are deteriorated, on account of the deficient chemical changes in the lungs, or, in other words, that it is defective in the principle of vitality, from the deficiency of axygen. While a depressing emotion, like that of grief, continues to occupy, or rather absorb the attention of the mind, the blood is gradually losing its stimulating qualities; the surface of 
the body becomes pale; the organic and animal functions are feeble or irregular; the individual frequently complains of oppression in the epigastric region, from the accumulation of blood. The heart, in such cases, is often subject to palpitation, the pulse at the wrist is remarked to be very weak and small, and generally quick; and when these symptoms are well marked, the temperature of the body, in accordance with the character of the above derangements, is less than in the natural state of the system.

CCCCLXIII. It would be easy to enlarge on the preceding remarks, but I can scarcely point out in a more perspicuous manner than $\mathbf{I}$ have endeavoured to explain, the way in which the blood has its vitality diminished for want of the usual chemical changes in the lungs. Observations of this description are important, as tending to explain the way in which the vital fluid is deteriorated; and if the present views be allowed to be correct, we can now, with perfect ease, speak of mental influence, not as a mysterious or immaterial power pervading the whole system, but as a cause which gives rise to the different phenomena, from having disturbed the regularity of the respiratory functions. The principles which enable us to explain these phenomena, by examining the strict relations existing between cause and effect, are precisely the same 
as those by which I have, throughout this work, attempted to solve many difficulties connected with organic and animal life.

CCCCLXIV. In individuals of the sanguineous temperament, we have those characteristics which indicate an exuberance of vitality. The strong contractions of the heart, the florid complexion of the countenance, the development of physical powers, the gaiety or cheerfulness of the mind, or the occasional bursts of passion, are a few of those symptoms by which this temperament is distinguished. The greater part of these conditions are to be referred to the natural constitution of the mind, and the peculiar circumstances under which it has been placed. The full elucidation of this subject would present many new views in regard to the temperaments; but, on the presentoccasion, it is sufficient to observe, that if the character of the mind be cheerful, it will invigorate the system, if melancholic it will depress it. The mind has only one way of communicating its influence to the body, namely, through the function of respiration; and as this, or a similar apparatus, is the primary source of the continuance of existence in the whole animal and vegetable creation, it necessarily follows, that what excites or depresses its operation will excite or depress the whole system.

CCCCLXV. When any of the inferior animals have undergone excessive exercise, as, for 
example, when they have been chased or driven for a great distance, it has been repeatedly proved that the blood will not coagulate, consequently, that it is deprived of its ordinary vitality. This phenomenon will be found to arise from certain physical conditions of the blood, somewhat similar to those peculiar to depressing passions. Moderate exercise augments vitality, because the sanguineous fluid is favourably circumstanced for its more perfect oxygenation; indeed, it very often exhibits the buffy crust, as in cases of inflammation; but when this exercise is accompanied by great debility, we have other effects.

CCCCLXVI. Excessive exercise is attended by a great expenditure of the properties of the blood, and of those materials that the system has received for nutrition. Great exercise, before it gives rise to debility, is maintained at the expence of the nutritive qualities of the system, which, under the present circumstances, are more quickly dissipated than on ordinary occasions. The blood is more than usually stimulating; and, moreover, its circulation is augmented in every respect. The existence of these causes in some measure proves, that, if great exercise be long continued, the blood will speedily be impoverished. If we even suppose the lungs to receive the natural, or even a greater quantity of oxygen, this cannot supply the want 
of those materials essential to support the gradual loss of the constitution; its office is to act on the principles contained in the sanguineous fluid, and if these be deficient the usual chemical changes will also be affected.

CCCCLXVII. It is from the above causes that debility follows excessive exercise; and when that condition of the system becomes oppressive the regularity of the respiration is disturbed; and this alteration also tends to augment the general weakness, and to deteriorate still more the properties of the blood.

CCCCLXVIII. In regarding oxygen as one of the principal agents of the universe, through whose energetic influence the animal and vegetable creation are enabled to display their various endowments, 1 do not by any means imagine that the grand effects of its power are referrible to its simple diffusion or presence, but rather to its innumerable combinations with whatever is required for the ultimate ends of these systems of Nature. Whether we regard the secretions of the kidneys, mucous membranes, or the liver, or whether we examine the qualities of the blood or constitution of the solids, we observe every where acids, alkalies, and a variety of other combinations. In many chemical substances without the body these are, in many instances, inert or active acording to the quantity of oxygen which they contain. It must be allowed, that chemical 
science is even yet in its infancy; but if, thus imperfect in its analysis, it is capable of proving the general efficiency or superiority of oxygen, we may probably be allowed to anticipate that, at some future period, it will demonstrate the correctness of opinions, at present little better than conjectures, which, however, are occasionally of great utility towards the promotion of science.

CCCCLXIX. Arterial blood is rendered as dark as venous by the application of galvanism. If coagulated, on the employment of this agent it becomes fluid, and never again assumes the same condition. Individuals are occasionally killed by lightning, and in such the blood is found in a fluid state. It is not improbable that galvanism and lightning are of the same nature : their influence on the animal economy presents the same phenomena. These agents are said to destroy the vitality of the blood; and if it be supposed to be the result of oxygen and its various combinations, no opinion is better supported by experiments. There is scarcely a substance in Nature but what is almost immediately de. composed by the application of a strong galvanic power. We know that chemical combinations exist in the blood; and we still further know, that such are separated without the body into their different elements by the influence of the galvanic aura. Therefore, if the blood be considered 
vital before we employ this agent, when these chemical combinations are entire, and that it loses its vitality on the application of this power, we are almost necessitated to allow that vitality is the result of these combinations, as it no longer exhibits any of its properties wohen these are destroyed.

CCCCLXX. In Chapter VII. on Torpidity, I have dwelt at considerable length on the particular influence of cold in retarding the oxygenation and circulation of the blood; and have also endeavoured to shew in what manner warmth tends to restore the enfeebled powers of life. The diminution and increase of animal and organic functions were, in these instances, the diminution and increase of vitality.

CCCCLXXI. Nothing in Nature can be attributed to chance. Harmony and beauty are observed in the grand outlines of design, perceived in those creations within the grasp of our faculties; and since the Supreme Being has thus wonderfully constituted our frame, and given it established laws, it is somewhat probable that oxygen is the instrument which principally tends to accomplish his intentions. Regarding oxygen as a primary principle of vitality harmonizes with the figurative language of Scripture, in which it it is stated, that after God had created man " He breathed into his nostrils the breath of life.' 
CCCCLXXII. Having generally investigated the subject of vitality, we are now, in some measure, better prepared to define it. The arterial blood in the fotal germ is certainly present before evident structural adaptations, except vessels, are formed; and, therefore, the blood, or vital principle, may be said to precede organization; but it is, at the same time, the result of organization, as it is derived from the mother. The blood she distributes to this vital nucleus is of the same kind as that which is employed to secrete the gastric juice, bile, or mucus. These different secretions cannot be supposed to originate from different kinds of blood, but must be referred to peculiar organic adaptations proper to each organ, in the same mainer as the eye is fitted to the laws of light. It forms no part of our inquiry to investigate how the foetal system is developed. We do not know the laws by which a crystal is formed, which appears a much simpler phenomenon. It is our province to examine the circumstances which are closely connected with the object we cultivate. From having pursued this plan, I am disposed to attribute vitality, or the principle by means of which the system is gradually formed and supported throughout existence, to qualities of or combinations existing in the blood. It has also been seen, in the preceding pages, that these qualities or combinations have 
an exciling or depressing tendency ; or, in other words, exhibit a superabundance or deficiency of vital energy according to the quantity of oxygen which the sanguineous fluid contains. From this reasoning we are not to conclude that vitality and oxygen are synonymous; with as much consistency we might state that a machine and its source of motion are the same. The functions of the animal frame are retarded or accelerated according to the quantity of oxygen existing in the blood, or according to its changes in the lungs; and we also observe that the different machines which man employs, whatever be the power that continues their action, have $a$ force proportionate to the extent of the moving cause. But, in these examples, there is no similarity, either in the agent which operates, the materials acted upon, or the effects produced.

Life is not a simple principle, but is the result of compound principles and actions pervading and common to every part of organized nature. 


\section{CHAP. XVII.}

\section{On Sympathy.}

CCCCLXXIII. There are few subjects in medicine that have occupied, equally with Sympathy, the attention of the physician and physiologist. Whether the human frame be regarded in the state of health or disease, certain indefinite links of association seem to connect the various organs of the constitution; and the phenomena which arise from the reciprocal action of these organs, in their different states, are frequently, to the physician, indications of practice. It is scarcely necessary to enumerate the divisions and subdivisions of sympathy which have been proposed. Some men of eminence have endeavoured to point out certain intimate relations existing between individual membranes of the same or of a dissimilar description, between internal organs and the mucous or cellular membrane, or between parts that are continuous, contiguous, or remote. By some the nerves and the brain are regarded as the only efficient agents in transmitting, influencing, or in maintaining every species of irritation or disorder. 
CCCCLXXIV. It is seldom that a series of propositions is altogether true or false; and the correctness of this observation is obvious, when applied to the different theories concerning sympathy. But if these had been capable of demonstration, or had immediately enlightened our professional views, our reasonings would have been less confused and indistinct. The great objection to the prevalent opinions with respect to the laws of sympathy is, that they are either gratuitous, or suggest little improvement in practice.

CCCCLXXV. It is not, on all occasions, easy to detect error, and, when detected, it is sometimes more difficult to supply its place with truth. But, in medicine, the exposure of error, sooner or later, leads to the establishment of truth, or gives rise to principles that are more or less salutary in their influence. In this chapter I shall attempt to expose many important errors either committed or supported by physiologists of eminence; and, while I liberally bring forward the mistakes of others, I shall humbly propose opinions that appear to me better calculated. to solve the most essential phenomena of sympathy, and which also appear to have a closer connection with the indications of practice.

CCCCLXXVI. Sympathy, however produced by the different states of the body, I have divided into the four following classes: 


\section{$a,-$ Sanguineous. \\ $\boldsymbol{b}$,-Sensorial. \\ $c$,-Nervous. \\ $d,-$ Nervo-sensorial.}

1. By the term Sanguineous Sympathy, I allude to affections produced and propagated by changes in the nature, quantity, and circulation of the blood.

2. By Sensorial Sympathy, I mean certain effects originating in sensations or states of the mind subsequently propagated through the medium of nerves:-an example of this division is the production of syncope and vomiting from a mental cause.

3. Nervous Sympathy is the propagation of certain effects accomplished by nerves alone, as in risus sardonicus, arising from irritation or inflammation of the diaphragmatic nerve communicated to the seventh pair of the face.

4. Nervo-Sensorial isa term employed to explain phenomena which result from the irritation of a nerve or nerves, of which the brain takes cognizance, and which afterwards transmits its impressions to other organs of the body. Vomiting is frequently an instance of this reaction.

CCCCLXXVII. The division proposed will enable us to simplify the explanation of many irregular actions or states of the body; and if the views and principles be found correct after mature examination, they are sure to suggest an improved mode of treatment in many anomalous and well marked-affections of the system.

Sanguineous sympathy will shew, in a manner more consistently than has hitherto been 
done, why parts that are continuous, contiguous, or remote, are at times simultaneously affected; why the same or different membranes are alternately or reciprocally influenced by disease; why disease in one organ induces derangement in another; and why the aberration of one function occasionally implicates the whole system.

CCCCLXXVII. There is scarcely a cot d tion of the body that is not produced by sympathetic action ; irritations, inflammations, dropsies, fevers, delirium, hemorrhagies, and a variety of other affections, are said to be examples of its influence.

CCCCLXXVIII. The consideration of hemorrhagy, as the first illusiration of Sanguineous sympathy, will occupy the present chapter.

This disease has been divided into the active and passive; in the former, we have generally a slight degree of febrile action, an accelerated or full pulse, a sensation of heaviness or oppression in the chest or head; but in many instances the flow of blood is neither preceded nor accompanied by any of these symptoms :- the other seems to take place without an increased action of the circulation, occurring most frequently in debilitated habits or organs.

Many objections have been brought forward against this division. Although it be not strictly correct, yet its adoption is considerably less in- 
jurious in practice than many of the additions and views of those who are adverse to it.

CCCCLXXIX. Broussais regards all hemorrhagies as depending on an irritation of the capillary vessels, consequently deems them active, and endeavours to shew that debility does not occasion the flow of blood. He says, if debility produces hemorrhagy, that it ought to occur in all cases of long continued weakness, in the latter stages of life and disease; that a paralytic limb ought to be subject to the affection, which is never the case; and still further, that ecchymoses and petechiæ ought to characterize as frequently diseases of manifest debility as fevers of a typhoid nature. These arguments are far from being conclusive; his favourite theory, irritation, seems to obscure his reasoning powers, or necessitates him to adapt to it a variety of symptoms and conditions of the constitution that cannot, except by force, be incorporated with the dangerous and exclusive views implied in it.

CCCCLXXX. From the observations of Broussars, one would imagine that he looked upon debility as the same in its effects, how different soever the causes that produce it. Debility is sometimes occasioned by excessive depletion; by want of proper nourishment; by inflammatory and typhoid fevers; by slow pulmonic disorganizations, and by the gloomy passions of the 
mind; but in every one of these cases, the debility, in relation to the condition of the solids and the fluids, is as various as its causes. If we, for a moment, examine a patient labouring under phthisical symptoms, we perceive the fair complexion intermingled with a florid flush, white teeth, and snowy hands, body easily fatigued, and mind buoyant with bright hopes; but are these the symptoms we observe in thelatter stage of typhus?

CCCCLXXXI. The nature of the sanguineous fluid will vary with the character and duration of the disease; and the state of the whole system will be influenced more or less by the same causes. If the body suddenly become weak, we cannot expect that the blood, the fluids, and the solids of the system, shall have undergone those minute and extensive changes which invariably follow or accompany opposite circumstances. The healthy action of vessels may be as much deranged as the fluids which circulate in them; and when this general diseased state occurs, we certainly have a right to anticipate the well-marked indications of asthenic affections. We have then petechiæ or ecchymoses, not because the blood alone is diseased, or the capillary vessels, or the various solids or fluids of the body, but because the whole of them are more or less affected. If this reasoning be correct, is it consistent to expect in a paralyzed limb, or in the decrepid weakness of old age, or in the last moments of life, the same external signs of c c 2 
debility? Neither the blood nor its vessels are in the same diseased condition or diminished state of vitality.

CCCCLXXXII. Dr PARRy, in speaking of sea-scurvy, seems to think that it may probably in all cases be preceded by an increased momentum of blood, and, still further, that petechiæ are probably more rare in typhus than in fevers of a purely inflammatory type.*

Dr Parry's experience does not harmonize with that of the generality of observers. Petechiæ are seldom or never found in diseases of a purely inflammatory description as long as the inflammatory type continues, but are found at the termination of the excited action, when the body has become extremely debilitated by the previous excitement and derangement of functions. The severe forms of the plague are occasionally unaccompanied by inflammatory action,-and yet we perceive in this dreadful disease the most extensive consequences of asthenic influence.

CCCCLXXXIII. Dr Cullen has endeavoured to explain why different kinds of hemorhagies occur in different organs of the body at the various periods of life; but his arguments appear to me quite unsatisfactory. He says, that youth is most subject to epistaxis, because the head has its organization the sonnest completed; the consequence of which is, that the solid parts less

- Elements of Pathology and Therapeutics, p. 164. 
easily adapt themselves to a continued distending power, but, as this force is frequently exerted, a rupture of vessels necessarily takes place.

rCCCLXXXIV. Hemoptysis, at a later period of life, is supposed, by the same author, to arise from the balance being destroyed " between the systems of the aorta and pulmonary artery, or between the vessels of the lungs and those of all the rest of the body."

CCCCLXXXV. As hemorrhagies of a passive character are often remarked at a time of life muchlater than the two preceding, he has brought forward a truly ingenious hypothesis to explain the origin of these diseases ; but it is not, perhaps, less chimerical than the former reasons he adduces. He observes, that the veins in early life have a greater proportional density than the arteries, and that on this account they are enabled to propel with greater energy their contents into the latter, and in this way they subsequently become distended or engorged, giving rise to epistaxis and hemoptysis; but, towards the decline of life, the arteries have their cellular texture increased by distention, and from this cause they acquire a greater proportional density than the veins, so that these are liable to receive more blood than they can possibly circulate.

CCCCLXXXVI. In place of explaining the preceding phenomena on the supposition of this or that organ being the soonest completed, of 
that the coats of the vessels are subjected to the modifications he imagines, I prefer regarding the three periods of life as periods of constitutional changes in the mode of circulation, and in the degree of the vitality of the blood, independent of the organic alterations of which he speals.

CCCCLXXXVII. In early life the circulation is quick, frequent, and regular; the temperature of the body is higher and more permanent than that of the adult in the decline of years; the respirations are often repeated, and from the restless mind of youth, and the variety of desires and wants, the physical powers are continually exercised. These conditions tend to develope and strengthen the various organs of the body; the assimilating functions are more vigorous; and, from these causes, the whole arterial system exhibits a degree of energy superior to that of the veins.

From the peculiarities of youth, the colour of the countenance, the temperature of the body, and its unceasing exercise, the conditions of the circulation at this time of life are designated by the term external, in contra-distinction to its general distribution in the later stages of existence.

Taking this view of the subject, epistaxis is regarded as the effect of an increased action of the whole sanguineous system, conjoined with a local determination of blood greater than what is consistent with the proper operation of the organic functions. 
The complicated net-work of vessels which covers the internal surface of the nostrils, offers an easy passage to the blood when its momentum is suddenly increased; and as no other part of the body is similarly constituted, bearing the sume relation to this external circulation, the blood will flow from the nose in preference to any other part of the system.*

CCCCLXXXVIII. Dr Cullen says, that "an hemoptysis may be expected to happen from a faulty proportion between the capacity of the lungs and that of the rest of the body." This reasoning appears perfectly correct; but I cannot perceive the justness of what follows, "that an hemoptysis will especially happen about the time that the body is approaching to its acme, that is, when the system of the aorta has arrived at its utmost extension and resistance." According to this opinion, hemoptysis does not occur in childhood, because the aorta accommodates itself to every additional quantity of blood. The thoracic viscera in infancy and childhood are extremely active, and from the great expirations and violent exertion of individuals at a tender age, we should certainly anticipate derange-

* It may probably be asked, Why does it not flow from the anus? In answer to a question of this kind, I may observe, that the mucous membrane of the nose is highly vascular, as the seat of a sense; and, from its proximily to the thoracic organs, more liable to be disturbed by those causes that increase the impetus of the blood. 
ments similar to what take place at the acme of life, if the extensibility and resisting power of the orta and pulmonary artery were the only or chief differences of the two periods.

There are three reasons which induce me to dissent from Dr Cu I EN's explanation :

$a$,-The comparative unfrequency of hemoptysis is one of considerable importance. If we suppose, agreeably to him, that certain great organic and functional changes take place at any two or three periods of existence, and that these are not peculiar to one individual, but are common to all mankind, from the operation of universal causes on matter precisely of the same nature, is it philosophical to bring forward a few solitary cases in support of principles that are found inefficient in their general application?

$b$,-The second objection to this view is, that the aorta and pulmonary artery are sometimes found to be partly ossified without this condition having been characterized by hemoptysis, or any unpleasant symptom. When the parts are thus disorganized, the distending power and extensibility must be materially diminished.

$c$,-In the third place, Hippocrates has stated, that hemoptysis happens between the age of 15 and 35 ; and to this observation Dr CuLLEN assents. If hemoptysis depend on any particular conformation of an organ or organs, or on any constitutional change that occurs, or is liable 
to occur at one part of life called its acme, it is impossible or absurd to suppose that this same acme shall at one time be present at 15, and at another at 35 years.

CCCCLXXXIX. After having weighed maturely the reasoning of Dr CulLEN, and having also reflected on the revolutions of the system,on their concomitant circumstances or subsequent effects - I am persuaded that there are changes in the mode of circulation in every period of life, which satisfactorily account for their accompanying or succeeding phenomena, without referring these changes to the organic development or alterations of which he speaks.

From youth to manhood the circulation has evidently undergone great modification; the respirations are less frequent, and various organs begin to assume a new or more important character in the animal economy. The development and support of these organs must necessarily make a call upon the sanguiferous system, and in proportion as the demands of the internal functions are increased, either by exercise or the evolution of new powers, in the same ratio the general or external distribution of the blood will be diminished.*

CCCCLXC. The condition of the circulation at this period I would call internal, not wishing to imply, by this expression, a state ap.

* Vide Chap. IV. 
proaching to congestion, or, at the same time, that the surface of the body is deprived of its necessary blood, but simply to communicate to the mind an idea of a mode of circulation peculiar to this stage of life.

CCCCXCI. Exertion, of whatever kind, has a tendency to augment the flow of blood; and, whenever its motion is facilitated, a greater quantity, in a given time, is transmitted to the lungs with augmented impetus; and these, from their organization and office, are calculated to experience the first pressure made upon the system at the different periods of life.

CCCCXCII. If we take into consideration that the lungs, in some individuals, are predisposed to hemorrhagy, or that the circulation of blood, or that the activity of the internal organs is naturally different or variable, it is not difficult to explain the occurrence or absence of hemoptysis.

CCCCXCIII. Hemorrhoids, hemorrhagia ani, and diseases of the same kind, which are called passive, from the veins being the seat or source of these, are accounted for by Dr Cullen in the following manner:

"The proportional density of the coats of the veins to that of the cuats of the arteries, is greater in young than in old animals. From which it may be presumed, that the resistance to the passage of the blood from the arteries into the veins is greater in young animals than in old; and, 
while this resistance continues, the plethoric state of the arteries must be constantly continued and supported. As, however, the density of the coats of the vessels, consisting chiefly of a cellular texture, is increased by pressure, so, in proportion as the coats of the arteries are more exposed to pressure by distention than those of the veins, the former, in the progress of the growth of the body, must increase much more in density than the latter; and, therefore, the coats of the arteries, in respect of density and resistance, must come in time not only to be in balance with those of the veins, but to prevail over them."*

CCCCXCIV. The preceding paragraph of Dr Culles is beautifully ingenious ; but, if examined critically, will be found to be hypothetical or fallacious. The reasoning is intended to show why hemoptysis does not happen after the age of 35 years, and why the veins ultimately become the seat of an affection similar to that which has previously existed in the arteries. To suppose, in the first place, that the coats of the veins, in early life, have a greater proportional density than those of the arteries, in order to account for the arterial flow of blood, is scarcely consistent with the phenomena we observe in the animal economy.

* Cullens First Lines, Vol. II. dcclevi. 
CCCCXCV. At the period of life when he imagines the veins to obstruct the current of blood flowing from the arteries, in consequence of which the latter are distended, the pulsations of the heart are frequent and strong,- - the respirations are numerous, - the physical powers are continually exerted, and every other means that can in any way increase the respiratory and circulatory functions are present.

CCCCXCVI. It is the excited state of the arterial system, and not its engorgement from the obstacles which the venous presents, that occasions the phenomena of arterial hemorrhagy. That the coats of the arteries acquire additional density from the power of dislention, is a supposition that gains little support from the operation of the same agent on other parts of the system.

CCCCXCVII. The heart is not only unusually dilated, but even enfeebled by the distending force of the blood. The bladder, the intestines, and the stomach, are debilitated by the influence of such a power; nor am I acquainted with any organ of the body that is augmented by distention alone. The heart, when its parietes are thickened, has been subject to an increased action, which has a tendency to stimulate its own individual vessels, and these receiving a greater quantity of blood, or blood of more perfect oxygenated properties, give additional nourishment to its muscular fibres. 
CCCCXCVIII. If we reason on the influence of distention in the arteries themselves, it is difficult to conceive how this circumstance can produce a greater degree of tonicity or strength. The distention of a vessel implies the enlargement of its cavity; and whenever this condition occurs, the circulation is retarded.

CCCCXCIX. Instead of the veins becoming the seat of hemorrhagy from the great ar proportional density of the arteries, they are this circumstanced owing to the arteries hemelves haring their ncrmal cation deranged. If the respirations are less perfect, and the heart becomes less able to propel its contents, the veins must participate in the deteriorated action. In advanced life, when the symptoms of passive hemorrhagy occur, there are many proofs present, which indicate that the abdominal congestions are in accordance with the diminished activity of the thoracic functions. The pulse is generally slow, full, and soft, and the individual does not possess the disposition or ability to exert those physical powers that act as stimuli to the sanguiferous system. Agreeably to these principles, we perceive, that many debilitating causes, even before the age of 35 , are known to occasion the ordinary effects of old age, by acting in a similar if not precisely in the same manner upon the arterial and venous circulation.

D. The explanation which Doctor CuLLEN gives of the preponderance of the venous 
system towards the decline of life, as accounting satisfactorily for the origin of apoplexy, appears to be exceptionable in its principles. The knowledge of those organic states that are known to predispose to this disease, and an investigation of those causes that hasten its occurrence, would certainly lead one to believe that the short neck is favourable to the flow of the arterial blood towards the head, and that every accidental and continued agent which tends to promote the affection acts powerfully upon the arterial system, increasing the rapidity, force, and preponderance of its actions. But Dr Cullen supposes that apoplexy takes place "when the powers promoting the motion of the blood are much weakened."

DI. A poplexy is not a disease that is particularly confined to the decline of life ; but, in many instances, if not in the greater number, occurs when an individual is in the full enjoyment of his mental and corporeal faculties. Nor does it happen to those whose venous system seems to prevail over that of the arterial, but when this has the ascendancy, or at least when it is much excited. Nor, in the last place, does the mode of living that predisposes to an attack of apoplexy appear to occasion, in an exclusive manner, an equable or general turgescence of the veins, except as a consequence of increased arterial action. 
DII. The question has frequently been agitated, How hemorrhagy contributes to a plethoric state of the system? The fact can scarcely be doubted : and the knowledge of it has diminished materially the long-established custom of annual depletion. It is observed by Dr Cullen, " that the quantity of serous fluids being given, the state of the excretions depends upon a certain balance between the force of the larger arteries propelling the blood, and the resistance of the excretories; but the force of the arteries depends upon their fulness and distention, chiefly given to them by the quantity of red globules and gluten, which are, for the greatest part, confined to the red arteries; and, therefore, the spoliation made by a hemorrhagy being chiefly of red globules and gluten, the effusion of blood must leave the red arteries more empty and weak. In consequence of the weaker action of the red arteries, the excretions are in proportion diminished, and therefore, the ingesta continuing the same, more fluids will be accumulated in the larger vessels."

DIII. If the above explanation be correct, one would imagine that the ordinary means which contribute to create a state of plethora would be similar to those that produce a sudden return of that condition. But we find that individuals whose pursuits require great exercise, and whose mode of life is extremely simple, are not unusual- 
ly stout and plethoric. Constant exercise promotes the circulation, and increases the action of the excretories. The quantity and quality of the ingesta appear to have less influence in inducing the effect than is generally supposed. If we even granted to Dr Culles that the action of the excretories depends on the force of the sanguineous fluid, it would enable him to solve the phenomena connected with the present subject; but it would certainly give rise to new difficulties. The circulation of the child is particularly frequent, and the motion of the heart strong for its years, and yet it acquires, in a very short time, a plethoric condition of the constitution.

DIV. Since the time of Dr Cullen, physiological science has introduced many improvements, and these enable us to appreciate more correctly the discoveries and the theories of preceding ages. Much additional light has been thrown on the intricate subject of absorption; which seems to prove that the vessels appropriated to this function are exiled or depressed according to the fulness or distention of the sanguineous system.

DV. Depletion diminishes the force or fulness of the arterial circulation; and when this result is produced in the state of health, from a law of the animal economy, the absorbents are enabled to take up more nourishment in a given time, and it is this augmented power that so 
quickly brings on the train of symptoms which the lancet had previously removed, and not the diminution of the excretions, the usual quantity of the ingesta remaining the same, as imagined by Dr Culden.

DVI. The influence of heat and cold upon the body shows the extensive sympathy which is experienced by every part of the system in consequence of the changes of circulation. When heat is applied to the surface of the body, it stimulates the capillary vessels to increased action; and this condition is communicated to the larger vessels, and afterwards to the lungs themselves. When the heart and the respiratory function are indirectly excited, as in this instance, they react upon the sanguineous fluid, and transmit it in greater abundance to the capillary vessels. During this reciprocal action, the remote and external parts of the body receive a greater quantity of blood; but the rapidity of the motion with which it circulates in the lungs over excites or disorders the minute vessels, which chiefly compose their substance, and gives rise to hemorrhagy. Stimuli taken within, and exercise of every description, produce the same effect. If we consider that the organization of the lungs is extremely delicate, and that the capacity and operation of these must be in harmony with the different branches of the arterial and venous cir

v d 
culation, yet at the same time are subject to every alteration of the sanguiferous system, the absence, and not the occurrence of hemoptysis, becomes a matter of surprise.

DVII. It was formerly believed that hemoptysis was the consequence of a rupture of some of the pulmonary vessels; but modern pathologists have clearly shewn that this circumstance seldom happens except when a vessel, traversing a tuberculous excavation, is partly destroyed by the progress of disease. Hemoptysis is sometimes the consequence of an aneurism opening itself into the trachea, bronchia, or substance of the lungs.

DVIII. When the quantity of blood which is poured from the lungs is even considerable, it is regarded by LANNEC as a sanguineous exhalation into the pulmonary parenchyma, and is described by this pathologist under the name of Pulmonary Apoplexy.*

DIX. Cold, and the sudden removal of atmospheric pressure, are enumerated by CULLEN among the exciting causes of hemorrhagy. It is almost impossible for the former to produce an active form of the affection, as this does notdepend, as is supposed by some, upon a state of congestion determined by the slow and gradual influence of cold, but is more justly to be referred to an increased action of the capillary vessels. In

* Lenvec del Oscultation Mediate, Vol. II. p. 45. Edit. Prima. 
apoplexy, it is probable that great congestion is present. We have frequent proofs that the disease, at times, consists entirely in an over-, charged condition of the vessels of the head.

DX. The reasoning which is employed to explain the origin of hemoptysis or epistaxis, would be incorrect if applied to explain the phenomena of apoplexy. The seat of the latter is the brain, which is enclosed by the unyielding parietes of the skull, and is supposed by Monro and Dr KerLIE to occupy fully the cavity in which it is contained, It is therefore obvious, that an accumulation will easily take place, and this, though existing to a slight extent, will be immediately indicated by the symptoms of compression.

DXI. Epistaxis does not arise from a simply congested state of the vessels, but from the action of these being augmented. Nor can we attribute hemoptysis to a similar condition of the pulmonary vessels. A great number of thoracic diseases retard the flow of blood in the lungs without the congestion induced being accompanied or succeeded by hemorrhagy. If we review the ordinary exciting causes of hemoptysis, we shall discover that it is the tendency of such not to weaken or obstruct the flow of blood, but to facilitate its motion, increase its strength, and to give addilional slimulus to its character. From these, and similar considerations, we may con,

p d 2 
clude, that epistaxis and hemoptysis have not the same proximate cause as apoplexy.

DXII. It is stated, that the sudden removal of atmospheric pressure distends the external bloodvessels in the same manner as heat. There is a certain seeming coincidence in the phenomena that these present: but if wenarrowly investigate thecircumstances in which they resemble each other, we shall perceive a great difference in the origin and influence of their peculiar properties. Heat does not simply distend the blood-vessels, but more particularly rouses the circulation, and gives rise to those general consequences described in $\mathrm{DV}$., but particularly in Chapter VIII. The removal of atmospheric pressure does not immediately stimulate the vessels upon the surface of the body, but allows the blood to flow here with greater facility, and gives to the vessels a degree of fulness and distention, not unlike the appearance exbited by an increase of temperature; but when such is the consequence of heat, the internal organs are not only relieved by a diminution of blood, but are likewise excited by the stimulus conveyed to the respiratory and circulatory functions; but, in case of diminished pressure, no stimulus whatever is communicated to these, except such an improvement of cnergy as may follow the change in the distribution of the blood.

DXIII. Philosophers not being fully acquainted with the alterations which the circulation 
undergoes on the removal of pressure, when the body is completely at rest, or when the whole system is animated by exercise, have differed among themselves as to the precise operation of this power. When an individual ascends one of the loftiest mountains, he has great difficulty of breathing. The countenance is extremely flushed, and the veins on the surface of the body are much swollen. These and a variety of other symptoms are said to be the results of diminished pressure; but when the aeronaut is elevated to an equal height, he does not experience the same painful sensations, nor does the system exhibit the same effects. The evident difference in the consequences, when the body is similarly exposed to the influence of the same agent, is a circumstance which militates materially against the rationale proposed by the most enlightened physicians.

DXIV. The nature of these physiological studies have naturally led me to investigate the influence of pressure, when exerted alone, and when in combination with exercise; and the principles deduced appear to harmonize the diversities of opinion previously entertained on this subject. When the body is in a state of repose, and subjected to a cold atmosphere, which is always the case with the aeronaut, the flow of the blood becomes more internal than external, in consequence of which the diminished pressure has to act upon a less quantity of sanguineous fluid, 
and has also to contend against the influence of the cold, whose property it is to constrict the capillary vessels, and, by lessening their diameter, to retard the flow of blood to the surface of the body; but when this condition of atmospheric pressure is conjoined with exercise, the influence of the cold is counteracted. The respiration and circulation are much accelerated by exercise, as is universally known, and therefore the blood is transmitted in greater quantity, and with superior energy, to every part of the system. It is now external in its distribution, and the effect of the atmospheric pressure is materially augmented by the addition of a new power acting in unison with its own. A greater quantity of blood being determined upon the surface of the body by exercise, and the influence of the diminished pressure, the consequence is, that the proportion of this fluid in the lungs is materially lessened; therefore its stimulating properties and circulation reill be much increased. Many statements are on record which clearly show that the union of the two causes has been attended by epistaxis and hemoptysis, and many other symptoms of sanguineous disturbance; and the rationale proposed seems to be satisfactory in the solution of the phenomena. The fact that hemoptysis and epistaxis occur under these circumstances is strongly corroborative of the idea concerning their proximate cause.*

* Vide Appendix. 


\section{CHAP. XVIII.}

The general action of Emetics on the system, with a few remarks on their efficacy in Chronic and Acute Diseases.

DXV. THERE are few classes of medicines that have been so universally employed as that of emetics. From the earliest authentic records of medical science to the present day these substances have been used, and occasionally extolled as specifics in a variety of diseases. It is impossible to deny the good effects which have been attributed to them; and it is not difficult to account for their success and general adoption at one time, or for their injurious tendency and partial reception at another.

DXVI. When our knowledge of the operation of medicines is narrow or inaccurate, or when we are, in a great measure, ignorant of certain important laws of the animal economy, our practice is necessarily empirical. If, at one time, we have cured diseases of a certain class by emetics, at another we immediately prescribe the same, whenever the same or similar indications are present, without being fully sensible that the means which we had previously found beneficial were complex in their nature, and that the affections which we had to treat were also as multifarious 
and variable as the seasons, the conditions of the body, the severity and number of the deranged symptoms, and many other modifying circumstances which had not been duly considered.

DXVII. We are too frequently misled by what may be called the outline of diseases, and too littleregulated by what may be considered the subordinate features or symptoms of general or local disorders. He that, at one time, cures by wholesale, will, at another, destroy in the same proportion. His knowledge is the only fixed and unvarying object: every thing around him is continually changing; and it is therefore obvious that the application of his principles will be efficient or injurious according to the conditions of the system.

DXVIII. The subject of this chapter will be a further illustration of sanguineous sympathy ; and I am not aware that any other class of medicines so directly proves the justness of the division of sympathies I have introduced as the one under consideration. By the exhibition of emetics the constitution is roused or depressed to an extreme, and the most characteristic phenomena attending these different states belong to the sanguiferous system.

DXIX. The action of emetics on the animal economy might have been deduced from the general principles developed in different parts of this work; but I have, on the present occasion, endeavoured to demonstrate, by direct experi- 
ment, the nature of this action. In this undertaking I was kindly assisted by my friend $\mathrm{Mr}$ -Buchan of Devenport.

DXX. I was induced to try the effects of emetics for the purpose of ascertaining clearly the state of the circulation during nausea and after vomiting, and also of noting the alterations which these produced upon the temperature of the body. I took four grains of emetic tartar, dissolved in seven or eight ounces of water. At the commencement of the experiment the temperature of the room was $59_{\frac{1}{2}}{ }^{\circ}$, the pulse 77 , full, and of good strength: the heat of the body was $97^{\circ}$. In the space of half an hour I felt slightly sick, but did not experience the least chillness or rigor. To facilitate, if possible, the progress of nausea, I took, in a very short period, between three and four pints of warm water. This quantity of liquid quickly brought on, to a trifling extent, the unpleasant sensations of sickness ; but, before these became painfully disagreeable, vomiting was occasioned, but lasted only for a few seconds. After the copious ejection of the watery contents of the stomach, the pulse was 100 , very weak and small, and the temperature of the body had risen to $1001_{\frac{1}{2}}^{\circ}$. In fifteen minutes after the evacuation of the contents of the stomach, the pulse was found to be 80 in a minute, small, but not weak, and the temperature of the body was $99^{\circ}$. In an hour and a quarter from the commence- 
ment, when I experienced no nausea or disposition to vomit, but certainly felt great debility or weariness, the pulse was still about 80 , and the animal heat remained stationary at $98^{\circ}$. I had to regret, in this experiment, that the nausea had not been sufficiently severe to cause its ordinary and well-marked effects upon the system. To obviate this incompleteness, Mr BuchaN very kindly submitted to a similar experiment, and the general results were in every respect the same as those already detailed, with the exception of the nausea; and this continued in him, oppressive and disagreeable to an extreme, for almost three hours,

DXXI. At half past eleven o'clock, A. M. the day succeeding the previous experiment, his pulse was 66 in a minute, small, the temperature of the body $98^{\circ}$, and that of the room $63 \frac{1}{2}^{\circ}$. These circumstances being accurately noted, he took four grains of emetic tartar, in about eight ounces of water. He did not feel the least inconvenience from this quantity for more than half an hour; and, being desirous to promote sickness, he took 10 grains of ipecacuanha in a similar quantity of water; but this was accompanied by no unpleasant sensation. On this account the same dose was repeated; and, in three quarters of an hour from the commencement of the experiment, he complained of great coldness in the back and the superior and inferior extremities, 
particularly the latter; in fine, so severe was the sensation of cold, that he employed muscular exertion for the purpose of warming himself. Great sickness almost immediately succeeded this state of feeling, and, during its continuance, the pulse was 74, very weak and small, and the temperature $96^{\circ}$. After he had vomited several times, the pulse was $\mathbf{7 7}$, still very weak and small, but the animal temperature had risen to 98!. Nausea, with occasional vomiting, continued until one o'clock, at which time the pulse was 74, presenting the above characteristics; but the thermometer, when applied to the mouth, as usual, indicated $99^{\circ}$.

DXXII. Before I draw any conclusions from these facts, I may state certain peculiarities connected with the mode in which the thermometer was influenced in the case of $\mathrm{Mr}$ Buchan. Before the experiment, the instrument remained several minutes in the mouth, without the mercury being raised so immediately as it generally is to its usual point. To make the instrument as sensible as possible, the mercury was raised to about $98^{\circ}$ before it was applied; yet, notwithstanding these means, it still required several minutes to raise it to the few remaining degrees. When it indicated at one time $96^{\circ}$, it seemed to demand a still longer period of time before it attained this point upon the scale; but after vomiting, when the thermometer stood, at one mo- 
ment, at $98 \frac{1}{2}$, and at another at $99^{\circ}$, the sensibility of the instrument appeared proportionately augmented. The gentleman, to whose animal temperature I allude, is young, vigorous, and apparently of strong constitution ; his chest is very well developed, and he has not been subject to any pulmonic disease whatever; yet, notwithstanding these organic adaptations for the generation of animal heat, and for the force and fulness of the pulse, his temperature is generally beneath the average, and it is with the greatest difficulty that he can bear cold. His pulse ranges from 60 to 65 in a minute, and is at all times small.

DXXIII. Having, by the preceding experiments, observed the operation of emetics upon the system, in reference to certain preconceived, and, I almost venture to say, established principles, I hope the attempt will not be deemed presumptuous that aspires to regulate in future the exhibition of emetics, by explaining, in a clear and simple manner, the nature of their operation, the organs or systems directly influenced, and those that are secondurily acted upon by the changes induced.

DXXIV. Emetics may be divided into two kinds; into those which nauseate, and into those which excite vomiting. 'The former have always been considered as depressing the circulation, and the latter as stimulating it; but it has never been sufficiently ascertained in what way this is at 
one time depressed and at another stimulated. It would seem that the nervous system occupied a prominent place in the minds of those who endeavoured to explain the series of consequences, as they frequently speak of sympathy being exerted between different parts of the system.

DXXV. In the two experiments performed to elucidate the operation of emetics, the pulse invariably became small and weak in the stage of nausea, and when this was very severe, it was almost imperceptible; it would also appear that the animal heat is equally affected by those causes that diminish the force and increase the frequency of the pulse. In my own case, after vomiting, the thermometer rose from $98^{\circ}$ to $100_{\%}^{10}$, and in Mr Buchan's from $96^{\circ}$ to $99^{\circ}$.

DXXVI. The perspiration which follows vomiting has been attributed, by almost all writers on the subject, to a particular sympathy existing between the skin and the stomach, or between eertain internal organs. Dr Cullen observes, "Thus we are of opinion, that there is a special consent between the stomach and the vessels on the surface of the body, so that the several states of these are mutually communicated to one another; whence the action of vomiting excites particularly the action of the vessels on the surface of the body."

A more modern, and a highly distinguished author, remarks, - "The feeble state of the circu- 
lation, as indicated by the pulse, and the general coldness and languor experienced during a paroxysm of nausea, are to be ascribed to those sympathetic relations by which the brain, stomach, and heart, are reciprocally influenced." *

DXXVII. Although I cannot subscribe to the above opinions, yet it is with diffidence $I$ dissent from such respectable authorities. The coldness which accompanies the operation of emetics, arises from the same proximate cause as that rehich is the consequence of an exposure to a low state of the atmosphere. The blood, in the latter instance, leaves the extremities and the surface of the bory, because the cold constricts the capillary vessels, on which account these contain less and the internal organs more blood. In the former, the ordinary mode of circulation is disordered, and this necessarily communicates its derangement to the respiratory functions; and as the alternate action of these promotes the return of the sanguineous fluid to the chest, or facilitates its motion to the different parts of the system, it is quite obvious, that whenever those means which bring the blood to the chest preponderate over those which should relieve it, an accumulation will follow 3 and it is this accumulation which satisfactorily accounts for the frequency and smallness of the pulse, the sensation of chillness, and the

* Paris' Pharmacologia, Vol. I. p. 154. 
oppression sometimes experienced in the precordial region.

It is not at all essential to the argument that the circulation be primarily deranged, and that it subsequently react upon the respiration; it is probable that the latter, in the greater numiber of instances, is, in the first place, influenced by the transmission of impressions to the mind,- the consequence of which is, that the circulation becomes irregular from depending exclusively on the conditions of the respiratory function.

DXXVIII. As nausea increases, the pulse becomes more rapid and small, till at length it is scarcely perceptible. The pale and collapsed appearance of the face, and the indications of suppressed or incomplete circulation on the surface of the body, are proofs that the blood is internal rather than external, in relation to its usual distribution. The lungs receive more blood than they are accustomed to receive ; and if the quantity be much greater than usual,it is clear that the heart will have to propel an augmented proportion, but this is evidently less arterialized from the increased quantity of blood in the chest, and therefore the stimulating principle is diminished.This is the reason why the heart does not contract and dilate energetically; but the necessity of continuing to act still remains, and consequently the blood is propelled in a small and gentle stream. 
DXXIX. This view is much strengthened by finding the pulse quicker, and still small, immediately after the first act of vomiting. During the continuance of this operation, the return of the blood from the head, and from other parts of the body to the lungs, is retarded; but as soon as the vomiting subsides, it flows in increased quantity to the chest. If, on one occasion, the pulse is small and weak, because the quantity and quality of the blood are altered, we ought to expect, on another, that these phenomena would become more apparent when the influence of those changes that produce these conditions is particularly exerted. I have shown that during nausea the pulse has certain well marked symptoms of debility, and have also endeavoured to explain their origin. If the rationale be deemed satisfactory, and if it be still further allowed, that $a$ greater quantity of blood flows to the chest after the first act of vomiting, the increased frequency of the pulse is not only in harmony with the opinion proposed, but tends materially to strengthen the principles on which it is founded.

Vomiting is always accompanied by great muscular exertion, and the co-operation of this cause is another, and probably a more efficient reason, to be assigned for the increased frequency of pulse immedialely after the emetic effect.

DXXX. I have endeavoured to depress the force and increase the frequency of the pulse by 
taking very deep inspirations, and, by keeping up a series of these for one minute, I have at all times produced the effect. In the space of that period the pulse has been augmented twelve pulsations, and its fulness and strength have been proportionally lessened. This simple experiment is not performed without being succeeded by considerable pain in the chest : it is to me sufficiently disagreeable to prevent a frequent repetition of it. Our voluntary attempts can make only a distant approach to those efforts and consequences which, are referred to the involuntary powers; but if we understand correctly the nature of those means that make even an approach to an involuntary sequence, we are certainly enabled to discriminate much betler the action of those powers that are independent of the will.

DXXXI. The explanation which has been proposed appears more philosophical than the one which assumes that there is a particular sympathy existing between the brain, stomach, and the heart. If the one proposed for the consideration of the impartial reader be granted to be true, it immediately regulates our practice; but if the other be preferred, which informs us of certain connexions existing between different internal organs, without, in the least, explaining these connexions, we are still necessitated to regard these phenomena as enveloped in the myssery of occult and impenetrable causes; and, 
from such an imperfect knowledge of some of the most important laws of the human constitution, it is impossible to extend the limits or im- prove the principles of our profession.

DXXXII. The reasoning which I have hitherto brought forward in support of the views as explanatory of the various states of the pulse, of the origin and influence of sensations, and of many important symptoms connected with the system during the continuance of nausea, is entirely founded on sanguineous sympathy. By a similar kind of investigation, and by the application of the same reasoning, we shall understand every other change in the circulation of the blood, and in the generation of animal heat, consequent on the action of vomiting.

I have already stated, that the blood from the head, and from other parts of the body, is retarded in its flow towards the chest when the stomach is engaged in the ejection of its contents; and, still further, that, as soon as the operation ceases, the blood rushes in greater quantity to the thoracic cavity, producing a condition of the lungs almost approaching to congestion; but if the vomiting be excessive, and frequently repeated, we have different results. The muscular exertion which the frame undergoes, in a succession of expiratory actions, has the tendency to equalize the distribution, and increase the stimulating qualities of the blood. The blood is more generally diffused throughout 
the system, the superficial vessels and the extremities receive an additional quantity, on account of the impetus with which it is propelled; and the pulse is also strong and full from the same cause, conjoined with the better oxygenated state of the blood. These views have been elucidated at length in various parts of this work, and, therefore, do not require a particular consideration on the present occasion. As a further support to these principles, I may mention the increase of the temperature of the body after vomiting. The diffused glow which is felt, and the profuse or gentle perspiration, cannot correctly be attributed to any other causes than those which belong to sanguineous sympathy, viz. increase of temperature, and a more equable distribution of the blood.

DXXXIII. Having thus detailed, and attempted to explain the different kinds of phenomena which originate from the two effects of emetics, it may probably, to some, appear quite evident, that, as the one excites, and the other depresses, or, in other words, as the former equalizes and stimulates the circulation, and the latter enfeebles the force of this system, there is little difficulty in applying this knowledge to practice. But medicine is a science that demands deep thought and circumspection; the principles by which we are guided are necessarily complex, and too frequently fallacious. And, as we have to act on animated matter, we must pause and consider

E e 2 
still further a few other points of this important subject, before we enforce the application of any part to practice. As nausea has the tendency to bring the blood from every part of the body to the chest and abdomen, it seems reasonable to suppose that this effect ought to be induced in cases of apoplexy or cerebral congestions, in order to relieve the head. But if we reflect that the deteriorated quality of the blood is equally as detrimental to the brain as a simple accumulation, we shall perhaps think otherwise.

DXXXIV. In the condition of the lungs during nausea, the blood is certainly distributed in less quantity, and with diminished force, to the brain ; but, as this is not perfectly arterialized, it will increase the lethargic symptoms which are present, as these arise, in part, from the deteriorated fluid, and will, most probably, at length contribute to engorge the brain, by preventing the free return of the blood.

DXXXV. The division of the effects of emetics into those that nauseate, and into those that occasion vomiting, has long guided physicians in the selection and employment of such medicines; but it has long appeared to me that this division is insufficient to regulate our practice, and there fore the following is introduced as more definite in its application. Emetics are found beneficial in a variety of diseases that have few symptoms in common with each other ; and from reflecting 
on the nature of these, and on those agents which influence them individually, it seems more just to divide the effects of emetics into four classes, and to arrange under each a few of those diseases which are obviously benefited by these effects. The first class is-

The changes both in the distribution and quality of the blood, as a consequence of the influence of emetics; and the diseases arranged under this head are:
1. Fever.
2. Dysentery.
3. Passive Hemorrhages.

CLass 2. Improved action of secernent vessels.

1. Croup.

2. Indigestion.

3. Diarrhœea.

4. Phthisis?

Class 3. The changes in the properties of the blood.

1. Active Hemorrhages.

2. Pulmonic Inflammation.

CLAss 4. Increased action of the absorbents; in which division we have the class of dropsies arising rather from debility than evident inflammation.

I am fully sensible that this division of the effects of emetics is not correct in all its parts. It 
would be absurd to suppose, that any individual system of the body is exclusively acted upon by the operation of general causes; but it would not be less absurd to imagine that every function is equally affected by them. The diseases which have been enumerated differ widely in their proximate and remote causes, and they also occupy different textures or organs of the body. Although these circumstances be so dissimilar, yet emetics have been employed in all of them, and with benefit. It is the object of the present chapter to endeavour to explain the principles on which emetics have been or may be useful.

DXXXVI. The changes in the distribution and quality of the blood are conditions common to almost every disease, but those enumerated under this head are a few of such as exhibit these changes to a greater extent than any other; and I conceive that emetics are useful only in such, as they tend to alter that distribution, or improve the quality of the circulating fluid. 'That these and subsequent views be properly appreciated, it is necessary that the proximate cause, symptoms, and treatment of each disease be generally understood; but as these considerations would interfere with the present inquiry, I shall examine them as briefly as possible.

DXXXVII. Emetics are frequently exhibited at the commencement of contagious or intermittent fevers. Few remedies are so powerful in 
cutting short the former, and I am not acquainted with any, when given before the paroxysm of the latter, that so generally stops or modifies the progress of the symptoms. When an individual is supposed to be affected with fever of whatever kind, the pulse, the respiration, the animal heat, and the blood, shew unequivocal indications of disorder, and, at the same time, some of the internal viscera are moreorless congested or inflamed. I have already endeavoured to shew that vomiting tends to equalize the circulation of the blood, and it is almost impossible to attain this end without changing at the same time the nature of the fluid. If both objects be accomplished, the affection is cut short before it is fully established in the system; the excess of animal heat which is generated by the disease itself, and by the means employed, passes off in copious perspiration, or in the other increased secretions of the body.

DXXXVIII. Dysentery is a disease concerning whose proximate cause there is little unity of opinion. Some are disposed to believe, that the larger intestines are in a state of constriction or spasm, and therefore consider this as the proximate cause. I am inclined to believe, that such a condition is to be regarded in the light of effect and not cause; others, from perceiving, on all occasions, inflammation, or its consequences, believe the proximate cause to be in- 
flammation of one or other of the membranes that form the intestines. How much soever distinguished individuals may differ among themselves on this subject, it is probable that few, if any, will dissent from the view which I take in explaining the action of those agents that occasion the disease; nor will they, perhaps, consider the outline which I give of the pathology of the disease incorrect, if they reflect on the results of dissection in such cases ; and still less, if they investigate the operation of those means successfully employed in its cure. Dysentery is most frequent in autumn, and from this reason is thought, by some, to be caused by the abundance of fruit which this season particularly affords. The human constitution must at all times adapt itself to the great changes of the year. The respiration and circulation,- - the secretions and other organic functions-have not the same action in winter that they have in summer; and, therefore, one or other of these is liable to be disordered, if these alterations be too severe or too mild for the proper performance of its office. In summer the pulse is quick and full, the animal heat higher than in any other season; and if we are to judge from these and other well-marked phenomena, we should state that the circulation is external rather than internal at this period, or that the blood is more generally distributed over the surface of the body. In autumn, there are, occa- 
sionally, great changes in the hygrometric condition of the atmosphere, in the degree of cold, and also in the frequency and severity of the winds.

If these changes be sudden or extensive, the blood will necessarily become internal rather than external in its circulation, not from a regular diminution of those stimuli or excitants that maintained the former state, but from an abrupt sup. pression of them; and as the organs on which the blood is driven cannot instantaneously adapt themselves to this unusual quantity, their individual functions are deranged. The mucous membrane of the intestines in dysentery is invariably diseased. It is not improbable, at this season, that this membrane is more susceptible of the influence of those agents whose action I have endeavoured to explain than at any other, from the tendency which fruit, and indulgences of the seasons, have to disorder its functions. If it be granted, from this short and incomplete sketch of the disease, that there is a state of congestion either co-existent with, or subsequent to, the operation of the exciting cause, it will then be easy to understand why the exhibition of emetics is beneficial. When these induce vomiting, either at the commencement of the affection or when it is fully formed, the effect is, that the sanguineous fluid is more equally diffused throughout the system, and as it is also better oxygenated, the intestines are not only relieved of that quantity of 
blood which impedes their functions, but receive that which is improved in its nature, consequently fitted to promote their ordinary functions.

DXXXIX. We generally find that emetics act as aperients or purgatives; and CULLEN says, that they are useful only when this consequence follows their employment.* But he, and all other writers on the subject, seem to imagine that this effect arises from the medicines passing from the stomach to the intestines, and thus acting directly upon the abdominal viscera. This is certainly a very natural supposition, as we observe that substances which are almost exclusively purgatives, appear to operate in this manner. If we carefully examine the true action of emetics on the general systems of the body, and also reflect on the general effects which follow their exhibition, we shall regard the aperient or purgative consequence as the result of the improved condition of the mucous membrane, from the influence of those causes that relieve the congestion and stimulate the secernent vessels of the part.

DXL. Passive hemorrhages are diseases likely to be benefited by the use of emetics.There is probably no affection to which the human frame is subject that more evidently arises from an altered state of the blood, or

* "As inverting the peristaltic motion, vomits are useful in diarrhca and dysentery; but, independent of that, they are probably more useful as purging the intestines, occasioning a greater flow of liquors into the intestinal canal.'-Lectures on the Materia Medica, by W. Cullen, M. D. \&c. mDcclxxir. 
unequal distribution of $i$, than those included in the order Profluvia. Hemorrhoids, and some of the varieties of menorrhagia, appertaining to the passive kind, have, in many instances been much relieved, if not cured, by the action of vomiting. As I have already stated the manner in which vomiting influences the mode of circulation and quality of the sanguineous fluid, it is unnecessary to dwell longer on the individual diseases belonging to this class. There are several other diseases, not in the least alluded to, in which vomiting has been, or may be, found beneficial, if judiciously prescribed; but, as it is my intention, at the present moment, to select only a few to illustrate the consistency of the views, many disordered states of the body must necessarily be overlooked.

DXLI. We now come to the consideration of the beneficial effects of emetics in croup, diarrhœea, and indigestion, belonging to the second class, improved action of secernent vessels. Emetics, in the former of these diseases, have been employed, in every stage of it, with decided utility. When persevered in at the commencement of the symptoms, the diseased secretion, which ultimately becomes what is called a false membrane, is not allowed to form, because the nature of that which is poured from the mucous follicles is considerably improved; or, if we suppose this membrane to be generated, the same means are efficacious, 
because the quality or character of the secretion is, in the same manner, altered; and, whenever this effect occurs, the membrane becomes less adherent to the internal surface of the trachea, because the diseased matler which connected the two is displaced by what is healthy. Vomiting may sometimes be the cause of removing this, by the agitation which attends its operation; and it will materially facilitate this result, if the mucous membrane to which it is attached has experienced the more extensive and previous influence of those agents that regulate the secretions by improving the qualities or distribution of the blood. DXLII. The mode in which the secretory functions are acted upon by vomiting, is not, on all occasions, easy to explain. The following reasons, which are assigned, may probably be considered to make an approach towards a correct explanation: Inflammation is never absent in croup; indeed, this is the nature of the disease; and, whenever any part of the body becomes the seat of inflammation, it is always accompanied by an increased action, or congested condition of the blood-vessels. The increased action probably depends on an increased quantity of blood which these receive, this also being of a more stimulating description than usual; and the congestion may happen when the vessels are not enabled to circulate the blood transmitted to them, this, on the other hand, being of a less stimulating 
kind. But, whether we regard the vessels in a state of augmented action or of congestion, we may assert, with truth, that these possess a more than ordinary quantity of blood. Either of these states of inflammation being supposed, emetics, when carried to their fullest extent, will distribute the blood more universally, from this being of a more stimulating kind, combined with the muscular exertions of the body ; and, if distant and superficial vessels receive a greater quantity from these causes, those vessels that previously received too much will be deprived of a portion of their superabundance. It is in this manner that rubefacients are supposed to act; and if means which are applied externally, in the vicinity of an affected part, disburthen overloaded vessels, or modify their action, it is certainly allowable to suppose that other means, which operate in a similar manner, but to a much greater degree, ought to be followed by similar results.

DXLIII. The function of digestion is not unusually accelerated by emetics; but as it would occupy a great deal of time to develope the views connected with the origin and influence of its derangement, and, still further, to do ample justice to the principles by which we should be guided in its treatment, I shall refrain from making any observations at present on this important subject. 
DXLIV. Emeties have sometimes been employed, and, with advantage, in different cases of diarrhœa. This disease may be produced by a variety of causes; but it is not improbable that the proximate cause is the same, with slight modifications, in every instance, viz. a morbid increase of action in the secernent vessels of the intestines. When indigestion and crudities of the stomach have been present in this disease, they have generally been regarded as the exciting causes; and, in order to remove them, vomiting has been recommended.* It is impossible to deny that indigestion and crudities of the stomach have very frequently occasioned this condition of the system. If the stomach, from being oppressed either by quantity or improper quality of food, have its ordinary functions disordered, its contents will not be so perfectly concocted as usual, and these, therefore, are likely to irritate the secreting surface of the intestinal canal. But although such causes as these may occasionally exist to produce diarrhœe, yet, I am disposed to think, that the debilitated or deranged state of the system is to be regarded as the principal condition which gives rise to the different disorders of the abdominal viscera. If this view be allowed to be correct, emetics have a more extensive influence upon the animal economy than

* Vide Culden's First Lines of the Practice of Physic, Mccccxcix. 
the mere rejection of crude matters from the stomach. By the operation of such agents, the quality and circulation of the blood undergo material changes, the consequence of which is, that digestion is improved from the better secretion of gastric juice, and at the same time, and from the influence of the same changes, the functions of the rest of the abdominal organs are proportionally benefited.

DXLV. I have already stated, that the present classification cannot be considered correct in all its parts. As our knowledge of the manner in which the exciting causes of diseases affect the system is rendered more exact, we shall be enenabled to speak with more confidence of the general and particular effects of emetics. 'The greater part of the treatment in phthisis, whether local or general, promotes an equable distribution of the blood. The warm bath, exercise, blisters, and stimulants, are evidently to be referred in their operation to the principle of altered circulation. Both the stages of emetic effects have an acknowledged control over the course of the blood; but unless we possess clear and accurate ideas concerning the pathology of consumption, it will be impossible to apply either of these stages or general divisions of emetics to the relief: or cure of the symptoms.

DXLVI. It is, perhaps, of little moment to our mode of treatment, whether we suppose the tubercles or degenerations of the lungs to be the 
consequence of inflammation, or of a scrophulous or peculiar predisposition, if these be fully formed. But when the first symptoms of the affection are slightly developed, the consideration of the nature of those causes to which these are attributable becomes an object of imperative investigation. If, in the first stage of phthisis, a slow inflammatory process be going on, it would seem imprudent to apply means that tend to accelerate this condition; but if disease be gradually destroying the healthy structure of the lungs, without the sanguiferous system being in any degree excited, we may be enabled to employ certain powerful agents that would apparently be improper in the other case. It was the opinion of LAENEc, that this disease was seldom or never radically cured unless the tubercles have passed through the various steps of degeneration to the formation of a cavity, the membranes of which protect the pulmonary tissue from further ravages, and the purulent matter which they secrete escapes through the communication existing between the bronchial ramification and the cavity itself. The obliteration of this communication or cavity constitutes a perfect cure.

DXLVII. If we follow the opinion of $\mathrm{Dr}$ Alison, supported by a variety of well established facts, which seem to show that tubercles are more frequently the effects of inflammation than of any other cause, we shall be influenced accord- 
ingly in our remedial measures. It has been stated, that, in all cases of nausea, the circulation is depressed, because the lungs contain a greater quantity of blood, or blood not oxygenated to its usual extent. The existence of both states is most probable. If inflammation be present, can we diminish the excited action of the morbid part by persevering in a long course of nauseating medicines? This practice has been found useful in pneumonia; but is the analogy between the two affections sufficiently close to allow of the same treatment? If inflammation be the cause of the disordered symptoms in both instances, there is a manifest difference in the extent and severity of the disease, and also in the general consequences.

DXLVIII. I am disposed to regard the follicles of the mucous membrane, or at least those organs appropriated to the secretion of mucus, and which must be supposed to exist throughout the minute bronchial tubes,* as the primary seat of acute or chronic inflammation, producing or concomitant with phthisis. These, by a determination of blood to the lungs, whether from

* "In most mucous membranes are found minute oval or spheroidal bodies, slightly elevated, and presenting an orifice leading to a blind or shut cavity. As they are believed to secrete a fluid analogous to or identical with mucus, they are named mucous glands. In the state of health these bodies are so minute that it is very difficult to recognize them. I have seen them, nevertheless, in the tracheo.bronchial membrane, by the eye and by a lens." - Elements of General and Pathological Anatomy, By David Criarge, M.D.

rf 
cold or any internal derangement, have their natural action disordered,- - the consequence of which is, that an unhealthy secretion is poured out, and probably in greater quantity than is compatible with the well-being of the lungs. This secretion, in the course of its progress, will tend more or less to destroy the permeability of the air-cells in the vicinity of the disease, and will materially contribute to form the various kinds of engorgement particularly described by Andral and Lemnec.

DXLIX. If this pathological view be allowed to be correct, or if chronic inflammation be supposed to constitute the disease, in considering the effects, without in the least ascending to the primary cause, our practice in endeavouring to remove the affection will be directed by the knowledge we possess of the nature of those consequences induced by chronic inflammation. It will teach us, that those means which improve the follicular secretions will the most speedily restore the heallhy function of the lungs. As the effect of nausea is to furnish blood of an inferior quality to the thoracic organs, or rather to engorge than to free them from a partial or general congestion, it is probable that it would not facilitate, if it did not retard, the object that we have in view. Vomiting appears well calculated to benefit the patient. The improved condition of the blood, and its more equal diffusion, are cir- 
cumstances which will tend to stimulate and relieve the vessels, whatever be their natural functions; and if these take upon themselves a healthy action, the incipient tubercles, or mass of pulmonary degeneration, will gradually disappear. That state of the blood which promotes a healthy secretion will also excite the absorbents; and it is probably the combination of those two powers that dissipates the local and general symptoms of consumption. The disease occasionally becomes latent in its effects, or apparently inactive : and whenever the exciting cause is augmented or applied, we have then the usual indications of derangement. The different means employed may retard the farther progress of tuberculous accretion, but may not act to an extent sufficiently great to remove what has already been deposited.

DL. Gentle exercise of every description, and a sea voyage in particular, are recommended in this affection, and have been found more efficient in relieving or curing the symptoms than the exhibition of any internal medicines, or external application. The manner in which these influence the constitution is to be explained only on the assumption that these equalize the circulation, and improve the qualities of the blood. In a sea voyage, vomiting is not at all unfrequent ; and if the patient derives any benefit from being several months upon the F f 2 
sea, I am inclined to believe that no small part of the amendment arises from the repeated occurrence of that action.

I have been led to this opinion, from considering the action of emetics on the circulation of the blood; and the following observations of $\mathrm{Dr}$ REiD, on the cases enumerated by Dr GiLchrist on the benefit of sea voyages to consumptive patients, are corroborative of the principles. "The patients were generally sea-sick, and vomited much bile; and in some the good effects ceased when they grew familiar to the ship's motion, and were no longer sea-sick. He relates the case of a consumptive patient who went to sea three times, the distance ten leagues; each time he was sick, vomited bile, and was cured of his disease. In the last, where the patient was at sea only five or six hours, the effects could not proceed from the air or exercise."*

DLI. The above observations, with respect to the use of emetics, are supposed to apply to the disease at its commencement only, or before the parenchyma of the lungs is very much disorganised. At a later period I should not expect equal success, and, in some instances, should not be surprised if they occasioned injurious consequences.

DLII. Changes in the properties of the blood.That the sanguineous fluid is capable of being

* Wrtsos on Febrile Diseases, p. 544. 
rendered more stimulating than natural, can scarcely be called in question; and, in different parts of this work, I have developed principles which explain the mode in which the blood becomes unfit for the purposes of the system, on account of its deteriorated qualities. Emetics, if they simply cause nausea, will either arrest or retard the flow of blood in all cases of active hemorrhagy. The truth of this is observed in venesection accompanied by faintness. In this instance the stoppage arises from the circulation being generally depressed; the blood has the tendency to move towards the chest and internal viscera in greater quantity than usual: and if the feeling of nausea be continued for a long time the blood becomes less stimulating; so that we have two reasons why emetics, confined to nausea, ought at all times to be prescribed in active hemorrhage. The one is, that the extremities and the superficial vessels receive less of the sanguineous fluid; and the other, that the superabundance, by surcharging the lungs, prevents the ordinary degree of oxygenation, and, on that account, the heart does not propel the blood with its wonted energy.

DLIII. Emetics have been recommended in pneumonic inflammations, by several distinguished continental physicians; by some for the purpose of exciting nausea ; by others of producing vomiting. It is extremely difficult to say whether the 
beneficial effects are to be attributed to the former or to the latter, as it is seldom that we have either of these consequences independent of the other. In 1824 and $1825 \mathrm{I}$ attended to the practice of LANNEC, at the Hospital of La Charité, during which time he treated inflammation of the lungs principally by nauseating doses of tartar emetic, and certainly with success. In the greater number of the cases, slight vomiting was also occasioned.

DLIV. Inflammation of the pulmonary parenchyma differs little in its stages from the same process in every other viscus of the system. The blood-vessels, in the first instance, are more than usually stimulated; but, subsequently, the increased action diminishes, and the vessels are engorged. These two important stages are better illustrated by pneumonia than by any other affection with which I am acquainted. The rale crepitant is the distinguishing characteristic of the incipient symptoms ; and it is not improbable that this peculiar sound arises from the augmented impetus of the blood, its greater quantity, and some slight obstruction to its course. When the disease passes from this condition into that in which some part of the delicate texture of the lungs has become impervious to the air, the circulation is considerably impeded, and the vessels are ultimately congested, giving to the chest a dull sound on percussion. 
DLV. From the nature of these two general indications, I am inclined to think, that the success of vomiting at the commencement of inflammation of the lungs depends much on the existence of peculiar circumstances. If the disease be not very severe, or if a copious perspiration succeeds the exhibition of the emetic, it may be highly beneficial, as it will regulate the distribution of the blood, and remove a portion of the preternatural heat of the body; but if we prescribe these means when the individual is highly plethoric, and the inflammation is intense, without being assisted by a profuse sweat, we shall endanger the life of the patient. At this period. of the symptoms, nausea will be found useful, as it depresses the circulation, and diminishes the stimulus of the blood; but when we have every reason to believe that the pulmonary tissue is congested, the operation of vomiting is to be preferred. The tendency of this action is, as previously stated, to disburthen the lungs, by equalizing the circulation of the blood, and by improving its qualities.

DLVI. The practice of the contincntal physician has not been equally serviceable in England. The varieties of climate, food, and manners, modify very much the success of general principles. Acute inflammatory diseases, in France and Italy, are more easily controlled hy the aid of moderate means than the same in our own country. The substantial and invigo: 
rating regimen of our less genial climate, while it fortifies the constitution against almost every inclemency of the seasons, strengthens also the same against the influence of remedial measures, in the state of disease. There are, however, many instances in which the continental practice with regard to emetics may be adopted with advantage. When the patient is naturally feeble, or debilitated by a tedious plan of treatment, vomiting or nausea may enable us to arrest or retard the progress of the disease.

DLVII. In glancing at the following subject, increased action of the absorbents, there are many difficulties to render the investigation incomplete. Our anatomical knowledge of those vessels which exhale or absorb any of the fluids of the system is necessarily very imperfect, from the minuteness of the objects ; and our acquaintance with the laws by which they are regulated is far from being satisfactory. The labours of Cruickshank, Baillie, Parry, Blackall, and Ayre, have of late years enabled us to make important approximations towards a knowledge of those conditions of the system which predispose to, or occasion an accumulation of serum. The weight of authority inclines to the opinion, that the capillary vessels are in a state of congestion or inflammation in those cases in which dropsy is induced.*

- Vide Chap. XXIII. \$I. Serous Membranes. Craigie's Elements of General and Pathological Anatomy. 
DLVIII. It was the opinion of Dr Cullen, that dropsy was to be attributed either to increased exhalation or diminished absorption; and this view is not at all improbable. We know that different processes are continually going on in the system; for example, that one set of vessels is employed in absorbing, and another in secreting or exhaling. These facts naturally led the mind to conclude, that if we increased the action of the latter, or diminished that of the former, we should have the same results. The reasoning is probably rather mathematically than physiologically correct; it certainly throws little or no light on the pathology of the disease ; and unless the contributions of others in this department had been of a more definite description, our practice in regard to dropsy would not have been improved. As it is now ascertained that congestion or inflammation of the capillary vessels is, in the greater number of instances, the cause of dropsy, we are enabled to bring into operation some of the most powerful means we possess for exciling or depressing the powers of the system according to the character of the symptoms. Depletion, sudorifics, and emetics are the most efficient agents we possess, when judiciously employed, for removing an inordinate exhalation of serum, and for re-establishing a healthy action of the secretory and absorbent organs. If we endeavour to enquire how congestion gives rise to serous accu. 
mulations, we are immediately lost in conjectures or fanciful opinions. It is much better, in the present state of physiologial science, to seize and act on what is certain, and, unless the facts which are possessed allow us to secure a step beyond these, to rest here.

DLXI. Emetics were formerly more frequently used in dropsical affections than at the present day. Fashion, which regulates the most trifling concerns of mankind, has gradually influenced the employment of emetics that regard the powers of life. Every class of medicines is subject to these changes of favour, and from very obvious causes. By experience, or chance, it is discovered that particular substances or means act almost as specifics in this or that kind of diseases. This fact is no sooner proved than the practice is immediately employed generallyby others in the same class of diseases: in some it is found to be beneficial, but in the greater number to be injurious or inefficient. When physiology and pathology have made us better acquainted with the natural or morbid action of the most important functions of the animal economy, we shall then see more clearly than we do, even at present, the absurdity of prescribing remedies, however much extolled, without taking into consideration, the varieties of constitution, the influence of pursuits or habits, the extent or character of the disease, the exciting cause, and the season of the 
year in which the affection occurs. From want of these considerations, medicines of few virtues have occasionally been praised as specifics, and others deserving of this character have been thought to be perfectly harmless.

DLX. Although inflammation or congestion may be the cause, in the greater number of cases, of serous effusions, yet I am disposed to think that these, independently of obstacles to the free circulation of blood, are occasionally the consequence of debility, without this being characterized by either of the above conditions. This opinion is supported by MASON GooD; but the authority of this physician, in regard to physiological or pathological subjects, cannot be considered great. Debility generally induces congestion in those tissues of the body that exhibit a dropsical diathesis.

DLXI. Other substances besides those which are employed as emetics, such as digitalis, squills, and tobacco, are frequently used, and with good effect, in the different kinds of dropsies. These medicines have one common tendency, viz. to produce nausea, and consequently to act powerfully on the sanguiferous system. If the diseases, in these cases, be the result of an inflammatory action, the changes in the distribution and qualities of the blood will tend to destroy this condition; and in proportion as this is accomplished, the capillary vessels will return to their normal state. 
This reasoning is intended to apply principally to inflammation supposed to be accompanied by an increased action in the vessels affected, and not when these are particularly oppressed by congestion. If the latter condition be imagined to be present, vomiting will be beneficial, as the efficacy of its operation is communicated to the circulation and properties of the blood, improving the irregularity of the one and the deficient stimulating agency of the other.

DLXII. If we, for a moment, lay aside the theories of inflammation, and also the consideration of the manner in which different causes act, that predispose to or excite dropsy,-there are two states of the system which co-exist with, or characterize, the different species of this general class of diseases, too important to be overlooked. In the one, we have unequivocal symptoms of inflammation; in the other, the absence of every sign indicative of excited action. The former has been proved to be most successfully treated by those medicines and measures that lessen the inflammatory action, such as by digitalis, squills, and tobacco, and also by the frequent abstraction of blood. The latter, apparently depending on manifest debility, has been prescribed for in almost every possible way. In regard to this the treatment is continually changing, as no particular line of practice has been discovered to authorise the physician to persevere long with 
confidence in any plan, unless attended by almost immediate improvement of symptoms.

DLXIII. From obvious local effects, we are sometimes enabled to estimate the nature and extent of general effects, which are otherwise not to be appreciated, produced by the operation of those causes which are supposed to have occasioned consequences only of a circumscribed character. Vomiting has often been found useful in dropsies of the scrotum and ovarium. The good result has generally been attributed to the sympathy existing between the slomach and these organs of the body. The disease is readily traced to those causes which derange the circulation of blood in the part; and I have already endeavoured to prove, by direct experiment, that vomiting has a more extensive action than the mere agitation of the system, or the awakening of any mysterious sympathy. Its operation tends to improve the properties of the blood by diminishing the proportion existing in the lungs, agreeably to the principles of the first chapter, and it also tends to equalize the distribution of the same throughout the body. These two important conditions are not confined to any single organ of the system, but are common to every viscus and tissue. Is it not, therefore, probable that the accumulated serum disappears from an excited action in the absorbents, derived exclusively from these two conditions of the sanguiferous sys. 
tem? If twenty such local diseases affected the body at the same time, they would all be similarly benefited by these general alterations.

DLXIV. If the serous accumulation be ascribed to diminished absorption, we possess no agents that are so well calculated to excite the diseased function of the absorbents as the whole class of emetics; or if we suppose this to arise from increased secretion or exhalation, the same means will be equally efficient in correcting the disordered function. It may probably be expected that I should explain more particularly how the absorbents and secernents are affected by the exhibition of emetics. In the present state of our physiological knowledge, it is much better to collect and adhere to a few established facts than to attempt to form a complete system by the assemblage of vague opinions and fanciful hypotheses. 


\section{APPENDIX.}



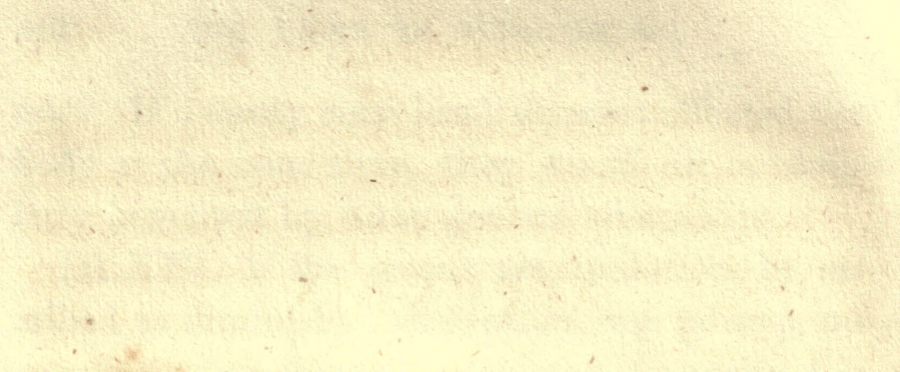

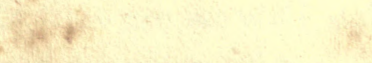

(4)

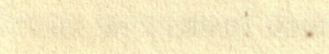

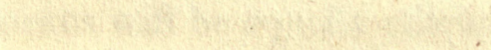

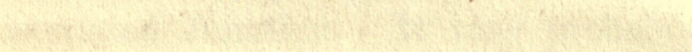

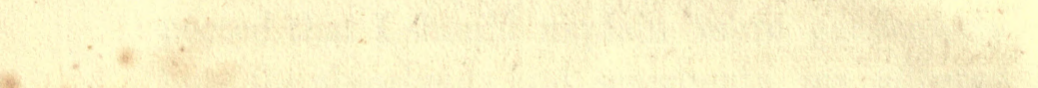

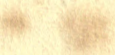

at

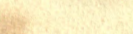

3.

Ion

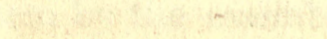

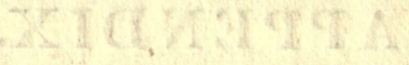

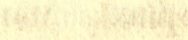

(4)

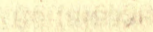

(1)

48.

(4)

is $x^{2}+x^{2}$

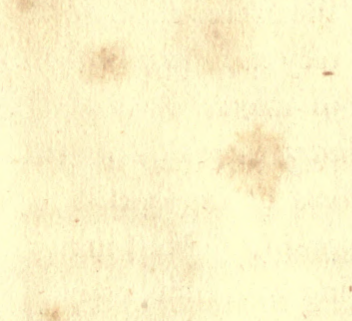

intalis

38

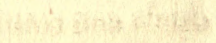

" 


\section{APPEN DIX.}

IN $\$$ XI. XII. page 8 and 9-I have spoken of the influence of inspirations when they preponderate either in number or perfection over the expirations. I have observed that, in such cases, they have the tendency to determine the blood to the internal organs. It may appear to some impossible that either expirations or inspirations can have an ascendancy, since it seems necessary that the quantity of air expired, must, upon the whole, be equal to that which is inspired. But, if we carefully consider the phenomena which accompany either of these respiratory acts, we shall be convinced of the correctness of the above statements. When an individual speaks vehemently, or runs violently, he very soon exhibits shortness of breathing. We perceive that the chest and the shoulders, immediately after the exercise, are raised with great force, arising from the very deep inspirations which the individual is necessitated to make, in order to re-establish in the lungs the ordinary quantity of air, which has been expended in those efforts of the system attendant on running and vehement speaking. Most individuals can count 30 or 40 , commencing with unit, without taking one inspiration. When they arrive at the limit beyond which they cannot pass, they are observed to make a very deep inspiration, which draws in at one time the same quantity of air which had been expelled by 30 or 40 previous 
expirations. The proof of this is, that they can recommence counting with facility. In this illustration we perceive that one inspiration is equal to 30 or 40 expirations, therefore we can say that an inspiration is perfect or complete according to the effects which it produces. The orator can speak for hours without experiencing the want of breath; but this circumstance is evidently to be attributed to the constant pauses, inseparable from temperate or even energetic speaking. Inspirations may preponderate either in number or perfection, from different causes. In one instance, the momentary depressing states of the mind, in the other similar mental conditions, but of a less acute nature, as for example grief, will occasion them. In the latter, the occasional sighs are inspirations, which indicate, in many circumstances, a disordered state of the circulation of blood in the lungs. When inspirations occur in consequence of sudden emotions of the mind, they are excited by impressions which the sensorium has received from some external object; but when they take place in depressing passions of a continuous nature, they are occasionally to be referred to congestion of blood in the lungs, conjoined with the operation of the mental cause. And I may here remark, that, although this preponderance of the one act of respiration over the other is only temporary, and their equilibrium is soon restored, yet the irregularity is often of sufficient continuance to admit of changes taking place fully calculated to account for the morbid results attributed to them in this work.

In $\$$ CCLVI. in speaking of the division of the par vagum in the different experiments performed to elucidate its function, I omitted to state the situation in which the nerve was divided. The division was generally made about three-quarters of an inch below the glottis. 
In page 321, I have stated that the only function immediately relieved by paracentesis abdominis, is that of respiration. Alvine evacuations are sometimes directly promoted by the same means. The relief which is given to the function of respiration is obvious, and seldom fails to succeed the operation; whereas, the intestines are only oc. casionally acted upon in a manifest and beneficial manner by the influence of the same cause.

In \$ CCCCXXII. several theories have been proposed to explain the various symptoms which are exhibited by those who ascend very lofty mountains. SAussure, who had ascended Mont Blanc, and who had observed the different phenomena of the system consequent on such an exertion, as bleeding from the lungs, an unusually turgid and florid appearance of the body, and a distended state of the bloodvessels, imagines that, owing to the great rarefaction of the air, the usual quantity of oxygen is not inspired, and, consequently, the blood does not undergo its usual changes in the lungs. This opinion, as Dr Carson justly remarks, is incorrect, * as the symptoms are not such as characterize the breathing of air in which there is a deficiency of oxygen. There is one observation of SAUSSURE which shows that the explanation which he proposes cannot be considered sound. He says, the difficulty of breathing and faintness almost immediately ceased when the individual rested, and as speedily commenced with the exertions which were made to ascend. This phenomenon clearly proves that the rarefaction of the air is not the only cause; the individual, when he rested, breathed with his ordinary freedom, but when muscular action was employed his respiration became difficult. The condition of the atmosphere, in both instances, is the same.

* An Enquiry into the Causes of the Motion of the Blood, p. 197. 
Dr CARson endeavours to obviate the objections which are made to the theory of SAUSSURE. He attempts to prove that muscular exercise prevents the free return of blood to the heart, and, therefore, when an individual rests in a horizontal position, the blood is transmitted in its usual way to the thorax, and consequently revives the sinking powers of the system. This idea appears plausible, but, if minutely examined, it can scarcely be allowed to be correct. Exercise alone seldom or never produces the peculiar symptoms which are described by SAUSSURE and others as occasioned by an ascent to Mont Blanc, and it has already been observed, that the rarefaction of the air is alone insufficient to give rise to the same symptoms : it is, therefore, manifest, that it is the combination of these two agents, cooperating, as stated in the text, that tends to disturb the whole of the sanguiferous system.

In page 39 I allude to a small machine which I contrived for the purpose of inflating rabbits with warm air. It was constructed as follows :- A common syringe, of about two inches and a half long, was placed in the centre of a small tin box, an inch and a half deep. A brass tube, about the size of a large crowquill, was connected with the syringe, in order to admit the egress of air from, and ingress into it. There was also another brass tube, about one-fourth of the diameter of the preceding, two inches long, soldered to the end of the syringe, which protruded without the box, and which was connected at pleasure to the tube inserted into the trachea of the animal. The box was placed upon four tin legs of two inches and a half long, and was also covered by a lid. The box was filled with water, and afterwards a spirit-lamp was placed underneath, which very soon heated the water in contact with the syringe, and as 


\section{APPENDIX.}

the air, before it passes into the lungs of the rabbit, has to traverse the syringe, it is necessarily warmed by the heated parietes. As it might have been stated that the air, which was sent in, heated the animal to an inordinate extent, I endeavoured to obviate such an objection by ascertaining the temperature of the air during the whole process of inflation. A wooden box, about the size of a walnut, was made of two parts; the bulb of a thermometer was placed within, and when these parts were joined, the neck of the instrument was firmly embraced. The box was afterwards rendered perfectly air-tight by means of sealingwax. At each end of this box was a small brass tube, one of which was connected, at pleasure, to the tube soldered to the end of the syringe, and the other to the tube inserted into the trachea of the animal. As the air passed through the heated syringe into this box before it reached the lungs of the animal, the mercury was raised or depressed according to the temperature of the medium to which it was subjected. In this way it is easy to estimate the temperature of the air which is sent in, and, by keep. ing it many degrees beneath the heat of the system, we are enabled to make the conditions of artificial inflation somewhat similar to those belonging to natural respiration. 'Two wooden screens, separated by about four inches from each other, intervened between the animal and tin box. 'The machine was shown to Dr Milligan, and he approved of the contrivance : It was also exhibited and explained by him to his Physiological Class in the Session of 1829.

THE END. 


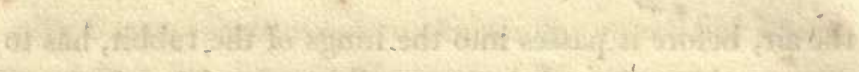

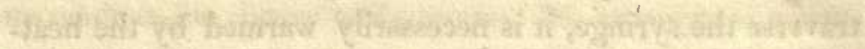

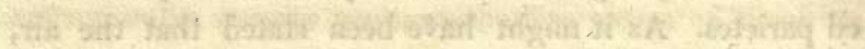

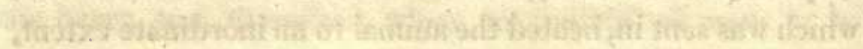

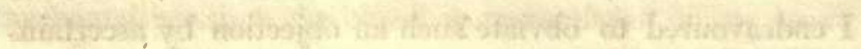

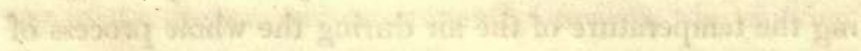

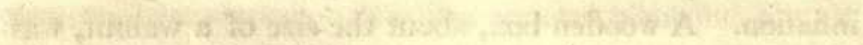

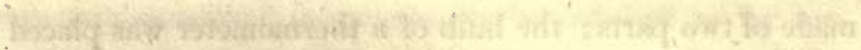

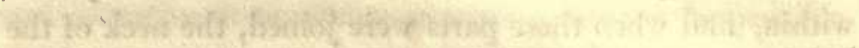

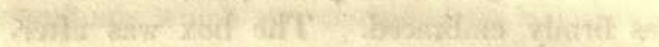

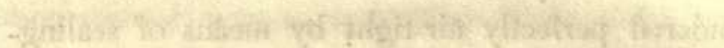

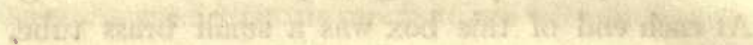

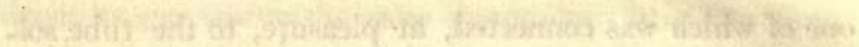

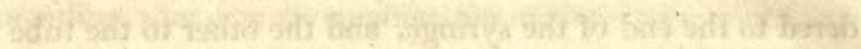

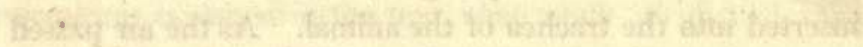

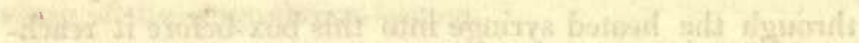

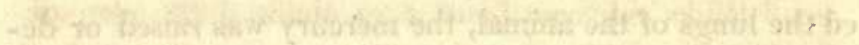

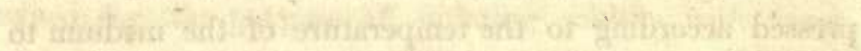

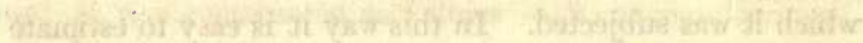

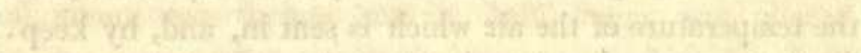

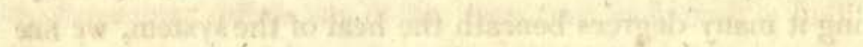

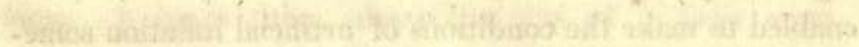

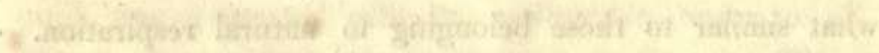

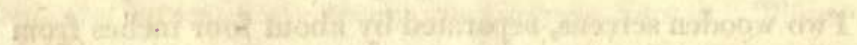

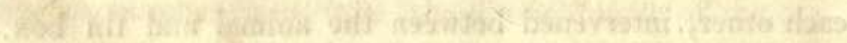

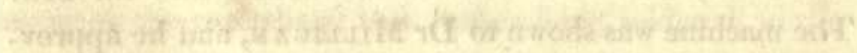

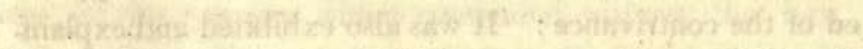

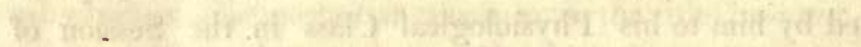

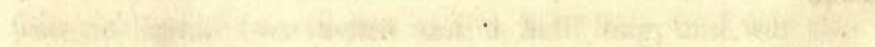




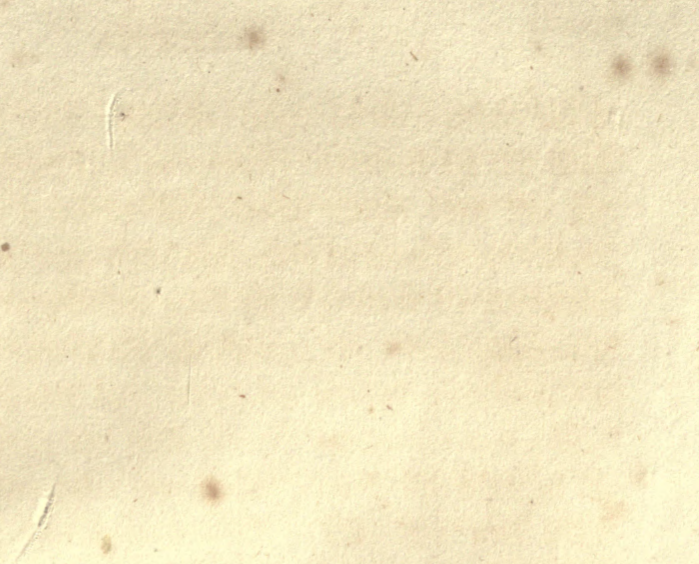






